\title{
Evaluating the Relationships of Phenological and Inter-Annual Landscape Dynamics with Farmland Biodiversity using Multi-Spatial and Multi- Temporal Remote Sensing Data
}

\author{
By \\ Niloofar Alavi \\ A thesis submitted to \\ the Faculty of Graduate and Postdoctoral Affairs \\ in partial fulfillment of the requirements of the degree of
}

Doctor of Philosophy

Department of Geography and Environmental Studies

Carleton University

Ottawa, Ontario

September 2019

(C) Niloofar Alavi, 2019 


\section{Abstract}

Agricultural landscapes are highly variable ecosystems and are home to many species. Farmland spatial heterogeneity and phenological and inter-annual agricultural landscape dynamics has been shown to be related to species diversity. Remote sensing provides data that enable monitoring landscape changes at multiple temporal and spatial scales. The goal of this research was to determine the response of biodiversity to phenological and inter-annual landscape dynamics.

The study area is the predominantly agricultural region of eastern Ontario. Ninety-three sample landscapes were selected prior to this research. Biodiversity data were collected during the summers of 2011 and 2012 within a $1 \times 1 \mathrm{~km}$ area at each landscape. This extent and $3 \times 3$ $\mathrm{km}$ were selected for this research to analyze the impacts of spatial scale on biodiversity response.

Relationships between biodiversity and vegetation phenology were modelled using MODIS NDVI, while relationships between biodiversity and long term inter-annual vegetation changes were modelled using Landsat NDVI and Tasseled Cap components. Random Forest Regression was used to determine relative variable importance over the many biodiversity models produced. The most important variables were identified and subsequently used in step-wise regression to determine model significance, the landscape variables entered, and the direction of their relationship with biodiversity.

Results demonstrated that phenological and inter-annual changes in vegetation dynamics were related to biodiversity. For MODIS, most $3 \times 3 \mathrm{~km}$ models were significant, whereas most $1 \times 1 \mathrm{~km}$ models were not. For Landsat, model performance was not consistently different for the two extents, indicating that model performance can depend on landscape extent when coarse spatial resolution data are used. Plant diversity was lower when the time of onset of greenness 
was later, while beetle diversity was higher when vegetation productivity was higher at the time of onset of greenness. Plant and beetle diversity both were lower when landscape brightness was higher and higher when landscape wetness was higher. The inter-annual temporal variability of Landsat NDVI as well as the percentage of pixels in a landscape with significantly decreasing trends in NDVI demonstrated a negative relationship with plant diversity. This thesis emphasized the importance of spatial and temporal variations in the landscape on biodiversity, especially in highly variable landscapes such as agricultural lands. 


\section{Acknowledgements}

I would like to thank my supervisor, Dr. Doug King for taking me on as a student, mentoring me and supporting me both academically and emotionally both during my whole PhD journey and the thesis process. Thank you for encouraging me during hard times and having faith in me and my way and thank you for all you time and energy and being available to discuss various issues throughout this entire process. I also would like to thank my committee members Dr. Scott Mitchell and Dr. Dennis Duro for providing valuable advice and feedback throughout various stages of this thesis.

I would like to thank the Department of Geography and Environmental Studies at Carleton University for providing me with great financial support and scholarships, teaching assistantship and instructor positions. I am grateful to the Geomatics and Landscape Ecology (GLEL) Lab and to NSERC for providing the funding for this research through a Strategic Project Grant to Drs. Fahrig, King, Lindsay and Mitchell and through a Discovery Grant to Dr. King.

I would like to express my sincere gratitude to my colleagues at Agriculture and Agrifood Canada, Science and Technology Branch, Earth Observation (STB-EO) team, especially Drs. Andrew Davidson, Catherine Champagne and Laura Dingle Robertson for providing me with the MODIS data and relevant technical tools that I needed for this research. I also would like to express my deep appreciation to my colleagues at Environment and Climate Change Canada, Landscape Science and Technology Division (LSTD), National Wildlife Research Centre, especially Dr. Darren Pouliot, for providing me with technical tools and valuable comments on the temporal analysis of MODIS and Landsat data of this thesis.

Finally, I would like to thank my family for their encouragement and endless support of all kinds, my mother, Zari, and my late father, Mohammad who taught me the value of science 
and higher education, my siblings, Saman, Sepideh and Ali who lead me by example, and my closest friends and besties Hoda, Maryam, Neagr and Katty for never giving up believing in me and always being by my side despite the far distance between us. 


\section{Table of Contents}

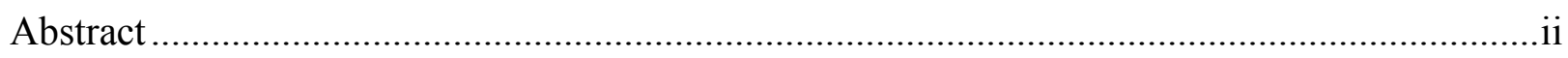

Acknowledgements ........................................................................................................

Table of Contents

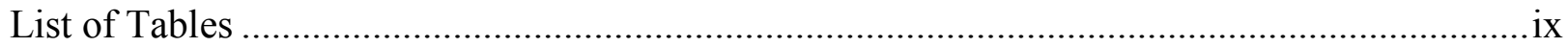

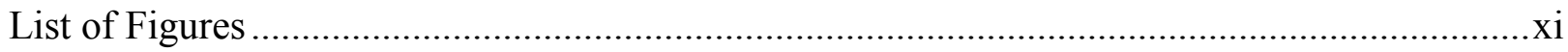

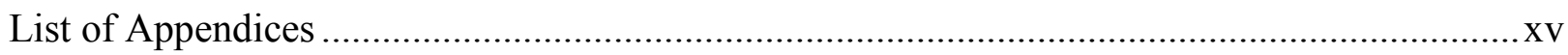

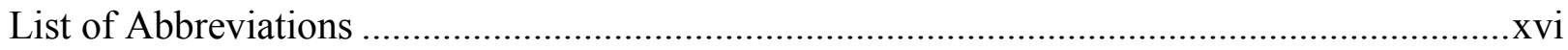

Chapter 1: Introduction ........................................................................................... 1

1.1. Biodiversity and its importance in agro-ecosystems ....................................................

1.2. Biodiversity and landscape heterogeneity in agro-ecosystems........................................ 1

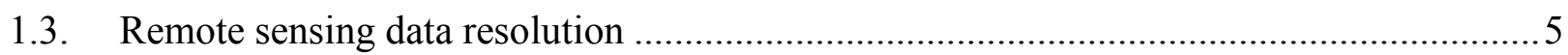

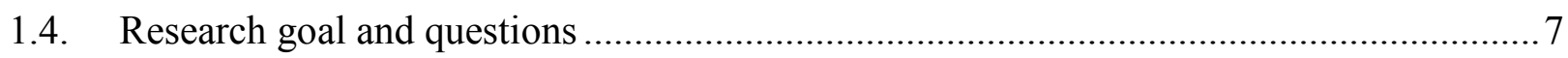

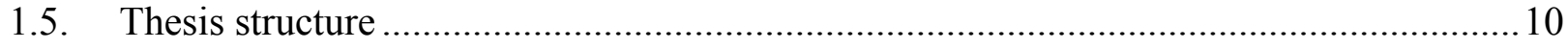

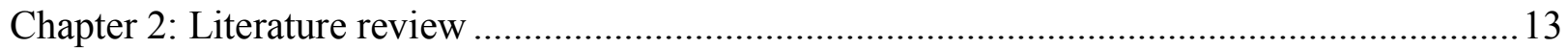

2.1. Biodiversity: concepts and relations with agricultural landscape heterogeneity ..............13

2.2. Spatial and temporal scale of study in biodiversity modeling ........................................23

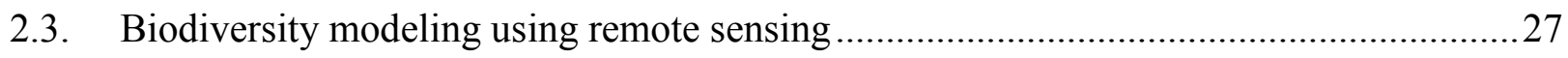

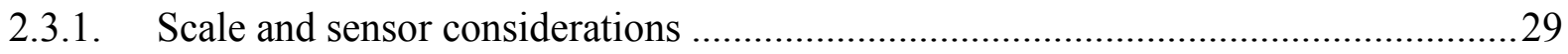

2.3.1.1. MODIS and Landsat time series for analysis of vegetation dynamics ...............32

2.3.1.2. Remote sensing approaches in biodiversity modelling .......................................39

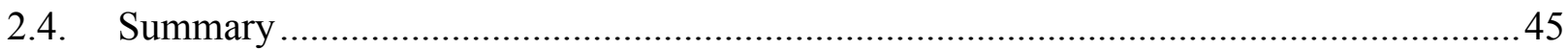

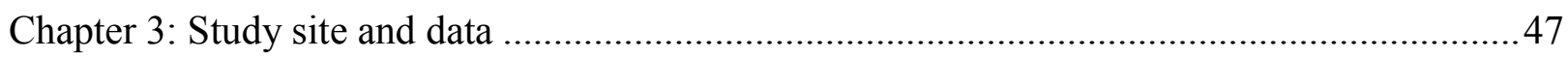

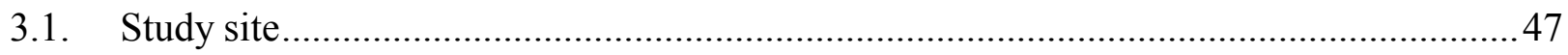

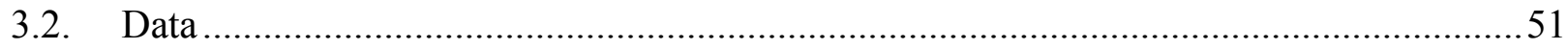

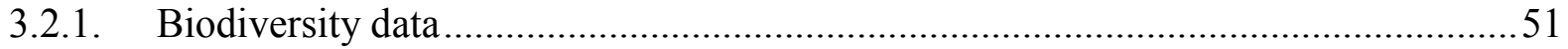

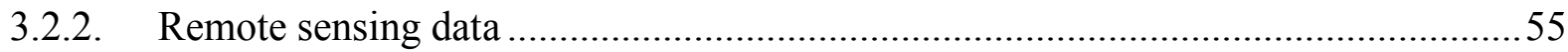

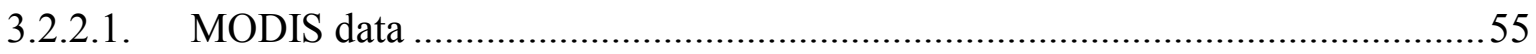

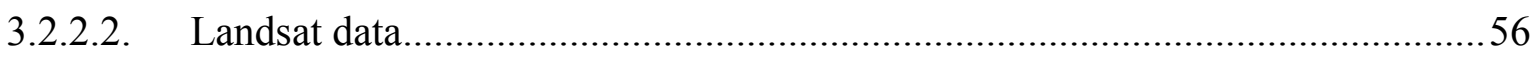

Chapter 4: Modeling the relationship between vegetation phenology and local biodiversity of agricultural lands in Eastern Ontario using MODIS time-series data..........................................57

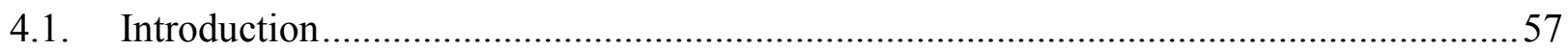

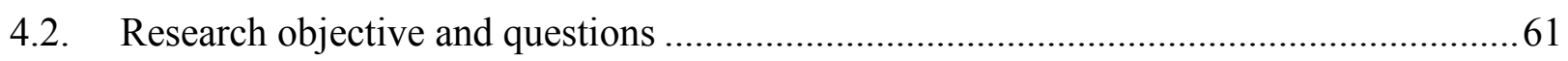

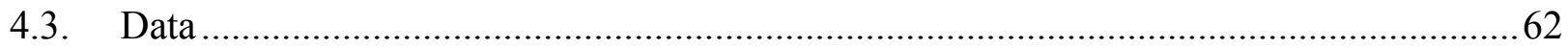




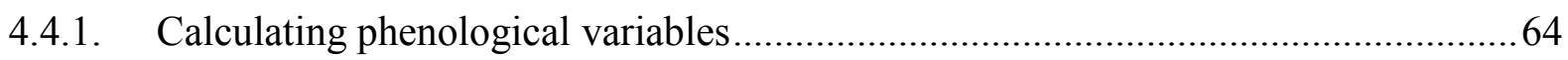

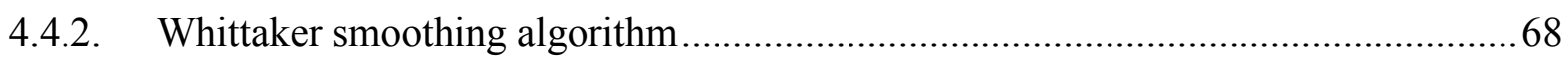

4.4.3. Extracting phenological variables for sample landscapes .........................................69

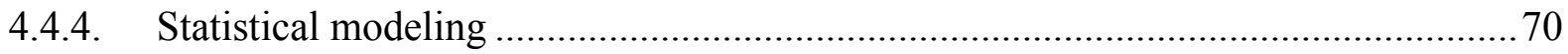

4.4.4.1. Exploratory modeling for selecting response variables ....................................70

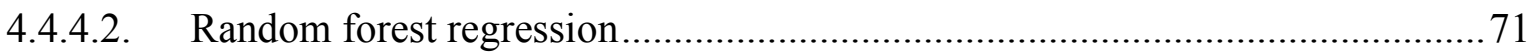

4.4.4.3. Step-wise multiple linear regression ...........................................................

4.4.4.4. Alternative statistical methods …………………......................................... 76

4.4.4.5. Evaluating potential lag effect....................................................................... 78

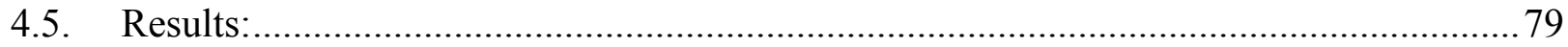

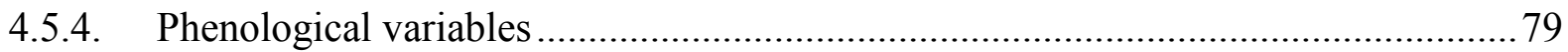

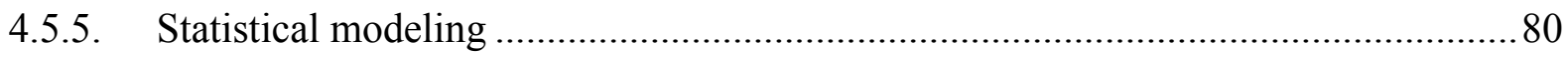

4.5.2.1. Exploratory modeling for selecting response variables ..................................... 80

4.5.2.2 Random forest biodiversity modelling...................................................................... 84

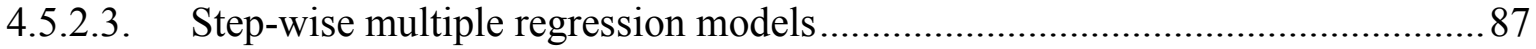

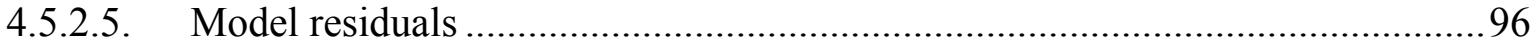

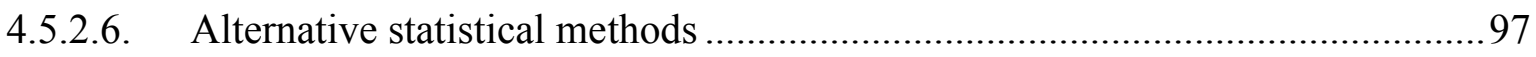

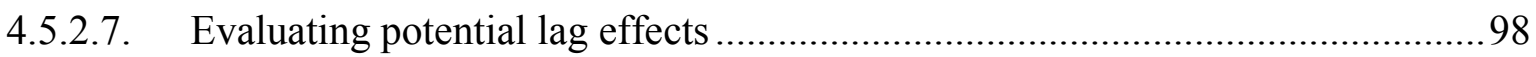

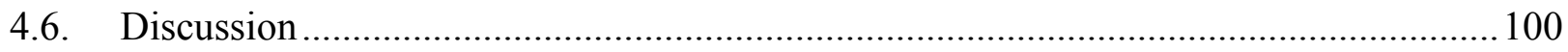

4.6.1. Interpretation of main findings and relations to previous work ............................100

4.6.2. Limitations and recommendations for future research ........................................... 104

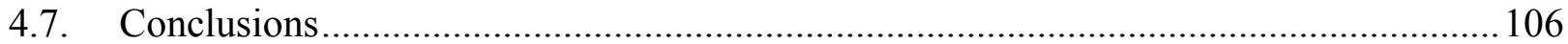

Chapter 5: Modeling the relationship between long-term landscape dynamics and local biodiversity of agricultural lands in Eastern Ontario using Landsat time-series ........................107

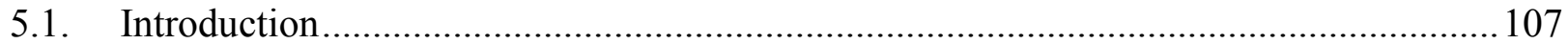

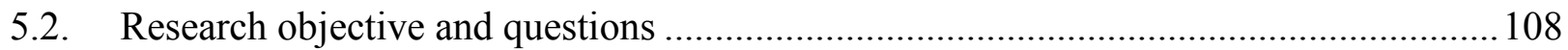

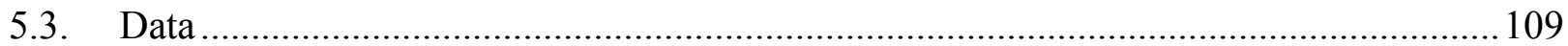

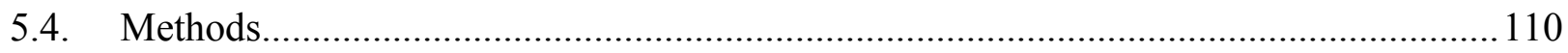

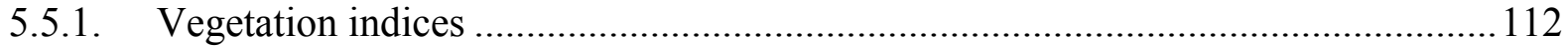

5.5.2. Tasseled Cap components ..................................................................................... 113

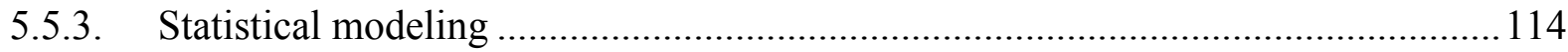

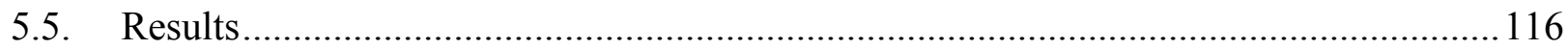

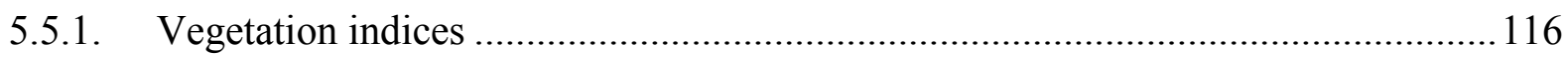


5.5.2. TCT components

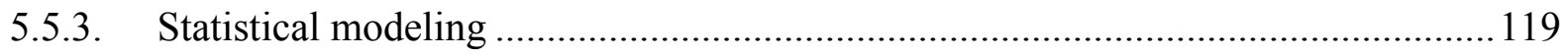

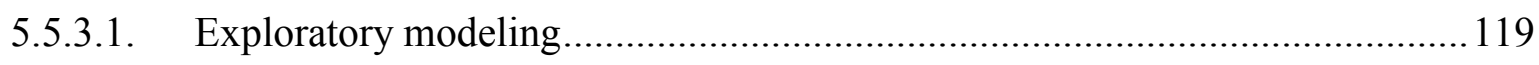

5.5.3.2. Random forest biodiversity modelling .................................................... 122

5.5.3.3. Stepwise multiple regression models ..................................................... 125

5.5.3.4. Significant variables in SWR models and the magnitude and direction of effects 126

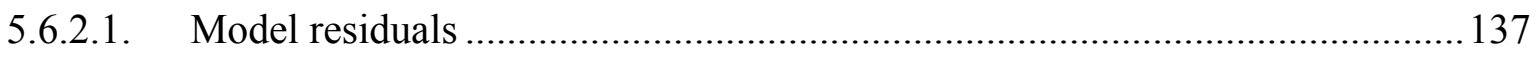

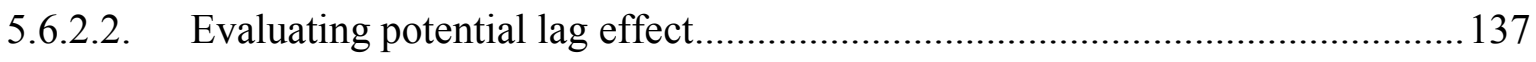

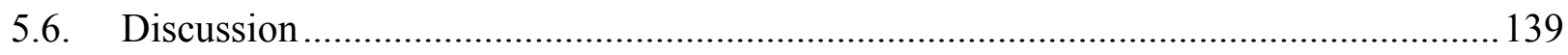

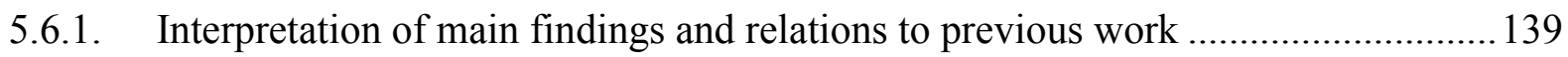

5.6.2. Limitations and recommendations for future research .................................... 144

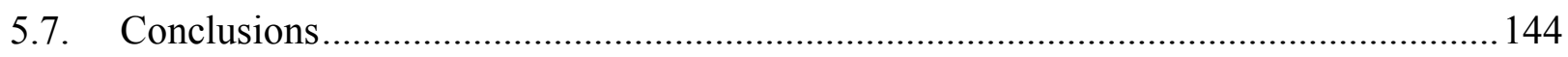

Chapter 6: MODIS and Landsat inter-annual landscape trends and their relationships with

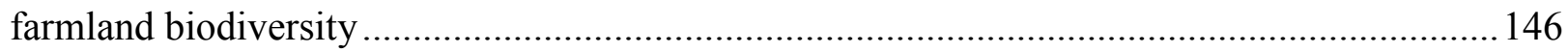

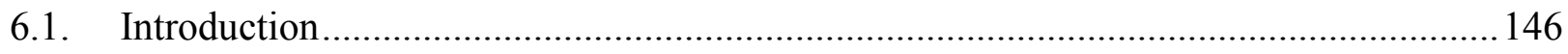

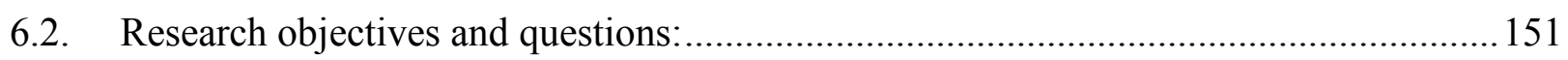

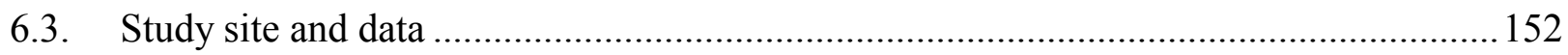

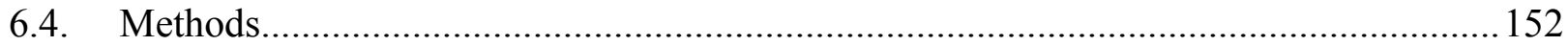

6.4.1. Exploratory trend analysis using time series of MODIS phenology metrics and

Landsat mid-summer NDVI and TCT components: Regional scale ................................. 154

6.4.2. Comparison of MODIS and Landsat mid-summer NDVI trends: Landscape scale 154

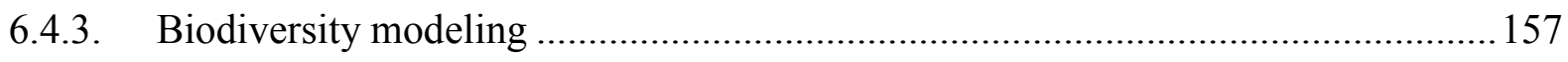

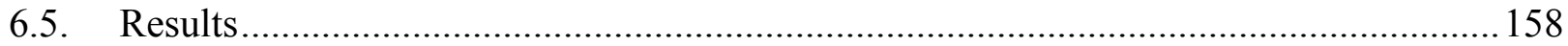

6.5.1. Exploratory trend analysis using time series of MODIS phenology metrics and

Landsat mid-summer NDVI and TCT components: Regional scale ................................ 158

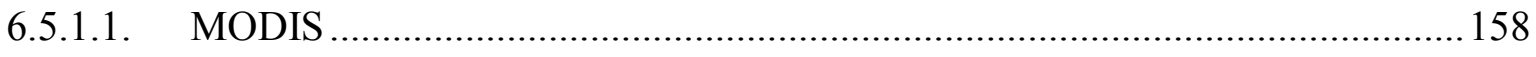

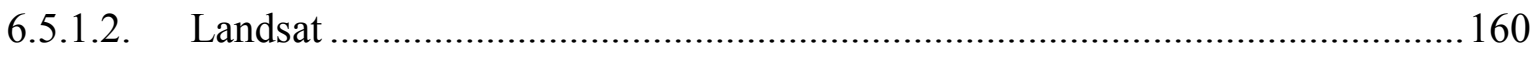

6.5.2. Comparison of MODIS and Landsat mid-summer NDVI trends: Landscape scale 164

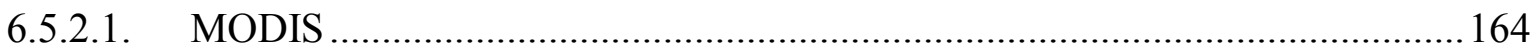

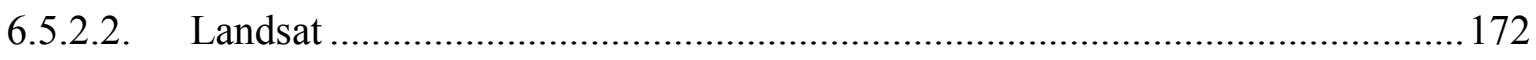

6.5.2.3. Comparing MODIS and Landsat NDVI trend analyses .............................. 185

6.5.3. Modelling biodiversity and inter-annual landscape dynamics ............................ 188

6.5.3.1. Relationships between biodiversity and the proportion of landscape pixels with

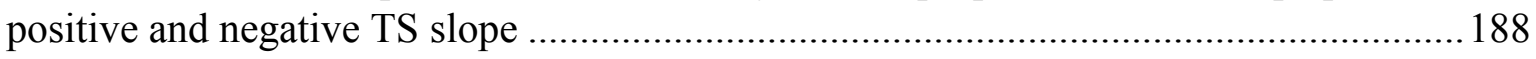


6.5.3.2. Relationships between biodiversity and landscape vegetation dynamics ........ 190

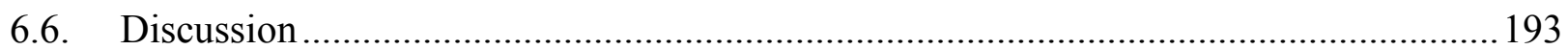

6.6.1. Interpretation of main findings and relations to previous work .......................... 193

6.6.2. Limitations and recommendations for future research .................................... 197

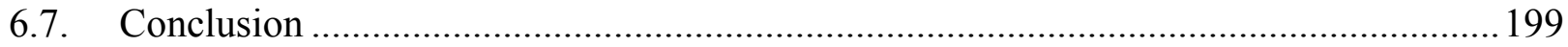

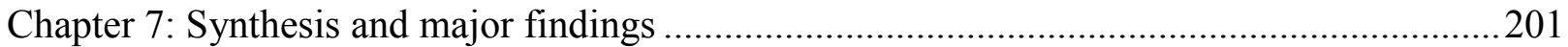

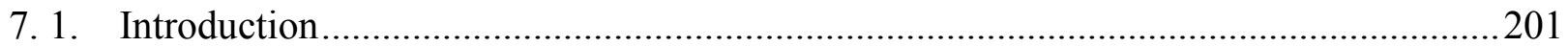

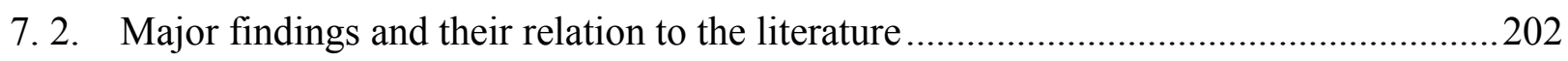

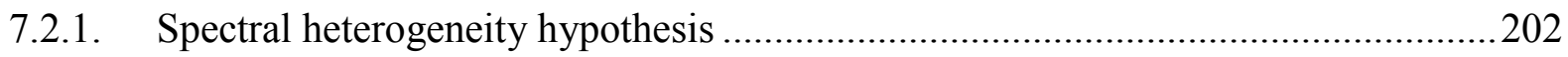

7.2.2. Effects of landscape temporal dynamics of biodiversity ....................................203

7.2.3. Taxa sensitivity to temporal landscape dynamics and possible causes ..................205

7.2.4. Comparing to previous research .......................................................................... 207

7. 3. Alternative approaches and recommendations for further research in biodiversity

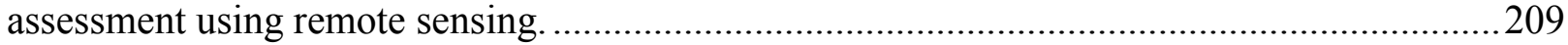

7. 4. Implications of this research for policy and monitoring of biodiversity .....................212

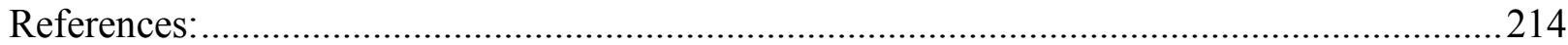

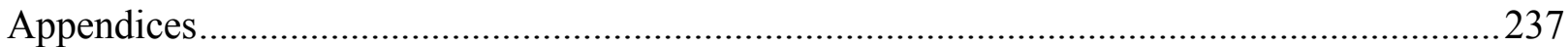




\section{List of Tables}

Table 4. 1. List of models with best explanatory variable of each year to test time lagged response of species to phenological changes .78

Table 4.2. Pearson $r$ values for correlations between the percentage agriculture land cover in each sample landscape at $1 \times 1 \mathrm{~km}$ and $3 \times 3 \mathrm{~km}$ extent, and each biodiversity variable for the four selected taxa. Significant $(\mathrm{p} \leq 0.05)$ relationships are shown in bold. .82 Table 4.3. R values between the $\% \mathrm{Ag}$ and $3 \times 3 \mathrm{~km}$ explanatory variables extracted from 2011 MODIS data. Significant $r$ values $(\mathrm{p}<0.05)$ are in bold. .83 Table 4.4. Error measures from random forest regressions of 2011-2012 gamma diversity summarized by taxon for models derived using MODIS phenological indices for 2011 and 2012. The percent variance ( $\%$ Var) and mean of squared residuals (MSR) were calculated from random subsets of one third of the reference samples in each tree. RFR runs were implemented for $1 \times 1$ $\mathrm{km}$ to $3 \times 3 \mathrm{~km}$ landscapes independently. Range of gamma diversity for each taxon is in brackets beside the taxon name. 86

Table 4.5. The nine most important variables selected by random forest regression models for each taxon and the sum of the frequency of their occurrence in models over all 13 years (20002012) and the percentage of occurance over all iterations (1300). Results are presented for $1 \times 1$ $\mathrm{km}$ and 3 x $3 \mathrm{~km}$ landscapes.

Table 4.6. MODIS phenological variables entered into SWR models and the number of years (out of 13) each variable was entered into a model for each taxon and $3 \times 3 \mathrm{~km}$ landscapes

Table 5.1. Year and date of the selected Landsat 5 TM data 110

Table 5.2. TM bands coefficients for each TCT component

Table 5. 3. List of models with best explanatory variable of each year to test time lagged response of species to phenological changes

Table 5.4. Pearson correlation between the landscape mean of the four vegetation indices derived from 2011 Landsat data for $3 \times 3 \mathrm{~km}$ landscapes. $\mathrm{P} \leq 0.05$ for all correlations.

Table 5.5. R-values between $\% \mathrm{Ag}$ and $3 \times 3 \mathrm{~km}$ explanatory variables in 2011 . Significant relationships $(\mathrm{p} \leq 0.05)$ are shown in bold.

Table 5.6. Pearson correlation matrix for all explanatory variables in the $3 \times 3 \mathrm{~km}$ landscape models for 2011. Correlations significant at $\mathrm{p} \leq 0.05$ are in bold.

Table 5.7. Error measures from the random forest regressions summarized by taxon for 2011 . The percent variance (\% Var) and mean of squared residuals (MSR) were calculated on one third of the samples in each tree created in the RFR runs for $1 \times 1 \mathrm{~km}$ to $3 \times 3 \mathrm{~km}$ models independently.

Table 5.8. Relative importance of explanatory variables as a ranked list of their number of occurrences in RFR models for $1 \times 1 \mathrm{k} \mathrm{m}$ and $3 \times 3 \mathrm{~km}$ landscape extents.

125

Table 5.9. The number of years out of 16 for which each remote sensing variable entered the SWR models for each taxon.

Table 6.1. The average of climate data for three Environment and Climate Change Canada

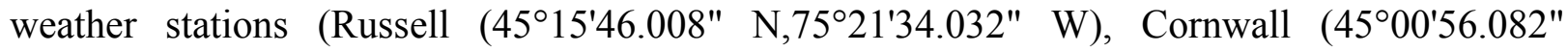

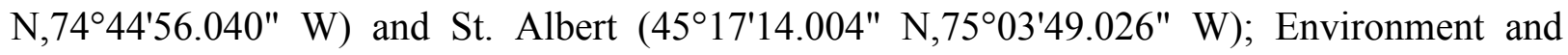
Climate Change Canada, 2018). 
Table 6.2. The proportion of pixels in landscapes with significant positive and negative trends by landscape heterogeneity category.....

Table 6.3. Proportion of positively trended pixels in each landscape heterogeneity grouping. 180 Table 6.4. Proportion of negatively trended pixels in each landscape heterogeneity grouping. 180 Table 6.5. Cross-tabulation of numbers of pixels identified as having significant trends or no trend in MODIS and Landsat mid-summer NDVI time series. ............................................... 186

Table 6.6. Correlation matrix for the temporal explanatory variables.................................. 193 


\section{List of Figures}

Figure 3.1. The sample landscapes. Different square sizes indicate the 1, 2 and $3 \mathrm{~km}$ extent of each landscape, and colours represent the different categories of landscape compositional and configurational heterogeneity (Adapted from Pasher et al., 2013).

Figure 3.2. Example $3 \times 3 \mathrm{~km}$ landscapes representing a) high compositional-high configurational heterogeneity (H_H_18), b) low compositional-low configurational heterogeneity (L_L_204), c) high compositional-low configurational heterogeneity (H_L_35), and d) low compositional-high configurational heterogeneity (L_H_78).

Figure 3.3. Examples of survey transects used for plant as well as butterfly, syrphid, bee spider and beetle surveys. Bee pan traps appeared in the left photo. Photo courtesy of Jude Girard, 2012.

Figure 3.4. Capturing butterflies for identification by a butterfly net of $38 \mathrm{~cm}$ diameter. Photo courtesy of Jude Girard, 2012.

Figure 3.5. Ground beetle and spider traps. Left: the pitfall trap; right: the trap cover. Photo

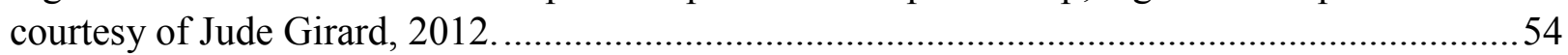

Figure 3.6. Bee pan traps. Photo courtesy of Jude Girard, 2012.

Figure 4. 1. Flow chart outlining various processing and analytical steps of this study. This includes the extraction of phenological variables from MODIS weekly Max NDVI and selection of biodiversity response variables and taxa, as well as the statistical modeling to assess the most important image variables and the direction and magnitude of their relationships with biodiversity.

Figure 4. 2. The Gaussian fit function calculates SOS as $40 \%$ of the difference between first NDVI observation and maximum NDVI and EOS as $40 \%$ of the difference between maximum NDVI and the last NDVI observation.

Figure 4. 3. Raster representing SOS DOY for year 2011. The sample landscapes are shown as black squares. .79

Figure 4.4. Raster representing SOS NDVI for year 2011. The sample landscapes are shown as black squares. .80

Figure 4.5. Comparing the relationship of alpha, beta and gamma diversity of beetles with Mean SEAS INT in 2011. An example of poorer relationships found for alpha and stronger relationships for beta and gamma diversity.

Figure 4. 6. Absolute r-value for all models. Orange represents $1 \times 1 \mathrm{~km}$ models and blue represents $3 \times 3 \mathrm{~km}$ models. Significant variables in step-wise models and the strength and direction of their relationships with gamma diversity. Dotted lines represent $r$ when $p=0.05$ for each corresponding landscape extent.

Figure 4.7. R-values and variables entered into SWR models of gamma diversity in each corresponding year for plants and 3 x $3 \mathrm{~km}$ landscapes. Error bars present 95\% confidence intervals. 92

Figure 4.8. R-values and variables entered into SWR models of gamma diversity in each corresponding year for beetles and $3 \times 3 \mathrm{~km}$ landscapes. Error bars present 95\% confidence intervals. 
Figure 4. 9. R-values and variables entered into SWR models of gamma diversity in each corresponding year for birds and $3 \times 3 \mathrm{~km}$ landscapes. Error bars present $95 \%$ confidence intervals. .95

Figure 4. 10. R-values and variables entered into SWR models of gamma diversity in each corresponding year for butterflies and 3 × $3 \mathrm{~km}$ landscapes. Error bars present $95 \%$ confidence intervals. .96

Figure 4. 11. Example residual plot of beetle gamma diversity model for Mean EOS NDVI in 2012 .

Figure 4. 12. Pearson $r$ for lag effect models of NDVI CV against plant gamma diversity. A jump in the $r$ value was evidenced in M6 where NDVI CV of 2007 was added to the model. 100

Figure 5. 1. Flow chart outlining various steps used in this chapter. This includes the extraction of TCT and vegetation indices from Landsat 5 archive and selection of response variables, as well as the statistical modeling to assess the most important variables and the direction and magnitude of relations with gamma diversity.

Figure 5. 2. Example NDVI map of the study area for 2011 Landsat data. Land surface NDVI values were $>0$, while water pixels were $\leq 0$.

Figure 5.3. Comparing the sensitivity of NDVI and TDVI (derived from 2011 Landsat data as an example) in capturing the within-field and between-field variability in vegetation density. Red ellipses delineate example areas of higher density vegetation at which TDVI tends to saturate.

Figure 5.4. TCB, TCG and TCW images of the study region, 1985

Figure 5.5. Comparing the spatial association of 1985 Landsat TCB (shown in yellow), TCG (shown in green) and TCW (shown in blue) in a) high density vegetation croplands, b) wooded areas, and c) buildings.

Figure 5.6. Comparing the relationship of alpha, beta and gamma diversity of beetles with Mean TCB derived from 1994 Landsat data for 3 x $3 \mathrm{~km}$ landscapes. 121

Figure 5.7. Absolute $\mathrm{r}$ value for all models; Orange represents $1 \times 1 \mathrm{~km}$ models and blue represents $3 \times 3 \mathrm{~km}$ models. Dashed lines represent significant $r$ when $p=0.05$ for each corresponding landscape extent. Solid lines represent noticeable but non-significant trends of change in the strength of models from 1985 to 2011. 126 Figure 5.8. R-values and their $95 \%$ confidence intervals for beetle gamma diversity in $1 \times 1 \mathrm{~km}$ (a) and $3 \times 3 \mathrm{~km}$ (b) models. The explanatory variables shown are those that produced significant models in one or more years.

Figure 5.9. R-values and their $95 \%$ confidence intervals for plant gamma diversity at $1 \times 1 \mathrm{~km}$ (a) and $3 \times 3 \mathrm{~km}$ (b) models. The explanatory variables shown are those that entered SWR regression and produced significant models in one or more years.

Figure 5.10. R-values and their 95\% confidence intervals for bird gamma diversity at $1 \times 1 \mathrm{~km}$ (a) and $3 \times 3 \mathrm{~km}$ (b) models. The explanatory variables shown are those that entered SWR regression and produced significant models in one or more years.

134 Figure 5.11. $\mathrm{R}$ values and their $95 \%$ confidence intervals for butterfly gamma diversity at $1 \times 1$ $\mathrm{km}$ (a) and $3 \times 3 \mathrm{~km} \mathrm{(b)} \mathrm{models.} \mathrm{The} \mathrm{explanatory} \mathrm{variables} \mathrm{shown} \mathrm{are} \mathrm{those} \mathrm{that} \mathrm{entered} \mathrm{SWR}$ regression and produced significant models in one or more years.

Figure 5.12. Example residual plot for the $3 \times 3 \mathrm{~km}$ beetle model of 2011 with Mean NDVI as the entered explanatory variable. 
Figure 5. 13. Pearson $r$ for lag effect models of Mean TCB against plant gamma diversity. A jump in the $\mathrm{r}$ value was evident in M11 where the Mean TCB of 1997 was added to the model. ..... 138

Figure 6. 1. Flow chart outlining various steps used in this chapter. This includes linear trend analysis of MODIS and Landsat NDVI time series and modeling the temporal changes against gamma diversity for the selected taxa.

Figure 6.2. Inter-annual variability and linear trends of MODIS-derived phenological variables averaged over all $3 \times 3 \mathrm{~km}$ sample landscapes $(\mathrm{N}=93$, bars show \pm 2 standard deviations)... 163 Figure 6.3. The relationship between Mean SOS DOY and the first DOY with no reported snow on the ground. 163 Figure 6.4. Inter -annual variability and linear trends of Landsat-derived mid-summer variables averaged over all $3 \times 3 \mathrm{~km}$ sample landscapes $(\mathrm{N}=93$, bars show \pm 2 standard deviations)... 165 Figure 6.5. Examples of $\mathrm{H}_{-} \mathrm{H}, \mathrm{L} \_\mathrm{L}, \mathrm{H} \mathrm{H}_{-} \mathrm{L}$ and $\mathrm{L} \_\mathrm{H}$ sample landscapes with no significant MODIS mid-summer NDVI trend (spatial average of NDVI across each sample landscape, $p>0.05$ ). 166 Figure 6.6. Examples of $\mathrm{H}_{-} \mathrm{H}, \mathrm{L}_{-} \mathrm{L}, \mathrm{H} \mathrm{H}_{-} \mathrm{L}$ and $\mathrm{L}_{-} \mathrm{H}$ sample landscapes with a significant positive MODIS mid-summer NDVI trend (spatial average of NDVI across each sample landscape, $p \leq$ $0.05)$. 166

Figure 6.7. Comparing MODIS NDVI average of landscape TS trend slope (irrespective of significance level) for 2000-2011 in: a) H_H, b) H_L and L_H and c) L_L sample landscapes.

Figure 6.8. Theil-Sen slope for 2000-2011 derived from average July MODIS NDVI. 170 Figure 6.9. Mann-Kendall significance of TS slope for 2000-2011 derived from weekly MODIS NDVI. Red and green represent significant negative and positive trends, respectively $(p \leq 0.05)$. 171

Figure 6.10. Examples of significant positive (green) and negative (red) inter-annual mid-summer MODIS NDVI trends and their association with land cover. The $3 \times 3 \mathrm{~km}$ landscape bounds are shown as yellow squares.

Figure 6.11. Examples of $\mathrm{H}_{-} \mathrm{H}, \mathrm{L} \_\mathrm{L}, \mathrm{H} \_\mathrm{L}$ and $\mathrm{L} \_\mathrm{H}$ sample landscapes with no significant Landsat mid-summer NDVI trend (spatial average of NDVI across each sample landscape, $\mathrm{p}>0.05$ ). 173 Figure 6.12. Examples of $\mathrm{H}_{-} \mathrm{H}, \mathrm{L} \_\mathrm{L}, \mathrm{H} \_\mathrm{L}$ and $\mathrm{L}_{-} \mathrm{H}$ sample landscapes with significant positive Landsat mid-summer NDVI trend (spatial average of NDVI across each sample landscape, $p \leq$ $0.05)$. 174

Figure 6.13. Example of temporal break in the trends of average mid-summer Landsat NDVI (for landscape H_L_40). 174 Figure 6.14. Comparing mid-summer Landsat NDVI TS trend slope for 1985-2000 and 20002011 in: a) H_H, b) H_L and L_H and c) L_L sample landscapes......................................... 176 Figure 6.15. Theil-Sen slope for 1985-2011 derived from mid-summer Landsat NDVI. ..........177 Figure 6.16. Mann-Kendall significance of slope for 1985-2011 derived from mid-summer Landsat NDVI. Red represents a significant negative trend; green represents a significant positive trend $(\mathrm{p} \leq 0.05)$. 178 Figure 6.17. Proportion of pixels with positive mid-summer Landsat NDVI trends in all sample landscapes during 1985-2000 compared to 2000-2011. 179 Figure 6.18. Direction of significant $(\mathrm{p} \leq 0.05)$ Mann-Kendall) trend slopes for mid-summer Landsat NDVI: 1985-2000 (bottom) and 2000-2011 (top) 182 
Figure 6.19. Comparing mid-summer Landsat NDVI trends for 1985-2000 and 2000-2011 in example landscapes. (top) Theil-Sen slope for all pixels. Red represents negative slope and green represents positive slope, and (bottom) Mann-Kendall significance. Red represents a significant negative trend and green represents a significant positive trend $(\mathrm{P} \leq 0.05)$.

Figure 6.20. Examples of CMK significant negative trends in mid-summer Landsat NDVI for 2000-2011 associated with natural and semi-natural patches (yellow arrows). 184

Figure 6.21. Examples of CMK significant negative trends in mid-summer Landsat NDVI for 2000-2011 associated with individual fields (yellow circles).....

Figure 6.22. Examples of CMK significant negative trends in mid-summer Landsat NDVI for 2000-2011 associated with individual field edges (yellow circles). 184 Figure 6.23. Comparing Landsat (top) and MODIS (bottom) spatial patterns of significant trends in mid-summer NDVI. Red = negative and green = positive trends. Ellipses with the same colours show the same location on both maps with spatially overlapping significant trends. Arrows show instances of significant positive or negative trends identified by only one dataset. 186 Figure 6. 24. Comparing Landsat (top) and MODIS (bottom) patterns of CMK significant trends in mid-summer NDVI and examples of the spatial distribution of significant negative and positive pixels in part of the study area. Red = negative and green $=$ positive trends. Ellipses/circles with the same colours show locations with spatially overlapping significant trends. Arrows show instances of significant positive or negative trends identified by only one dataset.

Figure 6.25. The relationships between plant gamma diversity and the percentage of pixels in each landscape with negative (a) and positive (b) TS slopes as well as the percentage of pixels with no trend (TS slope $=0.00)(\mathrm{c})$ for given time periods. TS slopes were derived from Landsat mid-summer NDVI time series. 188 Figure 6.26. The relation between beetle gamma diversity and the percentage of pixels with no TS slope $($ TS slope $=0.00)$ in the period 2000-2011. TS slopes were derived from Landsat NDVI time series.

Figure 6.27. The relation between plant gamma diversity and the percentage of CMK significant positive Landsat inter-annual NDVI pixels and no trends in the period 2000-2011. Figure 6.28. The relationship between Landsat-derived temporal CV of mean landscape NDVI for 1985-2011 and plant and beetle gamma diversity of 2011-2012.

Figure 6.29. The relationship between Landsat-derived temporal CV of mean landscape NDVI for 1985-2000 and plant and beetle gamma diversity of 2011-2012. 192 Figure 6.30. The relationship between Landsat-derived temporal CV of Mean landscape NDVI for 2000-2011 and plant and beetle gamma diversity of 2011-2012. 192 


\section{List of Appendices}

Appendix I. List of species identified in the 2011 and 2012 field surveys as presented in Fahrig

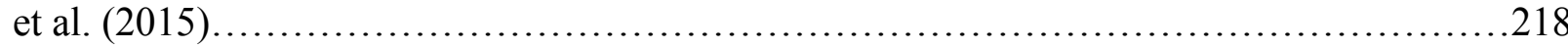

Appendix II. Remote sensing data pre-processing .................................226

Appendix III. Correlation matrices of MODIS 2011 and 2012 explanatory variables for $3 \times 3$ km sample

landscapes. .244

Appendix IV: RFR mean square residuals and percent variance accounted for: MODIS and Landsat data, $1 \times 1 \mathrm{~km}$ and $3 \times 3 \mathrm{~km}$ sample landscapes for each taxon and each year

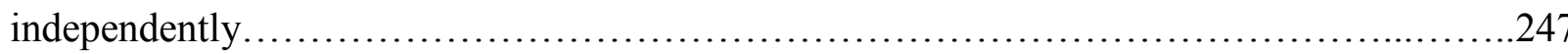

Appendix V. Example of the output of stepwise regression models.....................252 


\section{List of Abbreviations}

AAFC: Agriculture and Agrifood Canada

AVHRR: Advanced Very High Resolution Radiometer

BBS: Breeding Birds Survey

CALMS: Canadian Ag-Land Monitoring System

COSEWIC: Committee on the Status of Endangered Wildlife in Canada

CMK: Contextual Mann-Kendall

$\mathrm{CV}$ : Coefficient of Variation

DOY: Day of Year

ECA: Ecological Compensation Areas

EO: Earth Observation

EOS: End of Season

EROS: Earth Resources Observation and Science

ETM+: Enhanced Thematic Mapper Plus

EVI: Enhanced Vegetation Index

GIMMS: Global Inventory Modeling and Mapping Studies

GLEL: Geomatics and Landscape Ecology Lab

ISIN: Integrated Sinusoidal

LAI: Leaf Area Index

LEDAPS: Landsat Ecosystem Disturbance Adaptive Processing System

MAX: Maximum

MIR: Middle Infrared

MODIS: Moderate Resolution Imaging Spectroradiometer

MSS: Multi Spectral Scanner

MSR: Mean of Squared Residuals

NASA: National Aeronautics and Space Administration

NDII: Normalized Difference Infrared Index

NDVI: Normalized Difference Vegetation Index

NIR: Near Infrared 
OLI: Operational Land Imager

OSAVI: Optimized Soil Adjusted Vegetation Index

PCA: Principal Component Analysis

RFR: Random Forest Regression

SAVI: Soil Adjusted Vegetation Index

SEAS AMP: Season Amplitude

SEAS INT: Season Integrated

SEAS DUR: Season Duration

SEAS GRE: Season Greenness

SOS: Start of Season

SMA: Spectral Mixture Analysis

SWIR: Shortwave Infrared

SWR: Step-wise Regression

TCT: Tasselled Cap Transformation

TCB: Tasseled Cap Brightness

TCG: Tasseled Cap Greenness

TCW: Tasseled Cap Wetness

TDVI: Transformed Difference Vegetation Index

TM: Thematic Mapper

TS: Theil-Sen

USGS: United States Geological Survey

VI: Vegetation Index

WDRVI: Wide Dynamic Range Vegetation Index 


\section{Chapter 1: Introduction}

\subsection{Biodiversity and its importance in agro-ecosystems}

Biological diversity or the contracted form biodiversity, first used by Lovejoy (1980), is defined as the various forms of life on earth and the variability among living organisms from all sources including, terrestrial, marine and other aquatic ecosystems and the ecological complexes of which they are part (International Convention on Biodiversity, 1992).

In the past few decades, biodiversity has declined significantly, mainly due to human activities. Habitat loss and habitat fragmentation due to land use transformation are major drivers of the recent decline in biodiversity (Fahrig et al., 2013). Large areas of natural habitat worldwide have been transformed to agricultural lands to provide increasing food production. Agricultural lands are simplified ecosystems that reduce plant and animal diversity in favour of commercial production of one or a few plant or animal species (Swift, 2004). In addition, agricultural lands have a lower carrying capacity, which leads to reductions in both the abundance and richness of species at a global scale (Teyssèdre, 2007). Intensive agriculture is a major threat to species at risk. For instance, $60 \%$ of amphibians and birds which are listed in the Red List of Threatened Species by the International Union for Conservation of Nature (IUCN), and between 10 and 20\% of other species in the Red List are threatened by intensive agricultural practices (Norris, 2008).

\subsection{Biodiversity and landscape heterogeneity in agro-ecosystems}

In regions where intensive agriculture and tillage practices are reaching dangerous levels for natural biota, conservational efforts and alternative agricultural practices are being adopted to revitalize the landscape, enhance biodiversity and minimize damage to the living organisms. At the farm level, organic farming, crop rotation, no tillage systems, and management of natural field margins are some of the alternative agricultural practices that promote biodiversity (Yeates et al. 
1997; Hansen et al. 2000; Hald, 1999; Rydberg, 2000). At the landscape level, however, increasing habitat heterogeneity is suggested as a means to enhance species richness and abundance (Benton et al., 2003).

Landscape heterogeneity was first defined as the variation in the horizontal dimension of the landscape (August, 1983). In the landscape ecology literature, it has been discussed with respect to the landscape habitat-matrix binary as well as more spatially and temporally continuous landscape heterogeneity. In the landscape matrix paradigm, the landscape is divided into "habitat", which provides the resources for the species, and the matrix in between habitat patches as "non-habitat" (Haila, 2002). However, this view has been challenged by many authors as they recognized that species perceive the landscape in a much more complex manner and they use more than one land cover type to provide their life cycle requirements (Fahrig et al., 2011). The heterogeneous landscape view has generally replaced the habitat-matrix view to explain the complexity of species response to habitat patchiness. The "habitat heterogeneity hypothesis" (Simpson, 1949; MacArthur and Wilson, 1967) assumes that heterogeneous and complex habitats promote biodiversity by providing more available sources for species requirements such as feeding, nesting, and breeding, as well as providing more diverse niches and dispersal corridors to support a variety of species (Tews et al., 2004). In an agricultural context, a heterogeneous landscape contains more production cover types such as mixed crop types, grazed lands and orchards. In addition, a heterogeneous agricultural landscape is characterized by a complex pattern of mixed field crops, field margins with woody and other natural vegetation, and natural or managed patches of wooded areas, grasslands and wetlands (Bennett et al., 2006). Such patterns are mostly characteristic of traditional farming, whereas in typical modern intensive 
agriculture only a few crops are sown in large uniform fields, often with very little natural or semi-natural cover types (Fahrig et al., 2011).

Habitat heterogeneity in an agricultural landscape is represented by compositional heterogeneity, and configurational heterogeneity (Pasher et al., 2013; Fahrig et al., 2015). Compositional heterogeneity is represented by the number of land cover types or patches in a given spatial extent (cover type richness) and how evenly they are distributed (evenness). Configurational heterogeneity is represented by the size and spatial arrangement of the land cover types or the landscape patterns, often being measured by the mean patch size, edge density, mean patch shape variability amongst other metrics (Cushman et al., 2008; Fahrig et al. 2011; Pasher et al. 2013; Steckel et al., 2014).

In biodiversity studies, different taxonomic groups have been selected to model their response to habitat heterogeneity. They mostly include species of greater conservation importance such as endemic and native or endangered species (Buckland et al., 2013). In agricultural landscapes, small mammals, birds, beetles, spiders, butterflies, bees, aphids and syrphids, have mostly been selected as the target species groups (e.g. Sunderland and Samu, 2000; Galbraith, 1988; Ostman, et al., 2001, Fahrig et al., 2013).

Different species require various operational areas to fulfill their biotic needs (shelter, food, water, breeding, etc.). These areas are highly associated with average species biomass and their ecological role (Korner, 1996). Species with different operational areas are often highly interactive with each other and that interaction and interrelationship is associated with the crossscale nature of the ecosystem (Peterson et al., 1998). The complexity of ecosystem processes across scales affects species interrelationships. Hence, it is a challenge to model species richness 
or the distribution of a single species at a time and more than one species needs to be considered when modeling species response to habitat metrics.

Two factors that affect the response of different species groups to landscape heterogeneity are scale (spatial and temporal; see Section 1.2) and species-specific factors (Fahrig et al., 2003, Oliver et al., 2010). As an example of a species-specific factor, Oliver et al. (2010) discovered the impact of species mobility in the relationship between the population dynamics of butterflies and habitat heterogeneity at multiple spatial scales. They found that habitat heterogeneity has a stronger positive relationship with more mobile butterfly species diversity. In another example, Steckel et al. (2014) emphasized the impacts of trophic level, body size and habitat specialization of bees, wasps and their antagonists in their response to habitat heterogeneity at multiple spatial scales. Bee and wasp diversity was increased by enhancing landscape diversity, while the antagonists' diversity was increased by greater landscape connectivity.

The response of biodiversity to habitat heterogeneity is a scale-dependent ecological phenomenon (Nelson et al., 2007). Scale hereafter refers to spatial scale and is defined as the extent and grain of the data acquired for sample landscapes selected for biodiversity observation and analysis. Extent is the size of the largest existing object or phenomenon (i.e. size of study area), or the largest sampling unit (Dungan et al., 2002; Nelson et al., 2007), while grain is the smallest existing object or phenomenon under study and generally the smallest measurement unit (Dungan et al. 2002).

In addition, seasonal variability in vegetation phenology and temporal changes in landscape patterns may affect the response of biodiversity to habitat heterogeneity. Habitat requirements of species change during their life span. A spatially uniform landscape can fulfill the habitat requirements of species as long as it varies over time in an appropriate way: it either allows the 
species to move between different habitats as needed, or the desired habitat becomes available at the right time due to phenological and structural changes in vegetation cover (Benton et al., 2003). Intensive agriculture promotes simplicity and temporal uniformity by extending the time that the land is in production and reducing the time that the land is not in use. However, spatial heterogeneity in agricultural lands can provide more varying types of habitat for species at the required times in their life cycle.

\subsection{Remote sensing data resolution}

Remote sensing data, available in various spatial, temporal and spectral resolutions, offer tools to map habitat and to model the response of biodiversity to landscape heterogeneity at multiple spatial and temporal scales. In the remote sensing context, spatial scale refers to the extent and grain of images. The coverage area of each image or sampling area within an image is defined as the extent, while spatial resolution is the term that refers to grain. Spatial resolution is defined as the size of the finest ground sample or the grain that is detectable by the sensor and is distinctive from the neighboring samples. The smallest distinctive sample unit is the pixel (Dungan et al., 2002; Bar-Massada et al., 2011). Spatial resolution is a property of the remote sensor. Satellite images may be categorized as low (pixel size $>30 \mathrm{~m}$ ), medium (pixel size $=2.5-30 \mathrm{~m}$ ), or high (pixel size $<2.5 \mathrm{~m}$ ) spatial resolution (Stefanov and Netzband, 2005; Xie et al., 2008; Jones and Vaughan, 2010), although these are arbitrary and other categorizations exist. In multiple spatial scale analysis more than one data type with different coverage and spatial resolution is used to assess, monitor and model biodiversity at regional, landscape and local scales.

Temporal scale in remote sensing refers to the temporal resolution and temporal extent of the image data. Temporal resolution is the frequency of image acquisition or the duration between successive images of the same scene (Jones and Vaughan, 2010). This is constrained by the image 
coverage (larger coverage sensors repeat image acquisitions more frequently), orbit time, number of satellites available for the sensor, and whether more than one image can be acquired for an area during a single pass or on adjacent passes using pointable mirrors. Therefore, temporal resolution varies between sensors from about 30 minutes to weeks or months (Xie et al., 2008). Remote sensing multi-temporal studies and time series analyses use more than a single date image to assess and monitor changes in a landscape. Different data with various temporal resolutions are available for use based on the objective of the study and the required temporal frequency. In addition, temporal extent is the period for which the satellite data is available. For example, time series of Moderate Resolution Image Spectroradiometer (MODIS) data is available since the first launch of this sensor in 1998. Time series of Landsat data is available from 1973 with the launch of Landsat 1 and continues today with the Operational Land Imager (OLI) sensor onboard Landsat 8 , although the pixel size, radiometric sensitivity and spectral bands have changed over successive Landsat systems.

Spectral scale is another property of remote sensors. It is the ability of a sensor to differentiate separate wavelength intervals (the spectral bandwidth). Spectral scale is represented by spectral resolution and extent. Spectral resolution is the bandwidth of each spectral band which often varies with spectral region (for example, visible and near infrared band are usually higher resolution than short wave infrared and thermal infrared for the same image extent), while spectral extent is the coverage of the spectrum by spectral bands. (Jones and Vaughan, 2010). Optical remote sensing images are usually categorized as multispectral or hyperspectral based on their spectral scale. Multispectral images contain a limited number of generally wide bandwidth spectral bands. Hyperspectral images contain many spectral bands with narrower bandwidths and therefore have much more detailed spectral data compared to multispectral images. 
The research of this thesis was designed to model the relationship between farmland biodiversity and habitat heterogeneity and evaluate the potential of multiple spatial, temporal and spectral scale remote sensing data in such modelling.

\subsection{Research goal and questions}

The goal of this research was to determine the response of biodiversity to vegetation quantity and heterogeneity at multiple spatial and temporal scales in mixed agricultural landscapes. The outcomes of the research are expected to be of use in agricultural policy and landscape-farm level efforts through consideration of spatial and temporal dynamics in land cover configuration and composition management for biodiversity enhancement.

In order to detect the biodiversity response to farmland vegetation and its spatial and temporal dynamics, multiple spatial and temporal scale data are required. The research therefore also addresses the broad question related to these two scale types: "Can low spatial and high temporal resolution imagery, and moderate spatial and low temporal resolution imagery be effectively used to create biodiversity models that reflect the response of biodiversity to vegetation and vegetation heterogeneity?" To answer this question, time series of MODIS, with low spatial and high temporal resolution, and time series of Landsat, with moderate spatial and low temporal resolution, were used to create biodiversity models for multiple taxa assemblages at landscape and regional scales.

Many review papers have discussed the applications of spatial and temporal remote sensing data in biodiversity modeling (e.g. Nagendra et al., 2001; Turner et al., 2003; Kuenzer et al., 2014). The studies reviewed were conducted in various habitat types, using a variety of data types and techniques. Many of these studies have been conducted using a single spatial scale and only one type of remote sensing imagery (e.g. Cord et al., 2014; Warren et al., 2014; Duro et al., 
2014, St-Louis et al., 2014). While ecological metrics and landscape variables are scale dependent phenomena, single spatial scale studies did not evaluate the variability in biodiversity response to those metrics at different local, landscape and regional scales. They also did not take advantage of the different spectral and temporal properties of different datasets. Some other biodiversity modeling studies did use multiple remote sensing datasets but did not conduct a long-term multitemporal analysis (e.g. Sheeren et al., 2014). Seasonal, annual and inter-annual variability in the landscape can affect the response of biodiversity to landscape variables. Uni-temporal studies lack a broad temporal dimension and do not take into account the relationship between temporal variability of the landscape and the response of biodiversity. The research of this thesis addresses these issues by creating and evaluating biodiversity models using continuous remote sensing data at multiple spatial and temporal scales. In addition, there is a gap in the literature in modeling multiple species groups in a landscape, as most studies have modeled only one taxon (e.g. Oviler et al., 2010; Flick et al., 2012). However, as mentioned before, various species groups with different operational scales are highly interactive in a context of the cross-scale nature of the ecosystem. Hence, to create a more realistic understanding of biodiversity response to agricultural landscape heterogeneity, more than one taxon should be considered. This research addresses this gap by creating and assessing remote sensing-biodiversity models for multiple farmland species groups including plants, birds, butterflies, syrphids, bees, carabids and spiders.

To address the main research goal, three research questions were designed, which form the core empirical chapters $(4,5$, and 6$)$ of this thesis. These and more specific sub-questions are as follows:

Question 1: Are crop phenology dynamics derived from high temporal resolution, low spatial resolution MODIS intra- and inter-annual time series related to farmland biodiversity? 
- Is there a relationship between crop phenology and local farmland biodiversity when phenology and biodiversity are measured coincidentally (in the same year; 2011-2012)?

- Does the strength of the crop phenology-biodiversity relationship vary when phenology is measured in the years prior (2000-2010) to the biodiversity assessment? If so, is there a pattern showing a lag effect - i.e. phenology in previous years is more related to biodiversity in the present year?

- Is the crop phenology-biodiversity relationship stronger for larger landscape spatial extents?

- Of the seven species groups assessed, do certain groups show consistently stronger relationships with crop phenology?

- From those assessed, what are the most important phenological variables related to biodiversity?

\section{Question 2: Are mid-growing season landscape dynamics derived from low temporal and moderate spatial resolution Landsat inter-annual time series related to farmland biodiversity?}

- Are Landsat-derived indices representing landscape crop productivity, moisture and brightness related to farmland biodiversity assessed in the same years (2011-2012)?

- Are landscape crop productivity, moisture and brightness for individual years from 1985 to 2011 related to 2011-12 farmland biodiversity? i.e., Is there a lag effect in the response of biodiversity to these farmland characteristics?

- Are these landscape-biodiversity relationships stronger for larger landscape spatial extents?

- Of the seven species groups assessed, do certain groups show consistently stronger relationships with crop productivity, landscape moisture or brightness? 
- From those assessed, what are the most important Landsat-derived landscape variables related to biodiversity?

\section{Question 3: Are there inter-annual trends and dynamics in MODIS phenology and Landsat mid-summer landscape metrics, and are these temporal dynamics related to biodiversity assessed in 2011 and 2012 ?}

- Are there inter-annual trends in landscape phenology derived from MODIS time series and is inter-annual variation in phenology related to biodiversity? i.e., Do landscapes with more pixels showing negative or positive trends, or greater inter-annual variability in vegetation phenology have more or less biodiversity?

- Are there inter-annual trends in mid-growing season vegetation, moisture and brightness indices derived from Landsat time series and are inter-annual variations in these indices related to biodiversity? i.e., Do landscapes with more pixels showing negative or positive trends, or greater inter-annual variability in these variables have more or less biodiversity?

- In comparing mid-growing season NDVI time series derived from MODIS and Landsat, which is better for modelling landscape biodiversity?

\subsection{Thesis structure}

This thesis is divided into seven chapters:

Chapter 1 (this chapter) provides a summary of the context related to the biodiversity-landscape heterogeneity relationship and application of remote sensing data for portraying this relationship. Gaps in the literature on biodiversity modeling are discussed, leading to presentation of the overall research goal and three main research questions.

Chapter 2 provides a literature review of the issue of biodiversity in agricultural lands, the impacts of farming practises on farmland species, and the relationship between biodiversity and 
landscape heterogeneity. In this chapter the importance of spatial and temporal scales in modeling the biodiversity-heterogeneity relationship is discussed and the application of multi spatial and multi temporal remote sensing data and techniques in such modeling is reviewed.

Chapter 3 outlines the study area, as well as the biodiversity data and ground sampling protocols that were used to survey species. The remote sensing data utilized in this research are also presented.

Chapter 4 addresses Question 1 and assesses the relationship between vegetation phenology and biodiversity using MODIS high temporal resolution low spatial resolution data. In this chapter the relative effect of sample landscape size on the model performance and the potential of a time lagged response of diversity to phenological dynamics are assessed.

Chapter 5 addresses Question 2 and assesses the effects on biodiversity of mid-summer landscape brightness, vegetation productivity and moisture derived from long-term Landsat 5 data. Similar to Chapter 4, the effects of sample landscape size and time lagged response are assessed.

Chapter 6 addresses Question 3 and assesses the inter-annual landscape trends and temporal dynamics in the phenology and mid-growing season landscape indices derived from MODIS and Landsat time series in Chapters 4 and 5, respectively. The relationships between biodiversity and inter-annual landscape trends and temporal variability are assessed. This is followed by a comparison of MODIS and Landsat inter-annual mid-growing season NDVI time series to determine which data type is best for modelling farmland biodiversity; i.e. it is a comparison of the relative spatial resolutions of these two data types for such landscape scale biodiversity modelling. 
Chapter 7 discusses the overall findings of the research and provides a synthesis of the key themes with respect to the literature. 


\section{Chapter 2: Literature review}

In this chapter, biodiversity and different approaches of measuring biodiversity in a general ecological context are discussed with emphasis on farmland biodiversity. The mutual benefits of farmland species and agricultural productivity are reviewed with various examples from European and North American studies. The importance of studying biodiversity at multiple spatial and temporal scales is highlighted and the application of multi-spatial and multi-temporal remote sensing data and techniques in measuring and modeling biodiversity is discussed.

\subsection{Biodiversity: concepts and relations with agricultural landscape heterogeneity}

Prior to 1990, the study of bioindicators mainly concentrated on indicators of environmental health and services and human induced changes in the environment. However, after the widespread adoption of the term "biodiversity" about 1992, a shift in the literature occurred with more studies focusing on biodiversity itself (Duelli and Obrist, 2003). Biodiversity involves several different and complex aspects, each requiring its own indicators to measure. Based on the initial definition, biodiversity encompasses genetic diversity, species diversity and ecosystem diversity (The International Convention on Biological Diversity, 1992). Many recent ecological studies measure biodiversity at the species level.

Many diversity indicators have been developed based on the fundamental aspects of species diversity (species richness as the number of species in a unit area, species abundance as the number of individuals of a species or taxon, and species evenness represents the relative differences in abundance among taxa; Pielou, 1966) and the interrelation between them. Alpha, beta and gamma diversity indices, developed by Whittaker (1960) are among them. Alpha $(\alpha)$ diversity is the number of species living within an area of a certain size. It therefore is the measure of within-ecosystem species richness. Beta $(\beta)$ diversity describes the degree of changes in species 
composition and abundance along a given habitat or physiographic gradient. Beta diversity is a measure of between-ecosystems diversity; it is expressed as a rate or proportion and demonstrates the species turnover rate (Veech et al., 2002). Finally, gamma $(\gamma)$ diversity was introduced as a measure of overall diversity in a large region that can be measured at the landscape scale (Whittaker, 1960). The appropriate spatial scale to quantify gamma diversity has not been defined, therefore, it can be measured for any scale of interest (Tuomisto, 2010). Gamma diversity is usually calculated as the sum of alpha and beta; however, Whittaker views gamma diversity as the product and not the sum of alpha and beta diversities (Veech et al., 2002). In a landscape context, alpha diversity for each taxon in a landscape is defined as the mean number of species at several locations (sample sites) within the landscape, while gamma diversity for each taxon in a landscape is defined as the total number of species identified across all locations (sample sites) in the landscape and beta diversity is defined as the difference between gamma diversity and mean of alpha diversity (Fahrig et al., 2015).

In addition, Noss (1990) states that biodiversity is more than simply the number of genes, species and ecosystems and most of these definitions do not take into account the ecological processes and interactions, natural disturbances and nutrient cycles. He then introduced three new aspects of biodiversity: compositional diversity, structural diversity and functional diversity. Compositional diversity is defined as the variety of uniquely identifiable elements in a collection, and includes species lists and measures of species diversity and genetic diversity, while structural diversity is the physical organization or pattern of a system and includes measures of pattern of land cover patches at a landscape scale and habitat complexity. Finally, functional diversity involves ecological and evolutionary processes, including gene flow and the range of traits present in the community, which are often responsible for ecosystem function (Franklin, 1988). In other words, functional diversity indicates the ability of an ecosystem to adapt to natural and 
anthropogenic disturbance (Gallardo et al., 2011). Compositional, structural and functional diversity are indicators of habitat heterogeneity. Structural and functional diversity lead to higher compositional diversity, hence simplification of habitat structure and disruption of ecological functions causes loss in compositional diversity (Duelli and Obrist, 2003). Given this association, it is evident that increasing habitat heterogeneity is related to higher species richness and abundance (Moser et al., 2002).

The biodiversity-landscape heterogeneity relationship has been the subject of many ecological studies in various landscapes, including in agricultural lands (Fahrig et al., 2011; Lee and Martin, 2017; Vasseur et al., 2013; Fuhlendorf et al., 2006; Ga'mez-Virue's et al., 2015). About one-third of the world's terrestrial surface is used for agriculture (Herzog et al. 2013). Over the past few decades many agricultural landscapes have undergone rapid and large-scale change mainly due to the agricultural intensification (Donald et al., 2006). Agricultural intensification occurs at a local scale by intensive use of chemicals and other inputs or interventions, and at a landscape scale by destruction of semi-natural habitats and removal of uncultivated patches, shrubs, hedgerows, and isolated trees, hence reducing land cover heterogeneity (Benton et al., 2003). Loss of semi-natural habitats used for nesting, feeding, breeding and refuge for many wildlife species causes a decline in farmland biodiversity (Morelli et al., 2014).

Fahrig et al. (2011) described two components of landscape heterogeneity in the context of agricultural landscapes: compositional heterogeneity as the number of different crop types, and configurational heterogeneity as the spatial pattern of different land cover types. In addition, they defined structural landscape heterogeneity where different cover types are defined by their physical characteristics and functional landscape heterogeneity where different cover types are identified based on resource dependencies of species groups. 
The biodiversity-heterogeneity relationship in farmlands is widely studied across Europe (Zamora et al., 2007; Kleijn et al., 2009; Krauss et al., 2010; Wagner, 2000; Smart et al., 2002; Albrecht et al., 2007; Brose, 2001; Benton et al., 2003). Farmlands cover up to $60 \%$ of the total lands in some European countries (Morellie et al., 2014) and all European Union countries are currently obliged to develop, implement and invest in agri-environmental schemes to protect biodiversity in agricultural ecosystems (Filippi-Codaccioni et al., 2010).

The impacts of land-use intensification on local farmland species have been studied by Haberl et al. (2004) and Kleijn et al. (2009). Haberl et al. (2004) found a strong negative relationship between human appropriation of net primary production and biodiversity of vascular plants, spiders, ants, bees, gastropods, orthoptera and bryophytes in agricultural landscapes of eastern Austria. They defined human appropriation of net primary production as the difference between the potential net primary production of the ecosystem and the net primary production remaining in the ecosystem after harvest i.e. the biomass removed from ecosystem after harvest and change in productivity due to land-use. This confirms that species diversity is negatively correlated with the intensity of land-use. Kleijn et al. (2009) studied the impacts of agriculture intensification, measured as annual nitrogen input, on vascular plant diversity in 271 agricultural fields and grassland fields in six European countries. A significant negative relationship with rare and subdominant plant diversity was found both in the arable lands and grasslands, demonstrating the importance of agricultural management practices in wild plant conservation.

Many European and North American studies have investigated the relationship between habitat heterogeneity and farmland biodiversity. Lee and Martin (2017), studying avian birds in an agricultural region in Mississippi, USA, found no clear pattern between functional diversity and landscape heterogeneity but a strong positive relationship between species richness and landscape heterogeneity. 
Fuhlendorf et al. (2006) studied the relationship between grassland heterogeneity and bird diversity in north-central Oklahoma, USA. They compared bird communities in grasslands with diverse spatial and temporal fire and grazing patterns to those where fire and grazing are applied annually in a spatially uniform pattern. The results showed that fire and grazing disturbances lead to higher vegetation diversity and spatially heterogenous patterns of vegetation and hence a greater variability in grassland bird community. Fire occurrence in patches creates a gradient of vegetation structure more so than uniform fire application, thereby providing more suitable habitat for the different species requirements.

de Simon et al. (2017) studied patterns of plant diversity and habitat compositional and configurational heterogeneity in a mix agricultural landscapes of northeastern Italy. The results showed that natural patches such as meadows, woodlands and hedgerows are plant diversity hot spots and that higher biodiversity and resistance against disturbance are associated with landscapes with higher compositional and configurational heterogeneity.

Morelli et al. (2014) studied the relationship between "high nature value" farmlands and occurrence of bird species in Central Italy. They defined high nature value farmlands as having a high proportion of semi-natural vegetation, low land management intensity, a mosaic of seminatural and cultivated land, and presence of rare species. Of 160 farmlands, more than 50\% were classified as high nature value and they had significantly greater bird species richness than the other farmlands.

Benton et al. (2003) studied the impacts of agricultural landscape heterogeneity across Europe on farmland birds, finding that at multiple special scales (between farms to across regions, between fields and within fields) higher landscape heterogeneity provides diverse habitats for farmland birds and promotes greater species diversity. 
Steckel et al. (2014) studied the impacts of landscape composition and configuration on the populations of bees and wasps (hosts) and their antagonists (arthropods of higher trophic level including three bee species, two predatory beetles, two parasitoid flies and 20 species of parasitoid wasps) in three distinctive locations in Germany. The results show that species richness, abundance and parasitism rate (the ratio between the number of hosts attacked by antagonists per study plot to the total number of hosts per study plot) are enhanced by increasing landscape heterogeneity, however, different trophic levels benefit from different aspects of landscape heterogeneity. Bees and wasps could be supported by enhancing landscape diversity, while the antagonists were found to benefit from greater landscape connectivity.

Moreover, the role and mutual impact of farmland biodiversity in agro-ecosystem health and productivity has been widely investigated. Farmland biodiversity provides various ecosystem services that are essential and beneficial for the well-being of agro-ecosystems and long-term agriculture viability. Non-crop wild plants, invertebrates and farmland birds are some of the most important species dwelling in agricultural lands and contribute to the total farmland biodiversity. Primary production (wild plants), pollination (wild bees, bumblebees and butterflies), predation (spiders and beetles), and decomposition of organic matter (earthworms) are some of the most important ecological functions relevant to farming (Herzog et al., 2013).

Invertebrates, which make up a large number of multicellular organisms, contribute extensively to farmland biodiversity. They reflect the general environmental conditions and quickly react to environmental changes, while they enable direct evaluation of farmland management effects (Filippi-Codaccioni et al., 2010). In addition, natural predators control invertebrate crop pests and allow reduction or even avoidance of chemical pesticides. This control is enhanced by adopting management practices that promote predator biodiversity (Symondson et al., 2002). 
Wild plant diversity within agricultural landscapes is essential in enhancing the functioning and productivity of farmlands and in maintaining ecosystem services in various ways. Maintaining and restoring pollinator diversity, which has an important role in increasing pollinator-dependent crops yields (Carvalheiro et al., 2011; Herzog et al., 2013), as well as pest control and preserving local plant communities against invasive species (Freemark et al., 2001; Albrecht et al., 2007) are some of the services of wild plant diversity in agricultural ecosystems. Restoring wild plant diversity however, involves the creation of areas rich in wild plant diversity (Freemark et al., 2001; Carvalheiro et al., 2011). Given these plants are often considered by farmers as "weeds", reducing the arable land and competing for soil resources with the crop, such practices are often not implemented. In fact, non-crop plants are commonly removed from cultivated fields. The costs, benefits and trade-offs of wild plant diversity in farmlands need to be measured to justify to farmers to need for adoption of farming practices that enhance biodiversity (Carvalheiro et al., 2011).

Harwood et al. (2009) studied spider and beetle species as natural predators of invertebrate crop pests in farmlands of Europe. They found that these predators need to have a diet of diverse prey types to have optimal growth and reproduction. Use of pesticides causes a loss in invertebrate diversity including non-pest invertebrates and hence leads to a reduction in the number of predators. This consequently increases pest numbers and may cause damage to crops. Hence, preserving the diversity of invertebrate prey species within farmlands is essential to allow predators to take advantage of a diverse diet, continue to thrive in crops, and to aid in control of agricultural pests.

Albrecht et al. (2007) studied the relationship between pollinator diversity in "ecological compensation areas" (ECAs) adopted by a Swiss agi-environmental scheme and plant reproductive success. ECAs consist of a variety of land covers such as wildflower strips, orchards, 
hedges and extensively managed hay meadows characterized by postponed mowing and prohibition of fertilizer. They selected pairs of ECAs adjacent to intensely managed meadows and measured and compared the diversity of different pollinator and flower visiting groups such as bees, syrphids, beetles and butterflies. Their results showed that the richness and abundance of pollinators is significantly higher in the ECAs compared to the intensely managed meadows, and the number of small-sized pollinators in intensely managed meadows declined with increasing distance from the adjacent ECA. They also found that the productivity of specific plant types is significantly higher in ECAs than in intensely managed meadows, emphasizing the benefits of the ecosystem services provided by pollinators in more heterogeneous areas.

Carvalheiro et al. (2011) studied the advantages of co-occurrence of wild plant species to the productivity of pollinator dependent crops in sunflower farms. They found that visits and movement of honeybees, as the main pollinators, increase in conjunction with farm productivity when wild plant species co-exist. Their results showed that conservation of natural patches as well as promoting flowering plants within crops maximizes the productivity of pollinator dependent crops.

\subsubsection{Biodiversity and farmland heterogeneity in Canada and the region of this research}

In North America, agriculture is one of the most predominant land cover types and one of the most important means of human-induced natural land transformation. In Canada, agricultural lands make up $7.5 \%$ of the land mass (Committee on the Status of Endangered Wildlife in Canada, COSEWIC, 2004). Canada's agricultural landscape is a combination of cultivated areas and grazing land with associated natural and semi-natural lands including riparian land, wetlands, woodlands and natural grasslands. Over 500 terrestrial vertebrate species including birds, mammals, reptiles and amphibians reside or visit agricultural lands in Canada, a large number that are listed as species at risk by the Committee on the Status of Endangered Wildlife in Canada 
(COSEWIC, 2004). The capacity of Canadian farmlands in carrying wildlife species was relatively stable from 1986 to 1996; however, it declined from 1996 to 2011. Agricultural intensification is the leading cause of a decrease in wildlife habitat capacity since 1996 (AgriEnvironmental Indicator Report Series, 2016).

In Ontario, farming is one of the major economic practices and farmlands are one of the predominant land covers. Agriculture intensification is a leading cause of natural land transformation and a major driver of biodiversity loss in the agricultural regions of Ontario. Wildlife habitat capacity (as a weighted index that relates the number of species using each land cover as primary or secondary habitat for breeding, feeding or cover to the relative proportion occupied by each land cover) decreased in 94\% of Ontario farmlands from 1981 to 2001 (Javorek et al., 2006). The importance of preserving habitat heterogeneity to enhance farmland biodiversity has been emphasized and the heterogeneity-diversity relationship has been largely studied across Ontario farmlands in the past few decades. In addition, the significance of natural and semi natural patches, permanent grasslands and pastures, edges, hedgerows and woody features adjacent to fields in preserving and enhancing biodiversity of farmland species is largely investigated and discussed in the Ontario farmland context.

Freemark et al. (2001) studied the importance of a mosaic of farmland habitats and noncrop patches as refuges for conservation of native plant species in eastern Ontario, Canada. They found the richness and abundance of herbaceous, woody, native and introduced plant species to be more diverse in landscapes with more non-crop habitat. This emphasizes the importance of non-crop buffer strips and woody hedgerows for native plant conservation.

Flick et al. (2012) studied the richness and abundance of butterfly species in an agriculture-dominant area in eastern Ontario. They defined the number of land cover patch types 
or patch richness as the measure of compositional heterogeneity and the total number of individual patches or patch density as the measure of configurational heterogeneity. The results showed that butterfly richness is higher in landscapes with higher patch density while butterfly abundance is higher in landscapes lower patch richness.

Fahrig et al. (2015) studied the relationships of alpha, beta, gamma and abundance of farmland species (plants, birds, beetles, spiders, bees, butterflies and syrphids) with farmland compositional and configurational heterogeneity in agricultural lands of eastern Ontario, using the biodiversity data of this research. They used farmland crop type diversity as the measure of compositional heterogeneity and field size as the measure of configurational heterogeneity. The results showed that mean crop field size has the strongest relationship and overall effect on biodiversity and the relationship is consistently negative for all the taxa studied. This study highlights the importance of smaller fields in enhancing farmland biodiversity.

Monck-Whipp et al. (2018) studied the relationship between landscape heterogeneity and bat diversity in the same landscapes as Fahrig et al. (2015). They hypothesized that more heterogeneous landscapes with various crop types and smaller field sizes enhance the diversity of insects, which are the source of food for bats, and more diverse prey leads to increased bat species diversity. The results showed that bat activity increases significantly in landscapes with more crop types and smaller fields. However, the relationship between bat richness and abundance with landscape heterogeneity was not statistically supported due to the small variability of bat richness and abundance across the sample landscapes.

Wilson et al. (2017) studied the influence of crop heterogeneity and woody structure on bird diversity in agricultural lands of eastern Ontario and southwestern Quebec. They tested the effects of type of agricultural system (forage and pastoral agriculture as more permanent cover 
type with lower tillage and lower chemical input versus arable crops) and linear woody features on avian diversity of agricultural landscapes. The results showed that generalist bird diversity did not demonstrate a relationship with increasing area of forage or arable crops. In contrast, specialist birds such as forest and wetland species declined with increases in the area of arable crops and grassland species increased with increasing area of both forage and arable crops but the relationship was stronger for forage. This study shows the importance of habitat specialization of bird species when modeling relationships between landscape heterogeneity and biodiversity.

\subsection{Spatial and temporal scale of study in biodiversity modeling}

Ecological studies are usually conducted at various spatial scales which are mainly categorized as local, landscape and regional scales. Many studies are directed only at the local and landscape scales (e.g. Concepcion et al., 2008; Culman et al., 2010; Bar-Massada et al., 2011), whereas more comprehensive studies have also conducted regional scale analysis (e.g. Costanza et al., 2011; Steckel et al., 2014). The definition and extent of each scale varies according to the phenomenon or process under study and the objective of the research.

In an agro-environmental context, the response of biodiversity to habitat heterogeneity is studied at three different scales: between-farm (regional) scale, between-field (landscape) scale, and within-field (local) scale. Between-farm heterogeneity has been characterized by a mixed farming system with different configurations of mixed crops and patches of natural land covers at a large regional extent. Between-field heterogeneity has been characterized by a mosaic of different crops linked by natural patches. Within-field heterogeneity has been characterized by the structural variability in vegetation within a field caused by variability in a seed set, grazing or edaphic factors (Benton et al., 2003). 
In addition, in order to gain insight about changes in ecosystem structure, ecological processes and dynamics, and ecosystem services, ecosystems are often studied at more than one point in time. Long-term studies are usually defined as those which continue longer than the generation time of the dominant species in the ecosystem (Strayer et al., 1986). They can detect abrupt natural events and quantify important phenomena that are not identifiable with a crosssectional approach, as well as distinguish short-term intra or inter-annual fluctuation from longterm trajectories. Furthermore, long-term studies can identify vulnerable species and the effectiveness of conservational practices (Lindenmayer et al., 2012). Long-term biodiversity research investigates the abundance and variability of species at a given location at several points in time, based on the temporal turn over in a community (Magurran et al., 2010).

In an agricultural context, temporal research scales may include within-season and between season changes representing the phenological cycles in crops and other vegetation, as well as annual and inter-annual changes in crops and farmlands. Phenological changes in farmlands due to seasonal and year-to year weather and climate or due to the presence of various crops with different growth cycles and different emergence and senescence times may be related to the richness and abundance of species but this has not been well studied. Hence, measuring crop seasonal, annual and inter-annual temporal variability is essential to determine the relationship between crop phenological changes and the richness and abundance of local farmland species.

Many biodiversity modeling studies have been conducted on multiple spatial and temporal scales to test the effect of scale on species response to habitat metrics. For example, Mooreno and Halffter (2001) studied diversity of bat species in a mixed forest and agriculture area in Veracruz, Mexico. They conducted bat sampling at multiple time periods to test the effect of time on alpha, beta and gamma diversity. Zamora et al. (2007) studied the alpha, beta and gamma diversity of 
dung beetles as an indicator group to identify the most important habitats for biodiversity conservation in a traditional Mediterranean agricultural landscape in the north of Alicante province, Spain. They conducted sampling at the landscape scale and local (among habitats) scale every fifteen days over the course of one year (from May 2001 to June 2002) to test the effect of spatial scale at seasonal and intra-annual temporal extents. Cunningham et al. (2014) studied woodland bird diversity response to habitat metrics at the landscape, farm and within-farm scales from 2002 to 2010 in southeast of Australia. They tested the relationship between bird species richness with landscape metrics including the total area of woody vegetation, the number of vegetation patches and the average and standard deviation of each vegetation patch area, as well as changes in bird diversity over time related to changes in the landscape. Guyot et al. (2017) studied the species-specific habitat (breeding site, migration stop-over and winter habitat) of bird communities in vineyards of southwestern Switzerland at landscape and field scales during different seasons. At the landscape scale, they tested the effect of semi-natural habitats within the agricultural matrix while at the field scale they tested the importance of vegetation structure and vineyard management for birds. They also evaluated season-specific effects of habitat characteristics on bird foraging activities.

\subsubsection{Lag effects of landscape and habitat change on biodiversity}

A simple way to examine abrupt or gradual temporal changes in biodiversity is to plot biodiversity indicators against time (Magurran et al., 2010). However, biodiversity response to physical or biotic changes in habitat is not often immediate and involves considerable time lags (Essl et al., 2015). Loss of biodiversity and extinction processes occur with a time delay and those populations close to their extinction threshold might survive for a long time before they go extinct (Krauss et al., 2010). At both regional and local scales, actual extinction rates can be surprisingly lower than estimations, given the large extent of habitat loss (Hylander and Ehrlen, 2013). This time-delayed 
loss in biodiversity is also referred to by ecologists as "relaxation time" (Diamond, 1972) and "extinction debt" (Tilman et al., 1994).

Different mechanisms have been suggested as driving factors of time-delayed biodiversity response. Habitat loss and habitat fragmentation, as dominant features of environmental change, are well-studied (Krauss et al., 2010; Tilman et al., 2001; Hahs et al., 2009) since they are relatively straightforward to quantify by evaluating changes in habitat extent using historical data. Other factors that have been studied include: ecosystem degradation, change in ecosystem connectivity as a consequence of habitat loss, climate change, change in disturbance regime, changes in biotic interactions, successional changes, changes in biophysical processes, selective removal of species, species transport or invasion, evolutionary changes, and other adaptive changes (Essl et al., 2015). The species body size, longevity, habitat specialization and trophic level are some of the important factors in showing a time lag in response to habitat changes (Krauss et al. 2010).

Various studies of this phenomenon at regional and local scales have focused on single or multiple taxonomic groups, some of which reported evidence of a time lag whereas others did not. Krauss et al. (2010) studied time-delayed loss in biodiversity due to habitat loss and fragmentation in 147 semi-natural grassland patches across Europe. They surveyed two trophic levels (habitat specialized vascular plants and butterflies) from 2000 to 2007 and modeled them against past (based on historic aerial photos taken from 1950 to 1960) and present (based on aerial photos taken from 1999 to 2007) habitat area. They found that habitat-specialized vascular plants, but not butterflies, showed time-delayed extinction. Their model parameters showed that past habitat area is the most important predictor for the current vascular plant richness. In contrast, for butterflies the current landscape area was the most important predictor of species richness. 
Chen and Shen (2017) created models to predict the delayed responses of species diversity and community structure to habitat destruction in local communities. The results showed that time-delayed responses to changing habitat area occurred for some species in local communities, while endemic and rare species did not experience extinction debt.

Sales et al. (2015) studied the effect of forest patch size on the time lag of response of abundance of two forest primate species (black penciled marmoset Callithrix penicilatta and black-fronted titi monkey Callicebus nigrifrons) in the Brazilian Atlantic forest. The results demonstrated that marmosets with small body size and rapid reproductive rates, which can occupy several habitat types, respond fast to habitat change and do not show a time lag, whereas titi monkeys with slower reproduction rates and longer generation time display possible lag effects.

Dullinger et al. (2012) modeled 150 high-mountain plant species across the European Alps. The model predicted an average range size reduction of $44-50 \%$ by the end of the $21^{\text {st }}$ century due to climate change; however, the model also indicated that plant population dynamics will lag behind the climate trend, thereby creating an extinction debt.

Findlay and Bourdages (2000), studied the temporal response of birds, mammals, vascular plants and herptiles to road construction in wetlands of southeastern Ontario. They found that biodiversity was negatively related to road density in wetland areas, but this response was not immediate. A strong negative correlation was found between diversity of vascular plants, birds and herptiles with historic road construction, showing that effects of road construction on some taxa may not be detectable for decades.

\subsection{Biodiversity modeling using remote sensing}

Remote sensing offers data and tools for derivation of Earth surface reflectance and its spatial and temporal characteristics as well as to map landscapes and habitat classes for modelling, 
assessment, and monitoring of the landscape heterogeneity-biodiversity relationship. It creates opportunity for interdisciplinary work that advances both the ecology and remote sensing fields. Recent advancements in satellite technology and the launch of multiple spatial, temporal and spectral resolution remote sensors led to an increasing trend in using remote sensing tools for mapping landscapes and modeling the biodiversity response to habitat and habitat change. The application of remote sensing in biodiversity-heterogeneity modeling is widely studied and has been reviewed in several papers and books (e.g. Nagendra et al., 2001; Turner et al., 2003; Franklin, 2009; Kuenzer et al., 2014). Turner et al. (2003) categorized two main approaches under which the remote sensing biodiversity modeling research has been conducted:

1) The direct approach is a first order approach that detects the occurrence of individual species (Turner et al., 2003). Kuenzer et al. (2014) state that landscape thematic classes such as vegetation types and landscape texture such as the size and shape of ground objects are also directly detectable variables. The direct approach has two main limitations. First, the target species that are able to be detected are limited to large-crown trees in forested areas or smaller plants in a uniform contiguous area (Duro et al., 2007). Second, the data that could be used to detect individual species are limited to the high spatial resolution imagery (Nagendra et al., 2001; Turner et al., 2003). Given many vegetation types overlap in space, this approach is generally best suited to mapping vegetation communities or species assemblages related to habitat type and biodiversity.

2) The indirect approach acquires or derives habitat or environmental variables believed to affect the distribution of the species and applies those variables in models of biodiversity. Many species are limited by variables such as climate, topography and available resources. Climate variables (e.g., seasonal temperature, relative humidity and soil moisture), topographic variables (e.g., elevation, slope and aspect), primary productivity of the habitat (as a net indicator of vegetation 
cover), and remote sensing data-based index variables (e.g., brightness, greenness (vegetation) and wetness indices) are examples of indirect variables (Turner et al. 2003 and Kuenzer et al. 2014).

The indirect approach does not have the limitations of the direct approach and can be used to model the abundance and diversity of any taxon for which appropriate habitat-environment variable data can be acquired (Kuenzer et al. 2014). There is also no limitation in terms of the type of data or imagery that can be used in the indirect approach since, depending on the objective of the research and the amount of required detail, various spatial, temporal and spectral resolution images could be used.

\subsubsection{Scale and sensor considerations}

The spatial resolution of remote sensing data is important in modeling the biodiversityheterogeneity relationship. Spatial scale can affect the way that the habitat phenomena and processes are perceived by the species (Tews et al., 2004). Those phenomena and processes are not always studied at an appropriate scale, though. For instance, habitat heterogeneity is a landscape scale phenomenon which had mostly been studied at a local or patch scale (Fahrig et al., 2003). When a combination of remote sensing and field data is used for biodiversity modeling, choosing the right remote sensing data with pixel size smaller than the minimum ground sampling unit is essential to ensure that the spatial scale between the remotely sensed and the biodiversity field data are matching (Turner et al., 2003; Rocchini et al., 2010).

The advantages and drawbacks of different remote sensing spatial resolutions in mapping heterogeneous landscapes for biodiversity modeling are widely discussed (Rocchini et al., 2010; Nagendra et al., 2013). Unlike uniform habitats, more heterogeneous landscapes with fine-grain continuous land cover patches are more challenging to map accurately. It is often assumed that 
higher spatial resolution performs better in mapping habitats, however, the optimal spatial resolution depends highly on the extent of species' distributions and habitat heterogeneity as well as the availability of ancillary datasets such as soil, topography and climate.

The relative advantages and tradeoffs between spatial and spectral resolution should be taken into consideration when choosing remote sensing data for habitat mapping and biodiversityheterogeneity modeling. Rocchini et al. (2010) and Nagendra et al. (2013) suggested that it is best to prioritize spectral resolution even when spatial resolution is compromised. For instance, some high spatial resolution sensors such as IKONOS and Quickbird have lacked middle infrared (MIR) and thermal infrared bands and this has reduced their capabilities in identifying land cover or habitat types that are sensitive to these bands, whereas, the MIR and thermal infrared bands of the lower spatial resolution Landsat sensor capture this important spectral information.

The temporal variability in vegetation and landscape patterns is also important in modeling the biodiversity-heterogeneity relationship as noted in Section 2.2. Biodiversity studies using remote sensing are categorized as uni-temporal, multi-temporal and time series studies, which enable biodiversity monitoring during a defined period of time (from daily to monthly, seasonal, annual, inter-annual and even decadal time spans (Kuenzer et al., 2014)). Many studies have used remote sensing data from the past 40 years to analyze or map phenological changes in vegetation or species diversity. Phenology is often analyzed using time series of vegetation indices. They are derived by combining reflectance measurements from different portions of the electromagnetic spectrum in mathematical formulations based on the relationships between low reflectance in the visible due to pigment absorption, high reflectance of vegetation in the near infrared (NIR) due to scattering of radiance by leaf cell walls, and absorption by moisture in the shortwave infrared (SWIR). Most traditional vegetation indices are based on the red-NIR relationship (Baig et al., 2014). For Example, the Normalized Difference Vegetation Index 
(NDVI) and Enhanced Vegetation Index (EVI) acquired throughout the growing season by sensors with high acquisition frequency. NDVI and EVI are based on the absorption of red wavelength by chlorophyll and other leaf pigments, and the strong reflection of near-infrared wavelength by foliage. NDVI and EVI are measured by the equation 1 and 2 respectively:

$$
N D V I=\frac{(\mathrm{NIR}-\mathrm{RED})}{(\mathrm{NIR}+\mathrm{RED})}
$$

(Equation 2.1)

$E V I=\mathrm{G} \frac{(\mathrm{NIR}-\mathrm{RED})}{(\mathrm{NIR}+\mathrm{C} 1 \times \mathrm{RED}+\mathrm{C} 2)}$

(Equation 2.2)

where NIR and RED are atmospherically-corrected surface reflectances, C1 and C2 are the coefficients of the aerosol resistance term, and $\mathrm{G}$ is the gain factor.

NDVI time series with better than weekly frequency have been available since the 1970s at coarse pixel size (over $1 \mathrm{~km}$ ) from the Advanced Very High Resolution Radiometer (AVHRR) and since 1999 at 250m pixel size with the Moderate Resolution Image Spectrometer (MODIS). Both have been used to study vegetation phenological changes along with additional indices such as EVI generated from MODIS (Nagendra et al. 2013; Boyd and Foody 2011). Despite the low resolution of AVHRR and MODIS, their high temporal resolutions and large spatial coverage make them appropriate data for multi-temporal habitat mapping and biodiversity-heterogeneity modeling at landscape to regional scales.

Multiple spatial and temporal scale remote sensing data have long been used in ecological studies. Using multiple spatial resolutions allows evaluation of the impacts of scale-specific ecological phenomena or processes over a variety of extents. Multiple temporal resolution data enables evaluation and monitoring of the impacts of seasonal, annual and inter-annual changes in the ecosystem. Using multiple sensors with different spectral characteristics also provides different spectral information that may be of relevance to the habitat of given species or taxa. 
Categorization of ecological applications of remote sensing, including biodiversityheterogeneity modeling and habitat mapping, based on spatial and temporal resolution are as follows: 1) Multi-spatial and uni-temporal resolution (Doraiswamy et al. 2004, Stefanov and Netzband 2005), 2) Multi-temporal and single spatial resolution (Gómez et al. 2011, Nitze et al. 2014, Zhu et al. 2014), and 3) Multi-temporal and multi-spatial resolution that integrate high spatial resolution with high temporal resolution datasets (Costanza et al. 2011, Jia et al. 2014, Li et al. 2014).

\subsubsection{MODIS and Landsat time series for analysis of vegetation dynamics}

Multi-spatial and multi-temporal scale studies attempt to capture the spatial variability of complex landscapes and the temporal changes in land cover, particularly in vegetation. The archives of high temporal resolution MODIS and moderate spatial resolution Landsat, both operated by NASA and publicly available free of charge, are among the most popular sources of remote sensing data in multi-spatial and multi-temporal studies. As they were the sensors used in this research, they are both briefly summarized below with example vegetation, habitat and biodiversity applications that incorporate spatial and/or temporal information.

MODIS is a key instrument aboard the Terra and Aqua satellites launched in 1999 and 2002 , respectively. Both are sun synchronous and pass from north to south across the equator; Terra passes in the morning while Aqua passes in the afternoon. Orbit time is 99 minutes so they view the entire Earth surface every 1 or 2 days. MODIS has 36 spectral bands. The nominal pixel size for bands 1 and 2 (band width $620-670 \mathrm{~nm}$ and $841-876 \mathrm{~nm}$ respectively), used in this research, is $250 \mathrm{~m}$, while for bands 3-7 (band width between 459 and $2155 \mathrm{~nm}$ ) and 8-36 (band width between $405 \mathrm{~nm}$ and $14.385 \mu \mathrm{m}$ ) the spatial resolution is 500 and $1000 \mathrm{~m}$, respectively (www.modis.gsfc.nasa.gov). 
The high temporal resolution of MODIS enables the capture of weekly to seasonal phenology while the 20-year temporal extent allows analysis of annual to decadal vegetation dynamics. It has significantly advanced the mapping of spatial and temporal landscape patterns at a relatively high level of spatial detail for such a global scale sensor (Dubovyk et al. 2015). The radiometric and geometric properties of MODIS products along with the improved atmospheric correction and cloud removal make them an appropriate and easy to use temporal dataset. In addition to the spectral bands, MODIS provides calibrated and sensor specific vegetation indices, including the previously mentioned NDVI and EVI, as well as other products (e.g., Surface Reflectance, Land Surface Temperature and Emissivity, Fraction of Photosynthetically Active Radiation (FPAR), Leaf Area Index (LAI), amongst others; www.modis.gsfc.nasa.gov). MODIS vegetation indices have been derived from well calibrated reflectance data at high temporal frequency and have significantly advanced capabilities to accurately capture vegetation phenology variables such as the time of onset of greenness and senescence, growing season peak productivity, and spatial-temporal variations in canopy structure (Nitze et al. 2014).

Applications of MODIS NDVI time series have been both in inter-annual and intra-annual phenological analysis of vegetation change and dynamics. An example of inter-annual vegetation analysis using MODIS NDVI time series is Wang et al. (2013) who mapped the distribution of Crofton weed in southwest China using a multi-stage method based on its inter-annual spectral variability in relation to other vegetation land covers (forest and pasture). Examples of applications of MODIS NDVI time series in intra-annual vegetation analysis include the following: Perry et al. (2014) modelled and estimated dryland wheat biomass in Victoria, Australia. They investigated the relationship between time series of MODIS NDVI and in situ measured and simulated above ground biomass, LAI and fractional green cover to create an integrated crop growth model. These models showed the potential of MODIS NDVI time series 
and crop simulation models in regional vegetation mapping. McInnes et al. (2015) differentiated native and non-native (tame) grasslands, which are important in terms of their relative capabilities to support biodiversity in the Dry Mixedgrass region of Alberta, Canada. The two classes were found to have different rates of spring green up at the pixel level, which could be separated using a linear discriminant function. Classification of MODIS NDVI time series produced a much more reliable identification of the different grassland types compared to traditional remote sensing techniques and aerial photo interpretation.

Landsat acquires higher spatial resolution data that can capture more spatial detail and smaller scale landscape patterns than MODIS (Dubovyk et al. 2015). The Landsat program was first launched in 1972; Landsat 1-3 carried the Multi Spectral Scanner (MSS) instrument with 4 multispectral bands in the visible and near infrared, $79 \mathrm{~m}$ nominal pixel size and an 18 day repeat coverage. Subsequent Landsat instruments included the Thematic Mapper (TM), carried on Landsat 4 and 5, the Enhanced Thematic Mapper Plus (ETM+) carried on Landsat 7, and the Operational Land Imager (OLI) carried on Landsat 8. Each provided improved spectral properties (narrower bandwidths, better calibration), more spectral bands (including the addition of MIR and TIR bands), and improved spatial resolution. The historical archive of Landsat TM, ETM+ and OLI, the repeat coverage of every 16 days, and the nominal pixel size of $30 \mathrm{~m}$ provide potential to map temporal changes in a given landscape within a given growing season if cloud free data exist, and more certainly inter-annually over a period of up to four decades. The spectral properties of Landsat enable analysis of information about vegetation greenness and productivity as well as soil and vegetation moisture content.

Application of Landsat data in ecological studies has been intensive over its full period of operation. Time series of Landsat have been extensively used in inter-annual vegetation change studies. In forest applications, studies have focused on gradual forest disturbance, deforestation 
and land conversion, as well as abrupt changes such as harvest, wild fire and insect defoliation. Examples of temporal data analysis include the following. Huang et al. (2010) used Landsat TM and ETM+ time series from 1984 to 2006 to detect reconstruction of forests in USA after going through disturbances due to harvest and fire. They used change detection algorithms and time series analysis techniques based on the spectral-temporal properties of forest, disturbance and post-disturbance recovery processes to detect the temporal changes and the disturbances that occurred during the period under investigation. Kennedy et al. (2010) used Landsat TM and ETM+ time series from 1985 to 2007 to track changes in a set of randomly located sample forest patches in Pacific Northwest of USA. They captured both short-duration events and long-term trends by extracting temporal trajectories of spectral data on a pixel by pixel basis. They used a temporal segmentation method to accurately capture a wide range of disturbances and recovery phenomena over time. Roder et al. (2008) used time series of Landsat MSS, TM and ETM+ from 1975 to 2000, to monitor fire events and post fire dynamics of a forest area southwest of Valencia, Spain. They used Spectral Mixture Analysis (SMA) to derive estimates of active green vegetation as a primary indicator and applied a trend analysis technique to characterize post-fire dynamics and succession patterns. Nguyen et al. (2016) used Tasseled Cap Transformation (TCT) on Landsat TM, ETM and OLI from 1989 to 2014 to map mangrove forests in Vietnam and detect changes by comparing maps over the study period. They found an approximately $20 \%$ loss of mangrove forests from 1989 to 2014 mainly due to land conversion, while approximately 44\% of the area of the mangrove forests was newly plants or rehabilitated. Misurec et al. (2016) studied the spatio-temporal changes of Norway spruce stands in a mountainous area in the Czech Republic using Landsat TM, ETM+ and OLI from 1985 to 2015. They extracted the general trends in forest disturbance and recovery using TCT and vegetation indices. The results detected moderate to heavily damaged areas in 1998, while an improvement in forest health status was 
shown in 2013. Vogelmann et al. (2012) analyzed stacks of Landsat TM and ETM+ time series from 1987 through 2010 to detect vegetation changes in four different study sites (mixed forest and rangeland, sagebrush-dominated rangeland, woodland adjacent to prairie, and forest) in the US. They used NDVI and the short-wave infrared (SWIR) to near infrared (NIR) ration index (SWIR/NIR or Band 5/Band 4) to assess trends. The results displayed a gradual systematic change in many natural vegetation communities in all four areas. For example, in southwestern US many of the coniferous forests showed declines due to insect and drought. Similarly, many sagebrush communities showed decreases in greenness due to fire, mining and drought, while in Nebraska forest communities showed local expansion and increased canopy densification. This study provided evidence that temporal trends were not detectable through casual inspection of Landsat images, and time series analysis of multiple scenes acquired in multiple years is required to develop an appropriate representation of patterns and trends of ecosystem changes. These studies are examples of the potential of Landsat time series in detecting changes and trends in land cover and ecological phenomena.

In agricultural applications, studies have focused on crop mapping and detection of patterns in farming practices such as fallow fields and irrigation. For example, Liao et al. (2015) studied the spatio-temporal dynamics of swidden agriculture and fallow vegetation recovery in Laos using the Landsat TM and ETM+ archive over 20 years. They classified Landsat time series from 2000 to 2010 using a decision tree classifier followed by a spatio-temporal analysis of changes in swidden agriculture. They subsequently delineated the dynamics of the burning and cropping phase in all images and identified the burned pixels in the 2000 image by comparing to the corresponding pixels in the following years. They investigated temporal trends, land-use frequency, and the swidden cycle using time-series of Landsat NDVI. The results showed an increase of nearly 55\% in the area of swidden agriculture in northern Laos from 1999 to 2011. 
Feng et al. (2015) studied cropland dynamics of the Yellow River Delta in China using Landsat TM, ETM+ and OLI from 1986 to 2015. Comparison of random forest classifications of land cover for each year showed an increase of approximately $251 \mathrm{~km}^{2}$ in cropland area from 1986 to 2015 as well as losses due to cropland abandonment and urban sprawl. Martinez et al. (2005) used a time series of Landsat TM and ETM+ from 1993 to 2000 to map cropping patterns in a small irrigation district in northeast of Spain. They used maximum likelihood classification to map crop types and then analyzed the spatio-temporal changes in the crop patterns. Vieira et al. (2012) used Landsat time series acquired in spring (September and October) 2000 and summer (February and March) 2001 to classify and map the distribution of sugarcane farms in Sao Paulo, Brazil. They used object-based image analysis and data mining techniques for Landsat TM and ETM+ image classification and they were able to accurately map ready-to-harvest sugarcane areas in the study region.

Some studies have used both MODIS and Landsat data in multiple spatial and temporal scale vegetation analysis for comparison or validation purposes, or to complement each other. In forest applications, studies focused on detecting inter-annual forest phenology. For example, Fisher and Mustard (2007) used MODIS and Landsat time series to analyze the phenology of forest areas in Massachusetts (Harvard forest) and New Hampshire (Hubbard Brook). They compared the phenology measured by Landsat (30 $\mathrm{m}$ resolution) with the phenology measured by MODIS (500 $\mathrm{m}$ resolution) and found similar model strengths for estimation of greenness onset $\left(\mathrm{R}^{2}=0.60\right)$. They subsequently used MODIS estimation of phenology to generate interannual trajectories from 2000 to 2005. They compared MODIS estimations to historic records and found that MODIS successfully predicts $86 \%$ of the variance at Harvard forest and $70 \%$ of the variance at Hubbard Brook. 
In agricultural applications, Landsat and MODIS have been combined for crop classification, identification of crop patterns and evaluation of crop condition. For example, Li et al. (2014) used multiple dates of MODIS and Landsat to classify crop types in an agricultural region in Hungary. They used Landsat images acquired in August and MODIS images acquired in March to September 2011, finding that classification accuracy improved when both datasets were combined. Doraiswamy et al. (2004) used a combination of Landsat and MODIS data to assess crop physiological condition and its integration in a crop yield simulation model. They used Landsat data for land use and crop classification and MODIS for development of specific parameters such as LAI. The Landsat-based land use map and the MODIS-based LAI simulated image were then coupled with ancillary soil and climate data to create separate soil moisture and yield maps. The LAI simulation was repeated for different dates from early July to end of August to capture different stages of crop development and subsequently the soil moisture and crop yield maps were created for each stage of the growing season.

Modelling the biodiversity-heterogeneity relationship in an agricultural context has been done using a combination of MODIS and Landsat datasets. For example, Costanza et al. (2011) used MODIS and Landsat time series from 2001 to 2007 to evaluate the relationship between local plant richness and heterogeneity in North and South Carolina, US. They measured heterogeneity at multiple special scales: within-plot scale using filed plots, within-habitat scale within the habitat patches surrounding the field plots, neighborhood scale within the area across these patches, and region scale where the field plots were located. Heterogeneity metrics were extracted from Landsat and MODIS NDVI as a proxy of productivity for within-habitat, neighborhood and regional scales. For within-habitat and neighborhood scales, NDVI data from 14 Landsat TM images from the growing seasons of 2000-2002, and for the regional scale the mean NDVI of each pixel from the MODIS sensor from 2001 to 2007 was used as an aggregate 
representation of long-term patterns of productivity. They found that the heterogeneity metrics show stronger relationships with local biodiversity at scales larger than within-plots.

\subsubsection{Remote sensing approaches in biodiversity modelling}

A combination of multi-spatial and multi-temporal scale remote sensing datasets has been used in several biodiversity-heterogeneity modeling studies. Land cover classification is the most common approach to create discrete representations of land surface from which landscape heterogeneity is characterized. Numerous land cover classification techniques have been developed and applied in habitat mapping and biodiversity modeling (reviewed by Nagendra et al. 2001; Turner et al., 2003; Gottschalk et al., 2005; Gillespie et. al., 2008; Wang et al., 2010; Petrou et. al., 2011). These techniques generally classify each pixel as a single land cover class and link them to a particular spatial structure. However, discrete classification does not take into account the mixed and continuous nature of remote sensing data and the spectral variability within pixels (Rocchini et al., 2010; Cord et al., 2014). Such 'hard' classification generates abrupt thresholds and distinct boundaries and fails to capture the subtle internal variation within a class, the gradual changes between classes in transitional zones, and the continuity of landscape patterns (Rocchini et al., 2010; St-Louis et al., 2014; Duro et al., 2014). Development of 'soft' classifiers (e.g. fuzzy (Foody, 2002) and spectral unmixing (Wang et al., 2016)) has improved capabilities to detect and map gradients. However, vegetation and land cover maps derived from classification techniques still represent an abstraction that aggregates the original reflectance data. They are also often generated for a specific purpose that is not representative of the target species or taxa (Cord et al., 2014).

Alternatively, the use of continuous reflectance information based on the pixel-to-pixel spectral variability has been suggested (Nagendra, 2001). Rocchini et al. (2010) describe "spectral heterogeneity" as the effects of spatial heterogeneity on the spectral signals received at the pixel 
level. The spectral variation hypothesis (Palmer et al., 2000) suggests that spectral heterogeneity over space is associated with vegetation variability and is a proxy for biodiversity. Different measures of spectral heterogeneity have been developed based on this concept. Measures of dispersion are the simplest and can be applied to any spectral band. The simplest are the mean and variance of spectral reflectance in a given area, for example within the $n \times n$ pixels in the immediate neighborhood of each sampling unit or location (Rocchini et al., 2010). Band combinations such as vegetation indices as can be used to create continuous representations of vegetation composition and structure. NDVI is often used as a metric representing above ground primary productivity of a landscape and has been associated with biodiversity and species distribution (Nagendra, 2001; Gillespie et al., 2008). Spatial variability in NDVI can indicate the variability in vegetation cover type and/or quantity, and therefore serves as an indicator of spatial heterogeneity. First order (e.g., standard deviation) and second order texture (e.g. co-occurrence texture; St. Louis, 2006) of NDVI are examples of metrics that measure local or regional NDVI variability.

Band transformation techniques such as Principal Component Analysis (PCA) and the Tasseled Cap Transformation (TCT) were developed to reduce the dimensionality of multispectral datasets and to aid identification of spectral gradients associated with physical environmental gradients and processes (Jensen, 2015). They commonly represent generalized scene components with unique spectral reflectance gradients such as: bare soil/urban land covers which results in moderate to high reflectance across the visible-NIR-MIR; vegetation which produces low visible reflectance, high NIR reflectance and moderate MIR reflectance; and water/wetness which has low visible reflectance and negligible NIR and MIR reflectance. These continuous indices can also be used in habitat mapping and biodiversity modeling. 
PCA is a linear transformation technique that takes the original set of potentially correlated numerical variables with high covariance and creates smaller and uncorrelated sets of variables that reduce redundancy (Sing, 1989). TCT is also a linear transformation technique that was originally derived for the four bands of Landsat MSS data to understand plant growth patterns in the spectral space (multi-dimensional axes) formed by different band combinations (Kauth and Thomas, 1976). It was found that reflectance data distributions in the red and NIR spectral space for landscapes with varying quantities of vegetation from none to very dense was shaped like a tasseled cap (Figure 2.1). The top of the tasseled cap with low red and higher NIR reflectance was representative of dense vegetation, while the flat side of the cap was representative of wet bare soil (low reflectance in both bands) to dry bare soil (high reflectance in both bands) (Ray 1994). Later, when the Landsat TM sensor with SWIR bands was developed (1982), the SWIR axis was added and TCT was utilized to detect and analyze soil and vegetation moisture and other hydrological features. Ecological research using TCT has evolved and has been performed using data from different satellite sensors including Landsat TM and OLI (Landsat 8), MODIS, QuickBird and IKONOS. TCT is now used for multiple ecological applications including investigating crop condition and land cover classification (Laspanora and Masini, 2007). 


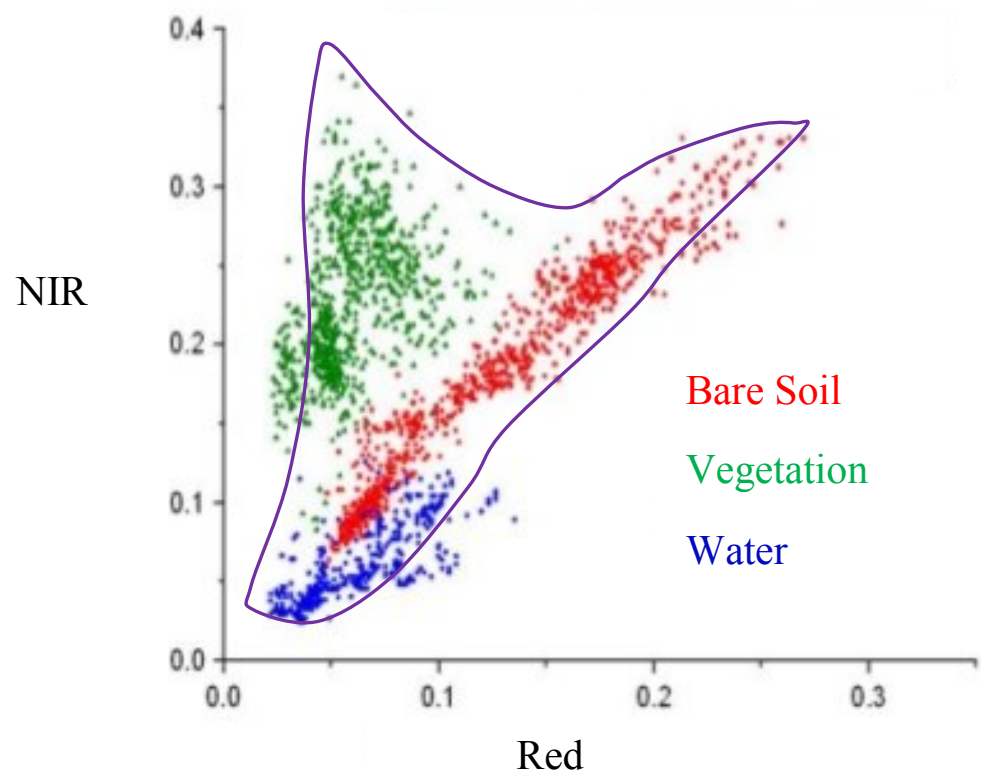

Figure 2. 1. Data distribution in Tasseled Cap Transformation (adopted from Kauth and Thomas (1976))

Similar to PCA, TCT transforms the image data to a new coordinate system with a new set of orthogonal axes. Brightness is generally the primary axis, represented by the weighted sum of reflectances over all spectral bands and typically accounting for the most variance in the scene. It is associated with bare or partially covered soil as well as man-made features such as concrete, asphalt, gravel, and other bare areas (Baig et al., 2014). The second axis is generally associated with the amount of green vegetation and is called Greeness. Wetness is the third component and is associated with a moisture gradient soils and vegetation through to conditions of standing water (Kauth and Thomas, 1976). TCT was first designed to detect changes in vegetation and urban areas and now is used in a broad range of ecological applications including crop monitoring, forestry, and ecosystem management.

Remote sensing ecological studies that have used continuous metrics are generally more recent than those that have used classification for thematic mapping. Examples of studies that 
used remote sensing continuous metrics for modeling biodiversity are as follows. Sheeren et al. (2014) used continuous NDVI data to model bird species distribution in a forest and agriculture mixed landscape in Southwest France. They used WorldView 2, SPOT 4, SPOT 5, Landsat and MODIS NDVI data to compare models generated with the different spatial resolutions of these data types. The results proved the effectiveness of continuous NDVI data and demonstrated that SPOT 5 (10 m spatial resolution) generated the best model for their study site. Warren et al. (2014) used different spectral metrics derived from IKONOS as potential indicators of plant species richness and landscape heterogeneity in a military training area in Germany. Spectral metrics included the mean, standard deviation, number of unique values, and Shannon index of unique values for: 1) reflectance in the four bands, 2) compressed data statistics (11-bit original data compressed to 20 and 100 values, and 3) the first three principal components. Models showed that the number of unique values in the first principal component had the strongest relationship with both the number of habitat patches as the measure of habitat heterogeneity and plant alpha diversity as the measure of plant species richness. The relationship was stronger for habitat heterogeneity than species richness. Cord et al. (2014) used the Maximum Entropy algorithm applied to time series of MODIS EVI over an 8-year period to study phenological variations and predict the distribution pattern of 30 tree species in a forested area in Mexico. They also compared this model to that produced from a discrete land cover map, finding that the continuous data model performed significantly better. St-Louis et al. (2014) applied the spectral unmixing technique as a measure of within-pixel habitat composition along with NDVI image texture as a measure of among-pixel heterogeneity to model avian biodiversity in a semi-arid mixed landscape in New Mexico using Landsat data. The results demonstrated that the NDVI image texture model or the measures of among-pixel habitat heterogeneity outperformed the spectral unmixing model or measures of within-pixel habitat composition. 
Some studies have compared discrete and continuous metrics in biodiversityheterogeneity modeling and habitat mapping. For example, Chust et al. (2002) used Landsat TM continuous reflectance data (NDVI) and discrete land cover maps to extract landscape descriptors to test the response of soil fauna diversity to landscape heterogeneity at multiple spatial scales in Western Europe. They emphasized that Landsat-derived NDVI metrics demonstrated a stronger capability to portray habitat structure than land cover maps and that at a regional scale, habitat plays an important role in structuring species composition, which may not be evident in smaller scales.

In a study that preceded this research but used the same field biodiversity data, Duro et al. (2014) used Landsat NDVI and five NDVI-derived statistical measures (Mean NDVI, Mean range of NDVI in summer, and local NDVI spatial variance metrics: CV, Geary's C, and Moran's I) to assess the effectiveness of continuous NDVI metrics in modeling the diversity of birds, butterflies and plants in agricultural lands of eastern Ontario, Canada. They compared the model derived from the NDVI metrics with a model derived from discrete land cover classes that included mean field size, percentage of cropped area per landscape and Shannon diversity index of cropped vegetation. The results indicated that the NDVI model outperformed the model generated from a discrete land cover map and that spatial image information was as important as spectral information. The results of this study, and of those above, informed the decision in this research to take a direct reflectance-based analytical approach as opposed to a classification approach to modelling biodiversity using multiple spatial and temporal scale remote sensing data. 


\subsection{Summary}

This chapter defined biodiversity and different approaches of measuring and modeling biodiversity in various landscapes, as well as the importance of agricultural landscapes as a reserve for biodiversity of farmland species and the benefits of farmland species for crop productivity. The reviewed studies emphasized the interrelationship between farmland biodiversity, crop productivity and agricultural landscape heterogeneity in the European, North American, Canadian and eastern Ontarian context. They demonstrated that heterogeneous landscapes provide diverse habitat for farmland species and promote biodiversity.

The importance of studying ecological phenomena at multiple spatial and temporal scales was discussed and potential lag effects in the response of biodiversity to changes in habitats were presented. The reviewed papers illustrated the effect of study spatial extent on the biodiversityheterogeneity relationship. The strength of this relationship was also found to be determined by species-specific factors such as body size, trophic level and habitat requirements. In addition, many papers discussed the importance of multi temporal investigation of the biodiversityheterogeneity relationship to model time lagged response of species to landscape changes. These studies showed that the response of some species groups or taxa to disturbance was not immediate and indicated a lag, while no response lag was not evident for other groups.

Remote sensing data and techniques have been found to be useful in modeling biodiversity-heterogeneity relationships at multiple spatial and temporal scales. The papers reviewed illustrated the applicability of high temporal resolution imagery such as MODIS and moderate spatial resolution data such as Landsat, and a combination of both, in ecological mapping and biodiversity modeling. 
The literature revealed the main findings to-date and the current status, as well as the gaps and research needs in multi-spatial and multi-temporal remote sensing for farmland biodiversity modeling. As mentioned in Chapter 1.2, a gap in the literature was found in investigating the effects of annual and inter-annual vegetation phenology and dynamics, i.e. temporal heterogeneity on farmland biodiversity. On the other hand, the effect of multiple spatial scales on the biodiversity-heterogeneity relationship and its association with temporal heterogeneity have not been largely studied. Most of the reviewed studies were conducted at a single spatial scale and/or at a single point in time and/or focused on only a single taxon. This research was designed to address these issues by conducting multi-spatial and multi-temporal analyses for modeling the relationships of biodiversity of multiple taxa with landscape spatial and temporal dynamics. 


\section{Chapter 3: Study site and data}

In this chapter the study site and data used in this research are described. In Section 3.2 the geographic location of the study area, predominant land cover types in the region as well as main crop types, and the sample landscape selection protocol are described. Examples of different sample landscape types (combinations of high and low compositional and configurational heterogeneity) are presented.

Section 3.3 describes the data that were used in this study, including the biodiversity data, the surveyed species and the data collection protocols implemented in 2011 and 2012 (Section 3.3.1), and the remote sensing data (Section 3.3.2). The low spatial, high temporal resolution MODIS images that were used to create crop seasonal and phenological models are described in Section 3.3.2.1 followed by the moderate spatial and low temporal resolution images of Landsat TM5, which were used to create long-term inter-annual vegetation models.

\subsection{Study site}

The study area is located within Eastern Ontario, south of the City of Ottawa. It is approximately $10,000 \mathrm{~km}^{2}$ and is located within the Mixedwood Plains ecozone (Environment and Climate Change Canada, 2014). The climate of the region consists of relatively warm summers (mean daily temperature of $18^{\circ} \mathrm{C}$ to $22^{\circ} \mathrm{C}$ ) and cool winters (mean daily January temperature of $-3^{\circ} \mathrm{C}$ to $-12^{\circ} \mathrm{C}$ ) (Environment and Climate Change Canada, 2014). According to the 2011 agricultural census, approximately $50 \%$ of the land cover of the region was crops and pasture, while approximately $38 \%$ were wooded areas. The study area is situated within Prescott and Russel United Counties, Leeds and Grenville United Counties, and Stormont, Dundas and Glengarry United Counties. This region was selected as the study area because it is the largest continuous agricultural area in proximity to the research centre (Carleton University, Ottawa). This proximity 
facilitated the intensive field biodiversity surveys in 2011 and 2012 as well as acquisition of high resolution aerial photos for creating land cover maps of the study landscapes prior to this study. Subsequently, field visits were conducted for this research to update the land cover maps in spring and summer of 2015. The study area was well known and had been used in previous research of the Geomatics and Landscape Ecology Lab (GLEL) at Carleton University, including as part of an NSERC Strategic Project.

According to the Ontario Ministry of Agriculture and Food, in 2011, the first year of biodiversity data acquisition, $7.13 \%$ of farms in Ontario were located in this region, with the majority reporting a size under 53 hectares. Conventional farming techniques are the dominant agricultural practices in the region. The agricultural lands are mainly covered by maize (21\%), soybean (19\%), forage crops (alfalfa, clover, hay; 30\%), and wheat (3\%) (Ontario Ministry of Agriculture and Food, 2011).

Prior to this study, 120 sample landscapes were selected in this region, as part of an NSERC Strategic Project. Sample landscapes were selected to match the approximate mean size of the farms (about 100 ha) and the range of crop types and field sizes in the region (Pasher et al., 2013; Fahrig et al., 2015). They were selected based on spatial heterogeneity metric information derived from object-based classification of Landsat-5 data (30 m pixels) from 2007. Mean patch (field) size was used as a configurational heterogeneity metric and Shannon crop diversity index was used as a compositional heterogeneity metric. The location and extent of the sample landscapes were determined using a multi-stage spatial analysis (Pasher et al., 2013). Landscape locations were selected based on a 1 x $1 \mathrm{~km}(100 \mathrm{ha})$ moving window procedure that considered all possible landscapes and selected 120 locations that had $60-90 \%$ agriculture land cover and maximum variability between them with respect to the heterogeneity metrics. The optimal landscape extent was determined by evaluating extents ranging from $1 \times 1 \mathrm{~km}$ to $7 \times 7 \mathrm{~km}$. The 
coefficient of variation (CV) of the heterogeneity metrics decreased with increasing landscape extent and levelled off at about $5 \times 5 \mathrm{~km}$, suggesting that this extent was the maximum that should be considered. Very high correlations between $5 \times 5 \mathrm{~km}$ and $4 \times 4 \mathrm{~km}$ landscape metrics, as well as between the $4 \times 4 \mathrm{~km}$ and $3 \times 3 \mathrm{~km}$ metrics suggested that the heterogeneity metrics calculated for $3 \times 3 \mathrm{~km}$ sample landscapes were representative of heterogeneity out to about $5 \times 5 \mathrm{~km}$. In addition, a sample size of 120 was not feasible using $4 \times 4 \mathrm{~km}$ or $5 \times 5 \mathrm{~km}$ landscapes as several landscapes would overlap. Hence, $3 \times 3 \mathrm{~km}$ was selected as the maximum spatial extent. In addition, the minimum distance between the sample landscape centres that had low spatial autocorrelation in the two heterogeneity variables was calculated as $3.5 \mathrm{~km}$. Finally, landscape selection was also based on minimizing the correlation between mean field size and Shannon crop diversity (Pasher et al., 2013).

The selected landscape land cover types were mapped within the central $1 \times 1 \mathrm{~km}$ using field observations made at the time of the biodiversity surveys and $40 \mathrm{~cm}$ pixel aerial photos. Land cover was mapped out to $3 \times 3 \mathrm{~km}$ using the aerial photos and field observations from roadways. During the aerial photo interpretation and land cover mapping, some of the selected landscapes were eliminated from further analysis, as field and air photo mapping showed they did not meet the criterion of $60-90 \%$ agricultural land cover. In addition, during the biodiversity surveys in 2011 and 2012, some landowners did not grant land access to the research team, therefore a few other landscapes were eliminated and the final number was reduced to 93. For generalization purposes, each sample landscape was labelled as one of four categories: low compositional and low configurational heterogeneity (LL), low compositional and high configurational heterogeneity (LH), high compositional and low configurational heterogeneity (HL), high compositional and high configurational heterogeneity (HH) (Pasher et al., 2013; Fahrig et al., 2015). They are shown in Figure 3.1 and an example landscape in each of the four 
categories is given in Figure 3.2. For more details on the landscape selection process, see Pasher et al. (2013). For the analysis of this thesis only $3 \times 3 \mathrm{~km}$ as the maximum landscape extent selected in Pasher et al. (2011) and $1 \times 1 \mathrm{~km}$ extent as the minimum extent at which the biodiversity survey was conducted were used to test and compare the relative effect of sample

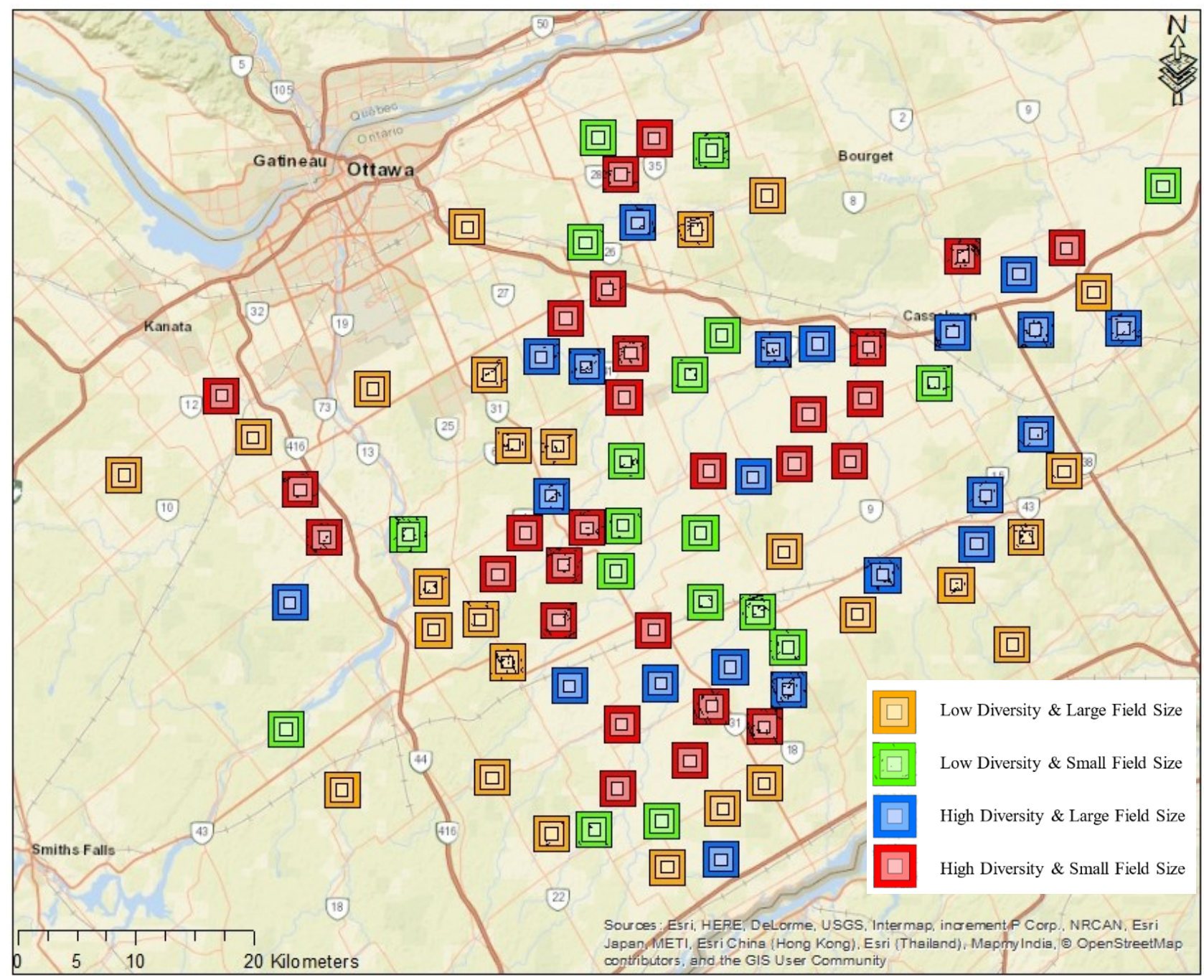

Figure 3.1. The sample landscapes. Different square sizes indicate the 1, 2 and $3 \mathrm{~km}$ extent of each landscape, and colours represent the different categories of landscape compositional and configurational heterogeneity (Adapted from Pasher et al., 2013). 


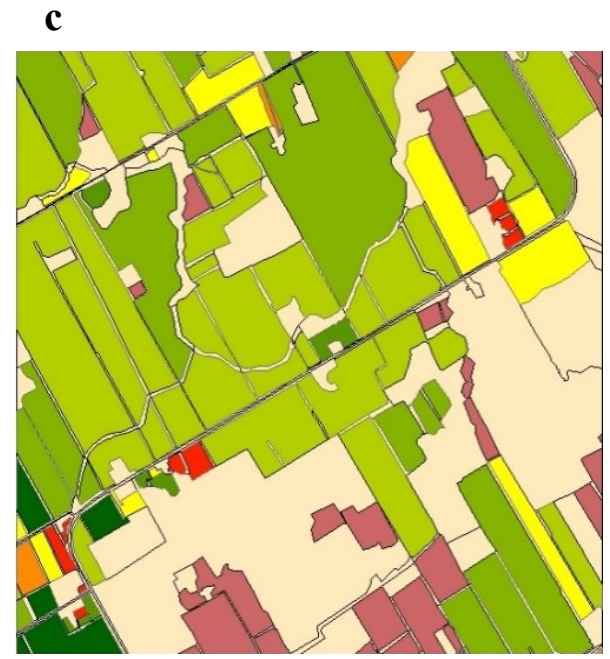

b

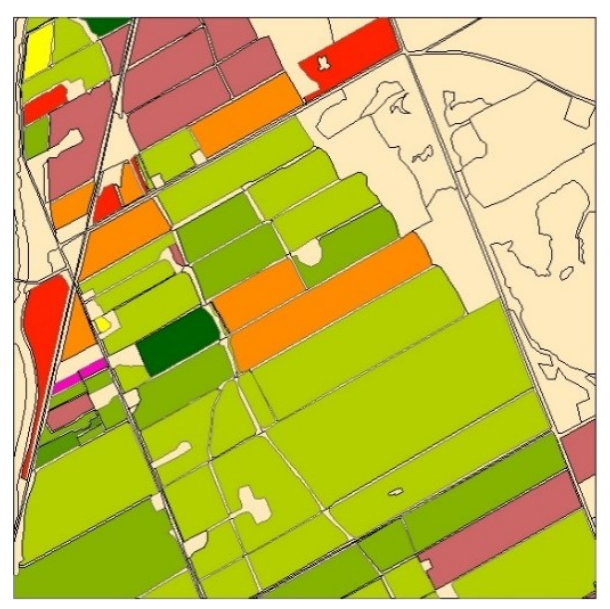

$\mathbf{a}$

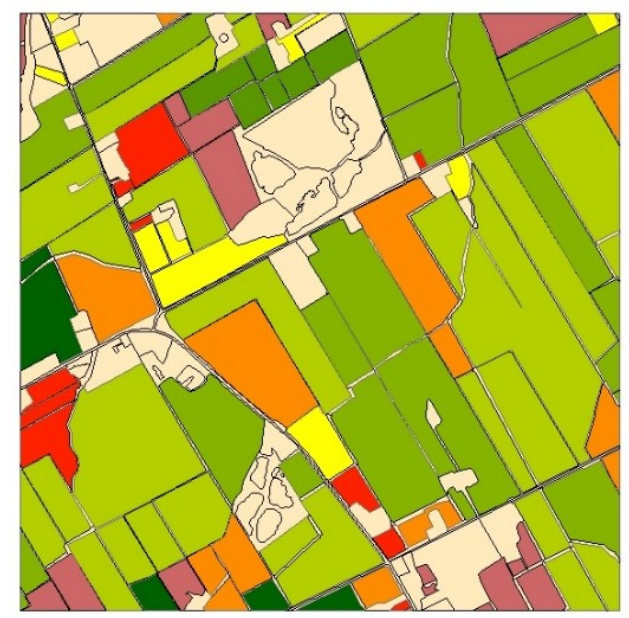

d

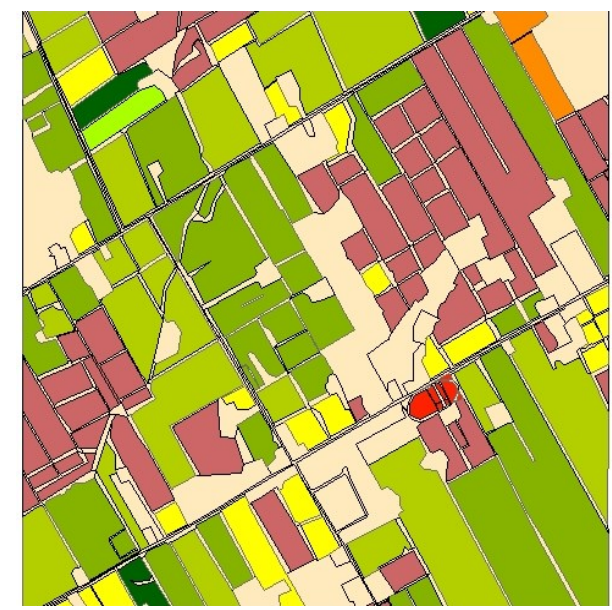

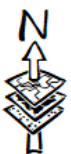

Legend

CropType

cereal

$\square$ corn

fallow

$\square$ hay

legumes

$\square$ mixed vegetable

$\square$ notcrop

pasture

soybean

strawberry

unknown

Figure 3.2. Example $3 \times 3 \mathrm{~km}$ landscapes representing a) high compositional-high configurational heterogeneity (H_H_18), b) low compositional-low configurational heterogeneity (L_L_204), c) high compositional-low configurational heterogeneity (H_L_35), and d) low compositional-high configurational heterogeneity ( $\mathrm{L}_{-} \mathrm{H}_{-}$78).

\subsection{Data}

\subsubsection{Biodiversity data}

Biodiversity was surveyed prior to this study in the cropped portions of the 93 sample landscapes, of which 46 landscapes were surveyed in 2011 and the remaining 47 landscapes were surveyed in 2012. Details of the surveys are given in Fahrig et al. (2015) and summarized here as they form the core biodiversity data used in this research. The surveys were completed in the central $1 \times 1$ $\mathrm{km}$ extent of each sample landscape at four points that were at least $200 \mathrm{~m}$ apart and $50 \mathrm{~m}$ from 
both non-crop areas and the edges of the landscape. Seven species groups were surveyed, namely birds, plants, butterflies, beetles, syrphids, bees, and spiders.

Birds were surveyed using 10-minute point counts at each of the four sample points. They were surveyed from May to July of both years between sunrise and four hours after sunrise which is the best time that singing birds could be heard. All farmland birds which were heard or seen within $100 \mathrm{~m}$ were recorded.

Plants were recorded at each of the four sample points per landscape. At each of the sample points, one of the two adjacent crop fields was selected randomly and plants were counted along a $50 \mathrm{~m}$ transect associated with each point and placed parallel to the field boundary, about $25 \mathrm{~m}$ into the crop (Figure 3.3). The surveyor recorded all plant species present at the four sampling point and within $1 \mathrm{~m}$ on either side of the transect. Plant surveys were carried out along each transect between May 24 and July 9 (identified only non-woody plants) and between July 18 and August 26 (identified woody plants in edge transects as well as edge features) of both years and the species lists from the two surveys at each site were combined.

Butterflies were recorded at the sample points and transects that were used for plant surveys. They were surveyed by walking along the $50 \mathrm{~m}$ plant transects at a rate of $\sim 5 \mathrm{~m} / \mathrm{min}$. Surveys were conducted first between June 27 and August 3 and again between August 4 and August 31 of both years (surveys at the same site were at least 12 days apart). All butterflies were identified
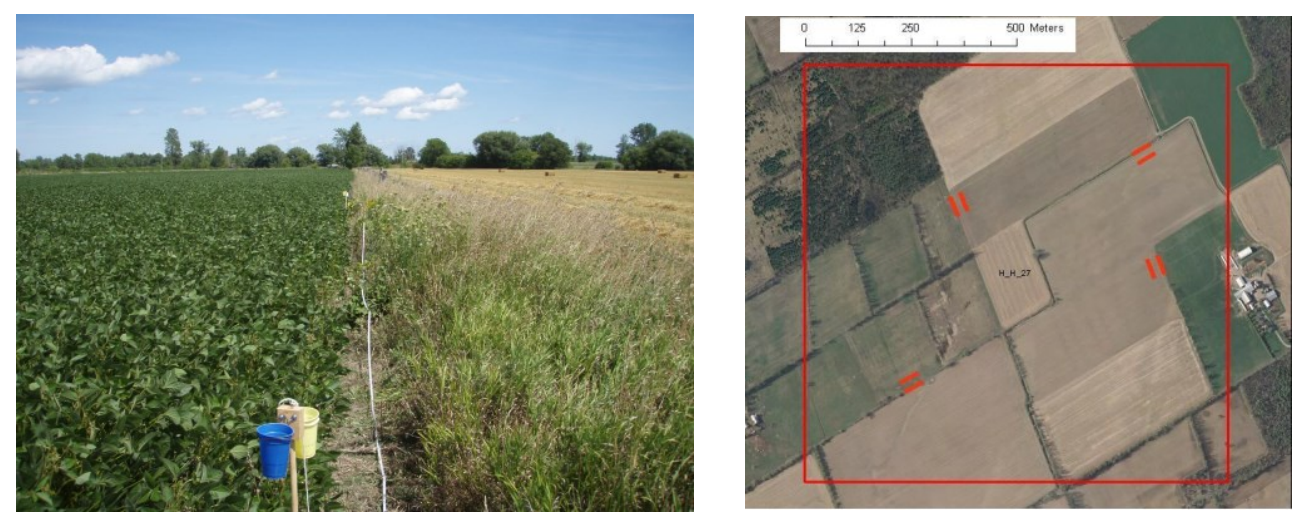

Figure 3.3. Examples of survey transects used for plant as well as butterfly, syrphid, bee spider and beetle surveys. Bee pan traps appeared in the left photo. Photo courtesy of Jude Girard, 2012. 
by sight within an imaginary $5 \mathrm{~m}^{3}$ box $(2.5 \mathrm{~m}$ on each side of the transect and $5 \mathrm{~m}$ in front of the surveyor) as the observer moved. All butterflies not immediately identifiable were captured by a butterfly net of $38 \mathrm{~cm}$ diameter (Figure 3.4) for closer examination (Fahrig et al. 2015). Abundance and species lists were summed over the two surveys at each site.

Syrphid flies were surveyed immediately after the butterfly surveys using the same protocol, but within an imaginary $2 \mathrm{~m}^{3}$ box in front of the observer. Abundance and species lists were summed over the two surveys at each site.

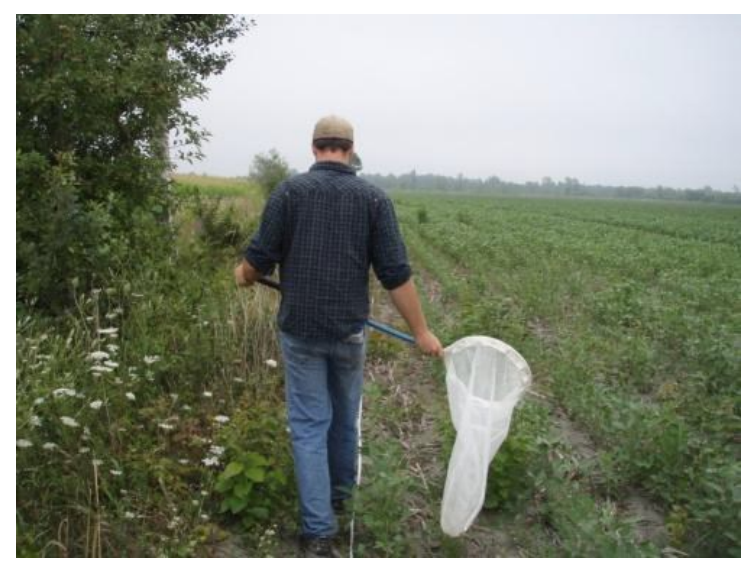

Figure 3.4. Capturing butterflies for identification by a butterfly net of $38 \mathrm{~cm}$ diameter. Photo courtesy of Jude Girard, 2012.

Beetles and spiders were surveyed using pitfall traps at the same points per landscape that were used as the basis for the butterfly and syrphid surveys. Trapping was carried out for two periods of four days each at each landscape between June 15 and July 24 and again between July 24 and August 31 of both years. Pitfall traps were placed in the ground at each end of each plant transect, such that the top edge of the trap was level with the ground (Figure 3.5). Traps were 15 $\mathrm{cm}$ high with a 9-cm diameter opening and were half-filled with water and dish soap (10 drops per litre). A lid of approximately $20 \mathrm{~cm}^{2}$ was place over each trap, approximately $3 \mathrm{~cm}$ above the ground, to protect the trap from rain. Abundance and species lists for each group were summed over the two surveys at each site. 

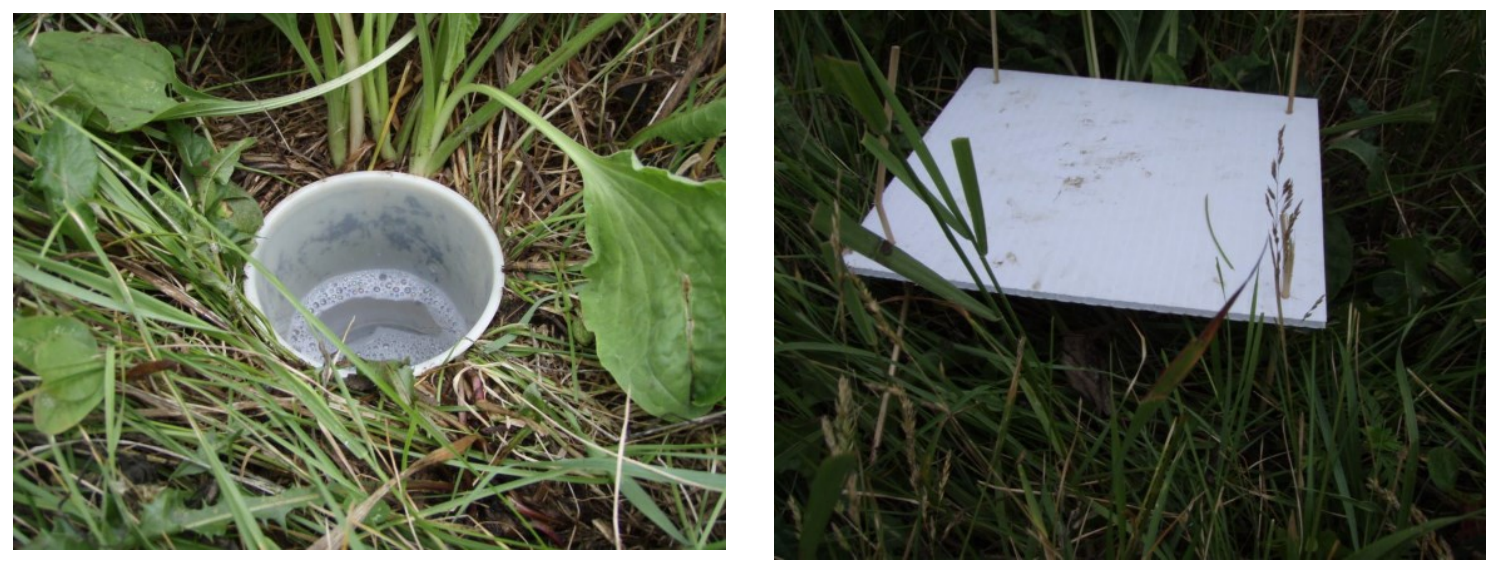

Figure 3.5. Ground beetle and spider traps. Left: the pitfall trap; right: the trap cover. Photo courtesy of Jude Girard, 2012.

Bees were only surveyed in 2011 using six pan traps at each of the butterfly sample points in the landscape. Two pan traps (coloured plastic cups) were placed on the tops of three poles with adjustable height. The poles were placed at the two ends and the centre of the plant transect. Pole height was adjusted to be even with the height of the surrounding vegetation, allowing approaching bees to see them (Figure 3.6). Traps were $2 / 3$ filled with water and dish soap (10 drops/l) to break the surface tension. The pans were in place for four days each during the same two periods as the butterfly and syrphid surveys. Abundance and species lists were summed over the two surveys at each site.
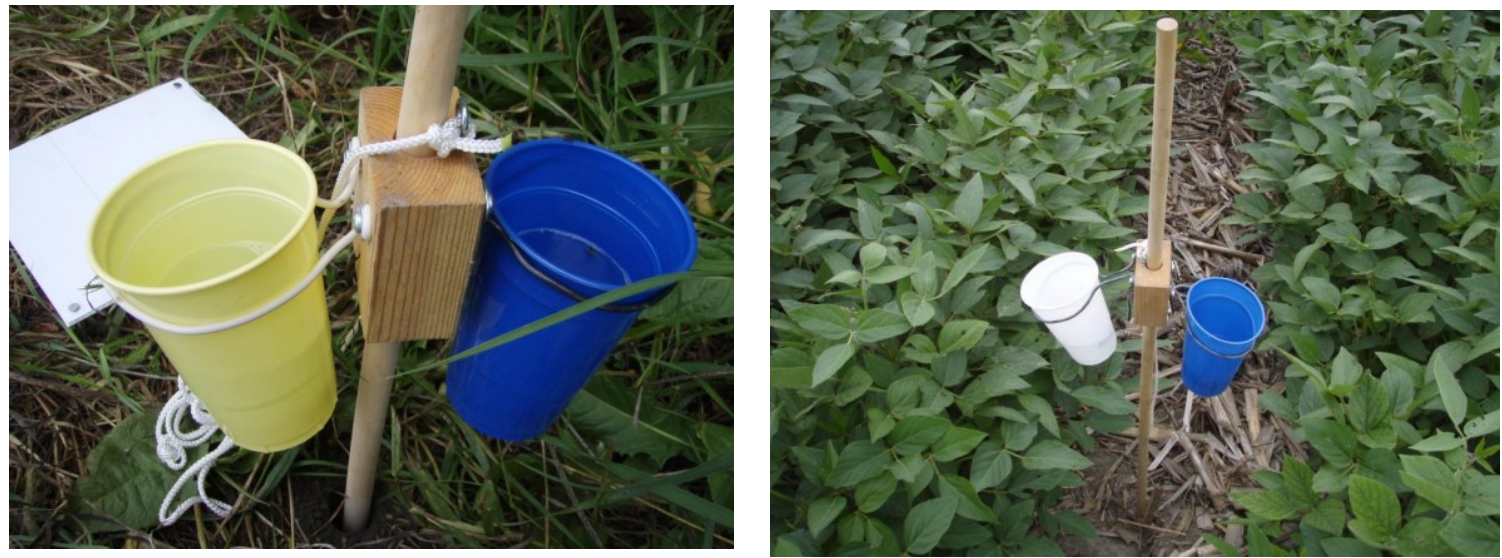

Figure 3.6. Bee pan traps. Photo courtesy of Jude Girard, 2012.

These species groups represent a broad range of potential responses to landscape metrics and patterns. Altogether species counts were: 52 birds, 227 plants, 30 butterflies, 40 syrphids, 58 bees, 
80 carabids, and 98 spiders (Fahrig et al., 2015). The lists of the species recorded are presented in Appendix I.

The mean alpha, beta and gamma diversity values (as defined in Chapter 2.1) per sample landscape were calculated. Alpha diversity was calculated as the mean number of species recorded at each of the sample points within the $1 \times 1 \mathrm{~km}$ extent, while gamma diversity was calculated as the total number of species identified over all sample points within the $1 \times 1 \mathrm{~km}$ extent. Finally, beta diversity, which is a measure of species replacement or movement, was calculated as [gamma diversity - mean alpha diversity] (Fahrig et al., 2015). There were no significant differences (t-test) between sampling years (2011 and 2012) for the means of alpha, beta, and gamma for any of the taxa.

\subsubsection{Remote sensing data}

\subsubsection{MODIS data}

Data acquired by high temporal resolution sensors with short return periods (1-2 days) enable monitoring of seasonal and phenological variations in vegetation within the growing season. This monitoring is primarily based on image time series of vegetation indices (VIs), particularly NDVI derived from sensors such as AVHRR or MODIS.

The spatial, temporal and spectral properties of MODIS are described in Section 2.3. For this study, time series of MODIS weekly Maximum-NDVI from 2000 to 2015 were provided by the Canadian Ag-Land Monitoring System (CALMS) of AAFC. CALMS data are used by AAFC to monitor temporal changes in vegetation phenology at a regional scale. CALMS is implemented weekly throughout Canada's growing season. The growing season is defined as the six-month period between the start of Julian week 12 (end of March) to the end of Julian week 43 (late October). The CALMS MODIS weekly Maximum-NDVI products are generated in the MODIS 
native Integrated Sinusoidal (ISIN) projection and cover the area of Canada from $60^{\circ} \mathrm{N}$ to $40^{\circ} \mathrm{N}$. CALMS integrates three MODIS products to generate various NDVI-based products: 1) MOD09GA, which provides Bands 1-7 in a daily gridded level-2 product, including $500 \mathrm{~m}$ pixel reflectance values and $1 \mathrm{~km}$ observation and geolocation statistics; 2) MOD09GQ, which provides Bands 1 and 2 in a daily gridded level-2 product with 250 m pixels; and 3) MOD10A1 (MODIS/Terra Snow Cover) in a daily gridded level-3 product with $500 \mathrm{~m}$ pixels (Davidson, 2015). In this study, the Best-Quality Maximum-NDVI product was used in which each pixel value corresponds to the best quality Maximum-NDVI that has been recorded within that pixel over the specified week. It is generated in 15 steps, each implemented by running an individual Python module is the CALMS toolset. These steps are presented in Appendix II.

\subsubsection{Landsat data}

In order to model the relationship between farmland biodiversity and long-term inter-annual changes in vegetation, Landsat 5 surface reflectance data with 30 m pixels were downloaded from the Landsat Ecosystem Disturbance Adaptive Processing System (LEDAPS). LEDAPS atmospheric correction is described in Appendix II. Data from 1985 to 2011 with less than 5\% cloud cover were used. The dates (presented in Chapter 5.3) were selected to be within the expected range of dates corresponding to the mid-summer peak of the growing season (JulyAugust). Vegetation indices and the Tasseled Cap Transformation were calculated as described in Chapter 5.1. 


\section{Chapter 4: Modeling the relationship between vegetation phenology and local biodiversity of agricultural lands in Eastern Ontario using MODIS time-series data}

\subsection{Introduction}

Phenology is defined by the U.S. International Biological Program Phenology Committee as "the study of the timing of recurring biological events, the causes of their timing with regard to biotic and abiotic forces, and the interrelation among phases of the same or different species." (White et al., 2009). Crop phenology is most often associated with agricultural events such as planting, emergence, fruiting, and harvest (Reed et al., 1994). Seasonality on the other hand, is defined as "the occurrence of certain obvious biotic and abiotic events or groups of events within a definite limited period or periods of the astronomic (solar, calendar) year" (Lieth, 1974). Seasonal characteristics of plants, such as emergence and senescence, are highly associated with atmospheric factors, such as temperature, precipitation and the variation in annual cycle of weather pattern (de Beurs and Henebry, 2010). Therefore, year-to-year variation in phenological events can be an indicator of climate variation or larger scale environmental change. Some of the factors that influence crop phenology include photoperiod, soil moisture, soil and air temperature, and solar illumination. In addition, short term and extreme weather events such as excessive precipitation and abnormally cold and wet spring seasons affect the timing of major phenological events such as emergence and senescence (Reed et al., 1994).

Monitoring of vegetation phenology has been traditionally conducted by field observations which is costly, time consuming, required personnel and other resources, and has limited observational scope (Zhao et al., 2015). Remote sensing technology can measure and monitor vegetation phenology over large areas and long time periods in a cost effective manner. Satellite analysis of phenology, however, is fundamentally different from traditional ground- 
based observations. The satellite sensor's capability to directly detect important phenological events such as budding and flowering of individual plants is limited due to sensor spatial and temporal resolution and the effects of other ground features such as non-crop vegetation and soil background. However, they are well suited to detection and estimation of large extent changes and phenological variability across the landscape.

High temporal resolution satellite imagery such as Advanced Very High Resolution Radiometer (AVHRR) and Moderate Resolution Imaging Spectroradiometer (MODIS), with a 12 day repeat cycle have been used for detection and measurement of crop phenological changes (Hurlbert and Haskell, 2003; Vina et al., 2016; Thompson and Paull, 2017; Wang et al., 2016). Time-series of vegetation indices, especially the Normalized Difference Vegetation Index (NDVI) and Enhanced Vegetation Index (EVI) derived from AVHRR and MODIS data have been used for acquiring phenological information across large geographic areas (Sakamoto, 2011).

Thompson and Paull (2017) used MODIS time series of the Normalized Difference Infrared Index (NDII = Band 2 - Band 6/Band $2+$ Band 6, where bands 2 and 6 are NIR and MIR bands, respectively) and NDVI from 2000 to 2014 to analyze land surface phenology for a high altitude area in the Australian Alps. For medium to lower elevation areas, the trend was towards a longer growing period (earlier start of season (SOS) and later end of season (EOS)); in this study elevation was a key factor influencing land surface phenology.

Wang et al. (2016) used time series of Global Inventory Modeling and Mapping Studies (GIMMS) AVHRR NDVI (cloud free, atmospherically and radiometrically corrected 15-day composite of AVHRR NDVI) and MODIS NDVI from 1982 to 2012 to investigate the spatial variability and temporal trends of vegetation phenology shifts in the northern hemisphere and compared the trends between three decades of study (1982-1992, 1992-2002 and 2002-2012). They found that the growing season duration in higher latitudes had increased, mainly due to an 
earlier SOS. The rate of advancement in SOS showed a stronger trend during the last decade of study (2002-2012).

Davis et al. (2017) studied long-term changes in vegetation phenology and productivity across the Namaqualand desert in northwest of South Africa using time series of AVHRR NDVI from 1982 to 2011 . The results showed that vegetation patterns vary along the gradients of rainfall. In addition, an earlier SOS, later EOS, and hence longer growing seasons were detected from 1982 to 2011. Increases in temperature, changes in land use practices and an increase in the concentration of atmospheric $\mathrm{CO}_{2}$ are suggested as some of the possible reasons for the shift in phenology.

Park et al. (2016) used time series of AVHRR NDVI and MODIS NDVI from 1982 to 2014 to investigate the spatiotemporal patterns and trend of changes in the growing season duration and productivity of boreal and arctic vegetation, and to quantify changes at a continental scale across the Northern Hemisphere (Eurasia and North America). They found an earlier SOS and later EOS across the study area during 2000-2014 while the opposite was found for 19822000. Vegetation productivity (growing season NDVI magnitude) also showed a significant increase during 1982-2014. A greater rate of change in the growing season and productivity was observed in Eurasia and the boreal extent through Eurasia and North America compared to the rest of North America and the arctic regions.

In an agricultural context, measuring phenological changes in vegetation is essential for estimating net primary production, crop yield monitoring, identification of regional crop dynamics and crop management (Zeng et al., 2016; Sakamoto et al., 2005). Identifying the phenological stages of crops provides information for important agricultural decision making such as irrigation scheduling and fertilizer management (Sakamoto et al., 2010). Vegetation indices (such as NDVI and EVI) derived from time series of AVHRR and MODIS have widely 
been used as indicators of crop productivity for measuring phenological changes in crops. For example, Zeng et al. (2016) used the Wide Dynamic Range Vegetation Index (WDRVI $=\alpha$ NIR$\mathrm{Red} / \alpha \mathrm{NIR}+\mathrm{Red}$, where $\alpha$ is a weighting coefficient) derived from MODIS time series to detect the critical vegetative and reproductive stages of corn and soybeans in Nebraska and Iowa, USA from 2009 to 2012. The results showed an abnormally late SOS for both corn and soybean in 2009 due to wet and cool spring weather and an earlier SOS for both corn and soybeans in 2012 due to an abnormally warm late-winter. Sakamoto et al. (2005) used MODIS EVI acquired in 2002 to estimate the phenological characteristics of paddy rice in Japan. They determined the planting date, heading date, harvesting date, and growing period in 2002. Skakun et al. (2017) used MODIS NDVI time series to accurately discriminate winter crops from spring and summer crops by measuring crop phenological metrics in Kansas and Ukraine. They calculated the phenological metrics of winter crops develop biomass during the time period before spring and summer crops to estimate the proportion of winter crops, derived the temporal profile of NDVI for spring and summer crops, and compared it to that of the winter crops to determine if the temporal profiles for these two crop classes could be used to discriminate them. This study showed that phenological metrics could be used to increase the accuracy of crop classification especially winter crop discrimination.

In addition, phenological metrics derived from time series of high temporal resolution imagery have been used as indicators of biodiversity in different landscapes. Phenological metrics that represent the seasonal variability of canopy spectral characteristics have been used to assess the spatial and temporal changes in the local distribution of individual species and regional biodiversity pools (Tuanmu et al., 2010). For example, Vina et al. (2012) studied the relationship between floral diversity and land surface phenology in Qinling Mountains in China using MODIS time series. They measured land surface phenology by temporal analysis of four vegetation 
indices, including the WDRVI, the Visible Atmospherically Resistant Index (VARI = (Green Red) / (Green + Red - Blue)), NDVI and EVI. They found that floristically similar vegetation types had similar phenology as detected by all four vegetation indices. This study showed that the spatial and temporal variability in phenological metrics can be used as predictors of plant diversity. Hurlbert and Haskel (2003) used AVHRR NDVI time series to assess the relationship between seasonality and avian species diversity. They found that metrics of seasonality derived from NDVI are good predictors of avian species diversity. Besides such studies, the literature on use of phenological metrics as predictors of local biodiversity, especially for modeling biodiversity-landscape heterogeneity relationships is still limited. This study hypothesizes that farmland biodiversity is related to the spatial and temporal variability in vegetation phenology at the landscape scale.

\subsection{Research objective and questions}

The objective of this study was to determine if vegetation phenological characteristics and temporal variability are related to local farmland biodiversity. The research questions addressed are listed as below:

a. Is there a relationship between vegetation phenology and local farmland biodiversity when phenology and biodiversity are measured coincidentally (in the same year)?

b. Does the strength of the vegetation phenology-biodiversity relationship vary when phenology is measured in past years? If so, is there a pattern showing a lag effect - i.e. phenology in previous years is more strongly related to biodiversity in the present year?

c. Is the crop phenology-biodiversity relationship stronger for larger $(3 \times 3 \mathrm{~km})$ than smaller $(1$ x $1 \mathrm{~km}$ ) landscape spatial extents? 
d. Of the seven taxa assessed, do certain taxa show consistently stronger biodiversity relationships with crop phenology?

\subsection{Data}

The study area, landscapes and biodiversity data were as described in Chapter 3.1 and 3.2.1. MODIS weekly Maximum-NDVI data provided by the Canadian Ag-Land Monitoring System (CALMS) of Agriculture and Agri-Food Canada (AAFC) throughout the growing season (spring/summer to early fall i.e. harvest season) were utilized in this study. A pixel size of $250 \mathrm{~m}$ and the time series was available from 2000. This product is generated in multiple steps, including

data calibration, cloud and snow removal and weekly composite creation. These steps, as well as the product specifications are described in detail in Appendix II.

\subsection{Methods:}

Figure 4.1. outlines the main processes and methods used in this chapter. A detailed description of each step follows. 


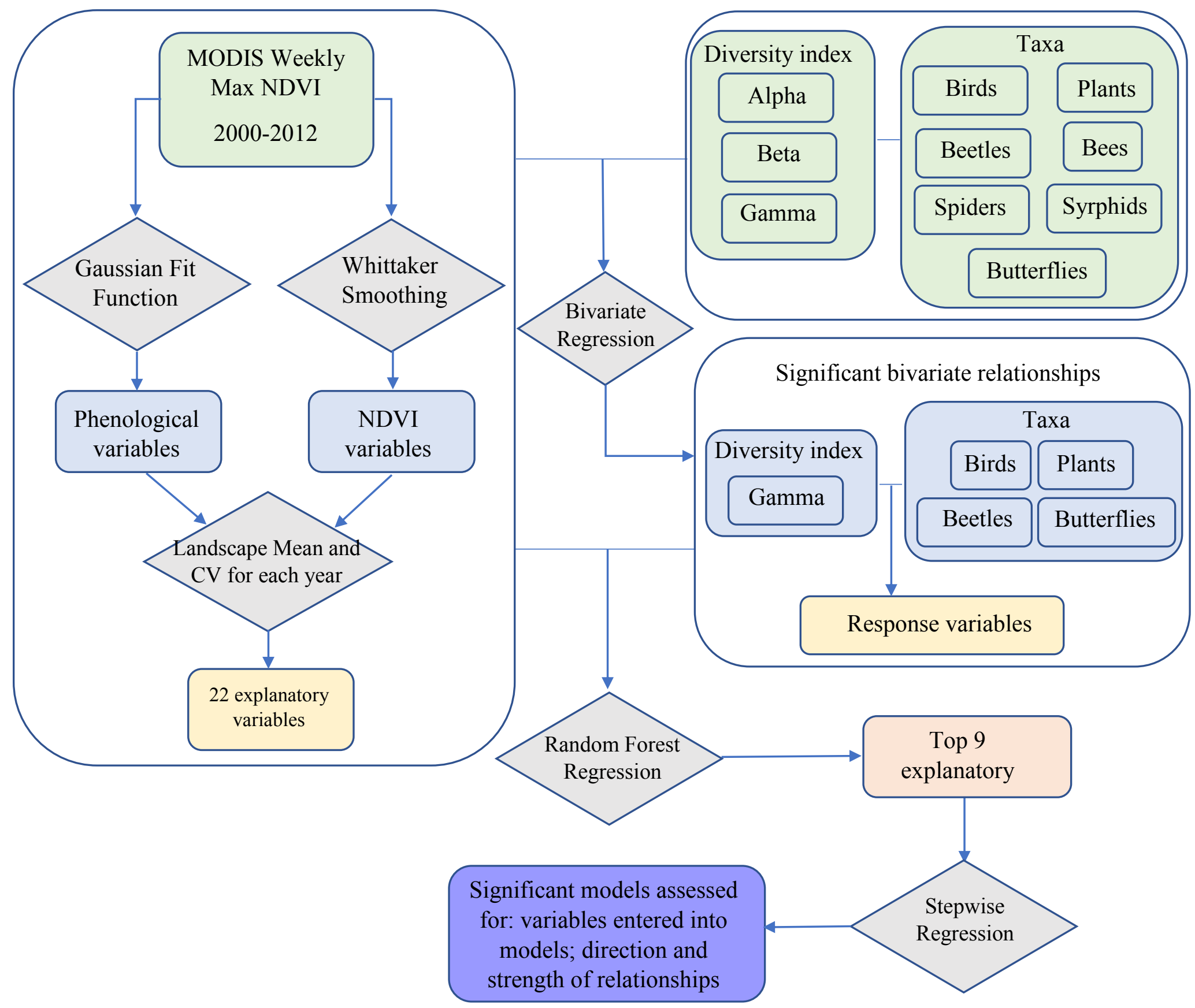

Figure 4. 1. Flow chart outlining various processing and analytical steps of this study. This includes the extraction of phenological variables from MODIS weekly Max NDVI and selection of biodiversity response variables and taxa, as well as the statistical modeling to assess the most important image variables and the direction and magnitude of their relationships with biodiversity. 


\subsubsection{Calculating phenological variables}

Plotting time series of NDVI produces a temporal curve that can be used to extract key phenological variables such as onset of greenness, peak and offset of development (McCloy and Lucht, 2004). Various algorithms have been used to derive variables related to vegetation productivity and phenology (Reed et al. 1994; Pettorelli et al., 2005; De Beurs and Henebry, 2010). They can be categorized in two groups: 1) algorithms that model the entire time series using a mathematical function, and 2) algorithms that estimate the timing of single phenological dates and variables, such as the start of the growing season (Beck et al., 2006).

In the first group, four methods have been used: thresholds, derivatives, smoothing, and model fitting (De Beurs and Henebry, 2010). Threshold methods are the simplest and most frequently applied to determine the SOS and EOS as the day of year when NDVI crosses a given threshold in an upward or downward direction, respectively (De Beurs and Henebry, 2010). Derivative methods extract the maximal increase and decrease in NDVI from the NDVI curves as SOS and EOS, respectively. SOS is characterized by the time of greatest leaf expansion or the fastest gradient in green-up during the growing season, whereas EOS corresponds to the date of the most negative derivative (Tateishi and Ebata, 2004). Smoothing function methods include Principal Component Analysis (PCA, which accounts for a maximum portion of variance present in the entire dataset through a linear combination of the original observations) (Hirosawa et al., 1996), Fourier analysis (which approximates complex curves with a sum of sinusoidal waves at multiple frequencies) (Azzali and Menenti, 2000; Jakubauskas et al., 2001; Stockli and Vidale, 2004, Silva et al., 2014), and Autoregressive Moving Average (ARMA) processing (Anyambe and Eastman, 1996; Sakamoto et al., 2005). These methods determine SOS and EOS as the dates that a smoothed time series crosses a curve established from moving average models (Reed et al., 1994). Model fitting methods including logistic models and the Gaussian fit function (Jönsson 
and Eklundh, 2002) have been developed to be easily adjusted to the available satellite data to allow for the variable nature of the NDVI curves (De Beurs and Henebry, 2010). Logistic models parametrize the NDVI temporal profile and divide it at the peak into two time series to estimate each part of the curve.

A Gaussian fit function, a moving average model and an adapted autoregressive moving average model (Reed et al., 1994) were compared by AAFC prior to this study with MODIS data for the whole agricultural extent of Canada. The extracted SOS and EOS outputs of each model were cross-referenced with ground measurements of crop emergence and harvest dates. The Gaussian fit function produced SOS and EOS dates that were closest to the ground measurements, hence, in this study, it was selected to extract phenological variables (Champagne 2017, pers. Comm). Gaussian fit function consists of a number of local model functions that are merged into a global function. The existing and merging of multiple local functions enhances the flexibility of the fittings and allows the overall fitted function to follow the complex behavior of the time series, which is not possible with a simple Gaussian model (Jönsson and Eklundh 2002, De Beurs and Henebry, 2010). The Gaussian-type local functions are as follows:

$$
\mathrm{NDVI}=\mathrm{C} 1+\mathrm{C} 2\left\{\begin{array}{l}
\exp \left[-\left(\frac{t-a_{1}}{a_{2}}\right)^{a_{3}}\right] \text { if } \mathrm{t}>\mathrm{a}_{1} \\
\exp \left[-\left(\frac{a_{1}-t}{a_{4}}\right)^{a_{5}}\right] \text { if } \mathrm{t}<\mathrm{a}_{1}
\end{array}\right.
$$

Where $\mathrm{C}_{1}$ and $\mathrm{C}_{2}$ determine the intercept and the amplitude of the curves, respectively, and $a_{1}$ determines the timing of the maximum (measured in time units). The upper part of the equation is fitted to the right half of the time series (time is after the peak $a_{1}$ is reached), while the lower part of the equation fits to the left half of the time series. The parameters $\mathrm{a}_{2}$ and $\mathrm{a}_{4}$ determine the width of the curves on the right and left side, respectively. The parameters a $a_{3}$ and a5 determine the flatness (or kurtosis) of the curves on the right and left side, respectively (De Beurs and 
Henebry, 2010). After this function has been fitted to the NDVI time series, SOS is determined from the global model as the day of year (DOY) at which the NDVI value reaches $40 \%$ of the difference between maximum NDVI and the first NDVI observation before greening starts. Similarly, EOS is calculated as the DOY at which the NDVI value reaches $40 \%$ of the difference between maximum NDVI and the last NDVI observation (when the curve flattens after full senescence or harvest, Figure 4.2).

The phenological variables that were extracted from the Gaussian fit model in this study are listed below. These metrics are defined by United States Geological Survey (USGS) as follows (https://phenology.cr.usgs.gov/methods_metrics.php):

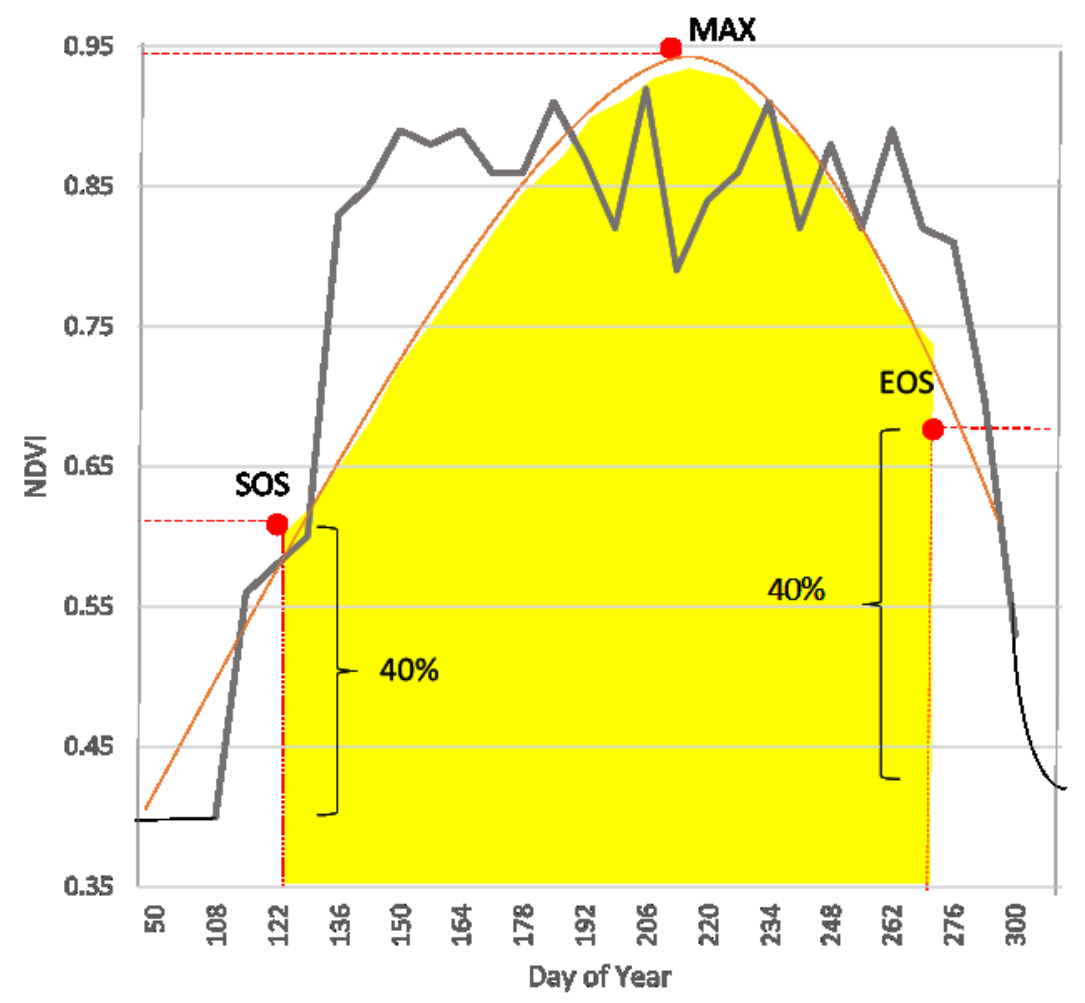

Figure 4. 2. The Gaussian fit function calculates SOS as $40 \%$ of the difference between first NDVI observation and maximum NDVI and EOS as $40 \%$ of the difference between maximum NDVI and the last NDVI observation. 
- Start of Season Day of Year (SOS DOY): Beginning of measurable photosynthesis in the vegetation canopy. Day of year identified as having a consistent upward trend in time series of NDVI.

- Start of Season NDVI (SOS NDVI): NDVI value (or baseline) at the beginning of measurable photosynthesis, i.e. start of season day of year.

- End of Season Day of Year (EOS DOY): End of measurable photosynthesis in the vegetation canopy. Day of year identified at the end of a consistent downward trend in time series of NDVI.

- End of Season NDVI (EOS NDVI): NDVI value at the end of measurable photosynthesis which is corresponding to the end of season day of year.

- Time of maximum (MAX DOY): Day of year corresponding to the maximum NDVI or maximum photosynthesis in an annual time series.

- Maximum NDVI (MAX NDVI): Maximum NDVI value in an annual time series.

- Season Duration (SEAS DUR): Length of photosynthesis activity in the growing season, i.e. number of days from the SOS DOY to EOS DOY.

- Season Amplitude (SEAS AMP): Maximum increase in canopy photosynthesis activity above the baseline, i.e. the difference between MAX NDVI and SOS NDVI.

- Season Integrated (SEAS INT): Canopy photosynthesis activity or daily integration of NDVI above zero for the entire duration of the growing season by summing the NDVI values for all days between SOS and EOS; generally represents net primary productivity.

- Season Greenness (SEAS GRE): Canopy photosynthesis activity or an integration of the area under the NDVI curve above the baseline (a line connecting SOS to EOS) for the entire duration of the growing season. 


\subsubsection{Whittaker smoothing algorithm}

One of the main challenges in time-series analysis of remote sensing data is dealing with image noise. The noise is usually introduced by the sensor or caused by residual atmospheric effects such cloud, shadow, atmospheric dust and aerosols (Atkinson et al., 2012; Shao et al., 2016). In addition, in medium and coarse spatial resolution data different land cover types within a pixel can introduce image noise (Atkinson et al., 2012). Such outliers can create anomalies in the temporal profile of a remote sensing time series, therefore it is necessary to clean the data and apply smoothing filters (Holben, 1986; Lunetta et al., 2006). A large number of data smoothing algorithms exist for that purpose. Wavelets (a small localized wave in time or space which is used to remove noise) and Fourier transforms (as the sum of a series of cosine waves and a constant term) (Sakamoto et al.,2005), the Savitzky-Golay filter (which applies a moving window with a user defined width to fit all points in the time series and reduce noise) (Chen et al.,2004), and the Whittaker smoothing algorithm (Atzberger and Eilers,2011a) are examples that have been applied on MODIS NDVI time series (Shao et al., 2016).

The Whittaker algorithm has been made popular in remote sensing time series application by Eilers (2003) and has been successful in enhancing NDVI temporal profile smoothness and signal-to-noise ratio (Atzberger and Eilers, 2011; Shao et al., 2016). In addition, this filter smooths the data directly without any pre-processing. It is extremely fast, gives continuous control over smoothness with only one parameter and interpolates automatically. The Whittaker smoothing filter had been shown to outperform other algorithms (Atzberger and Eilers 2011; Atkinson et al., 2012; Shao et al., 2016), hence it was implemented in this study to produce a smoothed NDVI temporal profile. 
The Whittaker algorithm is based on penalized least squares; it fits a series to discrete data and penalizes the roughness of the new smoothed curve. In this way, it balances reliability of the data and roughness of the fitted data. The smoother the result, the more it will deviate from the input data. A balanced combination of the two goals is the sum $(Q)$ :

$$
Q=S+\lambda \mathrm{R}
$$

(Equation 4.2)

Where

$$
\begin{gathered}
S=\sum_{t}\left(V I(t)-V I^{*}(t)\right)^{2} \\
R=\sum_{t}\left(V I^{*}(t)-3 V I^{*}(t-1)+3 V I^{*}(t-2)-3 V I^{*}(t-3)\right)^{2}
\end{gathered}
$$

Where VI is the vegetation index value from the data, VI* is the smoothed vegetation index, $S$ is measured as the sum of squared differences between the original VI data and the smoothed VI data, and $\mathrm{R}$ is the roughness of the smoothed curve and expressed here as third order differences. $\lambda$ is the smoothing parameter and chosen by the user. The aim of penalizing least squares is to find the smoothed series $\mathrm{VI}^{*}(t)$ that minimizes $Q$. The larger the parameter $\lambda$, the greater the influence of $R$ on $Q$ and the smoother will be $\mathrm{VI}^{*}(t)$ (Atzberger and Eilers, 2011; Atkinson et al. 2012).

\subsubsection{Extracting phenological variables for sample landscapes}

The spatial mean and coefficient of variation $(\mathrm{CV}=\mathrm{Std}$. deviation/mean $)$ for all phenological variables, as well as NDVI variables derived from smoothed temporal profiles were extracted for all sample landscapes independently for each year from 2000 to 2012 . Use of CV allows a standardization of variability of NDVI across landscapes with different degrees of agricultural cover and crop types. In addition, it is unit-less and enables comparison of predictors with 
different measurement scales (Duro et al., 2014). The mean of the phenological variables represents the spatial average of that variable across all pixels within each sample landscape. For example, the mean of SOS DOY shows the spatial average over the sample landscape of the time of emergence for different crops and vegetation covers, and the mean of SOS NDVI shows the spatial average of NDVI values at the time of emergence over the landscape. The average NDVI for each landscape over the growing season was extracted from the smoothed temporal profile of each year. In this context, mean NDVI is representative of annual average of crop productivity. Furthermore, CV represents the spatial variability of the metrics across each sample landscape. For example, CV of SOS DOY represents the variability amongst the pixels in a landscape of the timing of emergence between different crops and vegetation covers and CV of SOS NDVI shows the variability amongst the landscape pixels in the NDVI values at the time of emergence. CV of average growing season NDVI represents the variability in average growing season vegetation productivity across the pixels of each sample landscape and is a simple measure of spectral variability or image-texture. CV has been used in this manner as a proxy for spatial variability of land cover and as an indirect indicator of potential biological diversity (Rocchini et al., 2010; Duro et al., 2014). It is a standardized statistical coefficient that allows comparison between sample landscapes with various levels of crop productivity, as well as comparison of different explanatory variables with different measurement scales.

\subsubsection{Statistical modeling}

\subsubsection{Exploratory modeling for selecting response variables}

Initial exploratory modeling was conducted using bi-variate regression models to portray the relationship between alpha, beta and gamma diversity of the seven taxa groups and the MODISbased phenological variables. The goal was to determine if any of the biodiversity metrics for any of the taxa demonstrate significant relationships $(\mathrm{p} \leq 0.05)$ with the phenological variables. The 
biodiversity index showing the strongest relationships was used as the response variable in multivariate random forest regression (RFR) and step-wise regression (SWR) models. Variables that did not meet these criteria were eliminated from further analysis.

Bi-variate regression was also run between the alpha, beta, and gamma diversity of the selected taxa and the percentage of agriculture $(\% \mathrm{Ag})$ in each sample landscape to test the effect of nonagricultural land covers on species diversity. Percent Ag was calculated using the land cover maps derived from aerial photos of 2011 and 2012. This relationship was tested prior to this study by Duro et al. (2014) for 2011 (45 sample landscapes) and Fahrig et al. (2015) for 2011 and 2012 sample landscapes at $1 \times 1 \mathrm{~km}$ extent. In this study, this relationship was analyzed for all landscapes (2011 and 2012) and compared for $1 \times 1 \mathrm{~km}$ and $3 \times 3 \mathrm{~km}$ extents.

Overall, 22 explanatory variables were used to create biodiversity models. To test the relative effect of sample landscape size on the models, all explanatory variables were extracted for $1 \times 1 \mathrm{~km}$ (the extent at which biodiversity were surveyed) and $3 \times 3 \mathrm{~km}$ (the maximum possible extent identified by Pasher et al. (2011) for the landscape heterogeneity metrics) sample landscapes independently.

\subsubsection{Random forest regression}

Random forest regression (RFR) was used as a means to determine relative variable importance in models of biodiversity. Random forests (RF; Breiman, 2001) is a non-parametric decision tree algorithm for regression (as in this research) or classification that is based on aggregation of the results of many iteratively produced decision tree models. It is an ensemble learning method, appearing in the machine learning literature at the end of 1990s (Genuer et al., 2010). RF incorporates bagging, where, for each tree that is iteratively created a random bootstrap sample of the reference data is selected for model development and the remaining data are used for model validation. Following model creation, the data are returned to the reference dataset and another 
random sample is selected for the subsequent tree; this process is repeated for a user-specified number of trees. The percent variance explained and the mean of squared residuals are measured as out-of-bag error. These errors are calculated using a user specified proportion of the reference samples for each tree (1/3 was the default and used in this study). The percent variance value characterises the proportion of the variance explained by the validation sample and the mean of squared residuals is indicative of the difference between the observed and predicted values (Breiman, 2011).

Random forest also selects at each node a random subset of explanatory variables in order to reduce potential correlation between trees that may occur if all variables were used and some or all are correlated (Liaw and Wiener, 2002; Genuer et al., 2010). At each node, the best split is calculated using only this variable subset (user defined number of variables). As opposed to classification and regression tree $(\mathrm{CaRT})$ methods, pruning of trees is not performed in random forest so all the trees of the forest are maximally grown (Genuer et al., 2010). In the end, following creation of the specified number of trees, the mean value of the predictions for the dependent variable is selected (Liaw and Wiener, 2002).

RFR is an effective, accurate and user-friendly tool in prediction that outperforms most regression algorithms (Gromping, 2009). It creates a strong framework in which the individual predictors and their correlations indicate its ability to predict. It is not sensitive to large numbers of input variables and is robust to overfitting (Breiman, 2001). In addition, RFR is capable of estimating variable importance by calculating the increase in prediction error when out-of-bag data for that variable are randomly changed (permuted) while all others are left unchanged. The calculations are carried out tree by tree as the random forest is constructed (Liaw and Wiener, 2002). 
RF has become popular for satellite image classification and has been widely used for measuring relative importance of variables in image classification (Millard and Richardson, 2015; Lindsay et al., 2019; Olimb and Robinson, 2019). RFR was used by Fairbrother (2016) prior to this research for variable selection in regression models with large number of thematic map derived variables for the same sample landscapes as this research. RF was selected in this thesis for experimental purposes, to follow up on that work, and to test the utility of such machine learning techniques in variable selection for regression models.

Although RF may produce models with non-linear relations between predictor and dependent variables, scatterplots of all bivariate relationships between biodiversity and each dependent variable showed high scatter and no discernable non-linear relationships. RFR was run in the randomForest R program based on a script written by Fairbrother (2016), adapted from Millard and Richardson (2015). This script runs with 100 iterations and each iteration produced 1000 trees; Millard and Richardson (2015) found this to be the optimum number of iterations and trees for their wetland remote sensing data in the same general region (eastern Ontario) and it was expected to be appropriate for the data in this research. The script produces a list of explanatory variables with their associated percent Increase in Mean Squared Error (\%IncMSE) derived from the random permutation process. \%IncMSE can be positive and negative and it is higher for more important variables (Genuer et al., 2010). Based on the values of \%IncMSE from 100 iterations in each individual year, the explanatory variables were ranked and the top three most important variables for each iteration were identified. The number of times in the 100 iterations that each variable was one of the top three most important variables was then calculated. The frequency of occurence of each variable in the top three was summed up over all 13 years and the variables were then ranked; i.e. the ranking was based on the number of occurences in the top three over 
all iterations and years $(100$ iterations $* 13$ years $=1300$ maximum frequency $)$. This process was conducted for $1 \times 1 \mathrm{~km}$ and $3 \times 3 \mathrm{~km}$ sample landscapes independently.

The equivalent of out-of-bag error for RFR is comprised of the percent variance explained (\% Var) and the mean of squared residuals (MSR) for one third of the samples of each RFR run. The percent variance explained (\% Var) is calculated as follows (Matsuki et al. 2016):

$$
\% \operatorname{Var}=\quad x=1-\frac{\sum_{i=1}^{n}\left(\hat{y}-y_{i}\right)^{2}}{\sum_{i=1}^{n}\left(\bar{y}-y_{i}\right)^{2}}
$$

$\%$ Var is a pseudo $\mathrm{R}^{2}$ equal to [1 - mean sum of squared error / variance of $\mathrm{y}$ variable] (where the y variable is biodiversity). It could be negative if the mean sum squared error in the out-of-bag prediction is greater than the variance of the training set. A negative $\%$ Var shows that the out-of-bag predictions do not explain the variance of the training set well and this variance was larger than the total variance or the variance of the remaining $2 / 3$ of the data. It indicates poor ability of models for predicting the response variable. (Liaw and Wiener, 2002).

\subsubsection{Step-wise multiple linear regression}

The most important variables in multiple RFR runs were then used as input to step-wise regression (SWR). The goal was to determine which phenological variables formed significant models with biodiversity and the direction (+/-) of those relationships; i.e., it was not to develop models for prediction of biodiversity. SWR was selected for the final modelling stage because it provides easily interpretable and conventional statistical model performance indicators. Also, as stated above for RFR, no distinct non-linear trends in biodiversity-phenology scatterplots were evident that would favour a non-linear model over a linear model, and even if some non-linear relationships were present, their direction (+/-) would be the same as for linear relationships. 
SWR is a method in which explanatory variable selection is performed by an automatic procedure. In each step, a predefined criterion is used to add or subtract a explanatory variable to or from the set of explanatory variables. Forward SWR was used in this study. It begins with no candidate variables in the model and iteratively selects the variable that accounts for the most remaining variance (highest $\mathrm{R}^{2}$ ) in the dependent variable. This iterative procedure continues until none of the remaining variables accounts for a significant amount of the residual variance. After adding each variable, all candidate variables are checked to see if their significance has been reduced below the threshold and if a variable is found to be non-significant, it is removed from the model. A significance criterion of $\mathrm{p} \leq 0.05$ was used for any variable to be entered into or removed from the model. To limit the number of potential model variables, a 10:1 (observations: variables) ratio was used (Harrell et al., 1984). Given the number of sample landscapes was 93, the top 9 phenological variables identified in RFR were used as input in the SWR.

SWR was run in olsrr package in R for gamma diversity (selected as the best biodiversity index) of each taxon using the $1 \times 1 \mathrm{~km}$ and $3 \times 3 \mathrm{~km}$ landscape metrics independently to determine model significance, the explanatory variables entered, and the direction of their relationship with gamma diversity. Linearity of all input variables with gamma diversity, the variance inflation factor (O'brien, 2007), and the normality and uniformity of the model residuals were checked and no violations of these criteria were found. The absolute value of the overall $r$ of the models was used to compare the models' performance while the magnitude and direction of the $r$ value of individual variables which entered the models were compared with a $95 \%$ confidence interval to find the most important variable for each taxon. Confidence intervals were calculated following Lane et al. (2007). In this method $r$ values were first transformed to Fisher's $\mathrm{z}^{\prime}$ to normalize the distribution of $\mathrm{r}$ and minimize skewness. Upper and lower limits were calculated using Equation 4.6: 
Lower limit $=-\mathrm{z}^{\prime}-(1.96) \times($ standard error $)$

Upper limit $=-\mathrm{z}^{\prime}-(1.96) \times($ standard error $)$

(Equation 4.6)

Where standard error $=\frac{1}{\sqrt{\mathrm{N}-3}}$ and $N=$ sample number (93)

\subsubsection{Alternative statistical methods}

Random Forest has been shown in some cases to be biased when input variables are correlated, or when the remote sensing data have high dimensionality and the training sample size is small (Millard and Richardson, 2015; Belgiu and Dragut, 2016; Gromping, 2009). The threshold for identifying high between-variable correlation is arbitrary and different r-values have been used as the threshold in different sources. For example, Behnamian et al. (in press) indicated $r<0.85$ as the threshold for high between-variable correlation. In this study, the highest correlation between two input variables was $r=0.64$ and the average was $r=0.38$. In addition, Behnamian et al. (in press) demonstrated that RF classification would not suffer from high among-variable correlation bias if the number of variables is higher than 4 . In this study, 22 variables were used in RFR modeling which would resolve the concerns regarding among-variable correlation. Nonetheless, due to such potential limitations of RFR it was decided to test two alternative statitstical variable selection techniques to determine if the selected model variables were consistent between techniques.

These tests were conducted for a subset of the data $(3 \times 3 \mathrm{~km}$ extent; plants and beetles; years 2000, 2006 and 2011) and the results were compared to those of RFR. Corrected Akaike's Information Criterion (AICc, Burnham and Anderson, 2002) and the method proposed by Lindeman, Merenda and Gold (LMG, Gromping et al., 2006) were the selected alternative statistical methods. 
AICc was selected because it has been widely used in biostatistics for determining the relative importance of explanatory variables and the direction and magnitude of relations between the predictors and the response variables (Duro et al., 2014; Fahrig et al., 2015; Wilson et al., 2017). AICc is corrected AIC and calculated using Equation 4.7:

$\mathrm{AICc}=\mathrm{AIC}+\frac{2 \mathrm{k}(\mathrm{k}+1)}{\mathrm{n}-\mathrm{k}-1}$

(Equation 4.7)

Where $\mathrm{k}$ is the number of parameters and $\mathrm{n}$ is the sample size. AIC is calculated using Equation 4.8:

$A I C=2 k-2 \ln (L)$

(Equation 4.8)

Where $\mathrm{L}$ is the maximum likelihood value and is a measure of model fit. Higher likelihood numbers indicate better model fit.

AICc was run using the MumIn package and AICc function in R (Barton, 2002). It was run in two steps. First, AICc was run for all 22 single variable models and the top six models with lowest AICc were selected. In the second step, the top six variables were added into the model by their order of importance in a forward stepwise manner and subsequently variables were removed from the model by their order of importance (most important variables were removed first) in a backward stepwise manner to test the effect of the most important variables on the models. AICc was calculated for the forward and backward approach independently.

LMG was selected because it assesses variable relative importance in linear regression while addressing the issue of among-variable correlation. Relative importance is defined as the proportion that each explanatory variable contributes to the overall model $\mathrm{R}^{2}$ both directly and in combination with other variables (Johnson and Lebreton, 2004). LMG was run in in R using the relaimpo package and the calc.relimp function (Gromping, 2006). All 22 variables were input. 
The output was the overall $\mathrm{R}^{2}$ of the model, as well as the proportion of $\mathrm{R}^{2}$ in which each explanatory variable contributes to the model.

\subsubsection{Evaluating potential lag effect}

To test the potential time lagged response of taxa to phenological conditions of the past, the most significant variable(s) which entered the SWR models of each taxon most frequently across all years were selected as the explanatory variable. The selected variable for 2012 was first modeled against the diversity index, then the same variable for previous years was added to that of 2012 one year at a time in a step-wise manner (Table 4.2) and modeled against the biodiversity index using a linear regression model. The Pearson $\mathrm{R}$ for all the models was compared to determine if adding the given variable from past years' data would affect model performance. An increase in the $\mathrm{R}$ value when a variable from previous years was added would indicate a time lagged response of the taxon to temporal changes in vegetation phenology.

Table 4. 1. List of models with best explanatory variable of each year to test time lagged response of species to phenological changes

\begin{tabular}{|c|l|}
\hline Models & Input explanatory variables \\
\hline M1 & $V_{2012}$ \\
\hline M2 & $V_{2012}+V_{2011}$ \\
\hline M3 & $V_{2012}+V_{2011}+V_{2010}$ \\
\hline M 4 & $V_{2012}+V_{2011}+V_{2010}+V_{2009}$ \\
\hline M5 & $V_{2012}+V_{2011}+V_{2010}+V_{2009}+V_{2008}$ \\
\hline M6 & $V_{2012}+V_{2011}+V_{2010}+V_{2009}+V_{2008}+V_{2007}$ \\
\hline M7 & $V_{2012}+V_{2011}+V_{2010}+V_{2009}+V_{2008}+V_{2007}+V_{2006}$ \\
\hline M8 & $V_{2012}+V_{2011}+V_{2010}+V_{2009}+V_{2008}+V_{2007}+V_{2006}+V_{2005}$ \\
\hline M9 & $V_{2012}+V_{2011}+V_{2010}+V_{2009}+V_{2008}+V_{2007}+V_{2006}+V_{2005}+V_{2004}$ \\
\hline M10 & $V_{2012}+V_{2011}+V_{2010}+V_{2009}+V_{2008}+V_{2007}+V_{2006}+V_{2005}+V_{2004}+V_{2003}$ \\
\hline M11 & $V_{2012}+V_{2011}+V_{2010}+V_{2009}+V_{2008}+V_{2007}+V_{2006}+V_{2005}+V_{2004}+V_{2003}+V_{2002}$ \\
\hline M12 & $V_{2012}+V_{2011}+V_{2010}+V_{2009}+V_{2008}+V_{2007}+V_{2006}+V_{2005}+V_{2004}+V_{2003}+V_{2002}+V_{2001}$ \\
\hline M13 & $V_{2012}+V_{2011}+V_{2010}+V_{2009}+V_{2008}+V_{2007}+V_{2006}+V_{2005}+V_{2004}+V_{2003}+V_{2002}+V_{2001}+V_{2000}$ \\
\hline
\end{tabular}




\subsection{Results:}

\subsubsection{Phenological variables}

The Gaussian fit function output was comprised of rasters with $250 \mathrm{~m}$ pixel size, each representing the corresponding phenological variable for each year. The range of pixel values varied depending on the variable represented. For those that represent day of year (SOS DOY, MAX DOY and EOS DOY) and SEAS DUR, the pixel values range between 1 and 365, while for those variables that represent NDVI (SOS NDVI, EOS NDVI, MAX NDVI and SEAS AMP) values the pixel values range between 0 and 1 . No values of NDVI below zero occurred in any of the landscapes. The pixel value range for SEAS INT and SEAS GRE varied in relation to the integration of the area under the phenology curve. Figures 4.3 and 4.4 are examples of the output maps produced for SOS DOY and SOS NDVI for the year 2000, respectively.

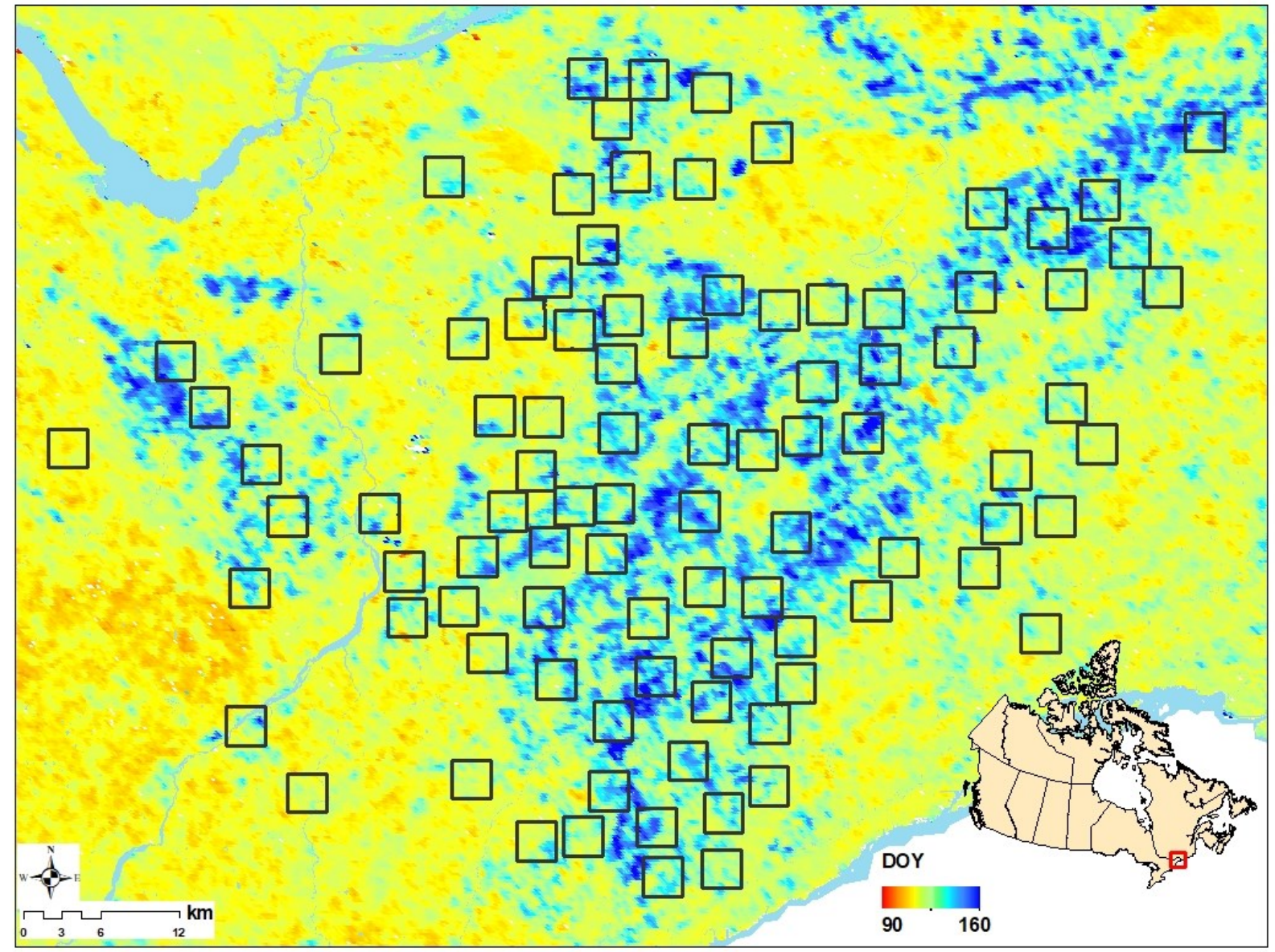

Figure 4. 3. Raster representing SOS DOY for year 2011. The sample landscapes are shown as black squares. 


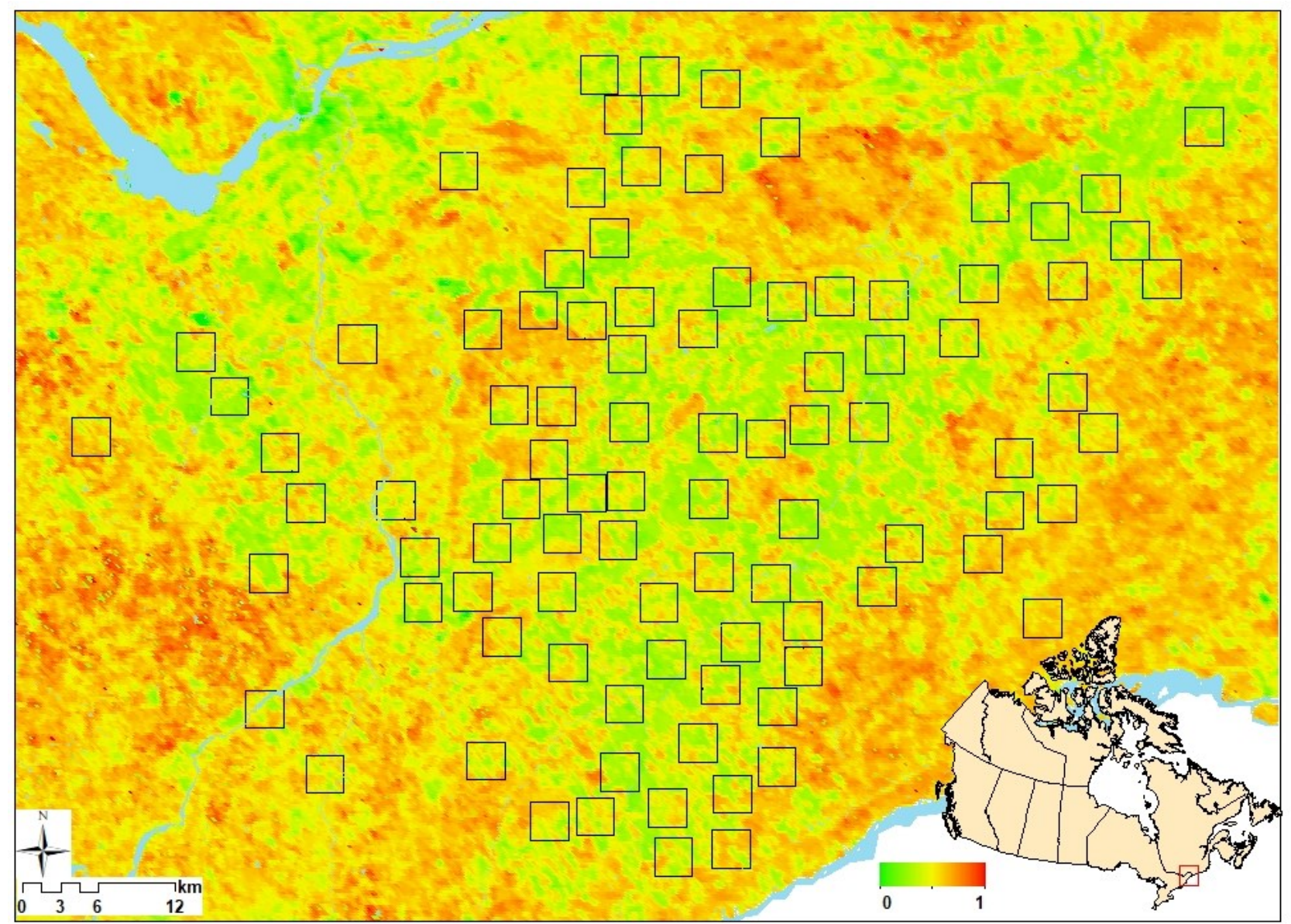

Figure 4.4. Raster representing SOS NDVI for year 2011. The sample landscapes are shown as black squares.

\subsubsection{Statistical modeling}

\subsubsection{Exploratory modeling for selecting response variables}

Results of exploratory bivariate regression modeling of phenological variables versus alpha, beta and gamma diversity for all taxa showed that plants $(0.01 \leq|\mathrm{r}| \leq 0.40)$ and beetles $(0.01 \leq|\mathrm{r}| \leq$ 0.50)_demonstrated the strongest relationships in most years; they were therefore selected for RFR modelling. Syrphids $(0.01 \leq|\mathrm{r}| \leq 0.21)$ and spiders $(0.01 \leq|\mathrm{r}| \leq 0.20)$ did not demonstrate significant relationships with the phenological variables and were eliminated from further modelling. Bees $(0.01 \leq|\mathrm{r}| \leq 0.30)$ and butterflies $(0.01 \leq|\mathrm{r}| \leq 0.31)$ demonstrated similar strength 
of relationships with the phenological variables in most years, however, bees were only surveyed in 2011, resulting in a smaller sample size than that of other taxa. Bees were therefore eliminated, and butterflies were selected for RFR modelling. Bird diversity model strength varied between the phenological variables and years. They were retained for RFR modelling due to their conservational value and importance in agroecosystems. In addition, bird diversity had been modeled in previous research by Duro et al. (2014), Fahrig et al. (2015) and Fairbrother (2016) using the same sample landscapes and field data but different spatial data and explanatory variables, thus they were selected to compare the results to those studies.

The relationships between alpha, beta and gamma diversity indices were tested prior to this study (Duro et al. 2014; Fahrig et al., 2015) and moderate to high positive correlations ( $\mathrm{r} \geq$ 0.58 ) were found for plants, beetles, butterflies and birds. Alpha and beta were least correlated, while gamma was highly correlated $(r>0.88)$ with both alpha and beta.

No statistically significant relationship $(\mathrm{p}>0.05)$ between alpha, beta and gamma diversity of plants, beetles, birds and butterflies and \% Ag was found by Duro et al. (2014) using half the landscapes as surveyed in 2011, nor by Fahrig et al., (2015) using all 1 x $1 \mathrm{~km}$ landscapes surveryed in 2011 and 2012. The same applied to $3 \times 3 \mathrm{~km}$ landscapes for these four taxa as tested in this study; although most relationships were negative (increasing agriculture in the landscape is associated with lower biodiversity), they were generally non-significant $(\mathrm{p}>0.05)$. Exceptions were beetle beta and plant alpha at $1 \times 1 \mathrm{~km}(\mathrm{r}=-0.32$ and -0.31 respectively $)$ and beetle gamma and beta at $3 \times 3 \mathrm{~km}(\mathrm{r}=-0.34$ and -0.40 , respectively). The relationships were weakest for birds and butterflies and strongest for beetles and plants (Table 4.3). These results demonstrated that the variation in $\% \mathrm{Ag}$ and other land cover types within the sample landscapes did not have a significant effect on the species diversity. Fahrig et al. (2015) also found a weak relationship between $\% \mathrm{Ag}$ and Shannon crop diversity $(\mathrm{r}=0.14)$ which supports these results. 
Table 4.2. Pearson $r$ values for correlations between the percentage agriculture land cover in each sample landscape at $1 \times 1 \mathrm{~km}$ and $3 \times 3 \mathrm{~km}$ extent, and each biodiversity variable for the four selected taxa. Significant $(\mathrm{p} \leq 0.05)$ relationships are shown in bold.

\begin{tabular}{|c|c|c|}
\hline & $1 \times 1 \mathrm{~km}$ & $3 \times 3 \mathrm{~km}$ \\
\hline Beetle Alpha & -0.16 & -0.21 \\
\hline Beetle Gamma & -0.27 & $\mathbf{- 0 . 3 4}$ \\
\hline Beetle Beta & $\mathbf{- 0 . 3 2}$ & $\mathbf{- 0 . 4 0}$ \\
\hline Plant Alpha & $\mathbf{- 0 . 3 1}$ & -0.23 \\
\hline Plant Gamma & -0.25 & -0.19 \\
\hline Plant Beta & -0.20 & -0.15 \\
\hline Butterfly Gamma & -0.10 & -0.05 \\
\hline Butterfly Alpha & -0.13 & -0.05 \\
\hline Butterfly Beta & -0.17 & -0.14 \\
\hline Bird Alpha & -0.12 & -0.13 \\
\hline Bird Gamma & -0.10 & -0.04 \\
\hline Bird Beta & -0.07 & 0.01 \\
\hline
\end{tabular}

In addition, the relationship between MODIS-based explanatory variables and \% Ag was tested and strong relationships were found, especially between \% Ag and Mean SOS DOY, Mean SOS NDVI, Mean SEAS DUR and Mean SEAS INT $(r=0.78, r=-0.78, r=-0.69$ and $r=-0.76$, respectively, Table 4.4). A strong positive relationship between Mean SOS DOY and \% Ag indicated that more agriculture in a given landscape is associated with a later start of the growing season. Mean SOS NDVI and Mean SEAS INT demonstrated a negative relationship with \% Ag, which indicated that an increase in the amount of cultivated land in a sample landscape was coupled with lower average vegetation productivity at the time of onset of greenness and over the growing season. The strong relationship between MODIS-based explanatory variables and \% Ag suggested that $\% \mathrm{Ag}$ was reflected and manifested in these explanatory variables. This, coupled with the weak relationships of $\% \mathrm{Ag}$ with the diversity indices, showed that $\% \mathrm{Ag}$ did not need to be considered as a explanatory variable in subsequent modeling. 
Table 4.3. R values between the $\% \mathrm{Ag}$ and $3 \times 3 \mathrm{~km}$ explanatory variables extracted from 2011 MODIS data. Significant $r$ values $(\mathrm{p} \leq 0.05)$ are in bold.

\begin{tabular}{|c|c|c|c|c|c|c|c|c|c|c|c|c|c|c|c|c|c|c|c|c|c|c|}
\hline & 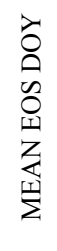 & $\begin{array}{l}\vec{z} \\
\grave{0} \\
0 \\
0 \\
0 \\
0\end{array}$ & 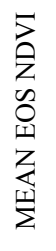 & $\begin{array}{l}\vec{z} \\
\text { 文 } \\
\text { Z } \\
0 \\
0 \\
0\end{array}$ & 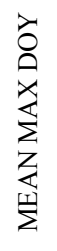 & 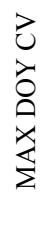 & 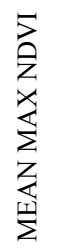 & 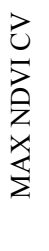 & 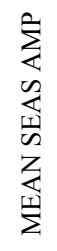 & 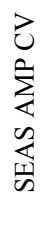 & 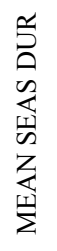 & 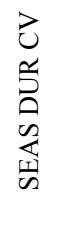 & 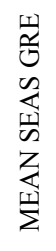 & 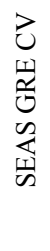 & 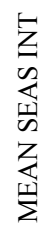 & 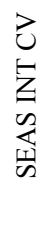 & 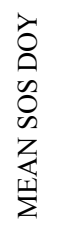 & $\begin{array}{l}\vec{z} \\
\grave{0} \\
\not \\
0 \\
0 \\
0\end{array}$ & 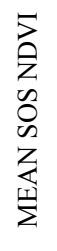 & 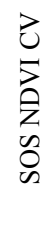 & 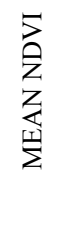 & $\begin{array}{l}\vec{z} \\
\text { z } \\
\text { z }\end{array}$ \\
\hline$\% \mathrm{Ag}$ & $\overrightarrow{\widetilde{\sigma}}$ & $\hat{n}$ & $\begin{array}{l}0 \\
i n \\
i\end{array}$ & $\stackrel{0}{0}$ & $\stackrel{\infty}{n}$ & $\begin{array}{l}\circ \\
0 \\
0\end{array}$ & $\begin{array}{l}0 \\
\text { ơ } \\
i \\
i\end{array}$ & $\begin{array}{l}\bar{i} \\
\end{array}$ & $\tilde{n}$ & $\frac{m}{0}$ & $\begin{array}{l}\text { ôे } \\
\text { i }\end{array}$ & $\stackrel{8}{\circ}$ & $\hat{n}$ & त्ञ̂ & $\begin{array}{l}\stackrel{0}{0} \\
\stackrel{i}{i}\end{array}$ & 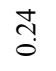 & $\stackrel{\infty}{\stackrel{\infty}{\circ}}$ & f. & $\underset{\substack{\infty \\
\stackrel{\infty}{i}}}{ }$ & $\stackrel{7}{0}$ & $\begin{array}{l}\hat{0} \\
\stackrel{1}{0}\end{array}$ & $\stackrel{0}{\circ}$ \\
\hline
\end{tabular}

Results of the above exploratory modelling between alpha, beta and gamma and explanatory variables varied across taxa and years but generally demonstrated poorer and insignificant model strength for alpha diversity. Beta and gamma varied in strength but both mostly showed significant relationships for all taxa in most years. Beta demonstrated a slightly stronger relationship in some models (examples presented in Figure 4.5). However, as mentioned above gamma is highly correlated with alpha and beta $(\mathrm{r}>0.88)$ and it represents the overall diversity and a broader range for all taxa compared to alpha and beta. Hence, it was deemed to be a better indicator of biodiversity for modelling against phenological metrics of coarse spatial resolution data. It was therefore selected as the response variable for RFR modelling. 

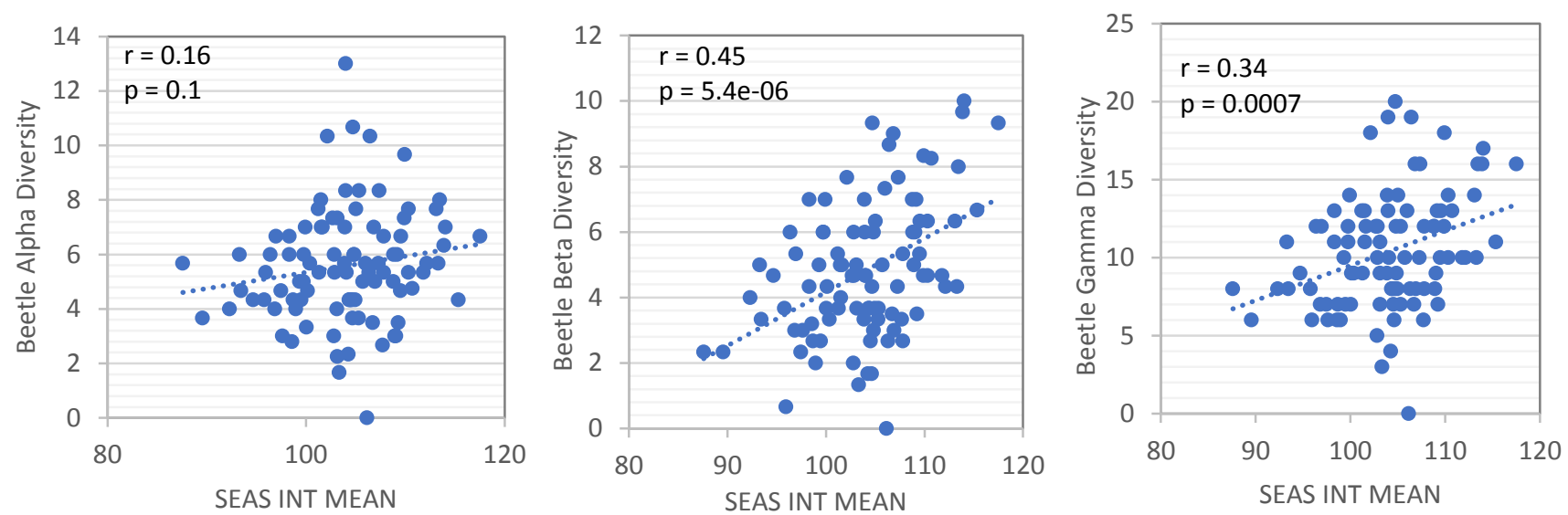

Figure 4.5. Comparing the relationship of alpha, beta and gamma diversity of beetles with Mean

SEAS INT in 2011. An example of poorer relationships found for alpha and stronger relationships for beta and gamma diversity.

Lastly, the correlation among the explanatory variables was tested for each year independently. All variables generally displayed low to moderate positive or negative correlation. Mean SOS NDVI and Mean EOS DOY showed the lowest correlation $(\mathrm{r}=-0.015)$ while Mean SOS NDVI and Mean SEAS INT showed the highest correlation $(r=0.64)$; the average $r$-value was 0.38 . Pearson correlation matrices for all explanatory variables for the $3 \times 3 \mathrm{~km}$ sample landscapes in the years 2011 and 2012 are presented in Appendix III. Correlations between explanatory variables for $1 \times 1 \mathrm{~km}$ landscapes were generally higher than those for $3 \times 3 \mathrm{~km}$.

\subsubsection{Random forest biodiversity modelling}

Random Forest Regression was run for the selected taxa using MODIS phenological and NDVI indices derived for each year (2000-2012) as the predictors, and gamma diversity from 2011 and 2012 (46 and 47 landscapes measured in 2011 and 2012, respectively) as the dependent variable. Combining 2011 and 2012 biodiversity data produces a much larger sample size which benefits statistical modeling at every level in this research. Table 4.4 shows the RFR error measures (MSR and \%Variance explained) by taxa for models derived using the 2011 and 2012 MODIS indices for both $1 \times 1 \mathrm{~km}$ and $3 \times 3 \mathrm{~km}$ sample landscapes. Model errors for all years (2000-2012) are 
listed in Appendix IV. As can be seen in Table 4.4, \% Var was negative for most of $1 \times 1 \mathrm{~km}$ models, as well as $3 \times 3 \mathrm{~km}$ models of birds and butterflies, which indicates poor ability of the models for predicting gamma diversity. However, $\%$ Var was positive for $1 \times 1 \mathrm{~km}$ plant models for 2012 , as well as for $3 \times 3 \mathrm{~km}$ models of plants and beetles. $\%$ Var increased from $1 \times 1 \mathrm{~km}$ to the $3 \times 3 \mathrm{~km}$ models, indicating that at $3 \times 3 \mathrm{~km}$ a higher proportion of variance was explained by the explanatory variables.

The MSR is an indicator of the difference between the observed and predicted values. It was compared with the range of gamma diversity (maximum - minimum gamma value over all landscapes) for each taxon as shown in Table 4.5. For both landscape extents, MSR was higher than the gamma range for plants while it was lower than the gamma range for beetles, birds and butterflies. MSR values for beetle models were lower for $3 \times 3 \mathrm{~km}$ models compared to $1 \times 1 \mathrm{~km}$ models, while models for birds and butterflies did not show distinct differences with respect to landscape extent.

Following RFR model runs for each taxon in all years, the nine most important variables for each taxon were determined; i.e., only the top nine variables were selected based on the 10:1 observations:variables constraint of the subsequently implemented SWR. Many of the same variables were in the top nine for each taxon, but their order of importance varied from one taxon to another and for $1 \times 1 \mathrm{~km}$ and $3 \times 3 \mathrm{~km}$ landscape extents (Table 4.6). 
Table 4.4. Error measures from random forest regressions of 2011-2012 gamma diversity summarized by taxon for models derived using MODIS phenological indices for 2011 and 2012. The percent variance (\% Var) and mean of squared residuals (MSR) were calculated from random subsets of one third of the reference samples in each tree. RFR runs were implemented for $1 \times 1 \mathrm{~km}$ to $3 \times 3 \mathrm{~km}$ landscapes independently. Range of gamma diversity for each taxon is in brackets beside the taxon name.

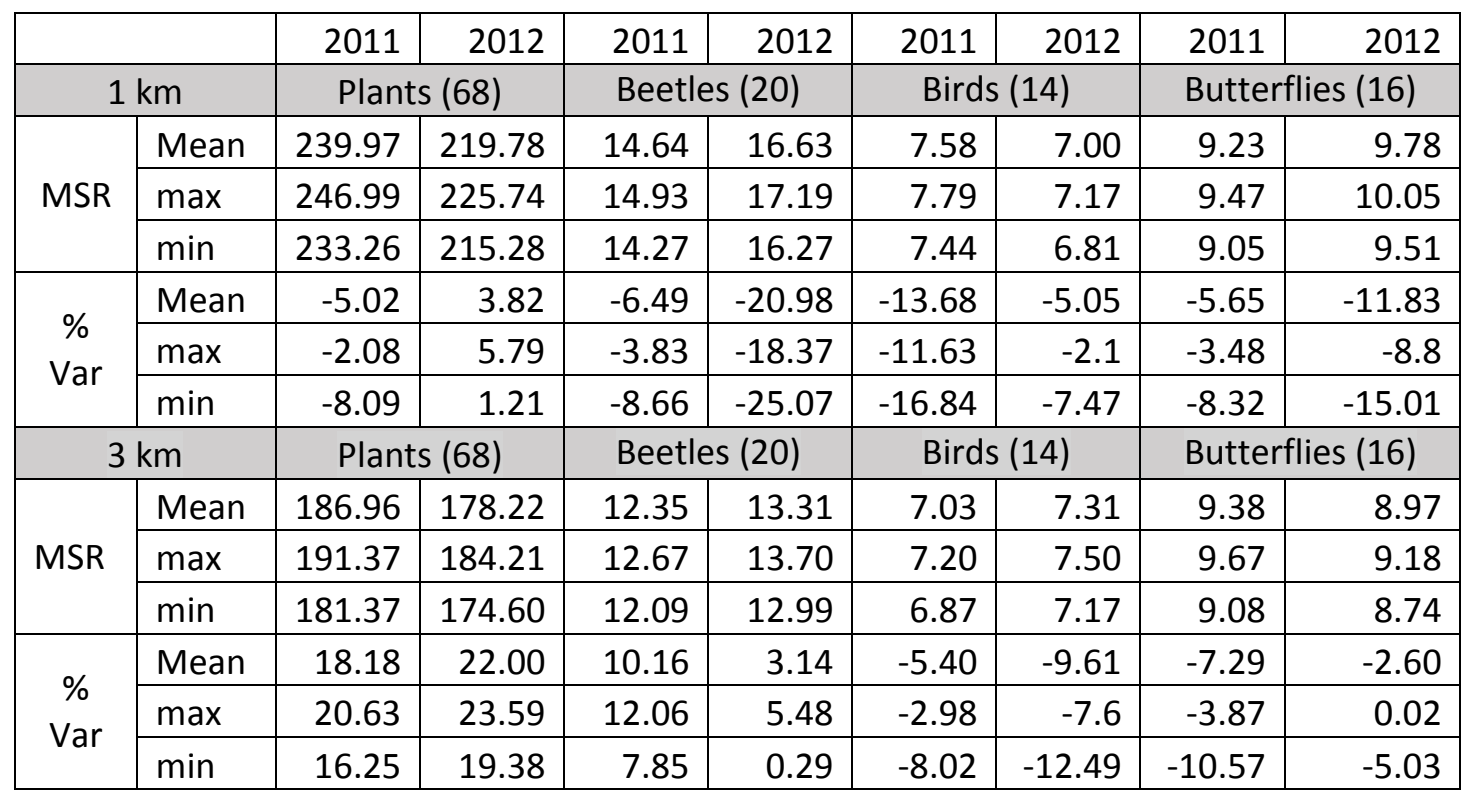

Variables associated with the start of season and end of season (mean and CV of SOS/EOS DOY and of SOS/EOS NDVI), which represent eight of the 22 input variables, occurred most frequently in the top nine variables. For example, in the $1 \times 1 \mathrm{~km}$ models for plants, SOS and EOS variables were amongst the top nine in $20.9 \%$ of models, while in $3 \times 3$ models SOS and EOS variables were amongst the top nine in 50.12\% of models. The percentages of SOS and EOS variables in the top nine for the other taxa in $1 \times 1 \mathrm{~km}$ models were $14.89 \%$ (birds), $23.10 \%$ (beetles) and $28.51 \%$ (butterflies) and in $3 \times 3 \mathrm{~km}$ models were $41.12 \%$ (birds), $56.10 \%$ (beetles) and 26.17\% (butterflies). Despite the generally low strength of many of the RFR models, these results indicated that gamma diversity for these taxa potentially displays some relationship with the average and variability of the time of emergence and senescence, as well as the productivity of vegetation at the time of emergence and senescence. 
In $1 \times 1 \mathrm{~km}$ models, SEAS INT and SEAS AMP were amongst the top nine variables in $25 \%$ of models for beetles, $21 \%$ for birds, $21 \%$ for butterflies and $25 \%$ for plants. This demonstrated the importance of overall net primary productivity of the landscape in species diversity. In addition, the importance of spatial variability of phenological variables was evident in the models since $\mathrm{CV}$ of the variables appeared frequently in the top nine for all taxa in both 1 $\times 1 \mathrm{~km}$ and $3 \times 3 \mathrm{~km}$ models.

\subsubsection{Step-wise multiple regression models}

SWR modelling results demonstrated that phenological and long-term annual changes in vegetation dynamics and productivity are related to farmland biodiversity. The absolute r-values

for $1 \times 1 \mathrm{~km}$ and $3 \times 3 \mathrm{~km}$ models are presented in Figure 4.6. The magnitude of $r$-values increased for all species in most years from $1 \times 1 \mathrm{~km}$ to $3 \times 3 \mathrm{~km}$ extents. The low r-values and lack of statistical significance in the $1 \times 1 \mathrm{~km}$ models could be due to the small number of MODIS pixels (12-16) within the $1 \times 1 \mathrm{~km}$ extent from which the landscape averages of the phenology variables were calculated. For the $3 \times 3 \mathrm{~km}$ extent (with approximately 144 MODIS pixels) a general improvement in model r-values was observed. These results emphasise the effect of landscape extent and sample size (number of pixels) on model performance, especially when using variables derived from coarse spatial resolution data such as MODIS. The outputs of stepwise models for all years are presented in Appendix VI. 
Table 4.5. The nine most important variables selected by random forest regression models for each taxon and the sum of the frequency of their occurrence in models over all 13 years (20002012) and the percentage of occurance over all iterations (1300). Results are presented for $1 \times 1$ $\mathrm{km}$ and $3 \times 3 \mathrm{~km}$ landscapes.

\begin{tabular}{|c|c|c|c|c|c|}
\hline \multicolumn{5}{|c|}{ Beetles } \\
\hline \multicolumn{2}{|c|}{$1 \mathrm{~km}$} & \multicolumn{3}{c|}{$3 \mathrm{~km}$} \\
\hline Variable & Sum & $\%$ & Variable & Sum & $\%$ \\
\hline SEAS INT CV & 524 & 40.3 & SOS DOY CV & 589 & 45.3 \\
\hline $\begin{array}{c}\text { MEAN SEAS } \\
\text { DUR }\end{array}$ & 405 & 31.1 & SOS NDVI CV & 571 & 43.9 \\
\hline $\begin{array}{c}\text { SOS NDVI CV } \\
\text { MEAN SEAS INT }\end{array}$ & 360 & 27.7 & MEAN NDVI & 539 & 41.4 \\
\hline \begin{tabular}{c} 
SEAS DUR CV \\
\hline $\begin{array}{c}\text { MEAN SOS } \\
\text { NDVI }\end{array}$
\end{tabular} & 299 & 23.0 & MEAN SOS NDVI & 430 & 33.0 \\
\hline $\begin{array}{c}\text { MEAN EOS } \\
\text { NDVI }\end{array}$ & 250 & 19.2 & MAX DOY CV & 236 & 18.1 \\
\hline $\begin{array}{c}\text { MEAN SEAS } \\
\text { AMP }\end{array}$ & 196 & 15.0 & MEAN MAX NDVI & 231 & 17.7 \\
\hline $\begin{array}{c}\text { MEAN MAX } \\
\text { NDVI }\end{array}$ & 159 & 12.2 & MEAN SEAS DUR & 201 & 15.4 \\
\hline
\end{tabular}

\begin{tabular}{|c|c|c|c|c|c|}
\hline \multicolumn{6}{|c|}{ Birds } \\
\hline \multicolumn{3}{|c|}{$1 \mathrm{~km}$} & \multicolumn{3}{|c|}{$3 \mathrm{~km}$} \\
\hline Variable & Sum & $\%$ & Variable & Sum & $\%$ \\
\hline SEAS AMP CV & 437 & 33.6 & SOS NDVI CV & 529 & 40.6 \\
\hline SEAS GRE CV & 367 & 28.2 & EOS NDVI CV & 434 & 33.3 \\
\hline SOS DOY CV & 357 & 27.4 & MEAN NDVI & 398 & 30.6 \\
\hline SEAS INT CV & 300 & 23.0 & $\begin{array}{c}\text { MEAN SEAS } \\
\text { GRE }\end{array}$ & 387 & 29.7 \\
\hline MEAN SEAS DUR & 294 & 22.6 & $\begin{array}{c}\text { MEAN EOS } \\
\text { NDVI }\end{array}$ & 343 & 26.3 \\
\hline SEAS DUR CV & 265 & 20.3 & MAX DOY CV & 309 & 23.7 \\
\hline EOS NDVI CV & 224 & 17.2 & EOS DOY CV & 298 & 22.9 \\
\hline MEAN MAX DOY & 183 & 14 & NDVICV & 234 & 18.0 \\
\hline MEAN SEAS INT & 155 & 11.9 & $\begin{array}{c}\text { MEAN SEAS } \\
\text { DUR }\end{array}$ & 226 & 17.3 \\
\hline
\end{tabular}

\begin{tabular}{|c|c|c|c|c|c|}
\hline \multicolumn{6}{|c|}{ Butterflies } \\
\hline \multicolumn{3}{|c|}{$1 \mathrm{~km}$} & \multicolumn{3}{|c|}{$3 \mathrm{~km}$} \\
\hline Variable & Sum & $\%$ & Variable & Sum & $\%$ \\
\hline $\begin{array}{l}\text { MEAN SEAS } \\
\text { INT }\end{array}$ & 538 & 41.3 & MEAN SEAS DUR & 521 & 40.0 \\
\hline SEAS INT CV & 389 & 29.9 & MEAN MAX NDVI & 490 & 37.7 \\
\hline MEAN NDVI & 387 & 29.7 & MEAN NDVI & 467 & 35.9 \\
\hline $\begin{array}{c}\text { MEAN EOS } \\
\text { NDVI }\end{array}$ & 358 & 27.5 & MEAN EOS NDVI & 439 & 33.7 \\
\hline EOS NDVI CV & 324 & 24.9 & SOS DOY CV & 360 & 27.7 \\
\hline SOS NDVI CV & 299 & 23.0 & NDVI CV & 290 & 22.3 \\
\hline $\begin{array}{c}\text { MEAN SEAS } \\
\text { DUR }\end{array}$ & 166 & 12.7 & EOS NDVI CV & 222 & 17.0 \\
\hline MAX DOY CV & 133 & 10.2 & SEAS DUR CV & 196 & 15.0 \\
\hline $\begin{array}{c}\text { MEAN SOS } \\
\text { NDVI }\end{array}$ & 131 & 10.0 & MAX DOY CV & 176 & 13.5 \\
\hline
\end{tabular}

\begin{tabular}{|c|c|c|c|c|c|}
\hline \multicolumn{6}{|c|}{ Plants } \\
\hline \multicolumn{3}{|c|}{$1 \mathrm{~km}$} & \multicolumn{3}{|c|}{$3 \mathrm{~km}$} \\
\hline Variable & Sum & $\%$ & Variable & Sum & $\%$ \\
\hline MEAN SEAS INT & 747 & 57.4 & $\begin{array}{l}\text { MEAN SOS } \\
\text { DOY }\end{array}$ & 945 & 72.7 \\
\hline MAX NDVI CV & 494 & 38 & $\begin{array}{c}\text { MEAN SEAS } \\
\text { INT }\end{array}$ & 793 & 61.0 \\
\hline MEAN SEAS DUR & 374 & 28.7 & $\begin{array}{c}\text { MEAN SOS } \\
\text { NDVI }\end{array}$ & 673 & 51.7 \\
\hline SOS NDVI CV & 289 & 22.2 & $\begin{array}{c}\text { MEAN SEAS } \\
\text { DUR }\end{array}$ & 411 & 31.6 \\
\hline SEAS GRE CV & 243 & 18.7 & SOS NDVI CV & 240 & 18.4 \\
\hline SOS DOY CV & 199 & 15.3 & $\begin{array}{c}\text { MEAN SEAS } \\
\text { AMP }\end{array}$ & 225 & 17.3 \\
\hline MAX DOY CV & 184 & 14.1 & NDVI CV & 199 & 15.3 \\
\hline MEAN EOS DOY & 175 & 13.4 & SOS DOY CV & 97 & 32.3 \\
\hline MEAN SOS NDVI & 154 & 11.8 & SEAS INT CV & 83 & 6.3 \\
\hline
\end{tabular}



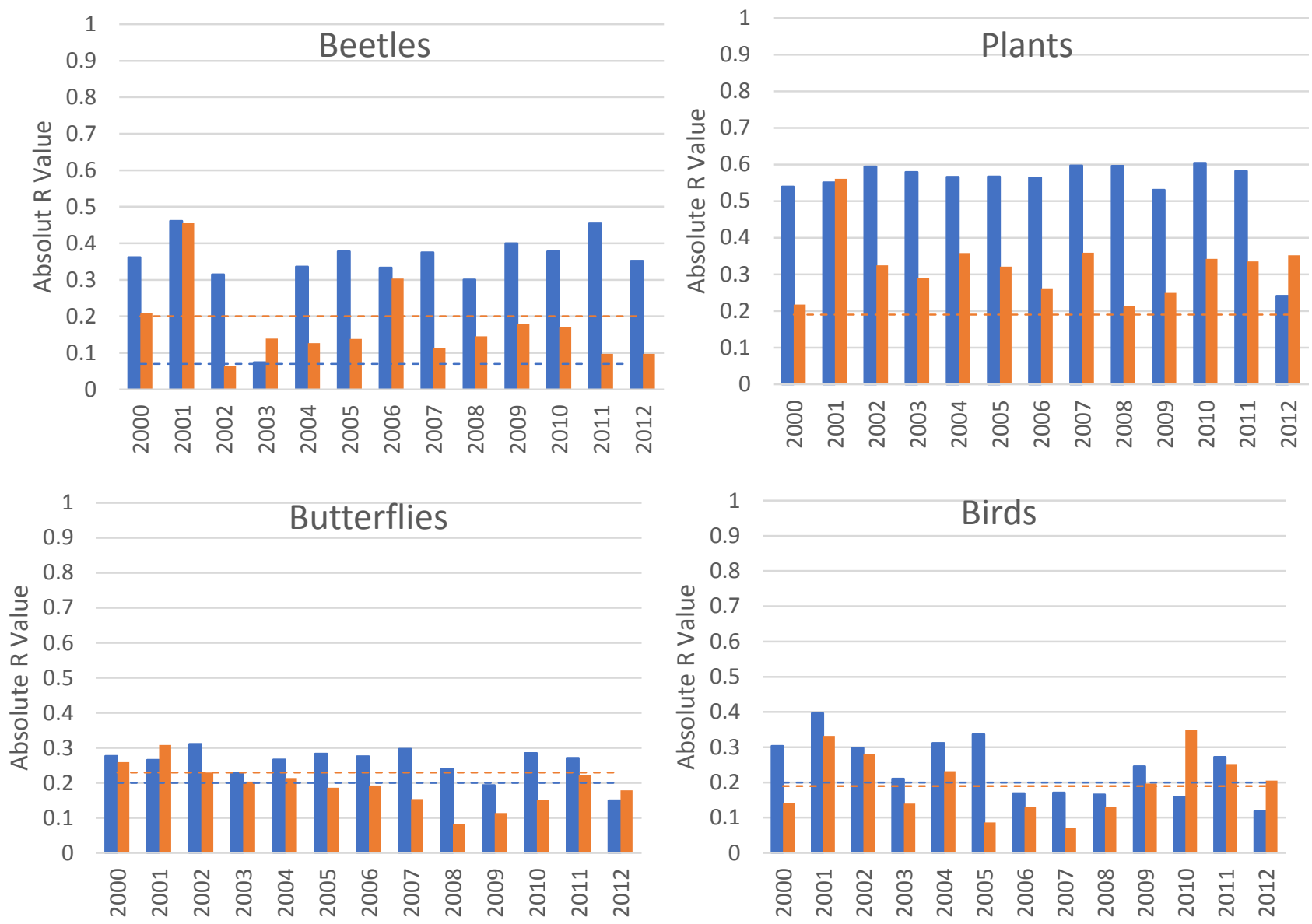

Figure 4. 6. Absolute r-value for all models. Orange represents $1 \times 1 \mathrm{~km}$ models and blue represents $3 \times 3 \mathrm{~km}$ models. Significant variables in step-wise models and the strength and direction of their relationships with gamma diversity. Dotted lines represent $r$ when $p=0.05$ for each corresponding landscape extent.

The strength and direction of the relation between the explanatory variables selected in SWR and gamma diversity were indicated by the r-value of each variable. The variables selected, the model r-value and the direction of the variable effects ( + or -$)$ varied among years for each taxon but there was some consistency with respect to direction. Since $1 \times 1 \mathrm{~km}$ models were not of statistical significance in most years and taxa, the r-values are presented hereafter only for the $3 \times 3 \mathrm{~km}$ models. The number of times that each variable was entered into models for each taxon is presented in Table 4.7. 
Table 4.6. MODIS phenological variables entered into SWR models and the number of years (out of 13) each variable was entered into a model for each taxon and $3 \times 3 \mathrm{~km}$ landscapes

\begin{tabular}{|l|c|c|c|c|}
\hline & Birds & Beetles & Butterflies & Plants \\
\hline Mean NDVI & & 6 & 1 & \\
\hline NDVI CV & 4 & & & 4 \\
\hline Mean SOS NDVI & & 4 & & 3 \\
\hline SOS NDVI CV & & 2 & & 3 \\
\hline Mean SOS DOY & & & & 5 \\
\hline SOS DOY CV & & 1 & 3 & 1 \\
\hline Mean EOS NDVI & & 3 & 6 & \\
\hline EOS NDVI CV & & & 1 & \\
\hline Mean EOS DOY & & & & \\
\hline EOS DOY CV & 1 & & & \\
\hline Mean MAX NDVI & & 1 & & \\
\hline MAX NDVI CV & & & & \\
\hline Mean MAX DOY & & & & \\
\hline MAX DOY CV & 4 & & & \\
\hline Mean SEAS DUR & & 1 & 1 & 3 \\
\hline SEAS DUR CV & 4 & & 1 & \\
\hline Mean SEAS INT & & & & 2 \\
\hline SEAS INT CV & & & & \\
\hline Mean SEAS AMP & & & & 1 \\
\hline SEAS AMP CV & & & & \\
\hline Mean SEAS GRE & 4 & & & \\
\hline SEAS GRE CV & & & & \\
\hline
\end{tabular}

The significance, $\mathrm{r}$-values and direction of each variable in $3 \times 3 \mathrm{~km}$ models are presented below for each taxon independently. The graphs (Figure 4.6 to 4.9) present r-values with 95\% confidence intervals.

\subsection{Plants}

Significant models were created for plants in all years. Overall, 8 variables entered the models in different years: NDVI CV, SOS NDVI CV, Mean SOS DOY and SOS DOY CV display a significant negative relationship with plant gamma diversity, while Mean SEAS AMP, Mean SEAS INT, Mean SEAS DUR and Mean SOS NDVI demonstrate a significant positive relationship. The selected variables entered models in the following years: NDVI CV - 2004, 2005, 2006, 2012; Mean SOS NDVI - 2000, 2001, 2009; Mean SOS DOY - 2000, 2003, 2007, 2010, 2011; SOS DOY CV- 2007; SOS NDVI CV-2001, 2003, 2008; Mean SEAS DUR- 2004, 
2005, 2006; Mean SES Amp - 2002; Mean SEAS INT 2002, 2008. The r-values for these variables in the stepwise model for each year are presented in Figure 4.7. Only five models were single variable models $(2000,2009,2010,2011$ and 2012), while seven were two variables models (2001, 2003, 2004, 2005, 2006, 2007 and 2008) and one (2002) was a three variable model.

Plant models demonstrated the strongest relationships between gamma diversity and the input explanatory variables. The strong negative relationship with Mean SOS DOY (the most frequently entered variable in the models) and SOS DOY CV suggests that later vegetation emergence and higher variability in the time of vegetation emergence across the landscape is negatively related to the diversity of non-crop plants. The strong negative relationship of NDVI CV and SOS NDVI CV also demonstrates that higher variability of NDVI over the landscape at the time of vegetation emergence, as well as over the whole growing season, is negatively related to the diversity of wild plants. These relationships are associative and probably not causal. High spatial variability in NDVI typically means that some areas of a given landscape have full or nearly full vegetation cover and others have distinctly less than full cover. In landscapes exhibiting such a spatial variability in productivity, fewer plant species become established in a given growing season. The other variables demonstrated strong positive relationships with plant gamma. In other words, a longer growing season, higher seasonal vegetation productivity and higher vegetation productivity at the time of onset of greenness were associated with higher plant gamma diversity. 


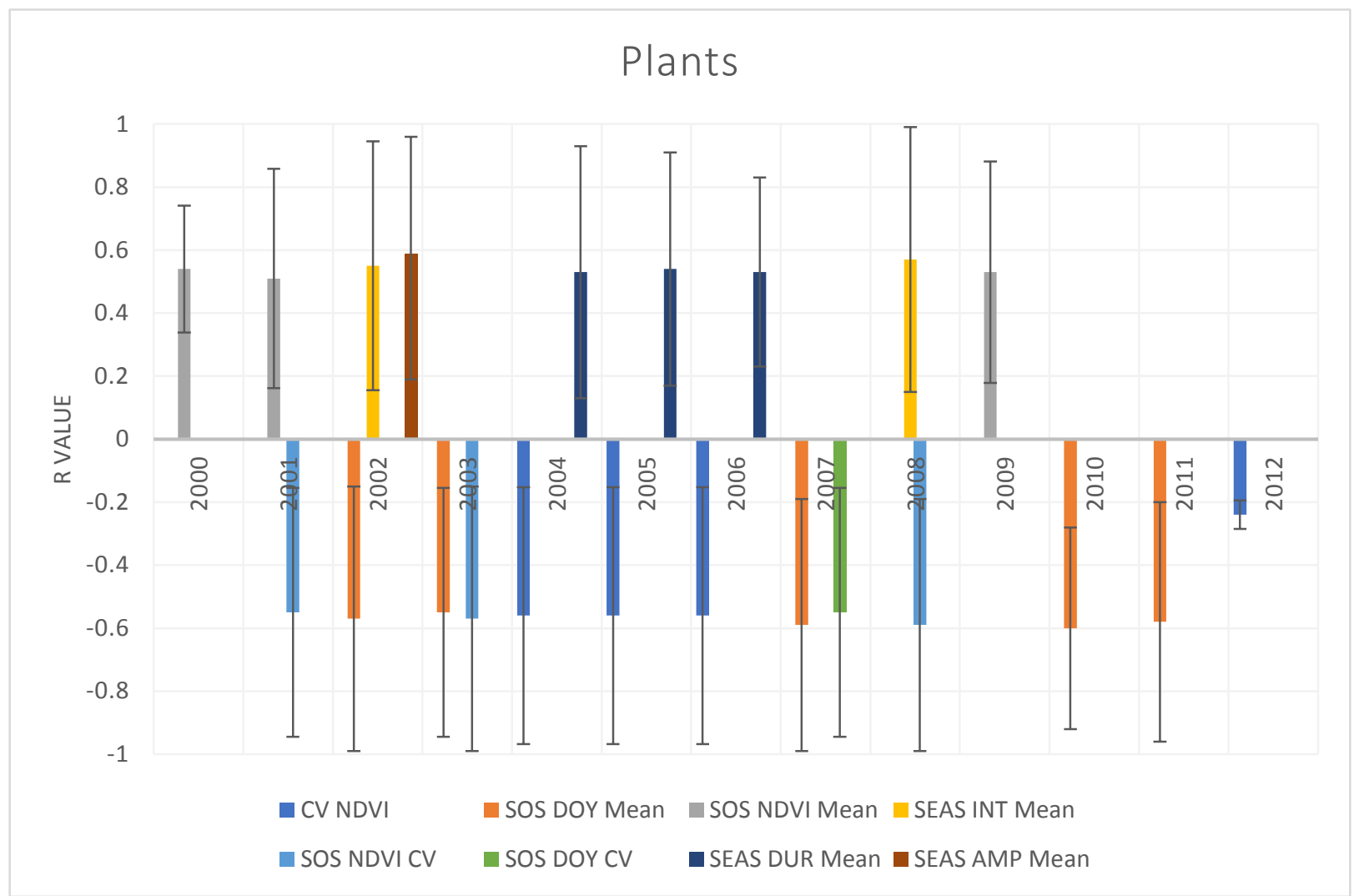

Figure 4.7. R-values and variables entered into SWR models of gamma diversity in each corresponding year for plants and 3 x $3 \mathrm{~km}$ landscapes. Error bars present 95\% confidence intervals.

\subsection{Beetles}

Among the selected predictors that entered beetle models, Mean NDVI was the most frequent, occurring in 6 of 13 (46.15\%) models (2001, 2003, 2004, 2007, 2011 and 2012) and demonstrating a positive relationship in all years. It was the only variable entered in 2003, 2004, 2007 and 2012 models. Other variables which entered the models were: SOS NDVI CV in 2005 and 2011, Mean SOS NDVI in 2002, 2006, 2009 and 2010 and Mean SEAS DUR in 2008, which all demonstrated a significant positive relationship with beetle gamma diversity. SOS DOY CV and Mean Max NDVI demonstrated a significant negative relationship in 2001 and 2011, respectively, and Mean EOS NDVI displayed a positive relationship in 2000 and 2005 and a negative relationship in 2009. These results are presented in Figure 4.8. 
Nine of 13 models were single variable models (2000, 2002, 2003, 2004, 2006, 2007, 2008, 2010 and 2012), while three were two variables models (2001, 2005 and 2009) and 2001 was the only model with three variables entered.

The significant positive relationship of one or more of Mean NDVI, Mean SOS NDVI and SOS NDVI CV with beetle gamma diversity in models from all years except 2008 demonstrated that more productive and variable landscapes at the time of vegetation greenness onset and over the whole growing season supported higher beetle diversity. All other variables did not enter models frequently enough to warrant further discussion and interpretation.

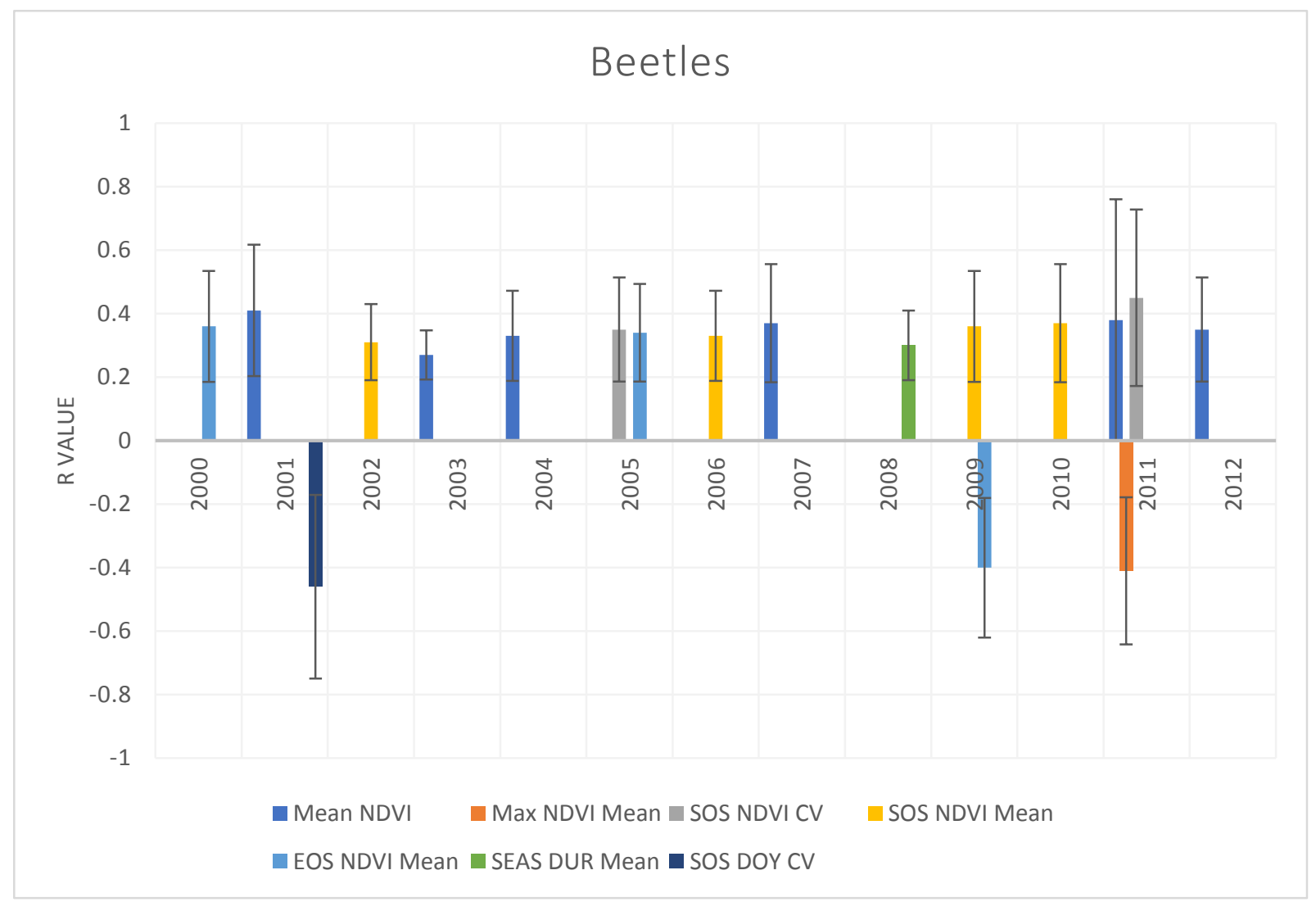

Figure 4.8. R-values and variables entered into SWR models of gamma diversity in each corresponding year for beetles and 3 x $3 \mathrm{~km}$ landscapes. Error bars present $95 \%$ confidence intervals. 


\subsection{Birds}

Eleven significant models were produced for bird gamma diversity, seven of which were single variable $(2002,2003,2008,2009,2010,2011$ and 2012), and four of which were two variable models $(2000,2001,2004$ and 2005). The strength of the relationships was generally weaker compared to plants and beetles. All variables which entered the models demonstrated a negative relationship with bird gamma diversity. These variables were: Mean SEAS DUR (2003, 2010, 2011), EOS DOY CV (2002), Max DOY CV (2001, 2004, 2005), NDVI CV (2000, 2008, 2009, 2012) and Mean SEAS GRE $(2000,2001,2004,2005)$. These results are presented in Figure 4.9.

Negative relationships of bird gamma diversity with CV of MAX DOY, EOS DOY and SEAS DUR indicated that the variability in the day of important phenological events, as well as the variability in the length of growing season across sample landscapes can negatively affect birds. Negative relationships of bird gamma with NDVI CV as a representation of variability in vegetation productivity across landscapes demonstrated the importance of stability of landscape productivity for bird species. This result was in agreement with that of Duro et al. (2014), which found a significant negative relation between bird diversity and NDVI CV using mid-summer Landsat 5 imagery. As stated above for plants, landscapes with higher NDVI CV probably vary distinctly in the amount of vegetation across the landscape. In such landscapes without more spatially uniform vegetation cover, some bird species may not find the protection or food sources they need, resulting in lower bird gamma diversity.

The above results demonstrated that variability in the phenological events and overall productivity of landscapes due to presence of different crop types (compositional heterogeneity) did not benefit bird diversity. This contradicted the habitat heterogeneity hypothesis by showing that more heterogeneous landscapes may fail to meet the habitat requirements of birds at the right timing (Benton et al., 2003). In addition, birds demonstrated a negative relationship with Mean 
SEAS GRE which indicated that a high overall greenness of landscapes can negatively affect birds. This could be due to habitat preference of birds (uplands, wetlands, etc.) which may favour less green landscapes to meet their biological needs.

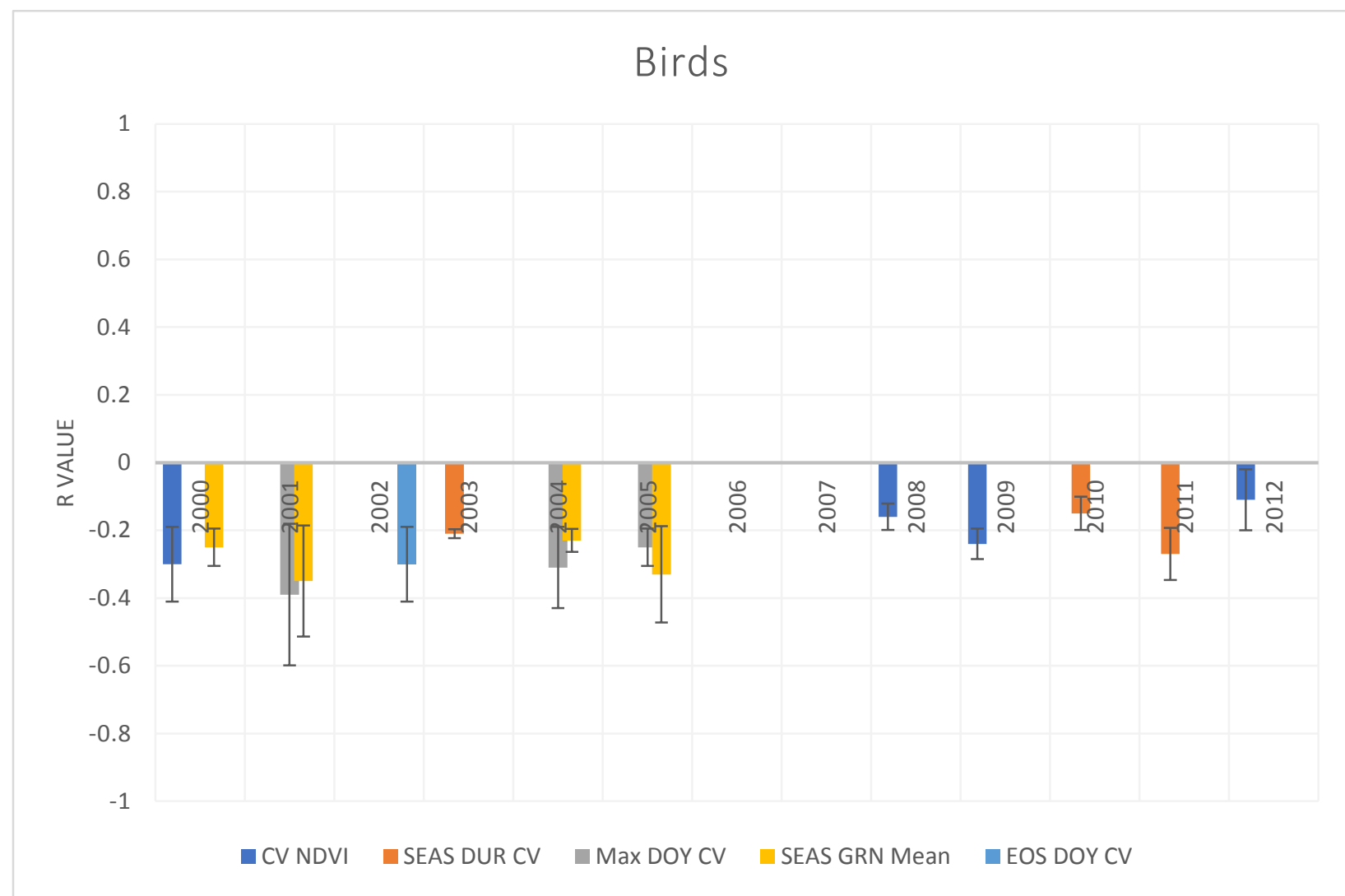

Figure 4. 9. R-values and variables entered into SWR models of gamma diversity in each corresponding year for birds and 3 x $3 \mathrm{~km}$ landscapes. Error bars present $95 \%$ confidence intervals.

\subsection{Butterflies}

Similar to birds, butterfly models were weaker, all of which were single variable. They demonstrated positive relationships with Mean EOS NDVI (most frequently entered variable) in 2000, 2005, 2006, 2009, 2010, and 2011 and negative relationships with SOS DOY CV in 2004, 
2007 and 2008. They also demonstrated a positive relationship in a single year with Mean NDVI (2012), Mean SEAS DUR (2001), SOS NDVI CV (2002) and SEAS DUR CV (2003). These results are presented in Figure 4.10.

Positive relationships of Mean EOS NDVI and Mean NDVI with butterfly gamma diversity demonstrated the positive effect of higher NDVI at the time of vegetation senescence and during the growing season on butterflies. Other variables had occurred in less than one third of the models and the results may not be generalized for all years.

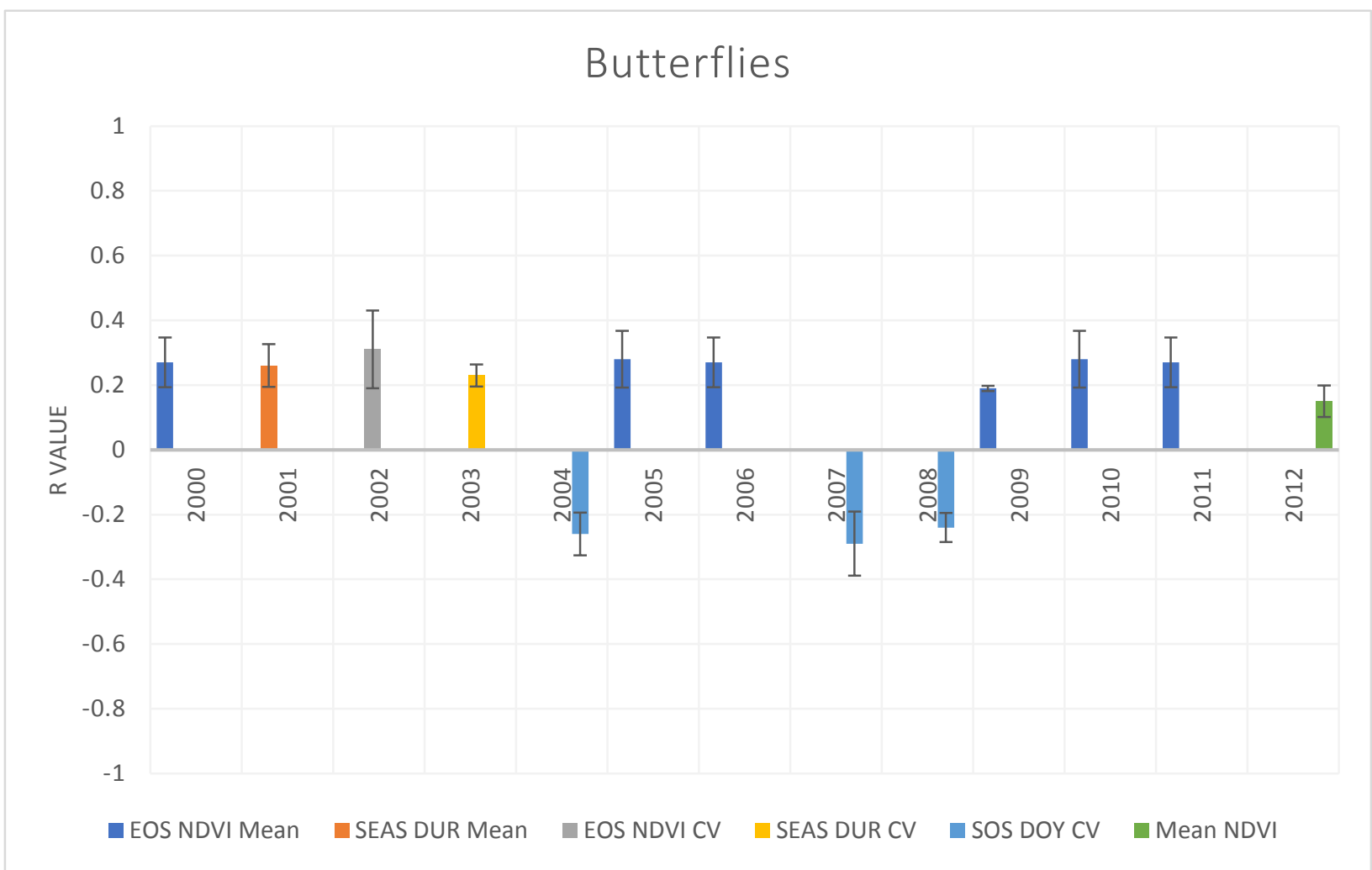

Figure 4. 10. R-values and variables entered into SWR models of gamma diversity in each corresponding year for butterflies and $3 \times 3 \mathrm{~km}$ landscapes. Error bars present $95 \%$ confidence intervals.

\subsubsection{Model residuals}

Model residuals were normally distributed for all models. Residual plots demonstrated a scattered distribution along the $\mathrm{x}$-axis (the predictor), suggesting a linear and homoscedastic relationship between the predictors and gamma diversity. The Breusch-Pagan heteroscedasticity test (Breusch 
and Pagan, 1979) was run on the model residuals using a significance level of $p=0.05$. The $p$ value range from 0.47 to 0.08 suggested non-significant and homoscedastic relationships. As an example, the residual plot of Mean EOS NDVI against beetle gamma diversity in 2012 is presented in Figure 4.11. The residual plots of other models followed a similar scattered pattern, also indicative of a homoscedastic relationship.

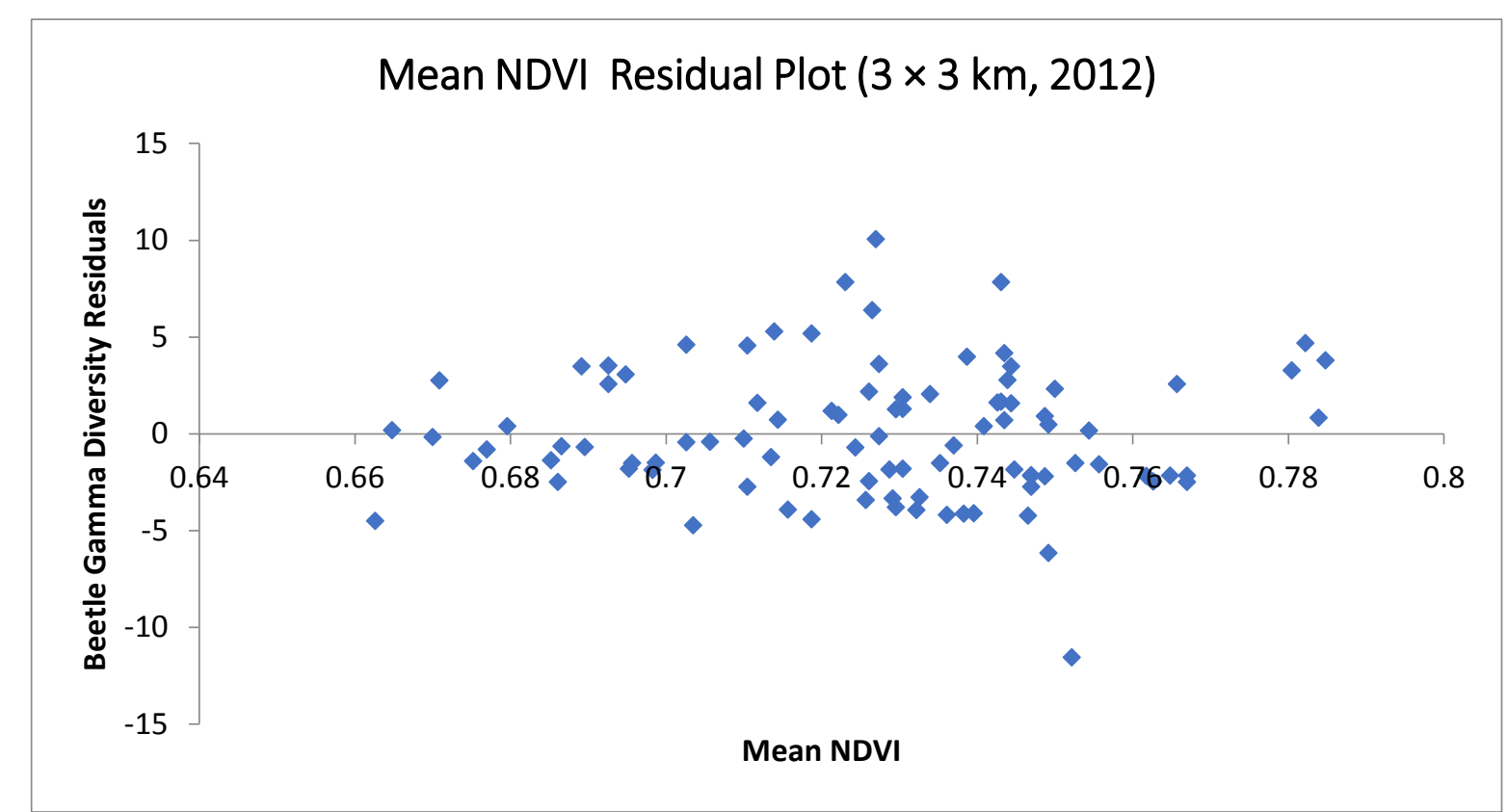

Figure 4. 11. Example residual plot of beetle gamma diversity model for Mean EOS NDVI in 2012

\subsubsection{Alternative statistical methods}

Comparing the results of AICc and LMG with those of RFR demonstrated strong agreement for most $3 \times 3$ beetle and plant models in 2000, 2006 and 2011 . Overall, $77.6 \%$ and $89.3 \%$ of the top nine most important variables in LMG and AICc were in agreement with those of RFR in the selected subset of data. The order of the relative importance of variables in LMG and AICc were also compared to that of RFR and the variable(s) entered SWR and a strong agreement for most models was found. For example, for the $3 \times 3 \mathrm{~km}$ plant model in 2000, Mean SOS NDVI appeared 
as the most significant variable with lowest AICc and showed the sixth highest $\mathrm{R}^{2}$ in $\mathrm{LMG}$, while it was selected by RFR in 47 of the 100 iterations and it was the only variable which entered SWR model. In the 2006 model, Mean SEAS DUR was the most significant variable with lowest AICc and showed the highest $\mathrm{R}^{2}$ in $\mathrm{LMG}$, while it was selected in 85 RFR iterations and it was one of the variables which entered SWR model. In the 2011 model, Mean SOS DOY appeared as the most significant variable with lowest AICc and demonstrated the fourth highest $\mathrm{R}^{2}$ in $\mathrm{LMG}$, while it was selected as the most significant variable in 100 RFR iterations and it was the only variable which entered the model in SWR.

For the $3 \times 3 \mathrm{~km}$ beetle model in 2000, Mean EOS NDVI was the most significant variable with lowest AICc and showed the sixth highest $\mathrm{R}^{2}$ in $\mathrm{LMG}$, while it was selected in 94 RFR iterations and it appeared as the only variable which entered SWR. In 2006, Mean SOS NDVI was the most significant variable with lowest $\mathrm{AICc}$ and it demonstrated the third highest $\mathrm{R}^{2}$ in LMG, while it appeared in 57 RFR iterations and it was the only variable which entered the SWR model in that year. Finally, in the 2011 model, Mean SEAS INT appeared as the most significant variable in AICc and showed the seventh highest $\mathrm{R}^{2}$ in $\mathrm{LMG}$, while it appeared as the most significant variable in 32 RFR iterations and did not enter the SWR model.

High compatibility of the results of these alternative statistical methods with those of RFR and SWR raised confidence in the RFR and SWR results of this study.

\subsubsection{Evaluating potential lag effects}

The analysis of potential lag effects was conducted on $3 \times 3 \mathrm{~km}$ sample landscapes. For plants and birds, NDVI CV, for beetles Mean NDVI and for butterflies Mean EOS NDVI were selected as the best variables which entered individual models most frequently in SWR. The lag effect was tested for each species individually by creating models as explained in 4.5.3.1.5. Beetles, birds 
and butterflies did not show any patterns of lag effect, as adding the variable of past years to the model caused model inflation and decreases in the Pearson r. For plants, on the other hand, the rvalue was low for M1, M2, M3 M4 and M5 but demonstrated a jump in M6, where the NDVI CV of 2007 was added (Figure 4.12). This suggested a stronger relation between 2007 NDVI CV and gamma diversity of plants in 2011-2012. Different scenarios were tested to verify this. For example, plant gamma diversity was modeled against NDVI CV of 2012 and 2007 and an r-value 0.4 higher than M1 $(r=0.21)$ and equal to M6 was obtained.

The potential effect of climate variation on this time lagged response was investigated. No extreme phenological events were evident in 2007 (Figure 6.1) but total July precipitation (86 $\mathrm{mm}$ ) was $50 \%$ higher in 2007 than the $2000-2012$ average and $20-50 \%$ lower than the $2000-2012$ average for the years following 2007 (except 2009, Table 6.1).

In addition, the potential effect of landscape change was tested by removing NDVI CV of 2007 from the models. It was assumed that if major landscape changes (such as removal of natural and semi-natural patches and hedgerows and an overall switch in crop types from hay to corn and soybean) had occurred after 2007, all the years prior to 2007 would reflect the same landscape condition as 2007. No significant change in model performance was noted, which confirmed the above assumption about potential landscape change after 2007. Statistics Canada, Census of Agriculture (2016) reported a major shift in crop composition in Ontario in the late 2000s from less heavily managed crops such as hay, alfalfa and perennial plants with higher seasonal variability to more intensively managed crops such as corn and soybean with less seasonal variability. Intensive farming practices such as tillage and use of herbicides are associated with fewer non-crop plants in the landscapes. The lag effect models that were tested in this study suggest a delayed response of non-crop plants to this change in farming practices and crop types. 


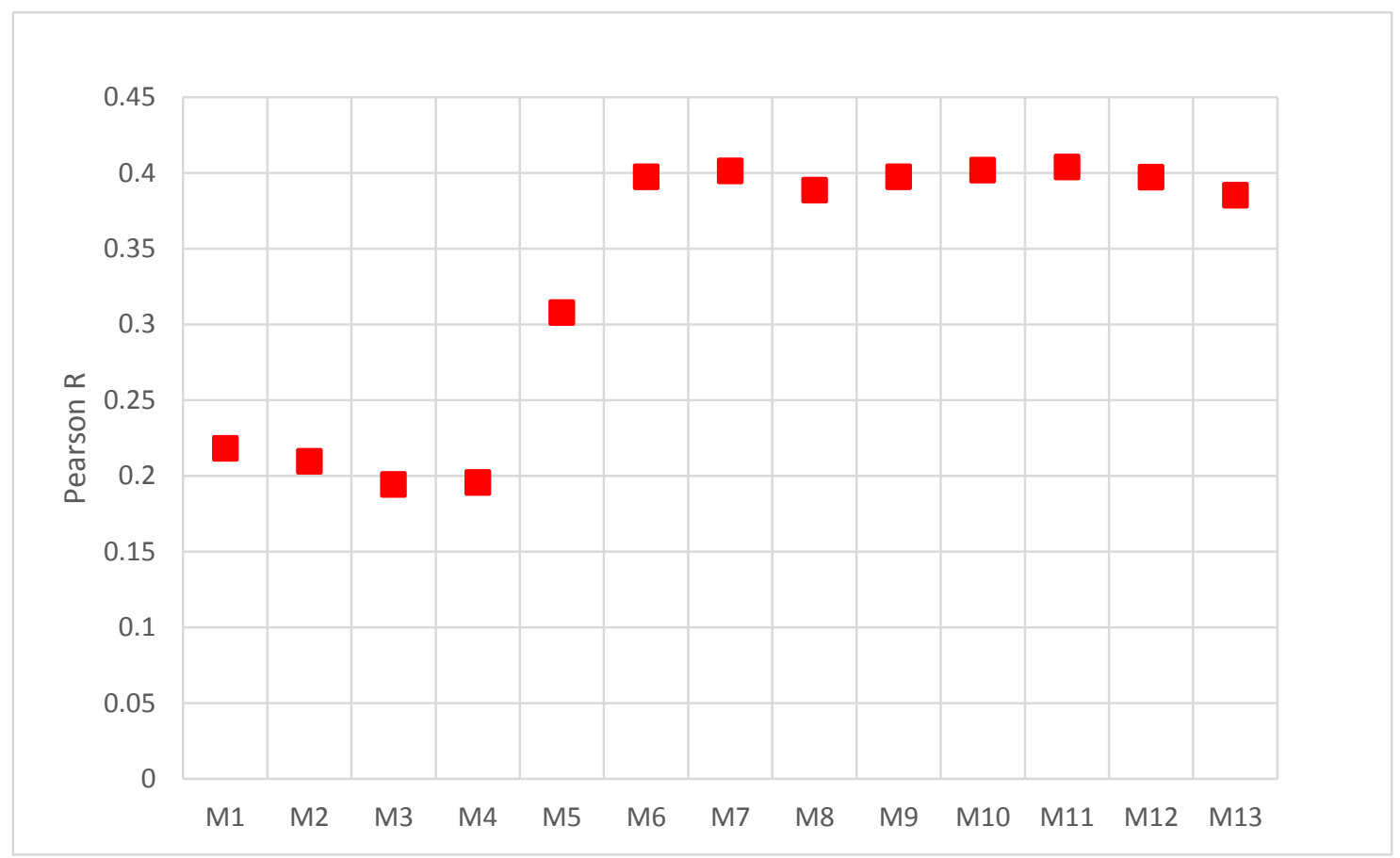

Figure 4. 12. Pearson $r$ for lag effect models of NDVI CV against plant gamma diversity. A jump in the $r$ value was evidenced in M6 where NDVI CV of 2007 was added to the model.

\subsection{Discussion}

\subsubsection{Interpretation of main findings and relations to previous work}

The first objective of this study was to evaluate relationships between vegetation phenology derived from MODIS NDVI weekly data from 2000 to 2012 and biodiversity of mixed agricultural landscapes of Eastern Ontario surveyed in 2011 and 2012. The results of RFR and stepwise models suggested that changes in vegetation phenology and productivity are related to the diversity of farmland species. These results are in agreement with those of Vina et al. (2016) who found that land surface phenology (flowering, fruiting, senescence) derived from MODIS VARI data could be used as an indicator of plant diversity and Hurlbert and Haskell (2003) which demonstrated that bird diversity is related to seasonal changes in NDVI. Other examples, however, were not found; the relationships between vegetation phenology and biodiversity have 
not been widely studied and further investigation in other farmland environments is required to confirm the relationships found here.

The second objective of this study was to determine the most significant predictor of biodiversity in RFR and stepwise models. Overall, 10400 RFR models were produced for four selected taxa (1300 models for each taxon at each landscape extent). Variables related to the spatial average and variability in time of onset of greenness and vegetation senescence within landscapes (Mean and CV of SOS DOY and EOS DOY, respectively), and the spatial average and variability of vegetation productivity at these two times (Mean and CV of SOS NDVI and EOS NDVI, respectively) occurred as significant variables in $32.6 \%$ of the models. In addition, 104 stepwise models were created (13 for each taxon at each landscape extent), 70 (67.3\%) of which were of statistical significance (18 beetles, 17 birds, 13 butterflies, 22 plants). Mean EOS NDVI and Mean SOS NDVI were the most frequent variables entered and occurred in $22.85 \%$ of all significant models, while NDVI CV and Mean NDVI entered 21.4\% of all significant models.

The results of stepwise models revealed that Mean EOS NDVI and Mean NDVI were positively related to the gamma diversity of butterflies and beetles (except beetles in 2009), indicating that higher overall vegetation productivity and higher vegetation productivity at the end of the growing season were associated with higher beetle and butterfly species diversity. Mean SOS NDVI was positively related to beetle and plant gamma diversity, revealing that higher vegetation productivity at the start of season is associated with higher diversity of these taxa. NDVI CV demonstrated significant negative relationships with gamma diversity of plants and birds, indicating that increased spatial variability of vegetation productivity across a landscape may not be beneficial for wild plant and bird diversity. 
The third objective of this study was to identify the taxon or taxa which demonstrate the strongest relationship with the MODIS-derived phenological variables. The results of stepwise models revealed that wild plants created the strongest models as all $1 \times 1 \mathrm{~km}$ and $3 \times 3 \mathrm{~km}$ models were of statistical significance and the $r$ values of the predictors which entered the models were greater than those of other taxa. Plants demonstrated strong negative relationships with the average day of onset of greenness across the landscape. In other words, if leaf emergence is later in the season, perhaps due to climate or weather driven factors, the diversity of non-cropped plants is lower. Beetles produced the second strongest models with $92.3 \%$ of $3 \times 3 \mathrm{~km}$ models and $23 \%$ of $1 \times 1 \mathrm{~km}$ models being significant. They demonstrated a positive relationship with the average and variability of NDVI across the landscape, meaning that landscapes that have more vegetation productivity over the growing season and more spatial variability of that productivity can support more beetle species. Birds and butterflies models were generally weaker with lower r values, but $53.8 \%$ and $61.5 \%$ of $1 \times 1 \mathrm{~km}$ and $3 \times 3 \mathrm{~km}$ models, respectively, were still significant for birds, and $23 \%$ and $84.6 \%$ of $1 \times 1 \mathrm{~km}$ and $3 \times 3 \mathrm{~km}$ models, respectively, were significant for butterflies.

These results are in agreement with previous studies that used the same field biodiversity data but focussed on spatial landscape metrics instead of temporal phenological metrics as in this research. Duro et al. (2014) used metrics of NDVI derived from Landsat 5 such as mean, mean range in summer and CV. Fahrig et al. (2015) used discrete metrics derived from a land cover map such as log of mean field size, Shannon diversity index of crops and percentage of cropped area. Fairbrother (2016) used 30 landscape metrics extracted using the Fragstat system (McGarigal and Marks, 1995) such as Shannon diversity index, mean field size, total edge, interspersion and juxtaposition indices, etc.). They also found that most of the bird and butterfly models were statistically insignificant, while the plant models in Duro et al. (2014) and 
Fairbrother et al. (2016) and plant and beetle models in Fahrig et al. (2015) demonstrated significant relationships.

Furthermore, the importance of wild plants, birds, pollinators (butterflies and bees) and invertebrates, including beetles in agro-ecosystems has been emphasized and relationships between the diversity of these taxa and landscape metrics has been widely studied. Examples of such studies were presented in detail in Chapter 2. (Freemark et al., 2001; Benton et al., (2003); Albrecht et al., 2007; Harwood et al., 2009; Filippi-Codaccioni et al., 2010; Steckel et al., 2014). Significant relationships of similar strength were found for wild plant and beetle diversity against landscape metrics as for those of this study (Freemark et al., 2001; Albrecht et al., 2007; Carvalheiro et al., 2011; Harwood et al., 2009), while other studies on birds and pollinators (Benton et al., 2003; Steckel et al., 2014) found stronger and more significant relationships between species diversity and landscape metrics compared to those of this study.

The fourth objective of this study was to test the relative effect of landscape extent on the relationship between vegetation phenology and farmland biodiversity by modeling vegetation phenology metrics extracted from $1 \times 1 \mathrm{~km}$ and $3 \times 3 \mathrm{~km}$ landscapes against biodiversity. Most of the $1 \times 1 \mathrm{~km}$ models were not statistically significant, whereas most of the $3 \times 3 \mathrm{~km}$ models were $(\mathrm{p} \leq 0.05)$. This demonstrates the impact of sample landscape size on model performance, especially when coarse spatial resolution data such as MODIS are used. This result is in agreement with that of Costanza et al. (2011). They studied the relationship between local plant richness and environmental heterogeneity (abiotic environmental variables, variables related to vegetation productivity and land cover classes) at multiple spatial scales for selected plots and their surroundings and found that measures of heterogeneity were more strongly correlated with local plant richness at larger extents. In addition, Hurlburt and Haskell (2003) found that the strength of the relationship between avian richness and environmental variables was influenced by the 
spatial resolution of the data and different environmental variables create better biodiversity models at coarser spatial resolution, while others perform better at finer spatial resolution. Katayama et al. (2014) also found that the relationship between avian richness and landscape heterogeneity (characterized as Simpson diversity index of land cover types, edge density and the proportion of forest cover) was a function of sample landscape extent. They found that total avian species richness demonstrated a stronger positive relationship with landscape heterogeneity at a larger extent, while narrow-range species prefer homogeneous landscape at a smaller scale.

The fifth objective of this study was to determine if there are potential lag effects by comparing the relationships between phenological variables derived for year 2012 with gamma diversity of 2011 and 2012 and adding variables of previous years to the models one by one in a stepwise process. No distinct systematic difference or trend was found in the response of 20112012 biodiversity to past and present landscape metrics was identified for beetles, birds and butterflies. These results suggest that there is no distinct time lagged response of hose species to the spatial-temporal changes in the landscape. A stronger relationship was evidenced when the variable of 2007 was added to the plant models, suggesting a time lagged response of plants to temporal landscape variability around this year which may be due to a change in agricultural practices and a switch in crop types during that tiem in the study region. This finding is in agreement with that of Krauss et al. (2010) for plants as they found a time lagged response for wild vascular plants, and butterflies which they did not find a distinct lag effect. Also contrary to this study, Findlay and Bourdages (2000), Dullinger et al. (2012) and Sales et al. (2015) found evidence of time lagged response for birds, mammals and herptiles to landscape metrics.

\subsubsection{Limitations and recommendations for future research}

The low spatial resolution of MODIS was one of the main limitations of this study, particularly for use with $1 \times 1 \mathrm{~km}$ landscapes where only 12-16 pixels covered the landscape extent. 
Different fusion techniques are developed to combine MODIS data with high spatial resolution data to overcome the constraint caused by coarse spatial resolution. For example, Vina et al. (2016) used an optimized image fusion technique to combine MODIS and Landsat data and derived land surface phenology to model against floristic diversity. Such a technique is suggested for further research to minimize the limitations of coarse spatial resolution and take advantage of both high temporal and high spatial resolution remote sensing data. In addition, recently launched high spatial/high temporal resolution sensors such as Sentinel-2 with a revisit time of 3 days and spatial resolution of 10,20 and $60 \mathrm{~m}$ can be used in the future to minimize the drawbacks of low spatial resolution and improve the capability of remote sensing data in multi-spatial and multitemporal biodiversity modeling.

Another limitation of this study was the temporally constrained field biodiversity data, which was surveyed only during 2011-2012, while remote sensing data were available for each year back to 2000 . Ideally, a long-term study would include biodiversity data from the same years as the landscape remote sensing variables. This is not always feasible since biodiversity field surveys are time consuming and labour intensive. In addition, when the purpose of the study is to use historic landscape variables, it is not possible to go back in time and collect field data for the past years. One recommendation for future research is designing a long-term systematic biodiversity field survey protocol to monitor the richness and abundance of target species for decadal time spans and model the biodiversity of each year against the corresponding landscape variables of the same year. In addition, available long-term biodiversity databases such as the archive of North America Breeding Bird Survey (BBS) are recommended for future research to create models of biodiversity against explanatory variables of same year.

Overall, the strong relationships of the MODIS derived phenological variables with biodiversity, especially at larger spatial extents was promising, however, further investigation is 
needed to test these relationships in different agricultural environments, or in other environments such as forests, wetlands and arctic tundra.

\subsection{Conclusions}

This study demonstrated that vegetation phenology and dynamics in agricultural lands are related to farmland biodiversity for certain taxa such as plants and beetles. It emphasized the potential of MODIS weekly NDVI datasets and MODIS-derived phenological metrics, especially the time of start of season and vegetation productivity at the time of start of season in modeling the response of farmland species. No evidence of time lagged response of species diversity to past phenological changes in the landscape at MODIS spatial resolution was found. 


\section{Chapter 5: Modeling the relationship between long-term landscape dynamics and local biodiversity of agricultural lands in Eastern Ontario using Landsat time-series}

\subsection{Introduction}

Monitoring the impacts of habitat loss and habitat fragmentation, land transformation and climate change on biodiversity requires long-term global data collection and decadal time series data acquisition. Continuous long-term Earth observation information is only available from a few satellite systems (Turner et al., 2015). The Landsat program operated by the U.S Geological Survey (USGS) and the National Aeronautics and Space Administration (NASA) since 1972 provides one of the longest archives of global coverage. After the opening of the Landsat archive for free access in 2008, a very significant increase in use of Landsat data for time series analysis and change detection studies occurred. In addition, the development of the Landsat Ecosystem Disturbance Adaptive Processing System (LEDAPS) software has made atmospheric correction for Landsat fully automated. The free distribution of the LEDAPS Landsat surface reflectance product has increased the popularity of Landsat for ecological time series analysis and change detection studies (Zhu et al., 2017).

Landsat time series have long been used for ecosystem monitoring and habitat change detection. In addition, metrics extracted from Landsat time-series have been widely used for biodiversity monitoring and especially biodiversity - landscape heterogeneity modelling. Examples of such studies are presented in 2.3.1.1. These metrics have been derived from discrete land cover maps derived through classification or from continuous spectral band (reflectance) data. Various methods of extracting continuous metrics from spectral bands including spectral band combinations (such as vegetation indices) and band transformation techniques (such as TCT) were discussed Chapter 2.3.1.1 and examples of studies which used Landsat vegetation 
idices and TCT for vegetation monitoring was presented and the capability of Landsat-based continuous metrics to predict biodiversity has been compared to that of discrete land cover classes (Duro, et al., 2014; Nguyen et al., 2016; Misurec et al., 2016).

This study hypothesizes that continuous metrics of mid growing season vegetation productivity and moisture such as vegetation indices and TCT derived from long-term interannual time-series of Landsat data are related to farmland biodiversity in a mixed agricultural landscape. It was expected that decadal changes in the landscape affect the diversity of farmland species such as birds, butterflies, beetles, spiders, bees and plants and that these changes could be detected using such spectral indices.

\subsection{Research objective and questions}

The overall objective of this study was to determine if long-term inter-annual mid-summer vegetation dynamics and the variability in crop productivity, vegetation, soil moisture and overall landscape brightness are related to biodiversity in farmlands. The research questions were:

1. Are mid-summer crop productivity, vegetation/soil (i.e., landscape) moisture and overall landscape brightness for individual years from 1985 to 2011 related to farmland biodiversity measured in 2011 and 2012 ? i.e., Is there a lag effect in the response of biodiversity to these landscape characteristics?

2. Are these landscape-biodiversity relationships stronger for larger landscape spatial extents (3 x $3 \mathrm{~km}$ vs. 1 x $1 \mathrm{~km})$ ?

3. Of the seven taxa assessed, do certain taxa show consistently stronger relationships with midsummer crop productivity, landscape moisture or brightness, and what are the most important remotely-sensed variables related to biodiversity of these taxa? 


\subsection{Data}

The study area, landscapes and biodiversity data were described in Chapter 3.1 and 3.2.1. Sixteen Landsat 5 Thematic Mapper (TM) Level-1 Surface Reflectance scenes (row 29/path 15) from 1985 to 2011 were downloaded. The data had been atmospherically corrected using LEDAPS, as described in Appendix II. Landsat 5 TM surface reflectance data are available from March 1984 to November 2011. In this study data that had less than 5\% cloud cover were used. This threshold was selected because a higher percentage of cloud may have covered a significant portion of the study region and landscapes, thereby reducing the numbers of sample landscapes in those years with higher cloud cover and resulting in inconsistent sample sizes for different years.

The dates (Table 5.1) of Landsat acquisition were selected to be within the expected range of the mid-summer peak of the growing season (July to mid-August). However, this was not always possible and two scenes were outside this range by a small amount (1987 - August 20 and 1996 - August 28). Mid-summer cloud free data for years 1986, 1999, 2000, 2002 and 2004 as well as between 1987 to 1994 were not available. Since the Landsat 5 mission ended in November 2011, Landsat 5 data for 2012 were not available. Landsat 7 was not used in this study due to the striping issue (USGS, 2019) and the variation in TM and ETM+ sensor radiometric properties.

To ensure that variability in the Landsat data acquisition dates would not cause an issue in further temporal analysis, seasonal NDVI values were analyzed using Weekly MODIS NDVI data (employed in Chapter 4). Weekly NDVI curves demonstrated that the vegetation biomass reached its peak around early July and these NDVI values remained relatively steady during July and August. The range of NDVI values from early July to mid/end of August was less than 0.04 for all years suggesting that Landsat images of this period could be used to represent mid-summer green vegetation biomass and productivity as well as soil brightness and moisture content and other landscape parameters. 
Table 5.1. Year and date of the selected Landsat 5 TM data

\begin{tabular}{|r|c|c|r|r|c|}
\hline$\#$ & Year & Date & $\#$ & Year & Date \\
\hline 1 & 1985 & 13-Jul & 9 & 2003 & 15-Jul \\
\hline 2 & 1987 & 20 Aug & 10 & 2005 & 20-Jul \\
\hline 3 & 1994 & 07-Aug & 11 & 2006 & 07-Jul \\
\hline 4 & 1995 & 10-Aug & 12 & 2007 & 26-Jul \\
\hline 5 & 1996 & 28-Aug & 13 & 2008 & 12-Jul \\
\hline 6 & 1997 & 30-Jul & 14 & 2009 & 15-Jul \\
\hline 7 & 1998 & 02-Aug & 15 & 2010 & 02-Jul \\
\hline 8 & 2001 & 10-Aug & 16 & 2011 & 05-Jul \\
\hline
\end{tabular}

In addition, because Table 5.1 shows that images were most commonly acquired in August for the period 1985-2001 and in July between 2003-2011, the mean of NDVI over all pixels and landscapes was used in a SWR model against the year and day of year of data acquisition to test the potential effect of acquisition timing on NDVI. Neither variable entered the model at $\mathrm{p}=0.05$ indicating that the acquisition date or year over those two months had no effect on NDVI values $(\mathrm{R}=0.3, \mathrm{p}=0.16)$. Hence, Landsat images for both periods were used to represent mid-summer green vegetation biomass and productivity.

\subsection{Methods}

Figure 5.1 outlines the main processes and methods used in this chapter. A detailed description of each step follows. 


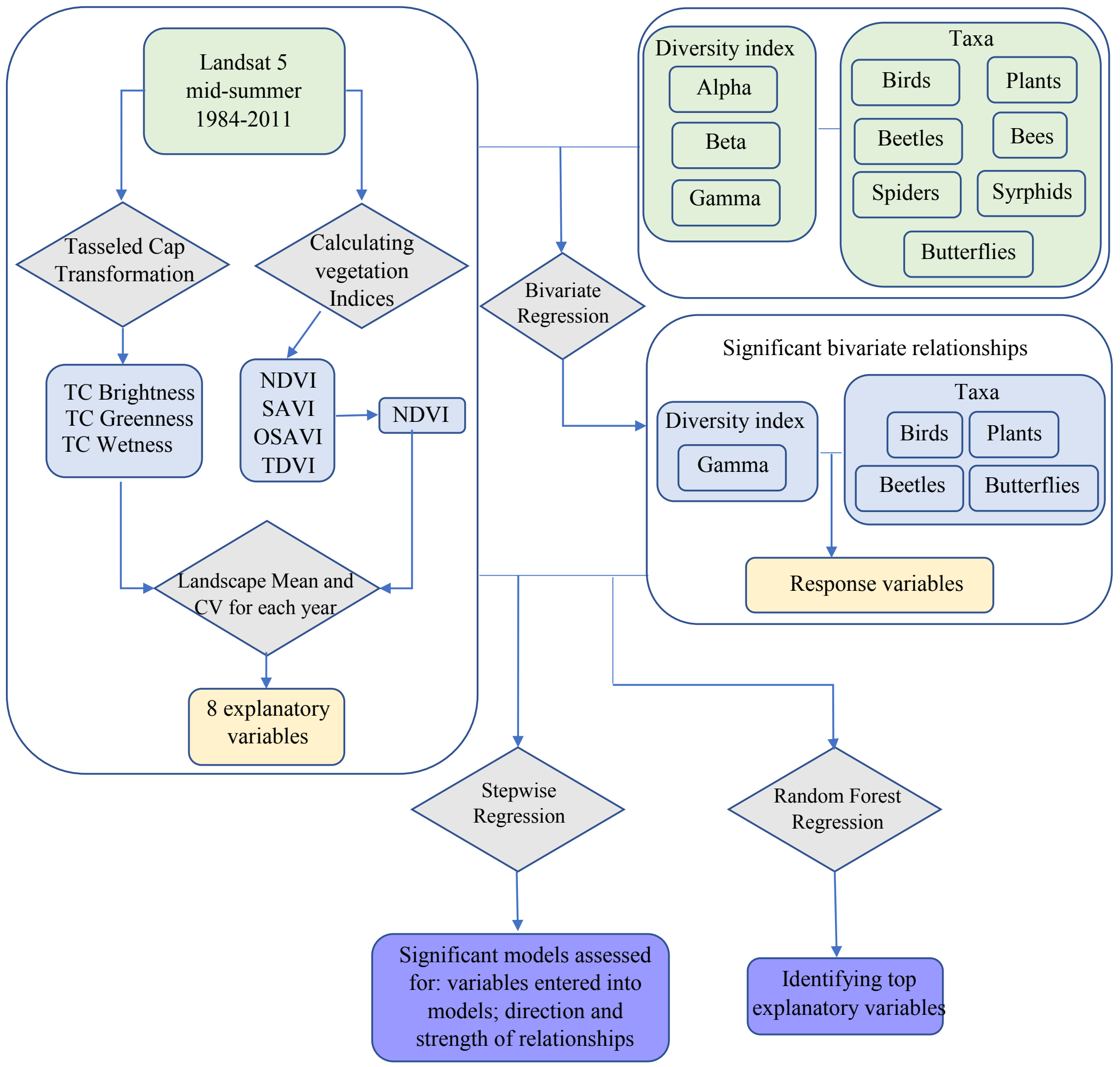

Figure 5. 1. Flow chart outlining various steps used in this chapter. This includes the extraction of TCT and vegetation indices from Landsat 5 archive and selection of response variables, as well as the statistical modeling to assess the most important variables and the direction and magnitude of relations with gamma diversity. 


\subsubsection{Vegetation indices}

In this study, four vegetation indices (VIs) were calculated using the Landsat 5 TM spectral bands as described below. They were selected because they have been broadly used in different environments, especially for low to medium density vegetation such as agricultural lands, and they have provided valuable insight into various vegetation parameters (Examples: Panda et al., 2010; Hatfield and Prueger, 2010; Duro et al., 2014; Qi et al., 2017). The Normalized Difference Vegetation Index (NDVI, Tucker, 1979) was selected because it has become a standard often utilized for comparison with other VIs. However, in crops or semi-arid environments, NDVI has been shown to be affected by background soil reflectance, which is generally greater in the red and less in the NIR than vegetation (Huete, 1988). The Soil Adjusted Vegetation Index (SAVI, Huete, 1988) was developed to adjust the soil effect and improve NDVI by increasing its sensitivity to the soil colour, brightness, and moisture and to minimize saturation effects from high density vegetation. The Optimized Soil Adjusted Vegetation Index (OSAVI, Rondeaux et al., 1996) was developed for agricultural monitoring and includes an optimized soil adjustment coefficient. It is robust to variability in soil brightness and is best used in areas with relatively sparse vegetation where soil is visible through the canopy. The Transformed Difference Vegetation Index (TDVI, Bannari et al., 2002) was developed to improve on SAVI by minimizing the artifacts caused by the optical properties of bare soil and to improve on NDVI with respect to the saturation problem in high density vegetation. NDVI was defined in Chapter 2.3.1 and the formulation was given in Equation 2.1. Formulations for SAVI, OSAVI and TDVI are given as Equations 5.1 to 5.3, respectively:

$S A V I=\frac{(1+\mathrm{L}) \times(\mathrm{NIR}-\mathrm{RED})}{(\mathrm{NIR}+\mathrm{RED}+\mathrm{L})}$

(Equation 5.1)

$O S A V I=\frac{(\mathrm{NIR}-\mathrm{RED})}{(\mathrm{NIR}+\mathrm{RED}+0.16)}$

(Equation 5.2) 
$T D V I=\frac{(1+\mathrm{L}) \times(\mathrm{NIR}-\mathrm{RED})}{\sqrt{N I R^{2}+\mathrm{RED}+\mathrm{L}}}$

(Equation 5.3)

where $\mathrm{L}$ is a soil brightness correction factor that varies depending on the amount or cover of green vegetation (high density vegetation, $L=0$; no green vegetation, $L=1$ ). The default $L$ value of 0.5 , which works well in moderate density vegetation covers such as agricultural fields in mid-summer (Huete, 1988; Bannari et al., 2002), was used.

The selected vegetation indices were calculated and mapped over the landscapes for all years independently and they were analyzed and compared to determine if they represented gradients of vegetation density evident in the images and with reference to air photos. In addition, the mean of these vegetation indices was calculated across the sample landscapes and correlation analysis was conducted between them to determine the vegetation index that best represents the variability in the vegetation density across landscapes and demonstrates unique information to justify its use in subsequent biodiversity modeling.

\subsubsection{Tasseled Cap components}

The Tasseled Cap Transformation (TCT) compresses highly correlated spectral bands into fewer decorrelated variables by transforming them into a new set of orthogonal axes (Equation 5.4) associated with physical features and gradients representing Brightness, Greenness and Wetness as explained in 2.3.1.2. TCT components have largely been used for change detection and trend analysis (Nguyen et al., 2016; Misurec et al., 2016; presented in Chapter 2.3.3.1.1) and were introduced as measures of spectral heterogeneity to model against biodiversity (Rocchini et al., 2010). Thus, they were selected in this study to portray the spatial and temporal variability in landscape brightness, productivity and moisture content and their relationships with farmland biodiversity. The band coefficients are listed in Table 5.2 (Crist, 1985). 
TCT Component $=\rho B 1 \times C_{-} B 1+\rho B 2 \times C_{-} B 2+\rho B 3 \times C_{-} B 3+\rho B 4 \times C_{-} B 4+\rho B 5 \times C_{-} B 5+$ $\rho B 7 \times C_{-} B 7 \quad$ (Equation 5.4)

Table 5.2. TM bands coefficients for each TCT component

\begin{tabular}{ccccccc}
\hline Component & $\mathrm{B} 1$ & $\mathrm{~B} 2$ & $\mathrm{~B} 3$ & $\mathrm{~B} 4$ & $\mathrm{~B} 5$ & $\mathrm{~B} 7$ \\
\hline Brightness & 0.2043 & 0.4158 & 0.5524 & 0.5741 & 0.3124 & 0.2303 \\
Greenness & -0.1603 & -0.2819 & -0.4934 & 0.7940 & -0.0002 & -0.1446 \\
Wetness & 0.0315 & 0.202 & 0.3102 & 0.1594 & -0.6806 & -0.7078 \\
\hline
\end{tabular}

The TCT was calculated in ArcGIS 10.4 where the output Brightness (TCB), Greenness (TCG) and Wetness (TCG) components were created in 8-bit raster format with 0-255 value range. The mean and coefficient of variation (CV) for these components and the four VIs were calculated for each sample landscape independently for each year. They were then input as explanatory variables in diversity models for each taxon. To test the relative effect of sample landscape size on the models, all variables were extracted for $1 \times 1 \mathrm{~km}$ and $3 \times 3 \mathrm{~km}$ sample landscapes independently.

\subsubsection{Statistical modeling}

Statistical modelling followed the same steps as given in the MODIS phenology study of Chapter 4. Initial exploratory modeling was conducted using a bivariate regression model to determine and compare potential relationships between alpha, beta and gamma diversity for the seven surveyed taxa and the mean and CV of NDVI (the selected VI; see Section 5.6.1) and the TCT components. The goal was to select the diversity index and taxa that demonstrated consistently significant relationships with the remote sensing variables for use in subsequent RFR and SWR analyses. 
However, in contrast to Chapter 4, since the number of explanatory variables was less than $1 / 10$ of the number of observations (93), all eight explanatory variables were input in both RFR (to determine relative variable importance) and SWR (to determine model significance and strength, the explanatory variables entered, and the direction of their relationship with biodiversity) to compare and contrast the results of two modeling techniques. Details of RFR and SWR modelling procedures were described in Chapter 4.5.3.1. These two techniques were used in this study based on their similar results to the alternative statistical methods (AICc and LMG) tested in Chapter 4. Potential for a lag effect in landscape summer vegetation productivity, moisture or brightness on biodiversity was also tested as explained in Section 4.5.3.1.5. Models which were created to test the lag effect are listed in Table 5.3.

Table 5. 3. List of models with best explanatory variable of each year to test time lagged response of species to phenological changes

\begin{tabular}{|l|l|}
\hline Models & Input explanatory variables \\
\hline M1 & $V_{2011}$ \\
\hline M2 & $V_{2011}+V_{2010}$ \\
\hline M3 & $V_{2011}+V_{2010}+V_{2009}$ \\
\hline M $~$ & $V_{2011}+V_{2010}+V_{2009}+V_{2008}$ \\
\hline M5 & $V_{2011}+V_{2010}+V_{2009}+V_{2008}+V_{2007}$ \\
\hline M6 & $V_{2011}+V_{2010}+V_{2009}+V_{2008}+V_{2007}+V_{2006}$ \\
\hline M7 & $V_{2011}+V_{2010}+V_{2009}+V_{2008}+V_{2007}+V_{2006}+V_{2005}$ \\
\hline M8 & $V_{2011}+V_{2010}+V_{2009}+V_{2008}+V_{2007}+V_{2006}+V_{2005}+V_{2003}$ \\
\hline M9 & $V_{2011}+V_{2010}+V_{2009}+V_{2008}+V_{2007}+V_{2006}+V_{2005}+V_{2003}+V_{2001}$ \\
\hline M10 & $V_{2011}+V_{2010}+V_{2009}+V_{2008}+V_{2007}+V_{2006}+V_{2005}+V_{2003}+V_{2001}+V_{1998}$ \\
\hline M11 & $V_{2011}+V_{2010}+V_{2009}+V_{2008}+V_{2007}+V_{2006}+V_{2005}+V_{2003}+V_{2001}+V_{1998}+V_{1997}$ \\
\hline M12 & $V_{2011}+V_{2010}+V_{2009}+V_{2008}+V_{2007}+V_{2006}+V_{2005}+V_{2003}+V_{2001}+V_{1998}+V_{1997}+V_{1996}$ \\
\hline M13 & $V_{2011}+V_{2010}+V_{2009}+V_{2008}+V_{2007}+V_{2006}+V_{2005}+V_{2003}+V_{2001}+V_{1998}+V_{1997}+V_{1996}+V_{1995}$ \\
\hline M14 & $V_{2011}+V_{2010}+V_{2009}+V_{2008}+V_{2007}+V_{2006}+V_{2005}+V_{2003}+V_{2001}+V_{1998}+V_{1997}+V_{1996}+V_{1995}+V_{1994}$ \\
\hline M15 & $V_{2011}+V_{2010}+V_{2009}+V_{2008}+V_{2007}+V_{2006}+V_{2005}+V_{2003}+V_{2001}+V_{1998}+V_{1997}+V_{1996}+V_{1995}+V_{1994}+V_{1987}$ \\
\hline M16 & $V_{2011}+V_{2010}+V_{2009}+V_{2008}+V_{2007}+V_{2006}+V_{2005}+V_{2003}+V_{2001}+V_{1998}+V_{1997}+V_{1996}+V_{1995}+V_{1994}+V_{1987}+V_{1985}$ \\
\hline
\end{tabular}




\subsection{Results}

\subsubsection{Vegetation indices}

Strong positive correlations between NDVI, SAVI, OSAVI and TDVI were found for all years and both landscape extents. Table 5.4 presents example results for 2011 Landsat data.

Table 5.4. Pearson correlation between the landscape mean of the four vegetation indices derived from 2011 Landsat data for $3 \times 3 \mathrm{~km}$ landscapes. $\mathrm{P} \leq 0.05$ for all correlations.

\begin{tabular}{|c|c|c|c|c|}
\hline & NDVI & OSAVI & SAVI & TDVI \\
\hline NDVI & 1 & & & \\
\hline OSAVI & $\mathbf{0 . 9 9}$ & 1 & & \\
\hline SAVI & $\mathbf{0 . 9 9}$ & $\mathbf{0 . 9 4}$ & 1 & \\
\hline TDVI & $\mathbf{0 . 9 4}$ & $\mathbf{0 . 9 4}$ & $\mathbf{0 . 9 4}$ & 1 \\
\hline
\end{tabular}

Figure 5.3 shows the NDVI map of the study area in 2011 as an example. Land surface NDVI values were between 0 and 1 , while water pixels had values of zero or negative values. Low NDVI values between 0.2 to 0.4 (orange colour) indicate short vegetation and a mixture of soil radiance and vegetation radiance within the $30 \mathrm{~m} \times 30 \mathrm{~m}$ pixels. However, the strong relationship of NDVI with SAVI and OSAVI demonstrates that NDVI was not adversely affected by such soil radiance. These three vegetation indices essentially provide equivalent information and there was no significant advantage of the soil adjusted indices over NDVI. Figure 5.4 shows NDVI and TDVI for a sample landscape. Although the landscapes were comprised of predominantly agriculture land cover, TDVI displayed large areas of saturation as $34.7 \%$ of all pixels within the total area of $3 \times 3 \mathrm{~km}$ sample landscapes displayed values of 1.0 (examples illustrated by the ellipses in Figure 5.3). Such saturation was not evident in NDVI values. TDVI appears to be less sensitive to variability in crop vegetation density. Given the above results comparing the four vegetation indices, it was determined that NDVI best captured the withinfield and between-field vegetation density and biomass. It was therefore retained for use in biodiversity modelling while the soil adjusted indices were discarded. 


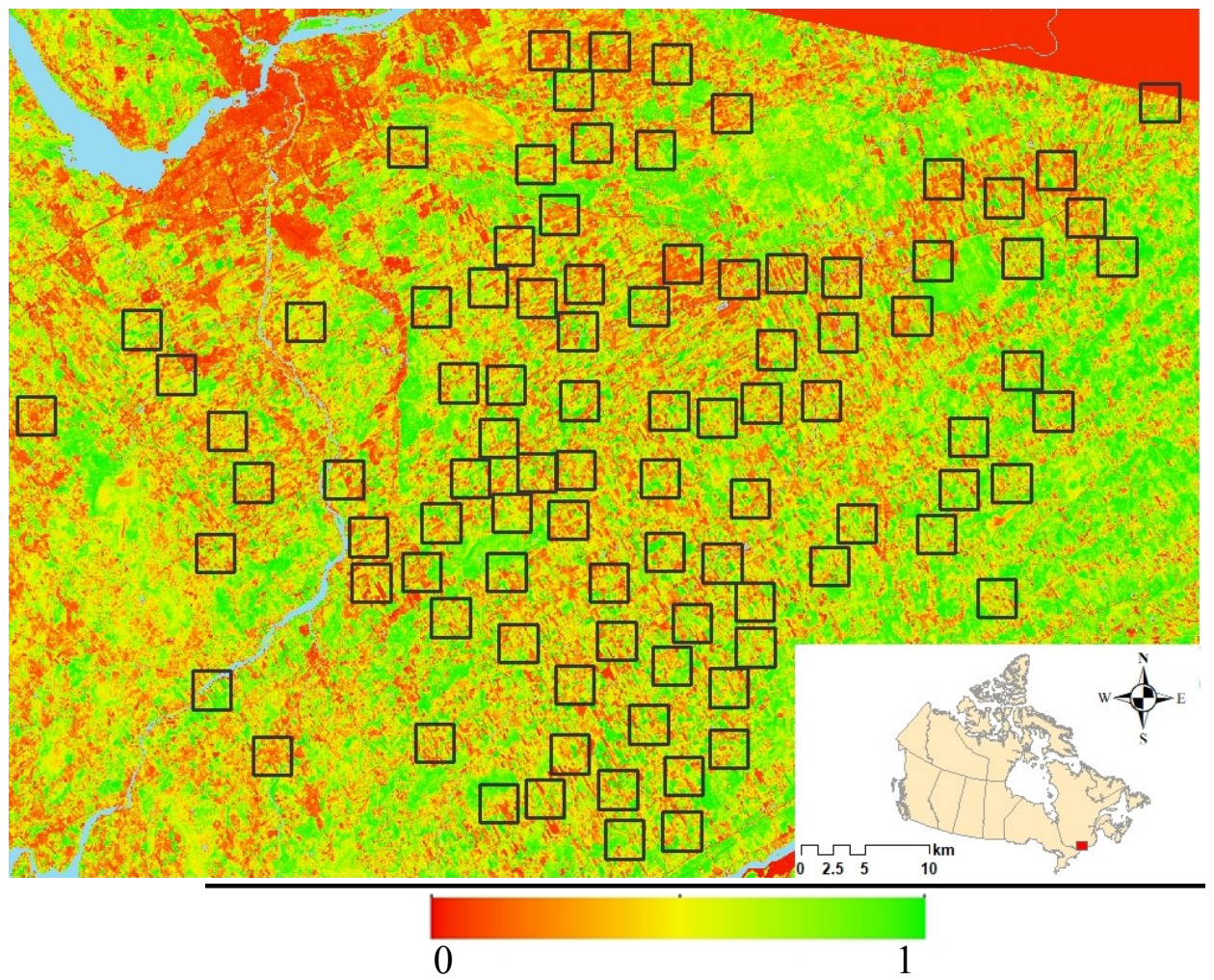

Figure 5. 2. Example NDVI map of the study area for 2011 Landsat data. Land surface NDVI values were $>0$, while water pixels were $\leq 0$.

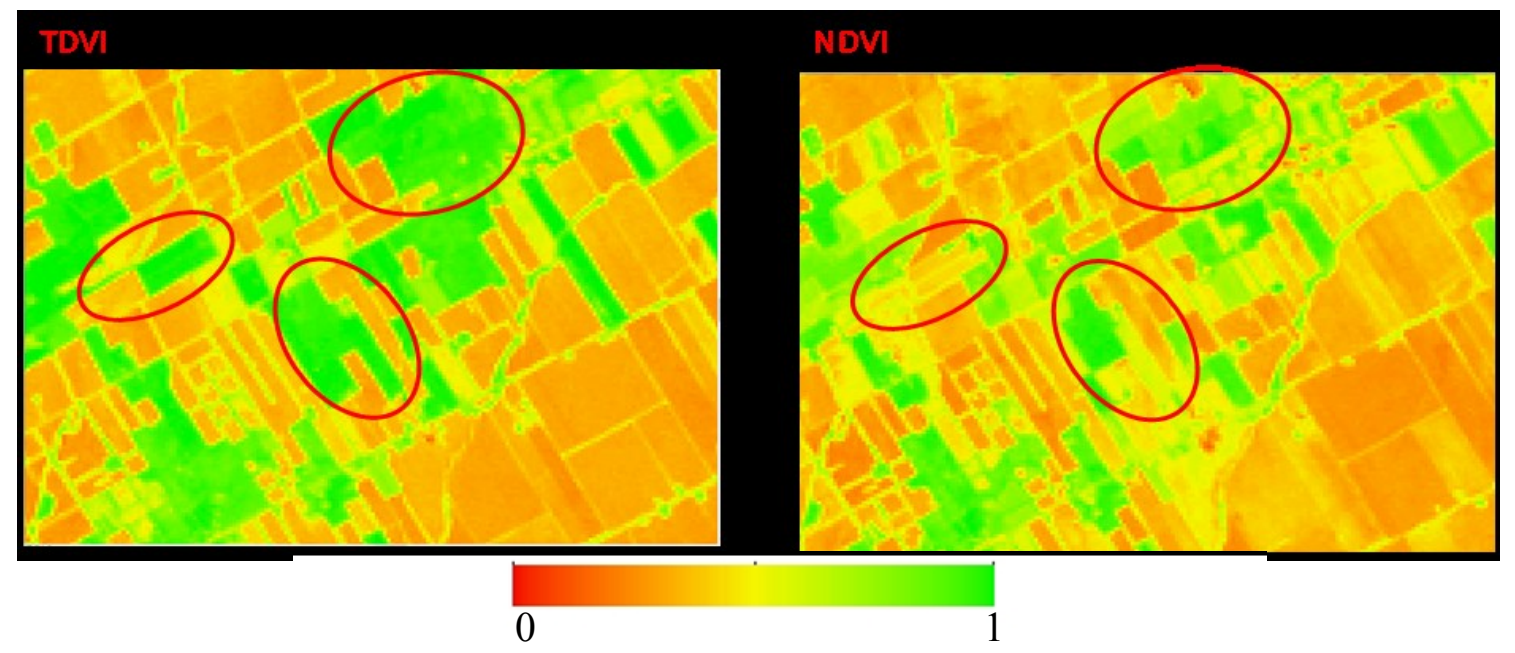

Figure 5.3. Comparing the sensitivity of NDVI and TDVI (derived from 2011 Landsat data as an example) in capturing the within-field and between-field variability in vegetation density. Red ellipses delineate example areas of higher density vegetation at which TDVI tends to saturate. 


\subsubsection{TCT components}

TCB, TCG and TCW, were calculated for each year individually. Figure 5.5 shows the images of TCT components for 1985.

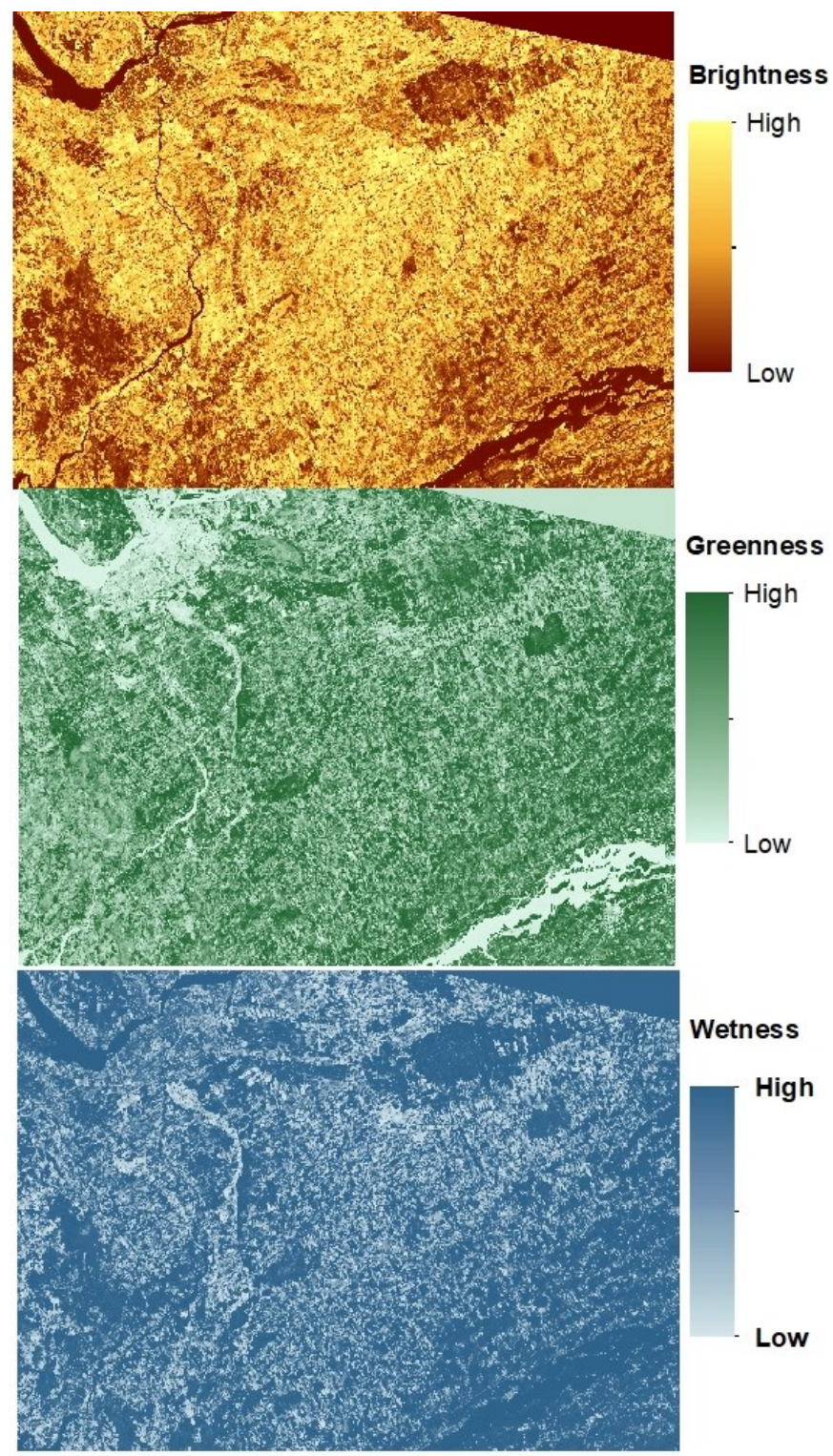

Figure 5.4. TCB, TCG and TCW images of the study region, 1985

TCB was associated with bare soil and human made features such as roads and buildings

(Figure 5.6 a). High-density croplands and forest patches were associated with high wetness in 
most areas (Figure $5.6 \mathrm{~b}$ and c, respectively), while many high brightness and low greenness areas, such as bare soil and urban features, are associated with low wetness (Figure 5.6 a).

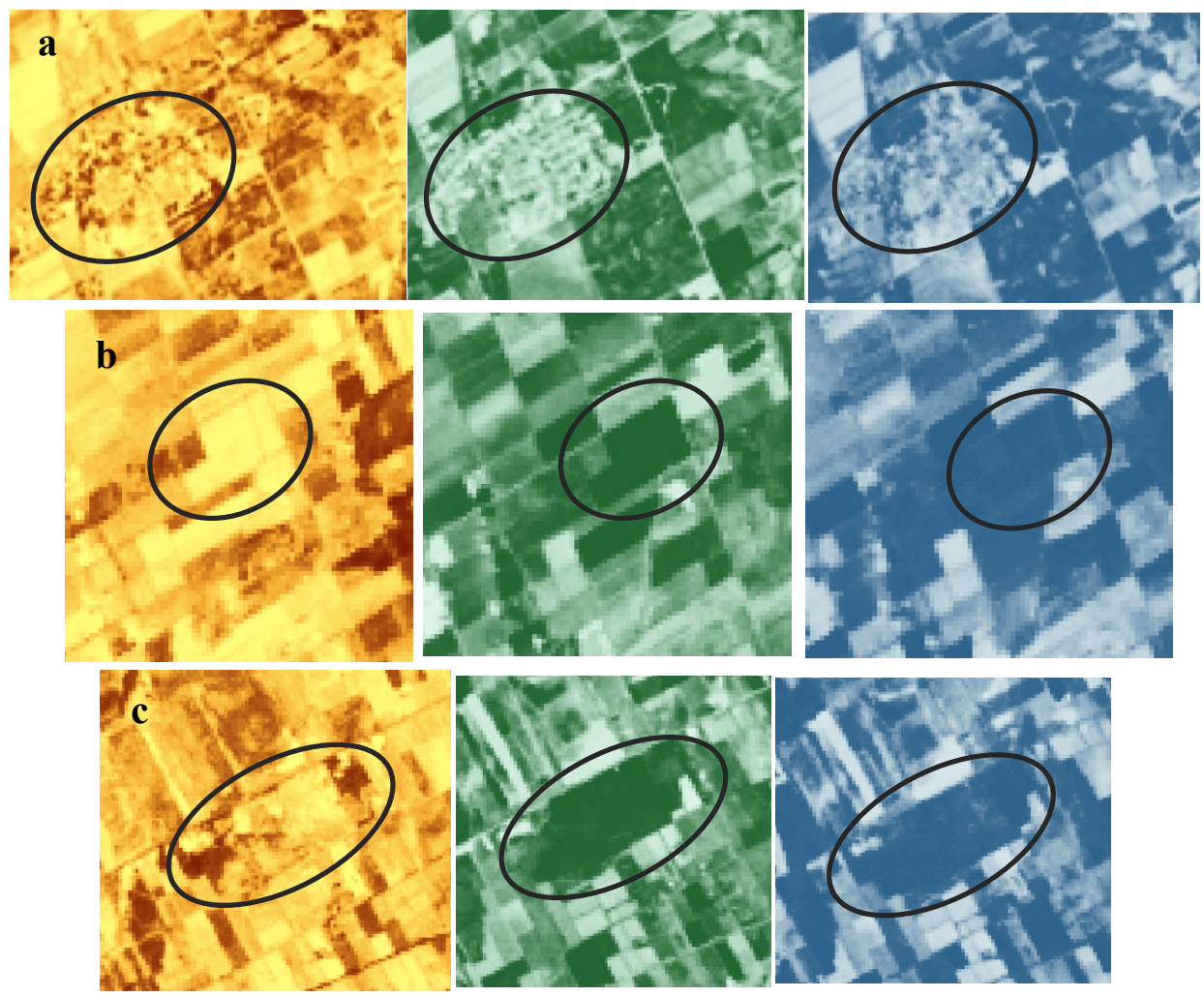

Figure 5.5. Comparing the spatial association of 1985 Landsat TCB (shown in yellow), TCG (shown in green) and TCW (shown in blue) in a) high density vegetation croplands, b) wooded areas, and c) buildings.

\subsubsection{Statistical modeling}

\subsubsection{Exploratory modeling}

Bivariate regression modeling of the eight remote sensing variables (mean and CV of NDVI, TCB, TCG, and TCW) against alpha, beta and gamma diversity for all taxa and years showed that plants and beetles demonstrated the strongest relationships in most years $(|r| \leq 0.45)$, with many models being significant $(\mathrm{p} \leq 0.05)$, while bees, syrphids and spiders did not demonstrate significant responses $(|\mathrm{r}| \leq 0.20 ; \mathrm{p}>0.05)$. Therefore, as for the MODIS phenology analysis of Chapter 4, bees, syrphids and spiders were eliminated from further modelling. Models of varying strength were produced for bird and butterfly diversity for the different years of Landsat data. 
Both were retained for further modelling using RFR and SWR for the same reasons given in Chapter 4.

In addition, the relationships between $\% \mathrm{Ag}$ and the mean of the explanatory variables were found to be strong ( $\mathrm{r} \geq 0.68$, Table 5.5). Higher $\% \mathrm{Ag}$ in a sample landscape is associated with lower natural and semi-natural land covers and hence higher amounts of bare soil and short vegetation. Increased agriculture therefore resulted in decreased Mean TCG, TCW and NDVI or decreased vegetation biomass and productivity. These strong relationships suggested that, as for some of the phenological variables in Chapter 4, \% Ag was manifested in the remote sensing variables and it did not need to be entered into the models as a explanatory variable. On the other hand, the pixel to pixel variability $(\mathrm{CV})$ of the predictors was not related to \% Ag. Spatial variability in vegetation productivity and the amount of bare soil and moisture over a landscape may be higher in more diverse landscapes with smaller fields, while such landscapes may have the same amount of cultivated area as less diverse landscapes with larger fields (same \% $\mathrm{Ag}$ ).

Table 5.5. R-values between $\% \mathrm{Ag}$ and $3 \times 3 \mathrm{~km}$ explanatory variables in 2011. Significant relationships $(\mathrm{p} \leq 0.05)$ are shown in bold.

\begin{tabular}{ccccccccc}
\hline & MEAN TCB & TCB CV & MEAN TCG & TCG CV & MEAN TCW & TCW CV & MEAN NDVI & NDVI CV \\
\hline$\% \mathrm{Ag}$ & $\mathbf{0 . 6 8}$ & 0.07 & $\mathbf{- 0 . 8 0}$ & 0.08 & $\mathbf{- 0 . 8 0}$ & 0.08 & $\mathbf{- 0 . 8 1}$ & 0.59 \\
\hline
\end{tabular}


In comparing bivariate models of alpha, beta and gamma diversity against explanatory variables, alpha generally produced weaker, and in many cases, insignificant models for all taxa in most years. Gamma and beta generally produced stronger models. However, the strength of gamma and beta models was inconsistent and the significance varied between predictors, taxa and years. Figure 5.7 presents an example of strong bivariate models between beetle diversity indices and Mean TCB derived for $3 \times 3 \mathrm{~km}$ landscapes and 1994 Landsat data. In this example, all diversity indices produced significant models but beta and gamma were stronger $(r=0.41$ and $r$ $=0.4$, respectively). Gamma was selected instead of beta for further modelling as a representative of overall diversity for the same reasons given in Chapter 4.5.2.1.
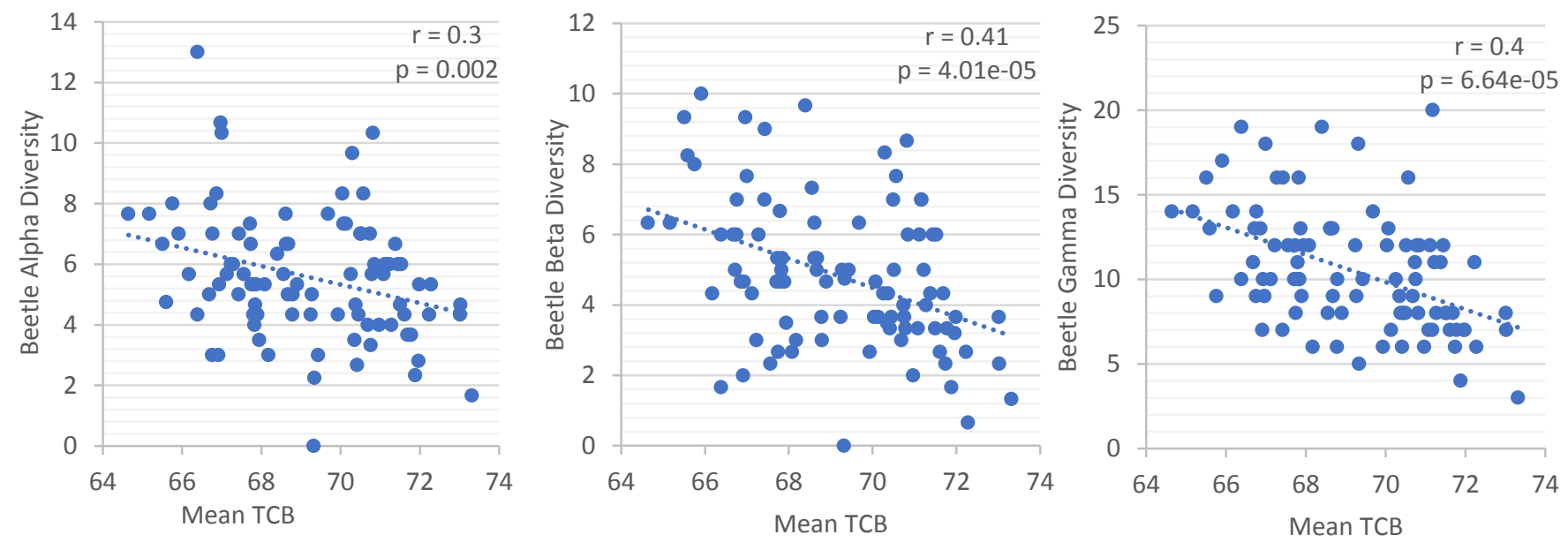

Figure 5.6. Comparing the relationship of alpha, beta and gamma diversity of beetles with Mean TCB derived from 1994 Landsat data for 3 x $3 \mathrm{~km}$ landscapes.

The bivariate correlations between the explanatory variables were determined for each year independently and were all moderate to low. TCG CV and Mean TCB showed the lowest correlation $(\mathrm{r}=0.02)$, while Mean NDVI and Mean TCG showed the highest correlation $(\mathrm{r}=$ 0.74). The Pearson correlation matrix for all explanatory variables for $3 \times 3 \mathrm{~km}$ sample landscapes in 2011 is presented in Table 5.7. High correlation between variables has been found to affect the accuracy of RF classification (Millard and Richardson, 2015), but the betweenvariables correlation found here $\left(r \leq 0.74\right.$, or $\left.r^{2}=0.41\right)$ was lower than the $r$-value threshold 
introduced in various sources $(r<0.85$ Behnamian et al., in press) and was not considered of significant concern.

Table 5.6. Pearson correlation matrix for all explanatory variables in the $3 \times 3 \mathrm{~km}$ landscape models for 2011. Correlations significant at $\mathrm{p} \leq 0.05$ are in bold.

\begin{tabular}{|c|c|c|c|c|c|c|c|c|}
\hline & $\begin{array}{c}\text { MEAN } \\
T C B\end{array}$ & $T C B C V$ & $\begin{array}{c}\text { MEAN } \\
\text { TCG }\end{array}$ & TCG CV & $\begin{array}{c}\text { MEAN } \\
\text { TCW }\end{array}$ & TCW CV & $\begin{array}{c}\text { MEAN } \\
\text { NDVI }\end{array}$ & $\begin{array}{c}N D V I \\
C V\end{array}$ \\
\hline MEAN TCB & 1 & & & & & & & \\
\hline TCB CV & -0.66 & 1 & & & & & & \\
\hline MEAN TCG & 0.27 & -0.31 & 1 & & & & & \\
\hline TCG CV & 0.02 & 0.25 & -0.18 & 1 & & & & \\
\hline MEAN TCW & -0.46 & 0.18 & 0.33 & -0.20 & 1 & & & \\
\hline TCW CV & 0.43 & -0.09 & -0.20 & 0.44 & -0.58 & 1 & & \\
\hline MEAN NDVI & 0.55 & -0.63 & 0.74 & -0.35 & 0.20 & -0.15 & 1 & \\
\hline NDVI CV & -0.66 & 0.68 & -0.41 & 0.47 & 0.03 & 0.06 & -0.61 & 1 \\
\hline
\end{tabular}

\subsubsection{Random forest biodiversity modelling}

RFR was run for the selected taxa using Landsat derived variables for all years independently as described in Chapter 4.5.3.1, except for 2012 due Landsat-5 having been terminated. Table 5.8 summarizes the model error measures by taxa for the 2011 Landsat variables and $1 \times 1 \mathrm{~km}$ and 3 $\times 3 \mathrm{~km}$ landscape extents. Model errors of all years (1985-2011) are listed in Appendix IV. The variability accounted for (\% Var) is negative for all models, which indicates that the Landsat variables did not explain the variance of the training set; i.e., the out-of-bag gamma predictions had larger variance than the variance of the training data (Liaw and Wiener, 2002). \% Var was higher (less negative) for the $1 \times 1 \mathrm{~km}$ plants model but lower (more negative) for $1 \times 1 \mathrm{~km}$ beetles, butterflies and birds models compared to $3 \times 3 \mathrm{~km}$ models. The MSR, as an indicator of the difference between the observed and predicted gamma values, was highest for plants, especially with respect to the number of plant species (227), while it was much lower than the number of beetle (80), bird (52) and butterfly (30) species. The MSR values were encouraging as they were below the range of gamma $(80.65 \%$ of the range for beetles $(20), 54.2 \%$ of the range for birds (14), and 59.9\% of the range for butterflies (16)), compared to the MSR for plants which 
was larger than the range of gamma (68). Given these MSR results, it was decided to continue with variable importance analysis in RFR models and SWR analysis.

Table 5.7. Error measures from the random forest regressions summarized by taxon for 2011 . The percent variance (\% Var) and mean of squared residuals (MSR) were calculated on one third of the samples in each tree created in the RFR runs for $1 \times 1 \mathrm{~km}$ to $3 \times 3 \mathrm{~km}$ models independently.

\begin{tabular}{|c|c|c|c|c|c|}
\hline \multicolumn{2}{|c|}{$1 \mathrm{~km}$} & Plants & Beetles & Birds & Butterflies \\
\hline \multirow[b]{3}{*}{ MSR } & Mean & 239.33 & 16.13 & 7.52 & 9.59 \\
\hline & Min & 233.25 & 15.83 & 7.36 & 9.36 \\
\hline & Max & 246.03 & 16.38 & 7.73 & 9.84 \\
\hline \multirow[b]{3}{*}{$\% \operatorname{Var}$} & Mean & -4.75 & -17.36 & -12.85 & -9.81 \\
\hline & Min & -7.67 & -19.21 & -16.02 & -12.62 \\
\hline & Max & -2.08 & -15.22 & -10.4 & -7.14 \\
\hline \multicolumn{2}{|c|}{$3 \mathrm{~km}$} & Plants & Beetles & Birds & Butterflies \\
\hline \multirow[b]{3}{*}{ MSR } & Mean & 248.20 & 14.90 & 7.34 & 10.10 \\
\hline & Min & 243.68 & 14.63 & 7.09 & 9.812 \\
\hline & Max & 254.21 & 15.218 & 14.89 & 14.89 \\
\hline \multirow[b]{3}{*}{$\% \operatorname{Var}$} & Mean & -8.62 & -8.43 & -9.01 & -15.08 \\
\hline & Min & -11.26 & -10.72 & -11.24 & -17.17 \\
\hline & Max & -6.64 & -6.44 & -6.35 & -12.26 \\
\hline
\end{tabular}

Relative variable importance from RFR is given in Table 5.9. For beetles and $1 \times 1 \mathrm{~km}$ sample landscapes, Mean NDVI appeared as the most important variable, while for $3 \times 3 \mathrm{~km}$ landscapes Mean TCB was most important. This illustrates the importance of vegetation productivity as well as the proportion of bare soil visible to the sensor for beetle diversity. On the other hand, Mean TCW was the second most important variable at both landscape extents, which may be due to differences in habitat wetness preference among beetle species.

For birds, Mean and NDVI CV appeared as the first and second most important variables for both $1 \times 1 \mathrm{~km}$ and $3 \times 3 \mathrm{~km}$ landscapes. This emphasized the importance of overall landscape vegetation productivity and the spatial variability of vegetation productivity on bird diversity. 
Mean NDVI was the most important predictor in butterfly models at both landscape extents and Mean TCG was the second most important in $3 \times 3 \mathrm{~km}$ landscape models, indicating the potential importance of overall landscape vegetation productivity to butterfly diversity. NDVI $\mathrm{CV}$ was the second most important for $1 \times 1 \mathrm{~km}$ landscapes.

For plants in $1 \times 1 \mathrm{~km}$ landscapes, Mean NDVI and Mean TCB were the first and second most important predictors, while in $3 \times 3 \mathrm{~km}$ landscapes Mean TCB and Mean TCG were first and second. Higher soil and landscape brightness as an indicator of more fallow and uncultivated fields and lower density crops can promote wild plant diversity.

Overall, at $1 \times 1 \mathrm{~km}$ sample landscapes, Mean NDVI appeared as the most important variable for beetles, butterflies and plants and the second most important variable for birds, while at $3 \times 3$ km sample landscapes, Mean NDVI appeared as the most significant variable for birds and butterflies. This indicated potential significance of mid-summer landscape vegetation productivity for all species groups at different spatial scales. Mean TCB also appeared as the most significant variable for beetles and plants at $3 \times 3 \mathrm{~km}$ models, which highlighted the potential importance of bare soil or reduced crop cover to the diversity of these taxa groups at this larger landscape extent. 
Table 5.8. Relative importance of explanatory variables as a ranked list of their number of occurrences in RFR models for $1 \times 1 \mathrm{k} \mathrm{m}$ and $3 \times 3 \mathrm{~km}$ landscape extents.

\begin{tabular}{|l|r|r|l|r|r|}
\hline \multicolumn{7}{|c|}{ Beetles } \\
\hline Variable & \multicolumn{1}{|c|}{$\boldsymbol{\#}$} & $\mathbf{\%}$ & Variable & \multicolumn{1}{|c|}{$\mathbf{~}$} & \multicolumn{1}{l|}{$\%$} \\
\hline MEAN NDVI & 939 & 58.6 & MEAN TCB & 857 & 53.5 \\
\hline MEAN TCW & 884 & 55.2 & MEAN TCW & 856 & 53.5 \\
\hline NDVI CV & 789 & 49.3 & MEAN NDVI & 798 & 49.8 \\
\hline MEAN TCG & 775 & 48.4 & TCG CV & 730 & 45.6 \\
\hline TCW CV & 541 & 33.8 & MEAN TCG & 621 & 38.8 \\
\hline TCG CV & 353 & 22.0 & NDVI CV & 603 & 37.6 \\
\hline MEAN TCB & 313 & 19.5 & TCW CV & 331 & 20.6 \\
\hline TCB CV & 224 & 14.0 & TCB CV & 4 & 0.2 \\
\hline
\end{tabular}

\begin{tabular}{|l|r|l|l|r|r|}
\hline \multicolumn{7}{|c|}{ Birds } \\
\hline Variable & \multicolumn{1}{|c|}{$\#$} & \% & Variable & \multicolumn{1}{c|}{$\#$} & \multicolumn{1}{l|}{$\%$} \\
\hline NDVI CV & 989 & 61.8 & MEAN NDVI & 1208 & 75.5 \\
\hline MEAN NDVI & 924 & 57.7 & NDVI CV & 897 & 56.0 \\
\hline MEAN TCG & 860 & 53.7 & MEAN TCG & 813 & 50.8 \\
\hline TCW CV & 700 & 43.7 & MEAN TCB & 693 & 43.3 \\
\hline MEAN TCB & 565 & 35.3 & MEAN TCW & 437 & 27.3 \\
\hline MEAN TCW & 509 & 31.8 & TCW CV & 383 & 23.9 \\
\hline TCB CV & 257 & 16.0 & TCG CV & 309 & 19.3 \\
\hline TCG CV & 0 & 0 & TCB CV & 60 & 3.7 \\
\hline
\end{tabular}

\begin{tabular}{|l|r|r|l|l|r|}
\hline \multicolumn{7}{|c|}{ Butterflies } \\
\hline Variable & \multicolumn{1}{|c|}{$\#$} & \multicolumn{1}{|c|}{$\%$} & Variable & \multicolumn{1}{l|}{ 3km } & \multicolumn{1}{l|}{$\%$} \\
\hline MEAN NDVI & 1265 & 79.0 & MEAN NDVI & 1184 & 74 \\
\hline NDVI CV & 1031 & 64.4 & MEAN TCG & 835 & 52.1 \\
\hline MEAN TCG & 979 & 61.1 & TCB CV & 672 & 42.0 \\
\hline TCW CV & 775 & 48.4 & MEAN TCW & 588 & 36.7 \\
\hline MEAN TCW & 711 & 44.4 & NDVI CV & 511 & 31.9 \\
\hline MEAN TCB & 31 & 1.9 & TCW CV & 432 & 27 \\
\hline TCB CV & 8 & 0.5 & MEAN TCB & 333 & 20.8 \\
\hline TCG CV & 0 & 0 & TCG CV & 245 & 15.3 \\
\hline
\end{tabular}

\begin{tabular}{|l|r|l|l|r|r|}
\hline \multicolumn{7}{|c|}{ Plants } \\
\hline Variable & \multicolumn{1}{|c|}{$\#$} & \% & Variable & \multicolumn{1}{c|}{$\#$} & $\%$ \\
\hline MEAN NDVI & 1053 & 65.8 & MEAN TCB & 1092 & 68.2 \\
\hline MEAN TCB & 892 & 55.7 & MEAN TCG & 668 & 41.7 \\
\hline CTW CV & 830 & 51.8 & MEAN NDVI & 636 & 39.7 \\
\hline MEAN TCG & 758 & 47.3 & MEAN TCW & 613 & 38.3 \\
\hline NDVI CV & 514 & 32.1 & NDVI CV & 482 & 30.1 \\
\hline MEAN CTW & 461 & 28.8 & TCW CV & 480 & 30.1 \\
\hline TCB CV & 292 & 18.2 & TCG CV & 449 & 28.0 \\
\hline TCG CV & 0 & 0 & TCB CV & 380 & 23.7 \\
\hline
\end{tabular}

5.5.3.3. Stepwise multiple regression models

Stepwise multiple regression (SWR) results using all eight explanatory variables demonstrated that long-term inter-annual dynamics in vegetation productivity, landscape brightness and moisture content are related to farmland biodiversity (Figure 5.8). The taxa groups responded differently to the predictors at $1 \times 1 \mathrm{~km}$ and $3 \times 3 \mathrm{~km}$ landscape scale and, unlike the results of the SWR models for MODIS data (Chapter 4.6.2.1), no consistent pattern of increase in $r$ from 1 $\times 1 \mathrm{~km}$ models to $3 \times 3 \mathrm{~km}$ models was observed. In fact, for most of the bird and butterfly models, $1 \times 1 \mathrm{~km}$ models were relatively stronger than $3 \times 3 \mathrm{~km}$ models. Each $1 \times 1 \mathrm{~km}$ landscape contained about 1,100 pixels while each $3 \times 3 \mathrm{~km}$ sample landscape contained about 10,000 pixels. The results demonstrate that at Landsat spatial resolution, model performance is not a function of sample site extent and a sample size of 1100 pixels is sufficient in portraying the 
relationship of biodiversity with landscape metrics. The outputs for SWR models of all years are presented in Appendix V.

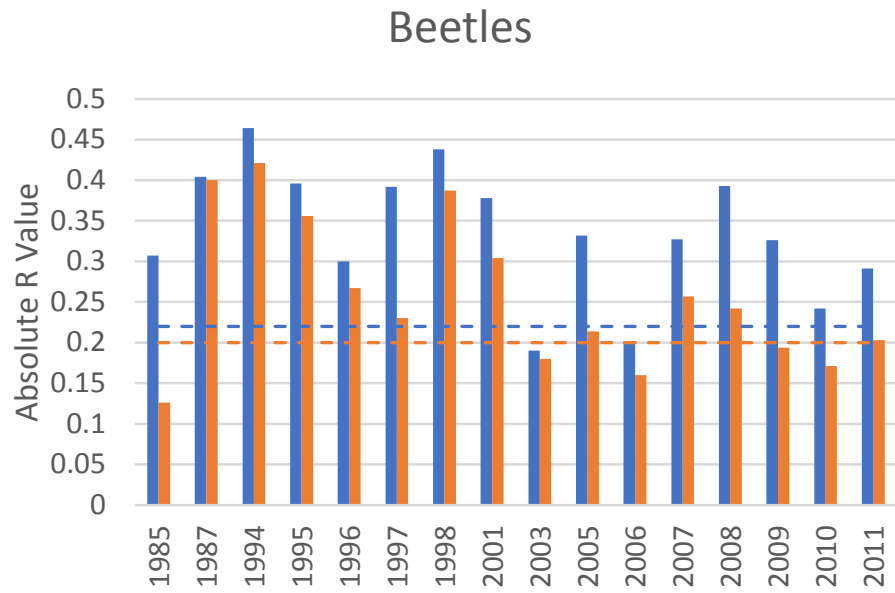

Butterflies

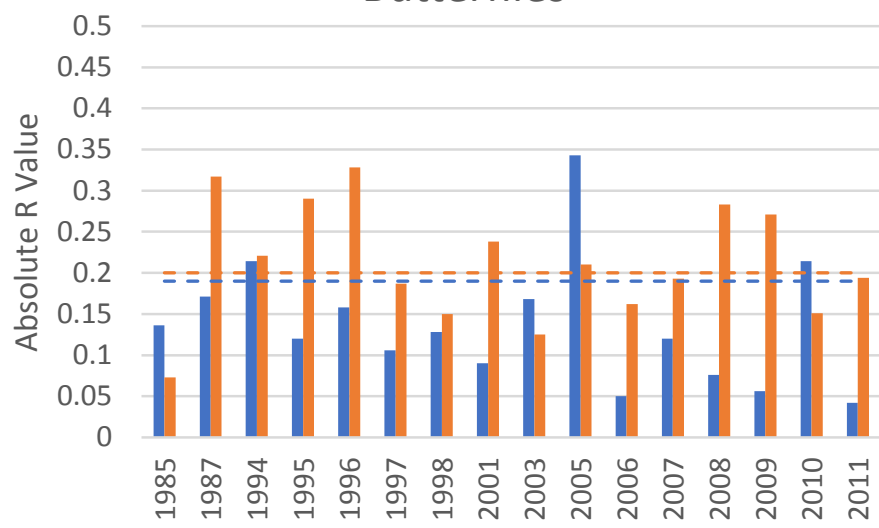

\section{Plants}

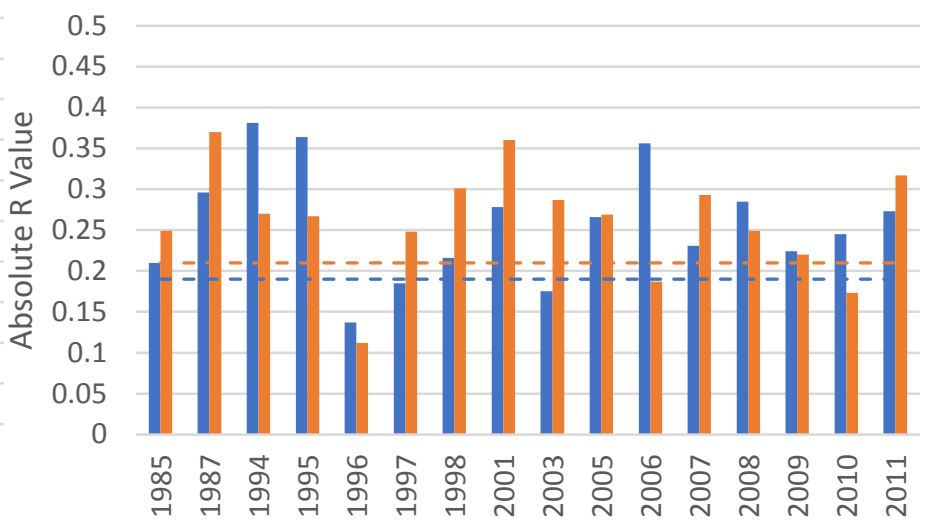

Birds

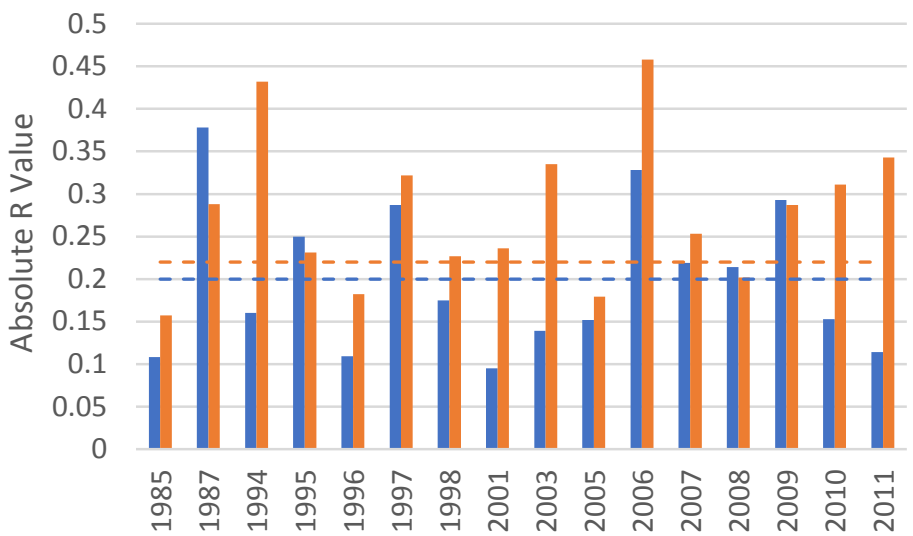

Figure 5.7. Absolute $r$ value for all models; Orange represents $1 \times 1 \mathrm{~km}$ models and blue represents $3 \times 3 \mathrm{~km}$ models. Dashed lines represent significant $r$ when $p=0.05$ for each corresponding landscape extent. Solid lines represent noticeable but non-significant trends of change in the strength of models from 1985 to 2011.

\subsubsection{Significant variables in SWR models and the magnitude and direction of effects}

The magnitude of the correlation between each entered explanatory variable and gamma diversity of a given taxon varies among years but generally shows the same direction. Table 5.9 gives the explanatory variables and the number of times each variable entered the SWR model for each taxon. The magnitude and direction of each variable is presented below for each taxon independently. Figures 5.8 to 5.11 present r-values with $95 \%$ confidence intervals. 
Table 5.9. The number of years out of 16 for which each remote sensing variable entered the SWR models for each taxon.

\begin{tabular}{|c|c|c|c|c|c|c|c|c|}
\hline & \multicolumn{2}{|c|}{ Birds } & \multicolumn{2}{c|}{ Beetles } & \multicolumn{2}{c|}{ Butterflies } & \multicolumn{2}{c|}{ Plants } \\
\hline & $1 \times 1$ & $3 \times 3$ & $1 \times 1$ & $3 \times 3$ & $1 \times 1$ & $3 \times 3$ & $1 \times 1$ & $3 \times 3$ \\
\hline Mean NDVI & 1 & 0 & 4 & 1 & 1 & 0 & 1 & 0 \\
\hline NDVI CV & 1 & 2 & 1 & 4 & 2 & 0 & 2 & 1 \\
\hline Mean TCB & 3 & 2 & 1 & 2 & 0 & 0 & 7 & 8 \\
\hline TCB CV & 2 & 2 & 0 & 3 & 0 & 0 & 1 & 1 \\
\hline Mean TCG & 2 & 2 & 2 & 2 & 1 & 0 & 2 & 2 \\
\hline TCG CV & 4 & 2 & 3 & 3 & 2 & 0 & 1 & 1 \\
\hline Mean TCW & 6 & 1 & 5 & 3 & 2 & 0 & 1 & 2 \\
\hline TCW CV & 3 & 2 & 1 & 4 & 3 & 2 & 3 & 3 \\
\hline
\end{tabular}

\subsection{Beetles}

Among the landscape predictors for $1 \times 1 \mathrm{~km}$ beetle models, all variables except TCB CV entered models at least in one year. The most frequently entered variable was Mean TCW which entered the models in five of the 16 years, $(1994,1996,1998,2001$ and 2007). It was the first variable entered in 1994 and the only variable entered in 1996 and 2007 models. Other variables that entered models in three or more years were: Mean NDVI in four years (the only variable entered 1997, 2005, 2008 and 2011) and TCG CV in three years (the first variable entered 1987, 1995 and 2011). Six of 16 models were single variable models (1996, 1997, 2005, 2007, 2008 and 2011), while four models had two variables (1987, 1994, 1998 and 2001) and 1995 was the only three variable model. No variables were significant for 1985, 2003, 2006, 2009 and 2010.

All variables entered $3 \times 3 \mathrm{~km}$ models at least in one year. The most frequently entered variables were TCW CV and NDVI CV (both entered in 4 years). TCW CV was the first variable entered in 1997, 2001, 2008 and 2009 models, while NDVI CV was the only variable entered for 2005, the first variable entered for 1998, and the second variable entered for 2001 and 2008. Other variables that entered models in three or more years were: TCB CV three years (first variable entered for 1994, 1995 and only variable in 2007), TCG CV three years (1985, 1987 and 1997), and Mean TCW three years (1996, 2009 and 2010). Eight of 16 models had one variable (1985, 
1987, 1996, 2005, 2006, 2007, 2010 and 2011) and seven had two variables $(1994,1995,1997$, 1998, 2001, 2008 and 2009). No variables were significant for 2003.

Figure 5.9 demonstrates the direction and magnitude of the r-values with $95 \%$ confidence intervals. Significant variables in $1 \times 1 \mathrm{~km}$ and $3 \times 3 \mathrm{~km}$ models generally demonstrated a negative relationship with beetle gamma diversity, except Mean NDVI which was positive. In addition, Mean TCW in 2001 and TCG CV in 1995 in $1 \times 1 \mathrm{~km}$ models demonstrated a significant positive relationship with gamma diversity which contrasted with the other years for both $1 \times 1 \mathrm{~km}$ and 3 $\times 3 \mathrm{~km}$ extents.

The results of SWR models are in agreement with those of RFR for $1 \times 1 \mathrm{~km}$ models since Mean TCW and Mean NDVI were the most frequently entered variables and were deemed to be amongst the most important variables in RFR. For $3 \times 3 \mathrm{~km}$ models however, the variables that frequently entered SWR models were not amongst the top variables of RFR.

The positive relationship of Mean NDVI and the negative relationship of Mean TCB and beetle gamma diversity indicated that beetles prefer landscapes with more vegetation cover and a lower amount of bare soil. However, three models did show a weaker but still significant negative relationship with Mean TCG. A mix of significant positive and negative relationships for Mean TCW may be indicative of the difference in habitat wetness preference of beetle species. Some beetle species that were surveyed (e.g., Genus Cicindela, Amara, Bembidion, Chlaenius and Agonum) were species that prefer moist areas, wet sand and mud in open habitats near ponds and lakes, while others (e.g., Genus Harpalus and Diplocheila) prefer drier habitats (Arnett and Thomas, 2000; Pearson et al., 2015). For the spatial variability variables, NDVI CV, TCG CV and TCW CV demonstrated significant negative relationships in all or most models, which 
indicated that lower beetle diversity in 2011-12 occurred in landscapes that had higher spatial variability in vegetation amount and moisture in almost all years from 1985 to 2011.

a Beetles $1 \times 1 \mathrm{~km}$

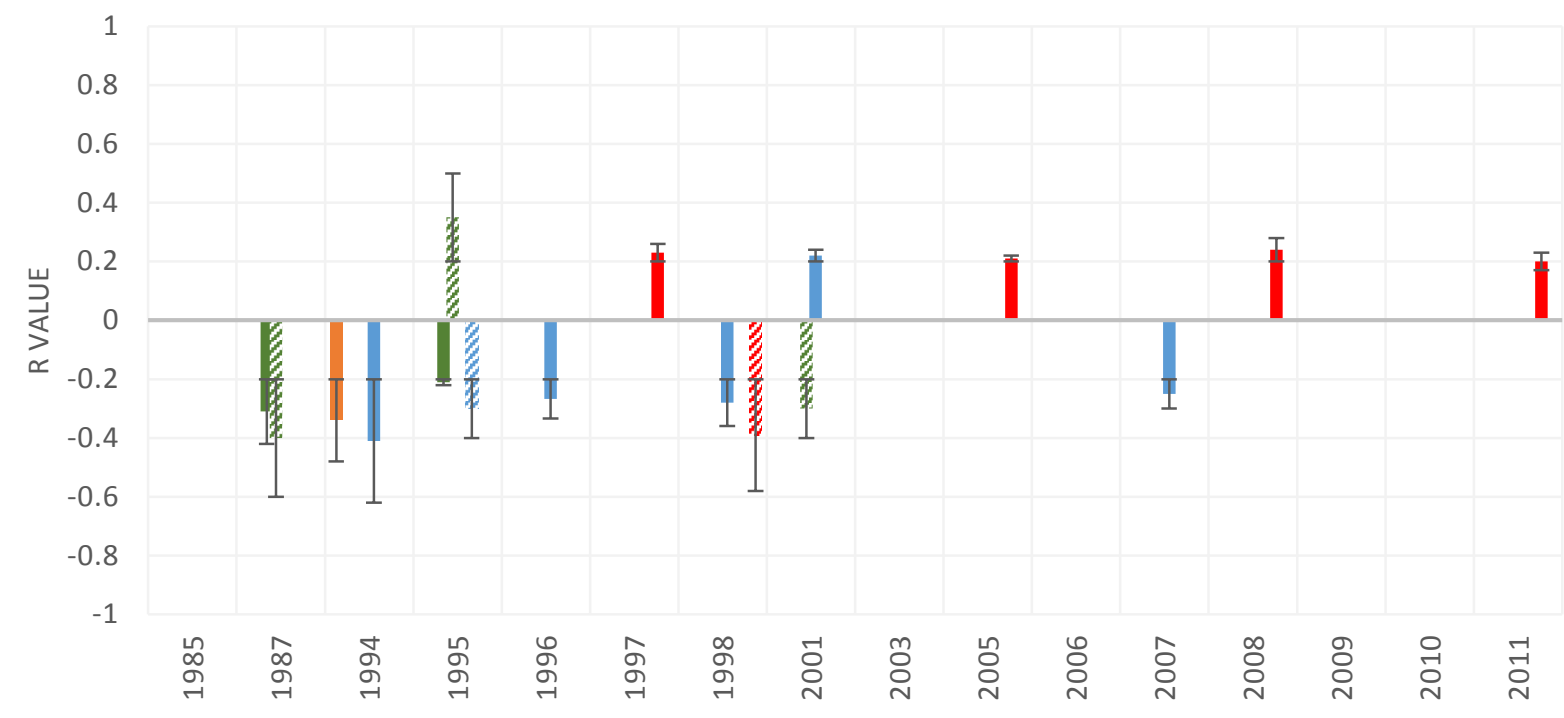

$\square$ Mean TCB $\approx$ TCB CV $\square$ Mean TCG $\approx$ TCG CV $\square$ Mean TCW $\approx$ TCW CV MEAN_NDVI $\boldsymbol{z}$ NDVICV

$b$

Beetles $3 \times 3 \mathrm{~km}$

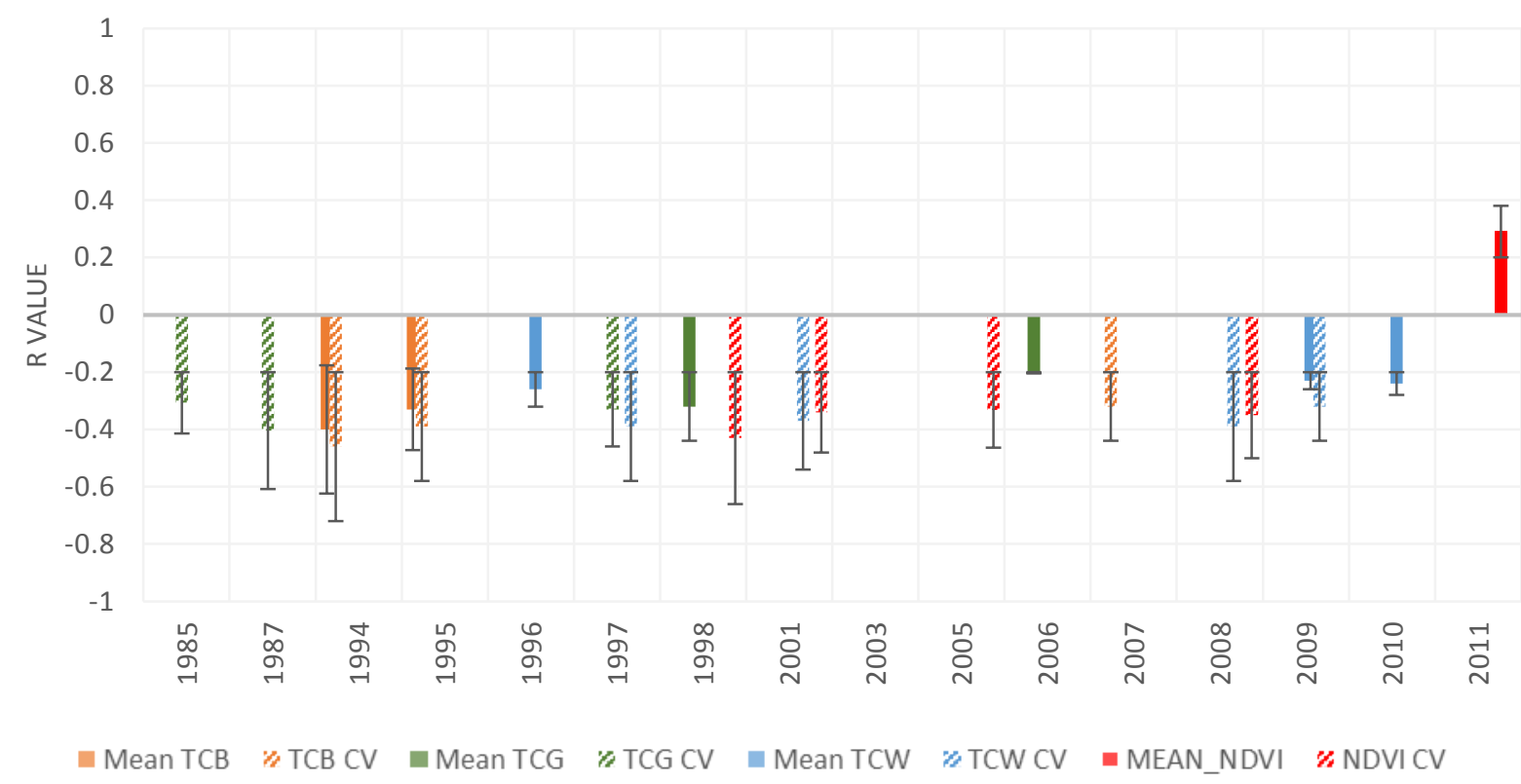

Figure 5.8. R-values and their $95 \%$ confidence intervals for beetle gamma diversity in 1 $\times 1 \mathrm{~km}$ (a) and $3 \times 3 \mathrm{~km}$ (b) models. The explanatory variables shown are those that produced significant models in one or more years. 


\subsection{Plants}

Figure 5.10 shows the direction and magnitude of variables entered into plant models for each year and extent. The variables that entered $1 \times 1 \mathrm{~km}$ and $3 \times 3 \mathrm{~km}$ plant models demonstrated a general agreement. Mean TCB was the most frequently entered variable for both extents as it entered $1 \times 1 \mathrm{~km}$ models in 7 years $(1985,1987,1994,2001,2003$ and 2011) and $3 \times 3 \mathrm{~km}$ models in 8 years $(1985,1994,1995,2001,2006,2007,2008$ and 2011). It was the only variable entered in four $1 \times 1 \mathrm{~km}$ and five $3 \times 3 \mathrm{~km}$ models, although not the first variable entered the other models. It demonstrated significant negative relationships, indicating that landscapes with higher amounts of exposed soil (or other non-vegetated surfaces such as buildings) contained lower diversity noncrop plants. This was in agreement with the results of RFR as Mean TCB was the first and second most important variable in $3 \times 3 \mathrm{~km}$ and $1 \times 1 \mathrm{~km}$ models, respectively.

TCW CV entered $1 \times 1 \mathrm{~km}$ and $3 \times 3 \mathrm{~km}$ models in 3 models each $(1 \times 1 \mathrm{~km}$ in 1997 , 2007 and 2008, and $3 \times 3 \mathrm{~km}$ in 1994, 1997 and 2010). The relationship was negative for $1 \times 1$ $\mathrm{km}$ models, but a mix of positive and negative for $3 \times 3 \mathrm{~km}$ (positive for 1994 and 2010; negative for 1997). This demonstrated that the effect of spatial variability in landscape moisture on noncrop plants was variable throughout years and landscape extents. Other variables did not enter more than two of the 16 plant models.

In $1 \times 1 \mathrm{~km}$ models, 9 of 16 models were single variable models, while two variables entered 1998, 2003 and 2007 models, and three variables entered the 2003 model. No variables were entered for 1996, 2006 and 2010. In $3 \times 3 \mathrm{~km}$ models 10 of 16 models were single variable (1985, 1987, 1997, 1998, 2001, 2005, 2007, 2008, 2009, 2010 and 2011), 1994 and 1995 were two variable models, three variables entered the 2006 model, and no variables were entered for 1996 and 2003. 


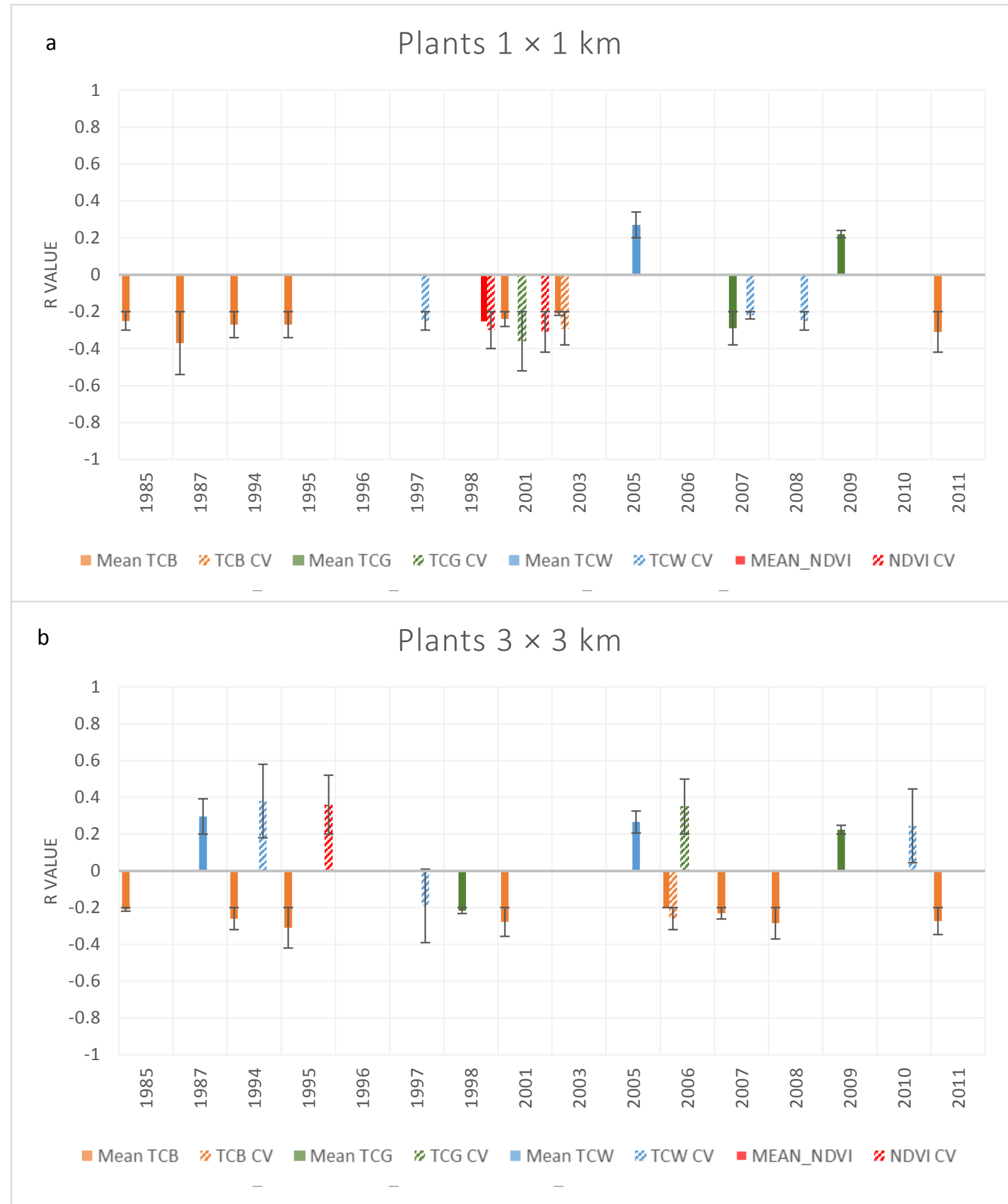

Figure 5.9. R-values and their $95 \%$ confidence intervals for plant gamma diversity at $1 \times 1 \mathrm{~km}$ (a) and $3 \times 3 \mathrm{~km}$ (b) models. The explanatory variables shown are those that entered SWR regression and produced significant models in one or more years. 


\subsection{Birds}

Generally, birds demonstrated a stronger relationship with explanatory variables of $1 \times 1 \mathrm{~km}$ extent compared to $3 \times 3 \mathrm{~km}$. Figure 5.11 demonstrates the magnitude and direction of bird models. Overall, no variables were entered into models for more than half the years. Mean TCW entered six $1 \times 1 \mathrm{~km}$ models (1994, 1998, 2003, 2006, 2007 and 2011), with a negative relationship in all of these except 2007. Other variables that entered $1 \times 1 \mathrm{~km}$ models three or more times were: Mean TCB (1994, 2006 and 2010) and TCG CV (1994, 2001, 2006 and as the first variable in 2010). Seven of 16 models were single variable models, while 3 models had two variables $(2009,2010,2011)$.

For 1994, four variables and for 2006 five variables entered the model. Birds at $1 \times 1 \mathrm{~km}$ are the only taxon that created models with more than three variables. No significant variables were entered for 1985, 1996, 2005 and 2008. At $3 \times 3 \mathrm{~km}$ no variables were entered into more than two models and 9 of 16 regressions did not produce a significant model.

In terms of the most significant variables, these results do not agree with the RFR results. In RFR Mean and CV of NDVI were the most important variables for both landscapes extents, while Mean NDVI entered only one $1 \times 1 \mathrm{~km}$ model and NDVI CV entered one $1 \times 1 \mathrm{~km}$ and two $3 \times 3 \mathrm{~km}$ models. Mean TCW was the most consistently entered variable, in $1 \times 1 \mathrm{~km}$ models and almost always negative, indicating that bird diversity was lower in wetter landscapes. For example, in 1994 and 2006 where Mean TCW demonstrated the strongest relationship with bird diversity, most of the wetter landscapes (such as H_H_2, L_H_86, H_L_56 and L_L_110) contained less than 10 bird species. This could be due to the habitat preferences of birds, since some of the surveyed birds prefer woody areas, fields or open areas, and forest edges (e.g., Spinus tristis, Bombycilla cedrorum, Eremophila alpestris) while others prefer wetter areas and areas near water (e.g., Melospiza georgiana, Tachycineta bicolor, Bartramia longicauda) (Holloway, 
2003). Other variables such as NDVI CV, TCG CV and Mean TCB each entered in less than four models and they were not deemed to be strong indicators of bird diversity of 2011 and 2012 . The negative relation of NDVI CV with bird diversity was in agreement with the results of Chapter 4 and that of Duro et al. (2014) as mentioned in Chapter 4.5.2.3. 


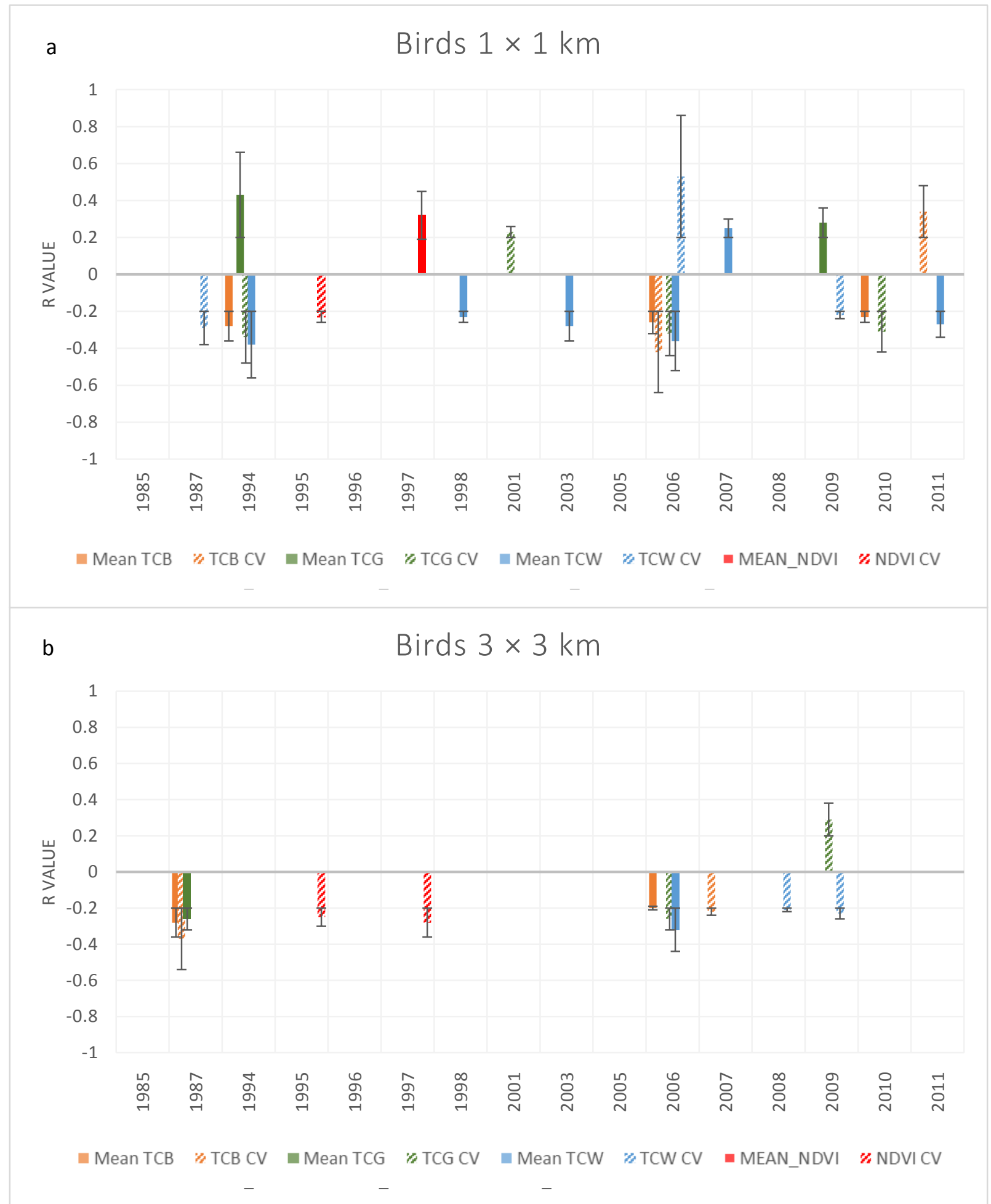

Figure 5.10. R-values and their 95\% confidence intervals for bird gamma diversity at $1 \times 1 \mathrm{~km}$ (a) and $3 \times 3 \mathrm{~km}$ (b) models. The explanatory variables shown are those that entered SWR regression and produced significant models in one or more years. 


\subsection{Butterflies}

Figure 5.12 demonstrated that fewer significant models were produced for butterflies, and only two for $3 \times 3 \mathrm{~km}$. In $1 \times 1 \mathrm{~km}$ models, TCW CV was entered for three years (only variable in 1987 and the first variable entered 2008 and 2009). All other variables were entered into two or fewer models. All entered variables demonstrated negative relationships with butterfly gamma, except Mean TCG.

The strength of the significant models was also generally weaker than those for the other taxa. Models for 1987, 1994, 2001 and 2005 were single variable models, while two variables were entered into 1996, 2008 and 2009 models. No variables were entered for eight years (1985, 1997, 1998, 2003, 2006, 2007, 2010 and 2011). For the $3 \times 3 \mathrm{~km}$ extent, TCW CV was the only predictor that entered models and only in 1994 and 2010. No variable entered other years. These results were not in agreement with those of RFR, since the entered variables in SWR models did not occur frequently in RFR.

TCW CV was the most significant variable for both $1 \times 1 \mathrm{~km}$ and $3 \times 3 \mathrm{~km}$ butterfly models, but with different directions. Given its low number of occurrences in models (3 and 2, for $1 \mathrm{~km}$ and $3 \mathrm{~km}$ extents, respectively), little can be conclusively stated, other than the possibility that some of the surveyed species prefer more moist habitats and adjacency to bogs (e.g., Phyciodes cocyta, Phyciodes tharos, Cercyonis pegala, Lycaena Hyllus), while others prefer wooded areas (e.g., Genus Vanessa and Genus Limenitis). 


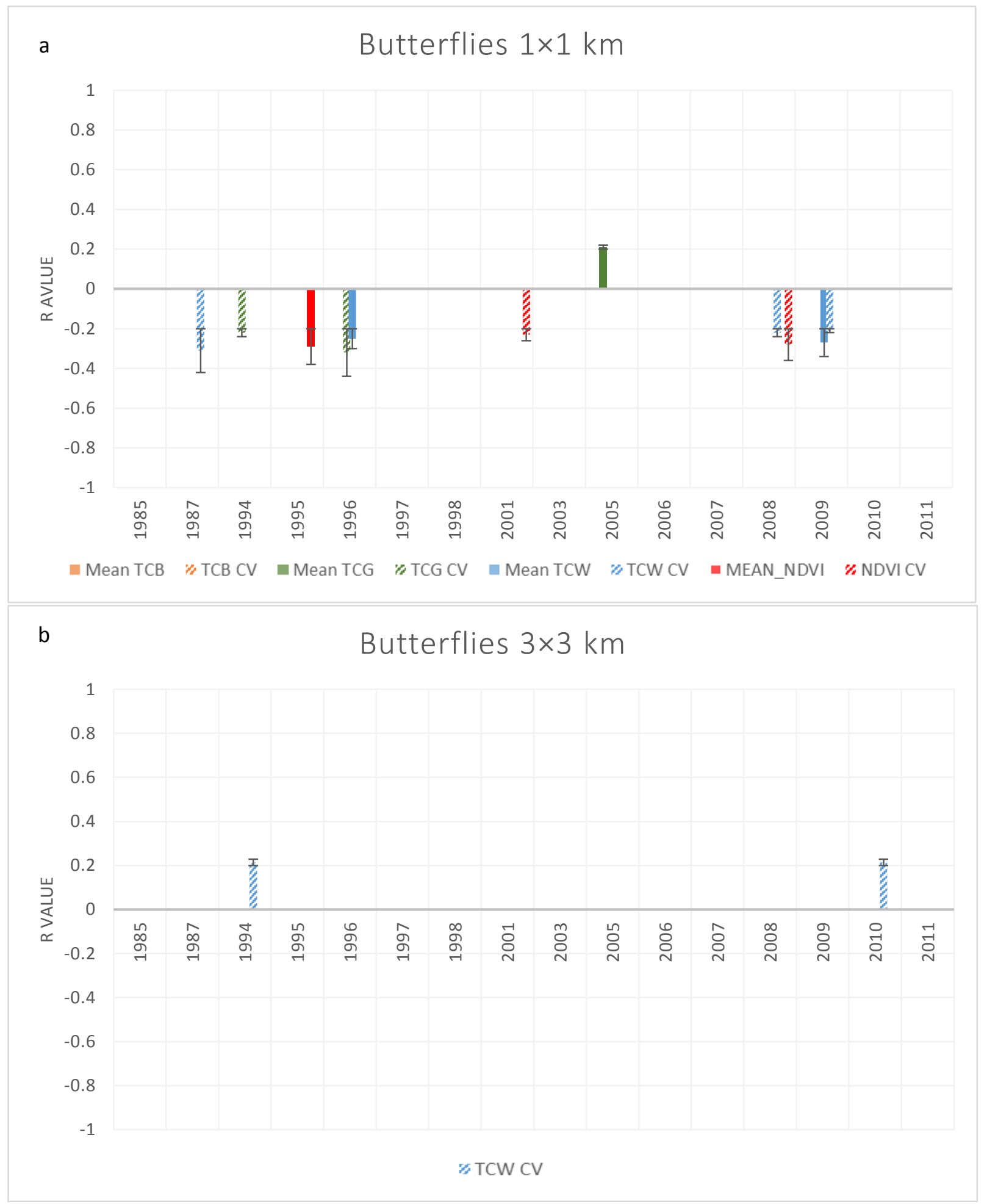

Figure 5.11. R values and their $95 \%$ confidence intervals for butterfly gamma diversity at $1 \times 1$ $\mathrm{km}$ (a) and $3 \times 3 \mathrm{~km}$ (b) models. The explanatory variables shown are those that entered SWR regression and produced significant models in one or more years. 


\subsubsection{Model residuals}

Models residuals were normally distributed. Residual plots demonstrate a scattered distribution along the $\mathrm{x}$-axis (the explanatory variable), suggesting a linear and homoscedastic relationship between the predictors and gamma diversity. The Breusch-Pagan heteroscedasticity test (Breusch and Pagan 1979) was run on the model residuals using a significance level of $p=0.05$. The $p$ value range from 0.45 to 0.1 suggested non-significant and homoscedastic relationships. As an example, the residual plot of Mean TCB against beetle gamma diversity in 2011 is presented in Figure 5.13.

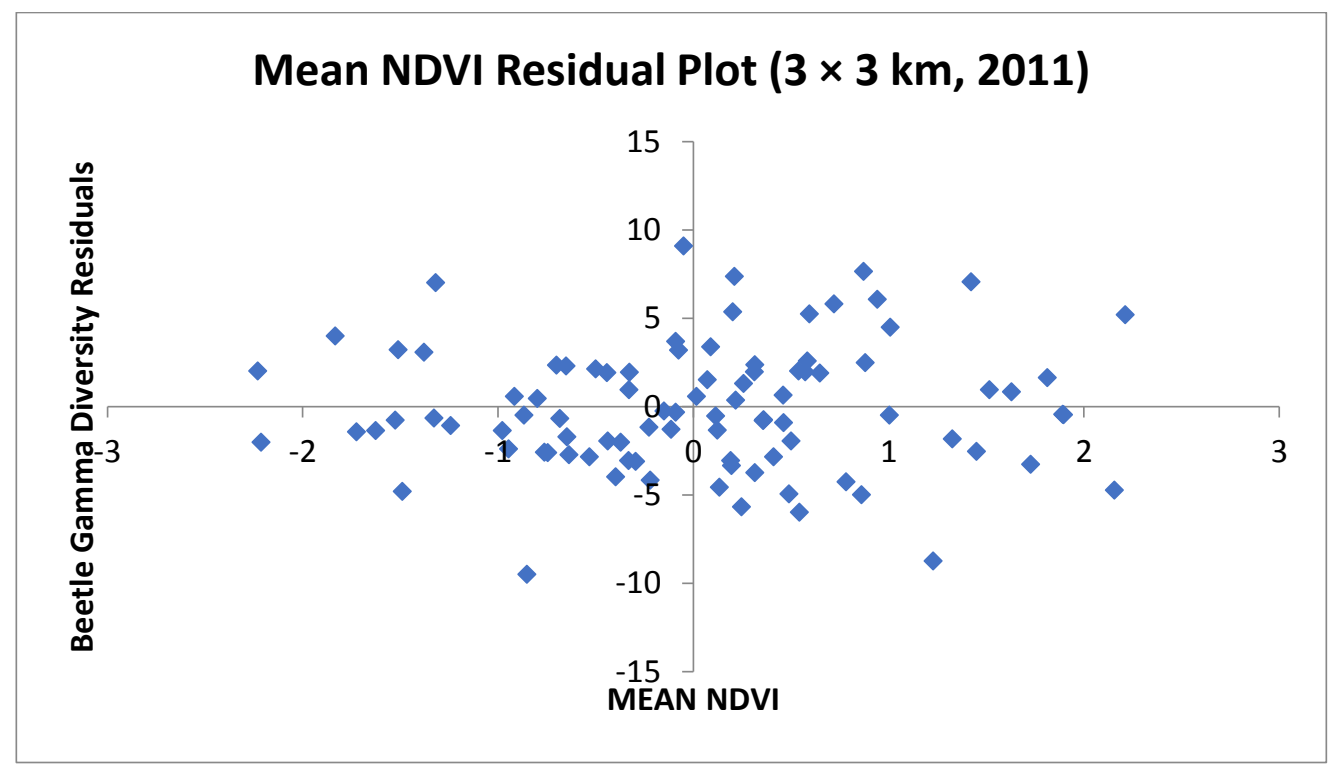

Figure 5.12. Example residual plot for the $3 \times 3 \mathrm{~km}$ beetle model of 2011 with Mean NDVI as the entered explanatory variable.

\subsubsection{Evaluating potential lag effect}

The analysis of potential lag effect was conducted on $1 \times 1 \mathrm{~km}$ sample landscapes. For beetles, birds and butterflies, Mean TCW and for plants Mean TCB were selected as the best variables which entered individual models most frequently in SWR. Lag effect was tested for each species individually by creating models as described in Section 5.5.3. Beetles, birds and butterflies did not show any patterns of lag effect as adding the variables of past years to the model caused model 
inflation and a decrease in the Pearson r. For plants, on the other hands, the r-value was low for M1 to M10 but demonstrated a jump in M11, where the Mean TCB of 1997 were added (Figure 5.13). This suggested a stronger relation between Mean TCB of 1997 and gamma diversity of plants in 2011-2012. Different scenarios were tested to verify this relation. For example, TCB of 1997 and 2011 were modelled individually against plant gamma diversity to test if removal of other years from the model would create a stronger relationship but an r-value of 0.25 , equal to M1 and lower than M11, was obtained. This showed that removal of other years did not lead to a stronger model.

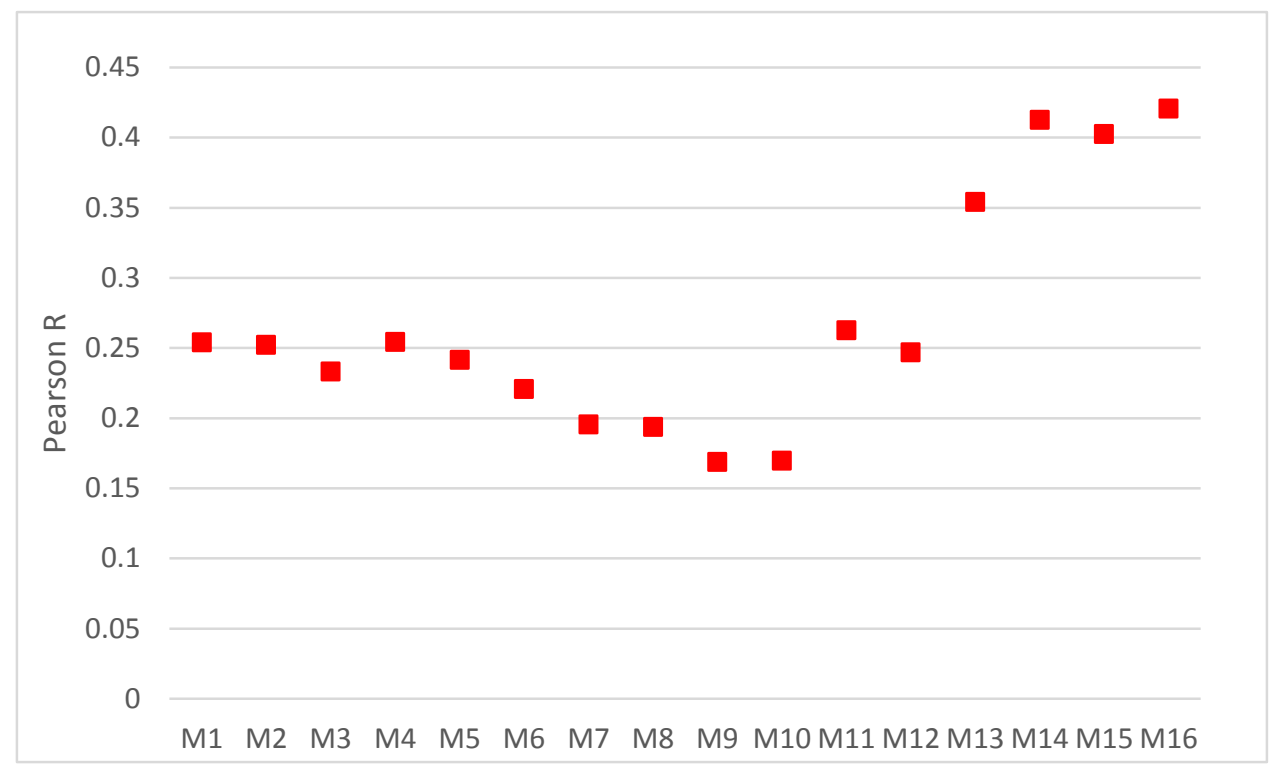

Figure 5. 13. Pearson $r$ for lag effect models of Mean TCB against plant gamma diversity. A jump in the r value was evident in M11 where the Mean TCB of 1997 was added to the model.

Investigating climate factors revealed a $20 \%$ lower than average total July precipitation in 1997 (Table 6.1.) which may be associated with higher landscape brightness and hence lower non-crop plant diversity. However, lower total July precipitation was reported in the following years while the same effect on the model performance was not noted for those years. 
To test the effect of landscape change on the models, a similar method as in Section 4.5.2.7 was used by removing Mean TCB of 1997 from the models. The r-value remained high in models after its removal, which demonstrated that the landscape condition of 1997 was reflected in the previous years. This may be due to the potential landscape changes including the removal of natural patches which had led to higher landscape brightness in the years following 1997.

\subsection{Discussion}

\subsubsection{Interpretation of main findings and relations to previous work}

The first objective of this study was to evaluate the relationships between mid-summer Landsatderived continuous metrics, including vegetation indices, TCT components and their spatial variability, with gamma diversity of farmland taxa in a mixed agricultural landscape in eastern Ontario. The RFR and SWR models were weak overall, however, they suggested trends of the spatial mean and variability of NDVI in relation to the diversity of birds, butterflies, beetles and wild plants to varying degrees. This result is in agreement with Sheeren et al. (2014), St. Louis et al. (2014) and Duro et al. (2014), who found these variables to be significant when modelling biodiversity in different landscapes. In addition, the significant results for TCT components, particularly TCW (all taxa) and TCB (plants) concurs with Warren et al. (2014) who showed the significance of a similar band transformation technique (PCA), especially the first principal component (as equivalent of $\mathrm{TCB}$ ) in relation to plant species richness (alpha diversity). In addition, the spatial variability of NDVI and TCT variables demonstrated significant relationships with gamma diversity, which is in agreement with Duro et al. (2014) who found significant relationships between bird diversity and spatial variability in landscape productivity and variability in the local spatial clustering of productivity.

The second objective of this study was to identify the most significant variables in RFR and SWR models of biodiversity. Overall, 12800 RFR models were produced for the four selected 
taxa (1600 models for each taxon at each landscape extent) and Mean NDVI over a landscape, as a measure of average vegetation biomass and productivity, was selected by RFR as the most significant landscape variable in biodiversity models (21\% of all models). In addition, 128 SWR regression models were produced (16 models for each taxon at each landscape extent) 82 (64.1\%) of which were significant ( 26 beetles, 19 birds, 10 butterflies, 27 plants). Although the average of vegetation productivity, as represented by vegetation indices such as NDVI, has been commonly found to be associated with biodiversity (as reviewed by Nagendra, 2001 and Gillespie et al., 2008), Mean NDVI did not frequently enter the subsequent SWR models, and the most significant variables were Mean TCB (entered in 23 models or 17.9\%) and TCW CV (entered in 21 models or $16.4 \%)$.

Mean TCB as a measure of the amount of radiance detected from bare soil and humanmade features in a landscape was selected by RFR and SWR models of plants, beetles and birds as a significant landscape variable in biodiversity models. It demonstrated a negative relationship with gamma diversity of these taxa for all years in which it was entered into a model, indicating that higher amounts of uncultivated bare soil, asphalt and roads, as well as other human-made features, may have had a negative effect on diversity. This result is in agreement with many ecological studies which associated roads and other human-made features with lower biodiversity (e.g. Findlay and Bourdages, 2000; Forman and Deblinger, 2001; Karlson et al., 2014; Bennett, 2017).

TCW CV, as representative of spatial variability in landscape moisture content, did not appear amongst the most frequent variables in RFR but was the second most frequent variable entered in SWR models. However, relationship direction was inconsistent over all taxa, years, and landscape extents, being negative for 16 of 21 models (76.2\%) and positive for 5 models $(23.8 \%$ - two butterfly and two plant models at $3 \times 3 \mathrm{~km}$, and one bird model at $1 \times 1 \mathrm{~km})$. Given 
various habitat preferences of species (examples of wetland and dryland beetles, birds and butterflies presented in 5.6.2.5.1, 5.6.2.5.3 and 5.6.2.5.4, respectively), a positive relationship of TCW CV with gamma diversity would be expected, as more diverse landscapes are able to provide the habitat requirements of a larger variety of dryland to wetland species, while a negative relationship was evident for the majority of models. This inconsistency could be due to other biogeophysical factors and climate factors such as year-to-year average annual temperature and total precipitation variations.

The third objective of this study was to identify the taxon (taxa) which demonstrated the strongest relationships with the explanatory variables. As mentioned, the SWR models were generally weak, but plants and beetles created more significant models compared to birds and butterflies; $84.4 \%$ of all plant and $81.2 \%$ of all beetle models were of statistical significance. For plants at $1 \times 1 \mathrm{~km}$ and $3 \times 3 \mathrm{~km}$ extents only 3 and 2 models were not significant, respectively. Mean TCB was the most significant variable as it was entered into seven $(43.75 \%)$ and eight $(50 \%)$ models for $1 \times 1 \mathrm{~km}$ and $3 \times 3 \mathrm{~km}$, respectively, and demonstrated a constant negative relationship with gamma diversity for both taxa. In 2011 models (i.e., the year closest in time to the biodiversity assessments), Mean TCB was the only variable which entered both $1 \times 1 \mathrm{~km}$ and $3 \times 3 \mathrm{~km}$ models, reinforcing its importance as a predictor of plant diversity. For beetles at $1 \times 1$ $\mathrm{km}$ and $3 \times 3 \mathrm{~km}$ extents only 5 and 1 models out of 16 were not significant, respectively. Mean TCW and TCW CV were the most significant variables for beetle models $(37.5 \%$ of $1 \times 1 \mathrm{~km}$ and $43.75 \%$ of $3 \times 3 \mathrm{~km}$ models) and almost always with a negative effect. Mean NDVI was positively related to beetle diversity in all five models in which it was entered, and in each case including 2011 at both $1 \times 1 \mathrm{~km}$ and $3 \times 3 \mathrm{~km}$ extents, it was the only variable entered. The number of significant models was much lower for butterflies and birds (31.25\% and 59.37\%, respectively), 
especially at the $3 \times 3 \mathrm{~km}$ extent, which may indicate that a larger sample extent was not appropriate for portraying the relationships of these taxa to the given Landsat-based metrics.

The fourth objective of this study was to compare the relative effect of sample landscape extent on model performance. The results showed that unlike MODIS models, no clear pattern of increasing model performance from $1 \times 1 \mathrm{~km}$ to $3 \times 3 \mathrm{~km}$ models could be observed. While the model significance improved from $1 \times 1 \mathrm{~km}$ to $3 \times 3 \mathrm{~km}$ for beetles, for birds and butterflies most of $1 \times 1 \mathrm{~km}$ models were more significant than $3 \times 3 \mathrm{~km}$ models. The model performance in plants was not found to be a function of sample landscape size as model significance varied for $1 \times 1$ $\mathrm{km}$ and $3 \times 3 \mathrm{~km}$ in different years. Many previous biodiversity modeling studies had been conducted at multiple spatial scales and various responses of species to different spatial scales was captured (e.g. Mooreno and Halffter, 2001; Cunningham et al., 2014; Guyot et al., 2017). These results emphasized the importance of the land cover types, ecosystem and other environmental variables, species specific factors and the spatial and spectral properties of remotely sensed data in the response of species to multiple spatial scales.

The fifth objective of this study was to compare the response of 2011-12 biodiversity to past (1985-2010) and concurrent (2011) Landsat-derived explanatory variables to determine if a lagged response was evident. Of all 2011 models, beetle models for both extents demonstrated significant positive relationships with Mean NDVI, while plant models for both extents demonstrated significant negative relationships with Mean TCB. Butterflies did not show a significant relationship in 2011 and birds only showed significant relationship at $1 \times 1 \mathrm{~km}$ (positive relationship with TCB CV and negative relationship with Mean TCW). However, similar to the MODIS models as explained in Chapter 4.7.1, the strength of the relationships for 2011 was not consistently stronger or weaker than for past years, indicating that in case of absence of same-year appropriate reflectance data, data from the past could be utilized. 
Conversely, since the strength of relationships using pre-2011 predictor data was generally not significantly stronger than for 2011 models, there was no evidence of a time lagged response of birds, butterflies and beetles to the explanatory variables. However, plants demonstrated a time lagged response to Mean TCB as the model strength improved by adding the variable of 1997 to the model. This may be due to temporal landscape changes or changes in the crop types in the study region. The results of the analysis of time lagged response of species to landscape variables are in agreement with those of Krauss et al. (2010), Dullinger et al. (2012) and Findlay and Bourdages (2000). Krauss et al. (2010) found a strong relationship between the past habitat area and current wild plant richness in semi-natural grasslands, while butterflies were related to the current landscape area as opposed to the past one. Dullinger et al. (2012) found a time lagged response of the native plants to habitat size in the European Alps. Findlay and Bourdages (2000) captured a lag effect in the response of birds and wild plants to road construction in wetlands. It should be noted that these studies were not conducted in agricultural ecosystems and the landscape variables that were compared to biodiversity were habitat loss and fragmentation and habitat area as opposed to the average and variability of landscape productivity, brightness and moisture content.

This study highlighted the potential of long-term time series of Landsat to derive continuous variables and their relation with biodiversity. Landsat had been used previously as an effective dataset for biodiversity modeling. For example, in a study that preceded this research using the same field biodiversity data, Duro et al. (2014) emphasized the capability of Landsatderived continuous variables to create strong biodiversity models. In addition, St. Louis et al. (2014) highlighted the suitability of Landsat-derived continuous variables to model avian diversity in a semi-arid environment. However, these studies lack a temporal dimension since they modeled biodiversity only at one point in time. The presented work attempted to address this 
gap by using the Landsat long-term temporal archive to model the species response to remote sensing variables in three decades.

Comparing model fit of RFR and SWR models of Landsat with those of MODIS (presented in Chapter 4) demonstrated that despite better Landsat spatial resolution and additional spectral information (e.g. SWIR), the MODIS variables, including phenological variables generally produced better models.

\subsubsection{Limitations and recommendations for future research}

One of the main limitations of this work was low temporal variability of Landsat data and unavailability of cloud free mid-summer images (July-August 15). This issue caused gaps in the time series for years in which cloud free images were not available. In addition, some of the images were acquired on dates outside the mid-summer range by a few days. Being at different growth stages might have caused systematic inconsistency in some of the remote sensing variables, especially measures of vegetation biomass and productivity.

Another limitation of this work as explained in Chapter 4.6.2 was the temporal limitation of biodiversity data. The same recommendations are suggested to overcome the limitations of modeling biodiversity of one year against landscape variables of individual past years.

\subsection{Conclusions}

This study attempted to address a gap in the literature in evaluating the response of biodiversity to spatial and temporal changes in habitat by using long-term time series of Landsat 5 TM from 1985 to 2011. Measures of vegetation productivity, vegetation and soil moisture and overall landscape brightness were modeled against farmland biodiversity surveyed in 2011 and 2012 . The average and spatial variability of TCB, TCG and TCW were used for the first time as explanatory variables in biodiversity models and TCB and TCW were found to be related to 
biodiversity. No significant difference in the relationship of past and present explanatory variables derived from individual years with present biodiversity was found, although plants demonstrated a stronger relationship with TCB of past years compared to 2011. 


\section{Chapter 6: MODIS and Landsat inter-annual landscape trends and their relationships with farmland biodiversity}

\subsection{Introduction}

Land use change, especially the transformation of natural and semi-natural lands to farmlands, is one of the major drivers of global biodiversity loss. Major land transformations to agriculture have largely occurred in the past few decades in Europe and North America (Poschlod et al., 2005; Reidsma et al., 2006). In Canada the amount of natural lands dropped from over 300 million hectares in the 1950s to 240 million hectares in the 2000s, while the amount of agricultural lands, many under intensive practices, increased from 60 million acres in the 1950s to 90 million acres in the 2000s (Statistics Canada, Census of Agriculture, 2006).

Agricultural intensification involves several types of processes including conversion of areas of natural vegetation (e.g., forests, wetlands) to agriculture, reduction or removal of natural vegetation at field margins to increase the cropped area of a field or to merge two fields into one, use of similar crop types between fields, and increased use of management techniques such pest and weed control, fertilization, irrigation and artificial drainage systems (Hanley et al., 2012). These result in changes in vegetation productivity across landscape. Natural lands such as forests, shrubland and grasslands are often associated with larger biomass, greater photosynthetic capacity and higher primary productivity, while agricultural landscapes are simplified lands with lower vegetation productivity.

Evaluating the impacts of land transformation and other processes of agricultural intensification on biodiversity requires long-term monitoring. These relationships have largely been studied in Europe and North America. For example, Reidsma et al. (2006) assessed longterm agricultural biodiversity in the European Union. They used measures of ecosystem quality (characterized as ecosystem disturbance ranging from 0 to $100 \%$ as an indicator of biodiversity, 
where $100 \%$ indicated an undisturbed natural situation, while $0 \%$ represented a completely transformed and destroyed ecosystem with no wild species left. They found the lowest ecosystem quality in intensively used agricultural lands, particularly in lowlands of the Netherlands and France. Senapathi et al. (2015) studied the impact of 80 years of land cover change on the bee and wasp communities in England and revealed that in $75 \%$ of their $1 \times 1 \mathrm{~km}$ landscapes, a significant decline in bee and wasp richness occurred and these declines were associated with arable land expansion within the landscapes. Jiang et al. (2003) studied the long-term impacts of land use on plant diversity in the Loess Plateau of China, detecting a positive relationship between land cover diversity and plant diversity, and emphasizing the role of forest and shrub land covers in supporting native plant species in the region. Herrando et al. (2014) studied the effects of the opposite process of agricultural land abandonment on avian communities in Catalonia between 2002 and 2011. The results showed that bird abundance significantly responded (positively or negatively) to vegetation encroachment associated with reduced livestock grazing and wood harvesting, and to farm abandonment, which shifts cultivated lands into natural habitats.

Inter-annual changes in primary productivity due to land transformation can be monitored and quantified using the long-term archive of multispectral remote sensing data. Many remote sensing based algorithms and indices have been developed to quantify vegetation productivity and biomass. The Normalized Difference Vegetation Index (NDVI, see section 2.3.1) is one of the most popular algorithms that is used as an indicator of green vegetation quantity and health and to quantify temporal changes in vegetation. High temporal resolution data such as from the Advanced Very High Resolution Radiometer (AVHRR) with a long temporal archive since 1978 have been used to detect long-term temporal changes in vegetation. A repeat time of 1-2 days enables creation of weekly composites to achieve consistent and precise yearly trends. For example, Slayback et al. (2003) and Tateishi and Ebata (2004) used AVHRR NDVI data to 
examine vegetation trends over large areas of Canada between 1982 and 1999, and 1982 and 2000, respectively. Both studies found an increasing trend in NDVI in tundra land cover as well as the Prairies and southeastern Ontario. Pouliot et al. (2007) found that $22 \%$ of the vegetated area in Canada showed a significant increase in AVHRR NDVI for the period 1985-2006. In a study of high northern latitudes, Guay et al. (2014) found that AVHRR NDVI increased significantly over $15 \%$ of their study area from 1982 to 2008 , with most of the increases taking place at the higher latitudes and decreasing trends occurring in the lower latitudes.

However, AVHRR was originally designed for weather applications and not vegetation trend studies (Cracknell, 2001) and AVHRR sensor and data processing can introduce noise into the NDVI time series (Steven et al., 2003). Moderate Resolution Imaging Spectroradiometer (MODIS), with higher spatial resolution and better radiometric calibration (Mkhabela et al. (2011), is considered an improvement to AVHRR for more accurately mapping and monitoring vegetation change at regional to global scales. In a study by Fensholt and Proud (2012), MODIS NDVI and AVHRR NDVI time series of an overlapping period were used for evaluating global long-term vegetation trends. They demonstrated an overall good agreement in the spatial distribution of positive and negative NDVI trends, however, MODIS NDVI demonstrated a higher percentage of significant trends in NDVI, especially in the Northern hemisphere. Mkhabela et al. (2011) also compared MODIS and AVHRR and demonstrated that MODIS NDVI outperformed AHVRR NDVI by displaying a higher yield prediction accuracy in evaluating interannual crop changes and crop yield forecasting in the Canadian Prairies during 2000-2006.

MODIS NDVI time series have been widely used for monitoring vegetation trends in different ecosystems. For example, Fang et al. (2018) used MODIS NDVI time series from 2000 to 2011 to monitor vegetation dynamics in Quebec, Canada. They found that over 25\% of the total study area experienced a significant positive or negative trend in NDVI. The negative trends 
were mostly due to abrupt changes from fire, harvest, insect and other disturbances, while the positive trends were due mostly to vegetation plantation growth. Dong et al. (2016) used time series of MODIS FAPAR (Fraction of Absorption Photosynthetically Active Radiation, as a measure of vegetation primary productivity) from 2000 to 2013 to assess the impacts of climate variability on cropland productivity in the Canadian Prairies, finding that it had increased in most areas and that inter-annual variability in cropland productivity was positively related to total annual precipitation variation and negatively related to mean annual temperature. Niti et al. (2015) used MODIS NDVI time series from 2000 to 2014 to detect temporal changes in key greenness metrics in a savanna area in Africa. Most of the area was found to have experienced a statistically significant positive trend, which was associated with woody plant encroachment due to increased moisture and fire control, as well as a delay in start of season, which indicated a shift in vegetation phenology during the period of study. Eckert et al. (2015) used 11-year MODIS NDVI time series to detect land degradation or regeneration in Mongolia. The results showed that $50 \%$ of Mongolia's surface had undergone significant changes that occurred as a result of deforestation, fire, mining, urban expansion and grassland regeneration.

In addition, Landsat data, with lower temporal resolution but much higher spatial resolution and a longer archive from 1973, has been used to monitor long-term vegetation trends in different ecosystem applications such as monitoring change in tundra vegetation (Ju and Masek, 2016), mapping forest dynamics (Maselli, 2004; Potapove et al., 2008; Czerwinski et al., 2014), mapping gradual and abrupt land cover changes due to natural and human-made events (Latifovic, 2005; Eckert et al., 2015; Pouliot and Latifovic, 2016), and assessing grazing impacts on natural grasslands (Svoray et al. 2013; Roder et al., 2008). In addition, the Landsat archive has largely been used for agricultural monitoring and detecting land cover changes in agricultural areas. For example, Bakr et al. (2010) classified Landsat NDVI times series from 1984 to 2008 
to assess land transformation in the West Delta of Egypt and they found a transformation from $100 \%$ barren to $79 \%$ agriculture in this period. Yin et al. (2018) used Landsat time series and a spatial and temporal segmentation technique to detect patterns of agricultural land abandonment in an agricultural region in the Caucasus covering part of Russia and Georgia. They found strong spatial and temporal variation in land abandonment mostly associated with the regional wars and conflicts in the region. Dutrieux et al. (2016) reconstructed historic cultivation cycles in an agricultural region in Brazil using a multi-temporal segmentation technique on Landsat time series of $1984-2015$.

Many studies have compared MODIS and Landsat time series in detecting vegetation change in different ecosystems. For example, Rogers et al. (2017) used MODIS NDVI and Landsat NDVI time series from 2002 to 2014 to detect trends in tree mortality and compare them with the ground data in boreal forests of North America. The results showed a high percentage of negative trends in many areas with Landsat data more consistently identifying trends evident in ground measurements. Latifovic and Pouliot (2014) compared NDVI and NDWI derived from MODIS (2000-2012) and Landsat (1984-2012) time series in evaluation of cumulative long-term changes of undisturbed forest areas in the Athabasca oil sands region. The results showed an average reduction of $18.6 \%$ in NDVI and $31 \%$ in NDWI from 1984 to 2012, while the slope of the decreasing trends increased during the last 10 years or the study, particularly in areas close to mining developments. Potapov et al. (2008) used time series of Landsat TM 5 bands 2-5 and NDVI and MODIS bands 1-7 from 2000 to 2005 to estimate changes in boreal forests. They estimated a loss of $1.63 \%$ in total boreal forest area, where the proportion of identified forest loss was higher in North America compared to Eurasia (5.63\% to 3\%, respectively). They highlighted the precision of aggregate forest cover loss estimation from Landsat data, but also noted the value of MODIS data in efficiently displaying patterns of forest loss. 
This study hypothesized that regional scale inter-annual vegetation analysis of MODIS and Landsat data can be used to monitor vegetation change in farmlands and that inter-annual vegetation dynamics are related to farmland biodiversity. The performance of MODIS and Landsat time series in capturing inter-annual vegetation dynamics and the relationship of trends with biodiversity was compared in this study. Landsat data, with higher spatial resolution, was expected to capture more spatial details and have stronger relationships with biodiversity.

\subsection{Research objectives and questions:}

The objectives of this study were to: 1) determine if farm landscapes of Eastern Ontario experienced inter-annual trends in vegetation phenology and mid-summer productivity or landscape brightness and moisture during the years prior to 2011-2012, and 2) if such trends or temporal variability are related to 2011-2012 biodiversity. MODIS phenological data and midsummer Tasseled Cap components derived from Landsat data were used in the trend analysis. Following this, equivalent datasets (mid-summer NDVI time series) from each sensor were assessed in trend analysis and biodiversity modelling to evaluate the relative effects of spatial resolution on these analyses. The research questions were:

1. Are there inter-annual trends in landscape phenology derived from MODIS time series and is inter-annual variation in phenology related to biodiversity? i.e., Do landscapes with inter-annual trends and/or temporal variability in vegetation phenology have more or less biodiversity?

2. Are there inter-annual trends in mid-growing season vegetation, moisture and brightness indices derived from Landsat time series and are inter-annual variations in these indices related to biodiversity? i.e., Do landscapes with inter-annual trends and/or temporal variability in these variables have more or less biodiversity? 
3. What is the effect of spatial resolution on inter-annual trend detection and biodiversity modelling using temporal metrics? i.e., In comparing mid-growing season NDVI time series derived from MODIS and Landsat, which is better for modelling biodiversity?

This study contrasts with those of Chapters 4 and 5 , which used individual year MODIS and Landsat data, to evaluate relationships of vegetation pehnology and mid summer productivity with farmland biodiversity, respectively. The focus of those chapters was on landscape average and spatial variability in vegetation as analyzed independently for the given years. In this chapter, the focus is on the temporal dynamics of vegetation with respect to long-term trends and interannual variability in both the landscape average and spatial variance of phenology and productivity.

\subsection{Study site and data}

The study area, landscapes and biodiversity data were as described in Chapters 3.1 and 3.2.1. The analyses of this chapter were conducted using gamma diversity for plants, beetles, birds and butterflies and the $3 \times 3 \mathrm{~km}$ landscape extent based on the overall stronger biodiversity models than for the other diversity indices, taxa and the $1 \times 1 \mathrm{~km}$ extent as found in Chapters 4 and 5 . The MODIS weekly composite NDVI data and derived phenological variables were described in Chapters 3.2.2.1 and 4.4.1. For this study, to represent average mid-summer productivity, the averages of July MODIS data for the period 2000-2012 were used. The Landsat data were described in Chapters 3.2.2.2. and 5.4 and the mid-summer years and dates of cloud free acquisitions for the period 1985 to 2011 were given in Table 5.1.

\subsection{Methods}

Figure 6.1 outlines the main processes and methods used in this chapter. A detailed description of each step follows. 


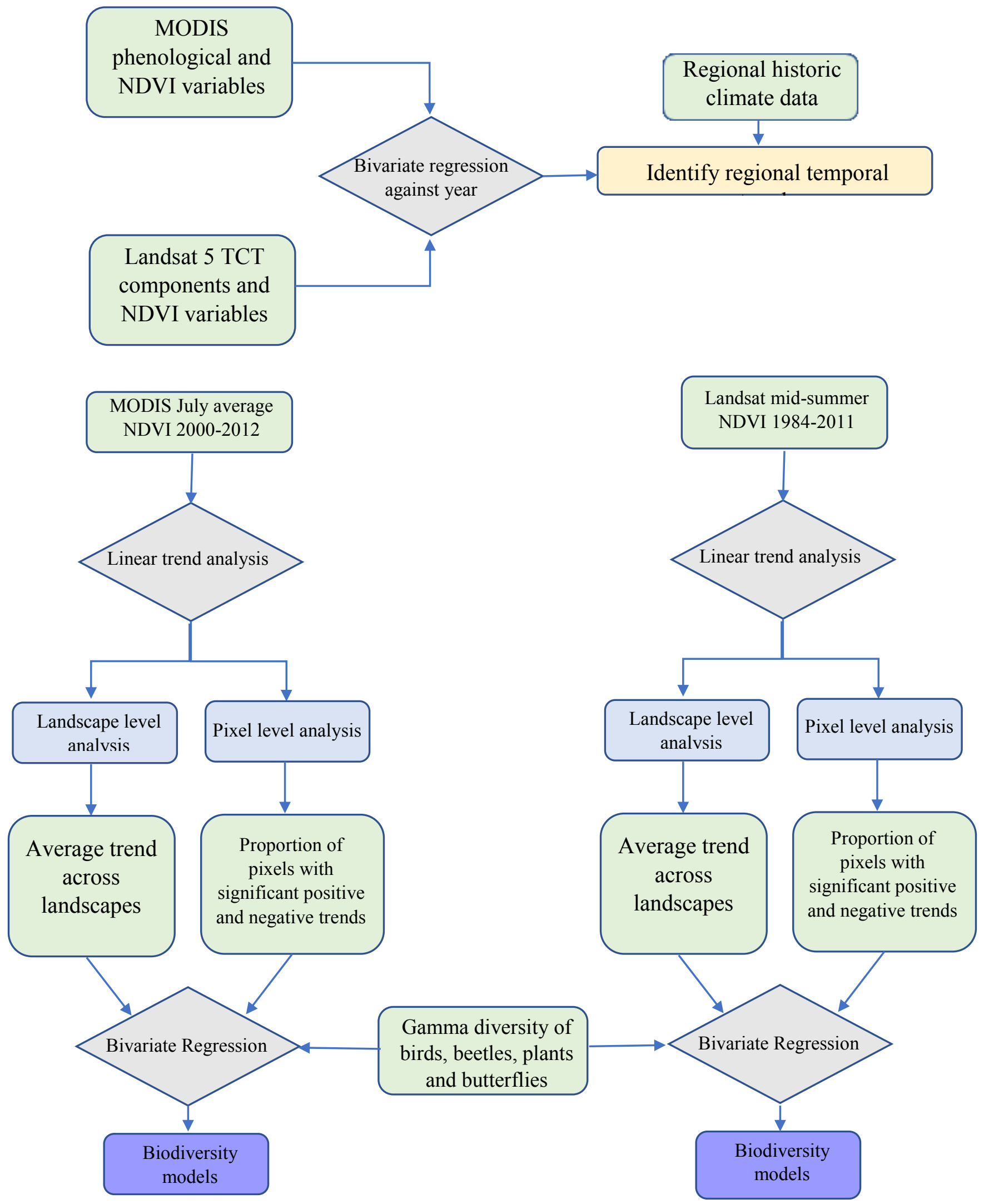

Figure 6. 1. Flow chart outlining various steps used in this chapter. This includes linear trend analysis of MODIS and Landsat NDVI time series and modeling the temporal changes against gamma diversity for the selected taxa. 


\subsubsection{Exploratory trend analysis using time series of MODIS phenology metrics and}

Landsat mid-summer NDVI and TCT components: Regional scale

Inter-annual trend analysis was conducted using remote sensing data from the years preceding the biodiversity data collection. MODIS phenology data (2000-2012) and mid-summer Landsat (1985-2011) NDVI and TCT components were selected to correspond to the data in Chapters 4 and 5, respectively. The trends in these variables were first tested over the whole study region of Eastern Ontario, where the variables for each sensor were averaged over all $933 \times 3 \mathrm{~km}$ landscapes for each year and were plotted independently against time. Most did not show nonlinear trends, hence, linear trend analysis was performed on both data sets. Identified trends were assessed in relation to historic climate data to determine if the latter may have had an effect on the inter-annual landscape dynamics.

\subsubsection{Comparison of MODIS and Landsat mid-summer NDVI trends: Landscape scale}

MODIS phenological variables and Landsat TCT components were not used in further analysis of this chapter as these variables were not calculated for both datasets and could not be used to compare the detected vegetation trends by each sensor and the capability of the time series of each dataset in capturing landscape trends. To compare MODIS and Landsat NDVI trend analysis in more depth, mid-summer MODIS and Landsat NDVI time series were used. Mid-summer NDVI was selected as an index common to both sensors. This analysis was conducted to assess the inter-annual trends of MODIS and Landsat time series at two levels:

1) the landscape level by comparing landscape-averaged NDVI with respect to the compositional and configurational heterogeneity of the sample landscapes as explained in Chapter 3.1 (high compositional, high configurational $\left(\mathrm{H}_{-} \mathrm{H}\right)$, high compositional, low configurational (H_L), low compositional, high configurational (L_H) and low compositional, low configurational (L_L). 
2) the pixel level by calculating the proportion of pixels in each sample landscape with significant positive and negative trends and the proportion showing no significant trend.

As stated above, for MODIS, the average NDVI for all July weekly composites was used while for Landsat the best July scene (less than 5\% cloud cover) was selected. To examine the effect of the length of the time series on trend analysis, MODIS data for 2013-2015 (i.e. after the biodiversity data collection years) were added to the 2000-2012 time series, while for Landsat the 1985-2011 temporal period was split into two sub-periods (1985-2000 and 2000-2011) and trends were modelled for each. The Landsat sub-periods were selected based on an observed change in direction and/or magnitude of slope of average NDVI in some sample landscapes. Furthermore, the later period overlapped with the available MODIS NDVI time series and enabled testing of the relative effect of spatial resolution of MODIS and Landsat NDVI on the trends and subsequent biodiversity modelling.

The Theil-Sen (TS) slope linear trend measure (Theil 1950, Sen, 1968) was selected to compute trend magnitudes since it avoids spatial and temporal autocorrelation. TS estimates the median of all $n(n-1) / 2$ slopes calculated between observation values at all pair-wise time steps (Parmentier et al., 2014; Latifovic and Pouliot, 2014). It is a noise resistant metric that offers simplicity in computation, provides analytical estimates of intervals and is robust to outliers. It includes assumptions regarding residuals that are testable and requires limited a priori information regarding measurement errors (Latifovic and Pouliot, 2014). The robustness of the TS technique enables it to reject outlier values without affecting the slope. According to Hoaglin et al. (2000) approximately $29 \%$ of the outlier values in a sample may be rejected by this method without affecting the slope. 
Although TS slope is a robust trend measure, it does not take into account the significance of trends, which is hard to establish due to the increased variance from that noise (Neeti and Eastman, 2011). To address this, the Contextual Mann-Kendall (CMK) test developed by Neeti and Eastman (2011) to assess the significance of trends was used. The null hypothesis is that no trend exists; i.e., the data are randomly ordered and are not time-dependent. The principle of this method is to characterize ground features based on the spatial autocorrelation, presuming that a pixel would not show a drastically different trend from neighboring pixels (Latifovic and Pouliot, 2014; Czerwinski et al., 2014). If an isolated location indicates a trend while none of its neighbouring locations do, it is expected that the trend in spurious. The addition of contextual information would be helpful in removing such trends and reinforcing the evidence when adjacent pixels are trending similarly. The $\mathrm{CMK}$ test removes serial correlation (related to temporal autocorrelation) and adjusts the neighbouring pixels for cross-correlation (related to spatial autocorrelation), while combining their information (Neeti and Eastern, 2011).

The CMK procedure tests the slopes between all pair-wise combinations of samples. The data are referenced to time and each data point is the reference to the next data point in the sequential time period (Neeti and Eastman, 2011). Furthermore, it directs all n(n-1)/2 iterations in a way that, if the value of a later data point is higher than an earlier one (the slope is positive), then the $\mathrm{S}$ statistic increases by 1 and if the opposite is true, it decreases by 1 (Schlagel and Newton, 1996). Kendall $\mathrm{S}$ is defined as:

$s=\sum_{i=1}^{n-1} \sum_{j=i+1}^{n} \operatorname{sign}\left(x_{i}-x_{j}\right)$

(Equation 6.1)

where

$\operatorname{sign}\left(x_{i}-x_{j}\right)=\left\{\begin{array}{c}1 \text { if } x_{i}-x_{j}<0 \\ 0 \text { if } x_{i}-x_{j}=0 \\ -1 \text { if } x_{i}-x_{j}>0\end{array}\right.$ 
$\mathrm{n}$ is the length of the time series and $\mathrm{x}_{\mathrm{i}}$ and $\mathrm{x}_{\mathrm{j}}$ are the observations at times $\mathrm{i}$ and $\mathrm{j}$, respectively. The variance of the $\mathrm{S}$ statistic, in relation to $\mathrm{n}$ is used to determine a significance (p) value, which indicates the probability of obtaining a value greater than or equal to $\mathrm{S}$, when no trend is present. Details of integrating TS and CMK test can be found in Neeti and Eastman (2011).

The TS slope and CMK significance of trends for MODIS and Landsat vegetation index time series were calculated for each $3 \times 3 \mathrm{~km}$ sample landscape. Trends were expressed by calculating the percentage of pixels in each landscape that demonstrated a significant positive and negative slope $(p \leq 0.05)$ or no statistically significant slope $(p>0.05)$. The average of NDVI over all pixels within each landscape was then calculated for each year and the resulting trends were evaluated to determine if landscape scale vegetation indices increased or decreased during the period of study.

The magnitude and direction of TS slopes were also compared for each sample landscape based on their composition and configurational heterogeneity to determine if vegetation trends varied among more heterogeneous versus more homogenous landscapes.

\subsubsection{Biodiversity modeling}

To assess the relationships of vegetation trends and temporal variability with biodiversity, linear correlation analysis was used. The percentage of pixels with negative, positive and no trends, as well as the coefficient of variation $(\mathrm{CV})$ of the landscape spatial average and $\mathrm{CV}$ in inter-annual NDVI over the time period for each sample landscape were modeled against the gamma diversity of birds, beetles, butterflies and wild plants. Models created using MODIS and Landsat NDVI trend and variability data were then compared. In addition, for taxa showing significant relations with both types of explanatory variables (percentage of pixels with significant positive and negative trends and the coefficient of variation of NDVI) stepwise multiple regression (SWR) 
was implemented using both types of predictors as input variables to determine if stronger models could be generated and the relative contributions of the variables to the models.

\subsection{Results}

\subsubsection{Exploratory trend analysis using time series of MODIS phenology metrics and Landsat mid-summer NDVI and TCT components: Regional scale}

\subsubsection{MODIS}

Figure 6.1 shows the MODIS-derived phenological variables for the period 2000-2012, averaged over all $3 \times 3 \mathrm{~km}$ sample landscapes and plotted with $95 \%$ confidence intervals. Mean EOS NDVI was the only significant variable $(p=0.03)$ demonstrating a decreasing trend with a maximum of 0.63 (in 2004) and a minimum of 0.56 (in 2012). However, it was noted that the trend was affected by the low end-point of the time series (2012) and it became weaker and statistically insignificant $(p=0.16)$ when 2012 was removed and 2011 was considered as the end point. Despite this, Mean EOS NDVI was selected for more detailed TS slope and CMK significance analysis and used in modelling against biodiversity.

Some other phenological variables demonstrated a slight negative or positive slope that was almost significant. For example, Mean MAX DOY $(r=-0.49 ; p=0.08)$ varied from a maximum of 219 in 2000 to a minimum of 206 in 2012 and MAX DOY CV $(r=-0.53 ; p=0.06)$ varied from a maximum of 0.043 in 2001 to a minimum of 0.030 in 2011 . These results indicate that the date of peak vegetation productivity may be getting earlier while the variability across landscapes of the date of peak productivity may be decreasing as farmlands become more uniform in timing of cropping and practices. The average date of onset of greenness (Mean SOS DOY) and senescence or harvest (Mean EOS DOY) demonstrated a decrease over the study period but the data were highly variable and the estimated decrease was only about 2 days for both, resulting in models that were not close to being significant. The spatial variability of NDVI at start of 
season (SOS NDVI CV) showed an increase during this time period, with a range from a minimum value of 0.11 (2005) to a maximum value of 0.15 (2012), although the model was far from significant and the data appear to be curvilinear. Other non-significant models that showed curvilinearity or had points at or near the ends of the series that were distinctly higher or lower than most points included Mean MAX NDVI, MAX NDVI CV, and MAX DOY CV.

To test the effect of time series length, MODIS phenological variables for 2013, 2014 and 2015 were added to the 2000-2012 time series. The significance of trends with these additional years did not increase and the length of the time series did not have a significant effect on the slope of trend lines.

Historic climate data (Table 6.1) were used to evaluate the effects of weather on the observed trends. An average of three Environment and Climate Change Canada climate stations (Russell, Cornwall and St. Albert stations, Environment and Climate Change Canada, 2018) was used to look at the climate variation across the region encompassing the sample landscapes. A significant positive relationship (Figure 6.3; $\mathrm{r}=0.52 ; \mathrm{p}=0.05$ ) between Mean SOS DOY and the first DOY with no snow on the ground was found. For example, in 2012 with the earliest SOS DOY in the time series (DOY 121) the first DOY with no snow was March 14 (DOY 73), while in 2009 with the latest SOS DOY in the time series (DOY 131) the first day with no snow was April 16 (DOY 106). Consequently, vegetation productivity in the years with earlier SOS DOY reached its peak (Max DOY) and senescence (EOS DOY) at an earlier time. For example, 2012 demonstrated the earliest Mean MAX DOY and the second earliest Mean EOS DOY in the time series. In addition, total July precipitation was expected to have an effect on the average NDVI at the time of vegetation peak and senescence. For example, 2012 had the lowest total July precipitation $(27.1 \mathrm{~mm})$ compared to the $2000-2012$ average $(86.5 \mathrm{~mm})$, and it demonstrated the lowest Mean Max NDVI and Mean EOS NDVI in the time series. The year 2001 had the second 
lowest total annual precipitation (49 mm) and its Mean MAX NDVI was as low as 2012. The average July temperature varied from a minimum of $19.7^{\circ} \mathrm{C}$ in 2000 to a maximum of $22.9{ }^{\circ} \mathrm{C}$ in 2012. Although it was not significantly correlated with any of the phenological variables, the extreme value of 2012 was deemed to affect the phenological events in that year.

The above analysis of MODIS phenological variables along with the investigation of annual climate data revealed that extreme weather events in 2012 (early snow melt and dry July), could create potential bias if used as the end point of the times series. For this reason, and because Landsat data were only available to 2011, the 2012 MODIS data were removed from further analysis.

\subsubsection{Landsat}

Figure 6.4 shows the mid-summer Landsat-based TCB, TCG, TCW, and NDVI data averaged for all $3 \times 3 \mathrm{~km}$ sample landscapes and plotted with 95\% confidence intervals for each year of data to evaluate their inter-annual variability (Figure 6.4). A slight positive or negative slope was evident for all variables, however, the trends were not statistically significant $(\mathrm{p}>0.05)$. Relatively high r-values and low p-values for Mean TCB and TCB CV suggested nearly significant trends $(r=0.42, p=0.08$ and $r=0.47, p=0.06$, respectively). Mean TCB decreased over the 1985 to 2011 period, while TCB CV increased. This suggests that the area of bare soil in the landscapes declined and more vegetation was present, perhaps due to increased cropping area or to more dense crops at the time of data acquisition. Mean and TCG CV showed weak declining trends $(\mathrm{r}=0.34, \mathrm{p}=0.16$ and $\mathrm{r}=0.14, \mathrm{p}=0.5$ respectively). However, the trend in TCG CV would be statistically significant $(\mathrm{r}=0.54 ; \mathrm{p}=0.03)$ if the year 2011 was removed as the end point in the series as it was $27 \%$ greater than the next highest value and the other values only demonstrated a difference of 0.1 . Mean TCW showed a weak increasing trend $(r=0.32, p$ $=0.19$ ) while TCW CV showed no discernable trend. 
Table 6.1. The average of climate data for three Environment and Climate Change Canada weather stations (Russell $\left(45^{\circ} 15^{\prime} 46.008^{\prime \prime} \mathrm{N}, 75^{\circ} 21^{\prime} 34.032^{\prime \prime} \mathrm{W}\right)$, Cornwall $\left(45^{\circ} 00^{\prime} 56.082^{\prime \prime}\right.$

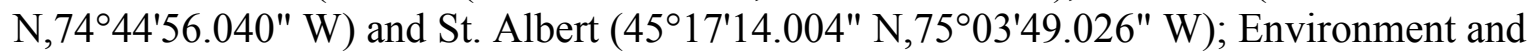
Climate Change Canada, 2018).

\begin{tabular}{|c|c|c|c|}
\hline Year & $\begin{array}{c}\text { First DOY with no } \\
\text { Snow on the Ground }\end{array}$ & $\begin{array}{c}\text { Total July } \\
\text { Precipitation }(\mathrm{mm})\end{array}$ & $\begin{array}{c}\text { Mean July } \\
\text { Temperature }(\circ \mathrm{C})\end{array}$ \\
\hline 1985 & 11-Apr (DOY 101) & 81.2 & 20.4 \\
\hline 1987 & 27-Mar (DOY 86) & 114.0 & 21.6 \\
\hline 1994 & 19-Apr (DOY 109) & 54.1 & 21.7 \\
\hline 1995 & 9-Apr (DOY 99) & 149.6 & 21.8 \\
\hline 1996 & 25-Apr (DOY 115) & 183.6 & 20.0 \\
\hline 1997 & 15-Apr (DOY 105) & 74.13 & 20.4 \\
\hline 1998 & 29-Mar (DOY 88) & 97.1 & 20.6 \\
\hline 2000 & 15-Apr (DOY 105) & 118.6 & 19.7 \\
\hline 2001 & 10-Apr (DOY 100) & 49.0 & 19.7 \\
\hline 2002 & 1-May (DOY 121) & 64.9 & 21.7 \\
\hline 2003 & 10-Apr (DOY 100) & 102.2 & 20.9 \\
\hline 2004 & 7-Apr (DOY 97) & 112.1 & 20.8 \\
\hline 2005 & 1-Apr (DOY 91) & 79.2 & 22.0 \\
\hline 2006 & 24-Mar (DOY 83) & 147.0 & 22.4 \\
\hline 2007 & 24-Mar (DOY 83) & 146.5 & 20.2 \\
\hline 2008 & 12-Apr (DOY 102) & 72.1 & 20.3 \\
\hline 2009 & 16-Apr (DOY 106) & 113.5 & 19.8 \\
\hline 2010 & 13-Mar (DOY 72) & 49.0 & 22.8 \\
\hline 2011 & 24-Mar (DOY 83) & 51.2 & 22.7 \\
\hline 2012 & 14-Mar (DOY 73) & 27.1 & 22.9 \\
\hline & & & \\
\hline
\end{tabular}




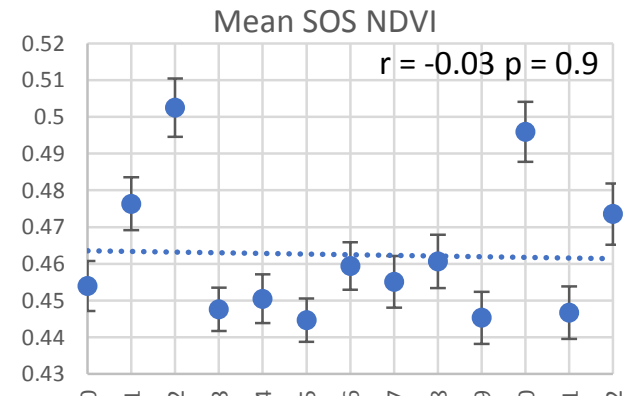

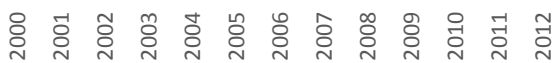
SOS DOY CV
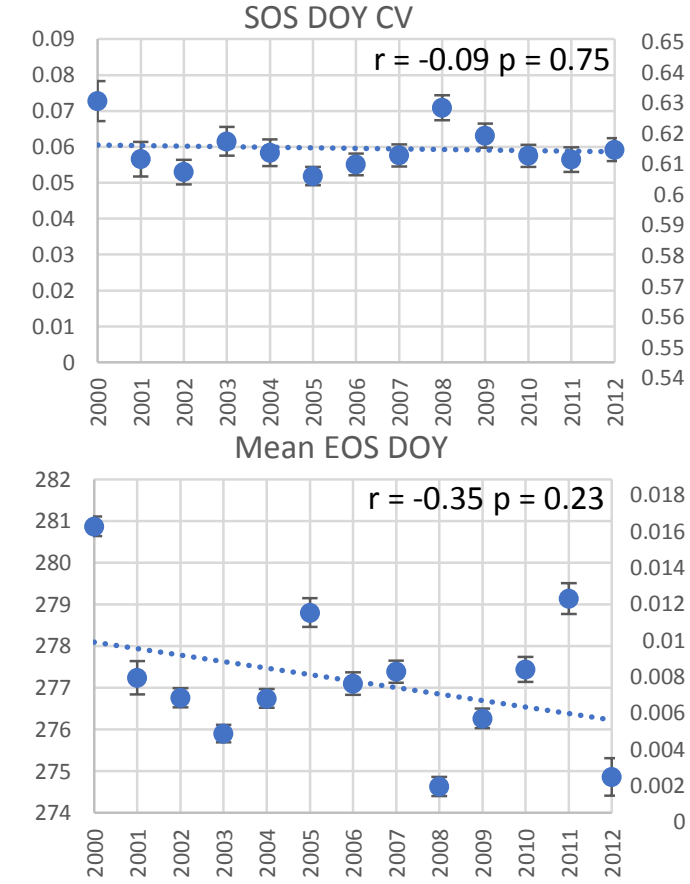

MAX NDVI CV

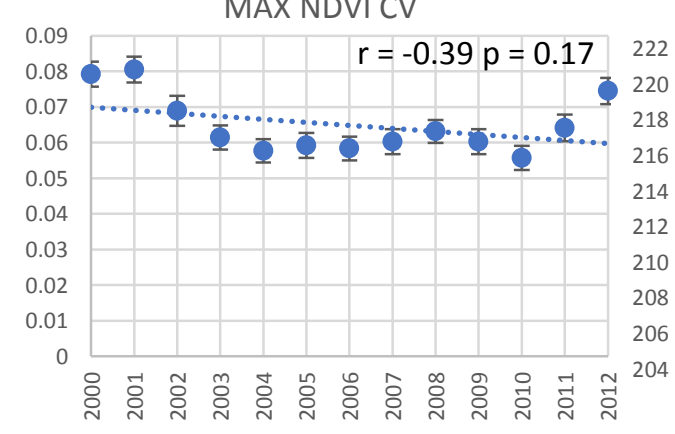

Mean SEAS GRN

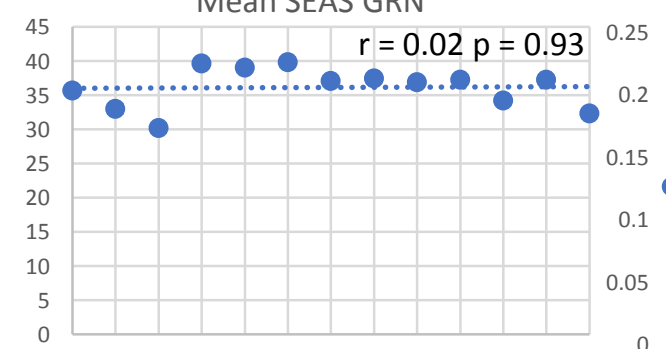

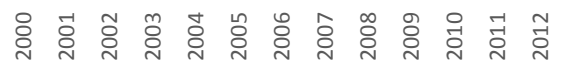

SOS NDVI CV

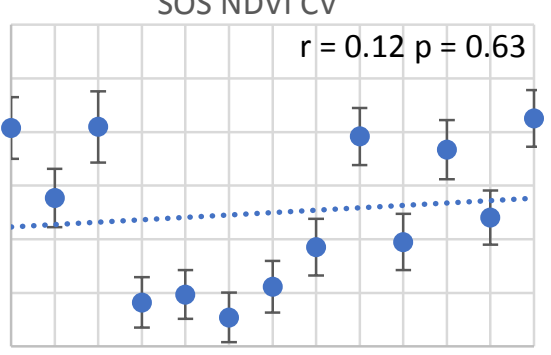

o o Mean EOS NDVI

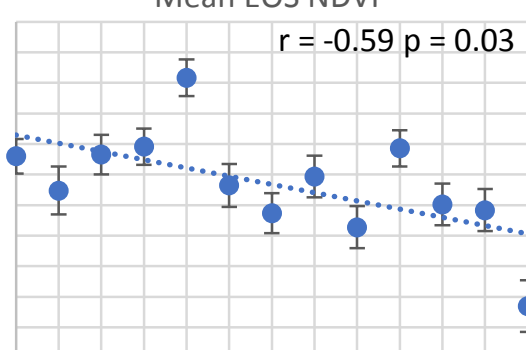

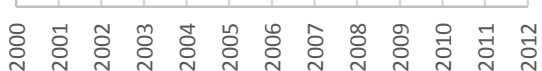
EOS DOY CV

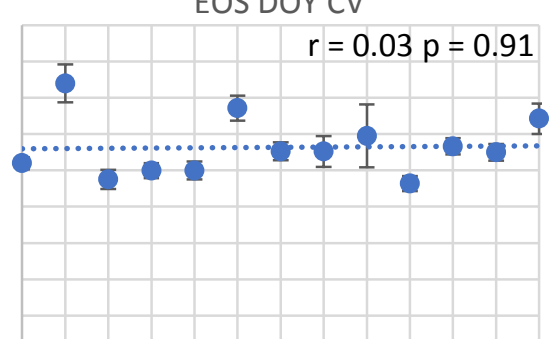

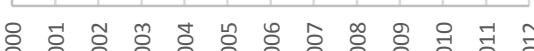
Mean MAX DOY

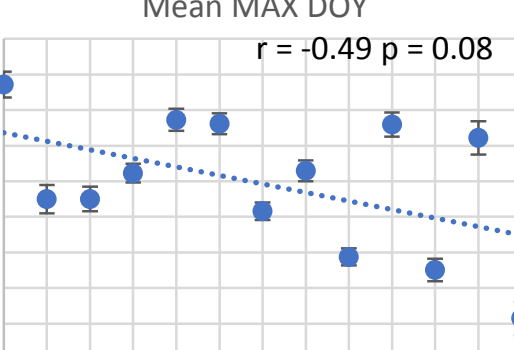

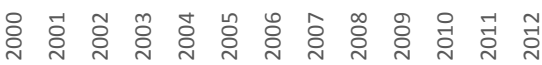
SEAS GRN CV

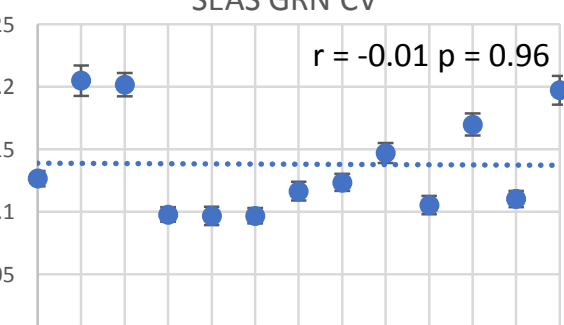

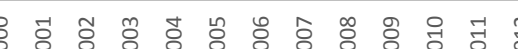

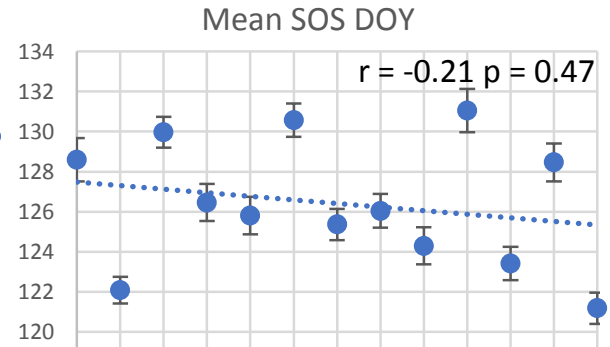

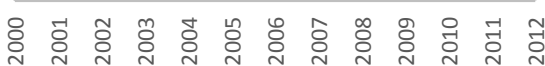
EOS NDVI CV

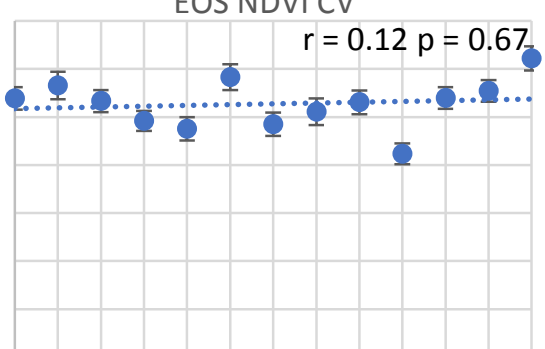

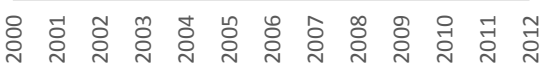
Mean MAX NDVI

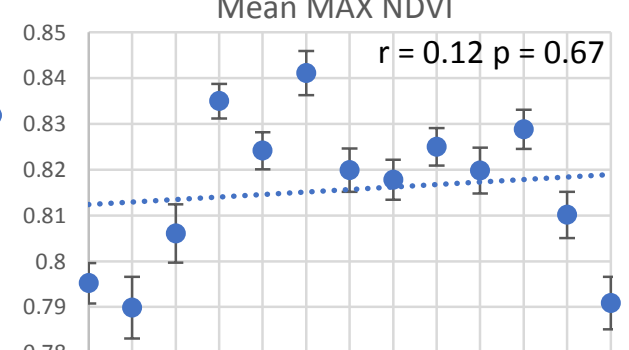

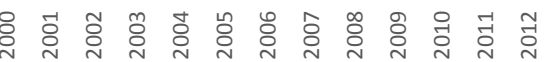
MAX DOY CV

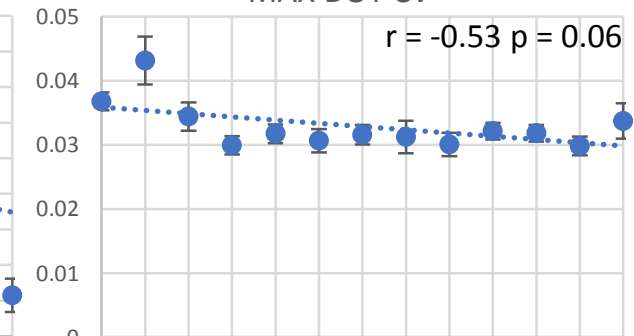

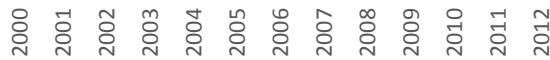
Mean SEAS AMP

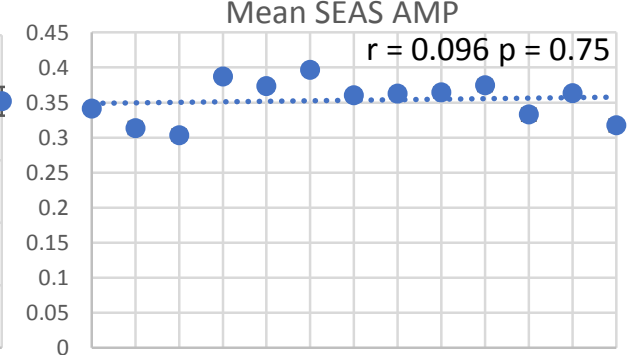

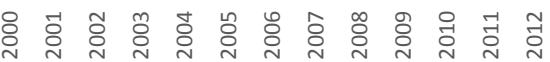



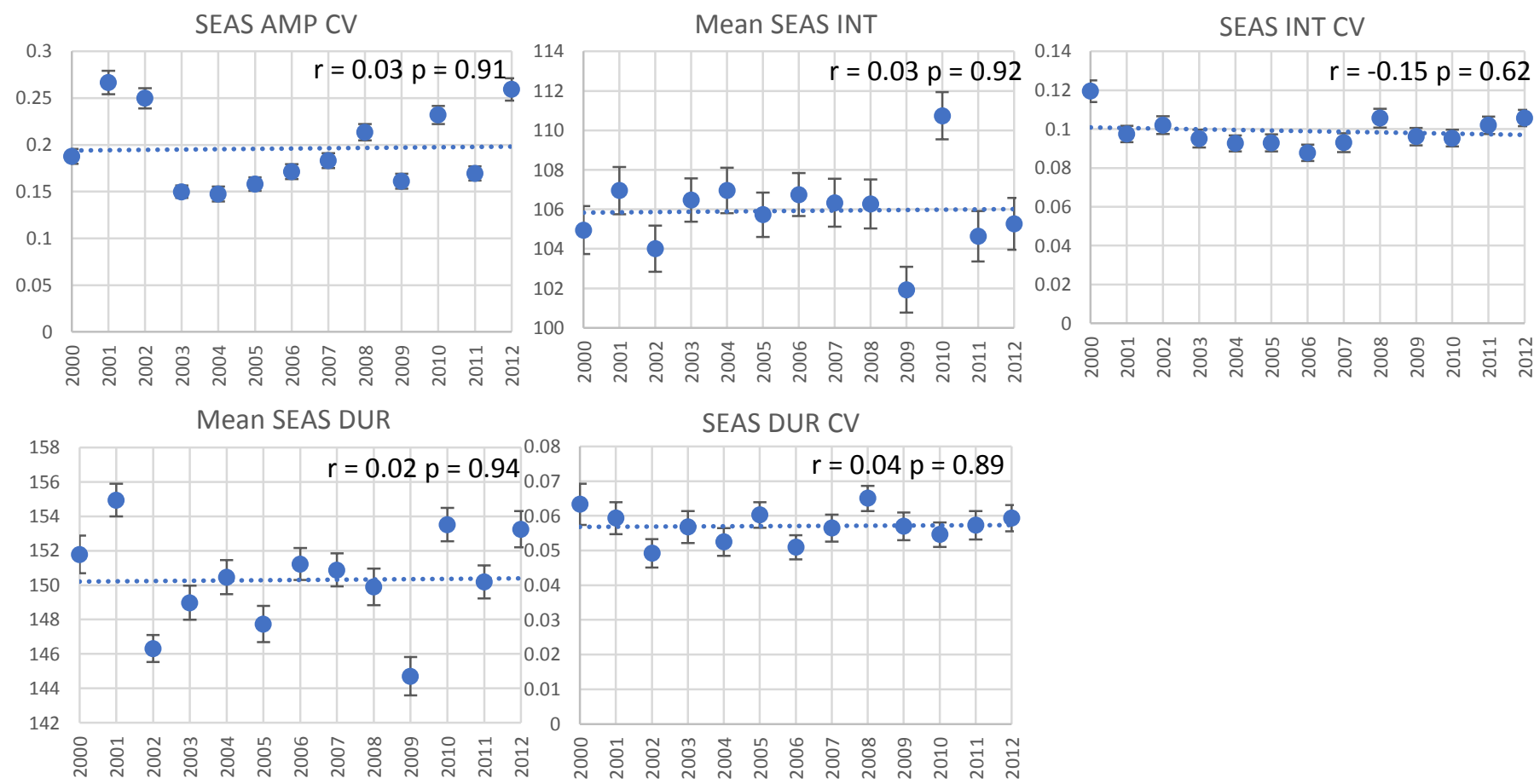

Figure 6.2. Inter-annual variability and linear trends of MODIS-derived phenological variables averaged over all $3 \times 3 \mathrm{~km}$ sample landscapes $(\mathrm{N}=93$, bars show \pm 2 standard deviations).

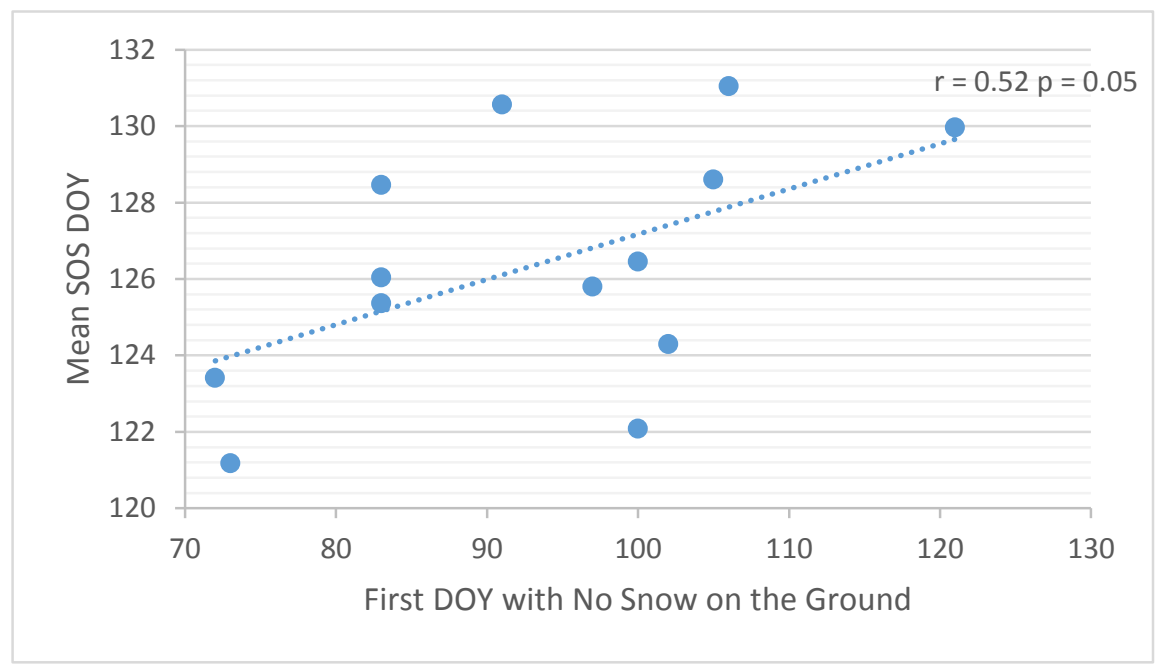

Figure 6.3. The relationship between Mean SOS DOY and the first DOY with no reported snow on the ground.

Although no overall significant correlation of TCT components with the climate data (Table 6.10) was found over the years, they demonstrated association with the extreme weather events. For example, high Mean TCW (1995 and 2007) was deemed to be associated with higher 
total July precipitation, while low Mean TCW (2001 and 2008) was associated with low total July precipitation. Low Mean TCG, low Mean NDVI and high NDVI CV in 2001 were found to be associated with low total July precipitation (lowest on the 28-year record), while high Mean TCG and relatively high Mean NDVI in 2007 were associated with high total July precipitation. No clear pattern was found between Mean TCB and the climate parameters.

Furthermore, low within-year variability of the predictors, indicated by the $95 \%$ confidence bounds (2 standard deviations) as shown in Figure 5.8, demonstrated that the predictors' range did not vary much among the sample landscapes in a given year. Only TCB CV showed higher variability between the sample landscapes and more consistently so in later years.

\subsubsection{Comparison of MODIS and Landsat mid-summer NDVI trends: Landscape scale}

\subsubsection{MODIS}

\subsection{Landscape heterogeneity analysis}

Average NDVI for most of the sample landscapes showed no significant trends during 20002011. Figure 6.5 shows examples for H_H, L_L, H_L and L_H sample landscapes. However, eight sample landscapes demonstrated a slight increase $(0.02-0.03)$ in average NDVI $(\mathrm{p} \leq 0.05$; examples shown in Figure 6.6). Of these eight landscapes, two were H_H, three H_L, one L_H, and two L_L; i.e., there was no pattern with respect to the landscape heterogeneity categories when landscape averaged NDVI was used in the trend analysis. These small changes in average NDVI over the years were negligible and within the noise range of the data and may not be representative of change in vegetation productivity 

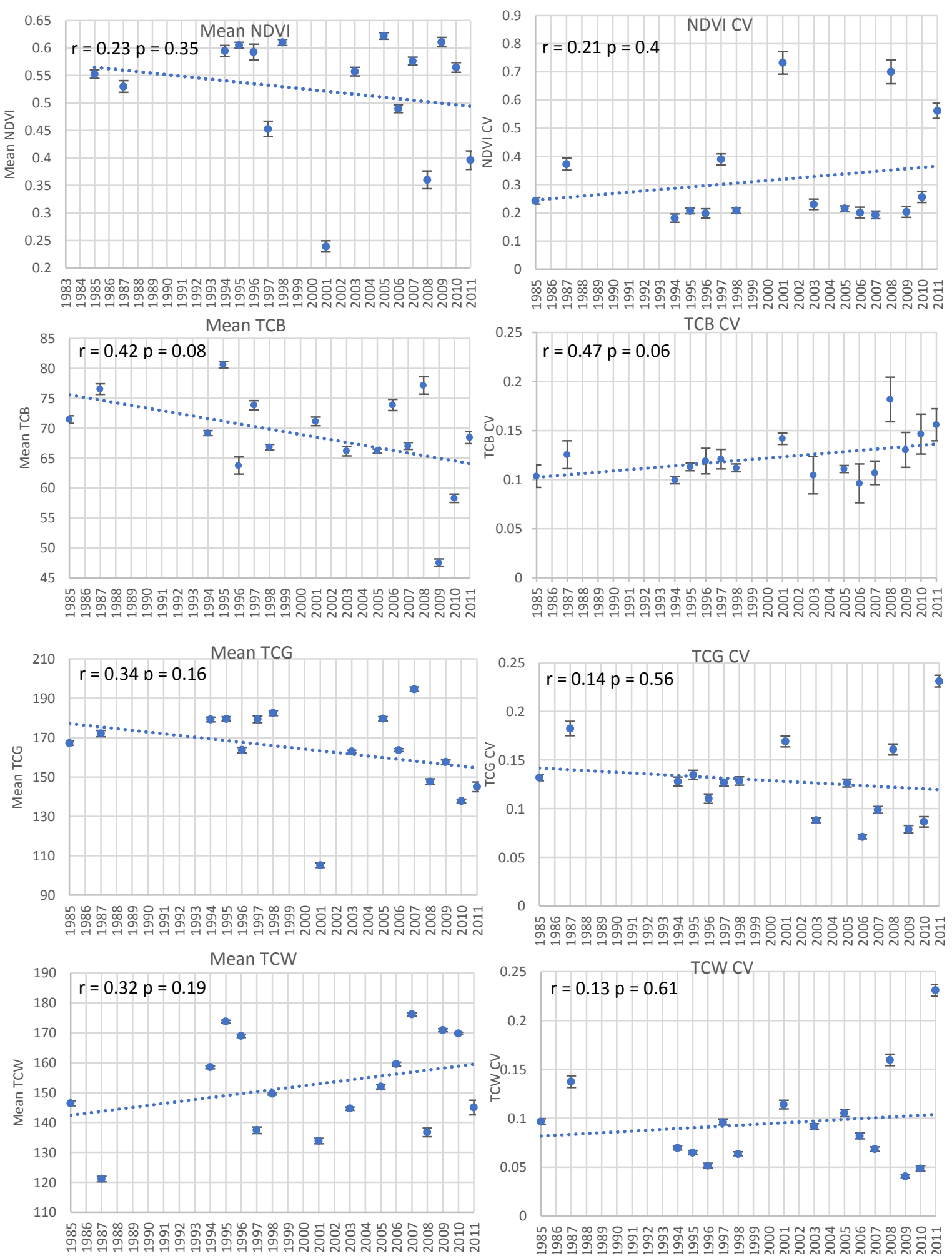

Figure 6.4. Inter -annual variability and linear trends of Landsat-derived mid-summer variables averaged over all $3 \times 3 \mathrm{~km}$ sample landscapes $(\mathrm{N}=93$, bars show \pm 2 standard deviations). 

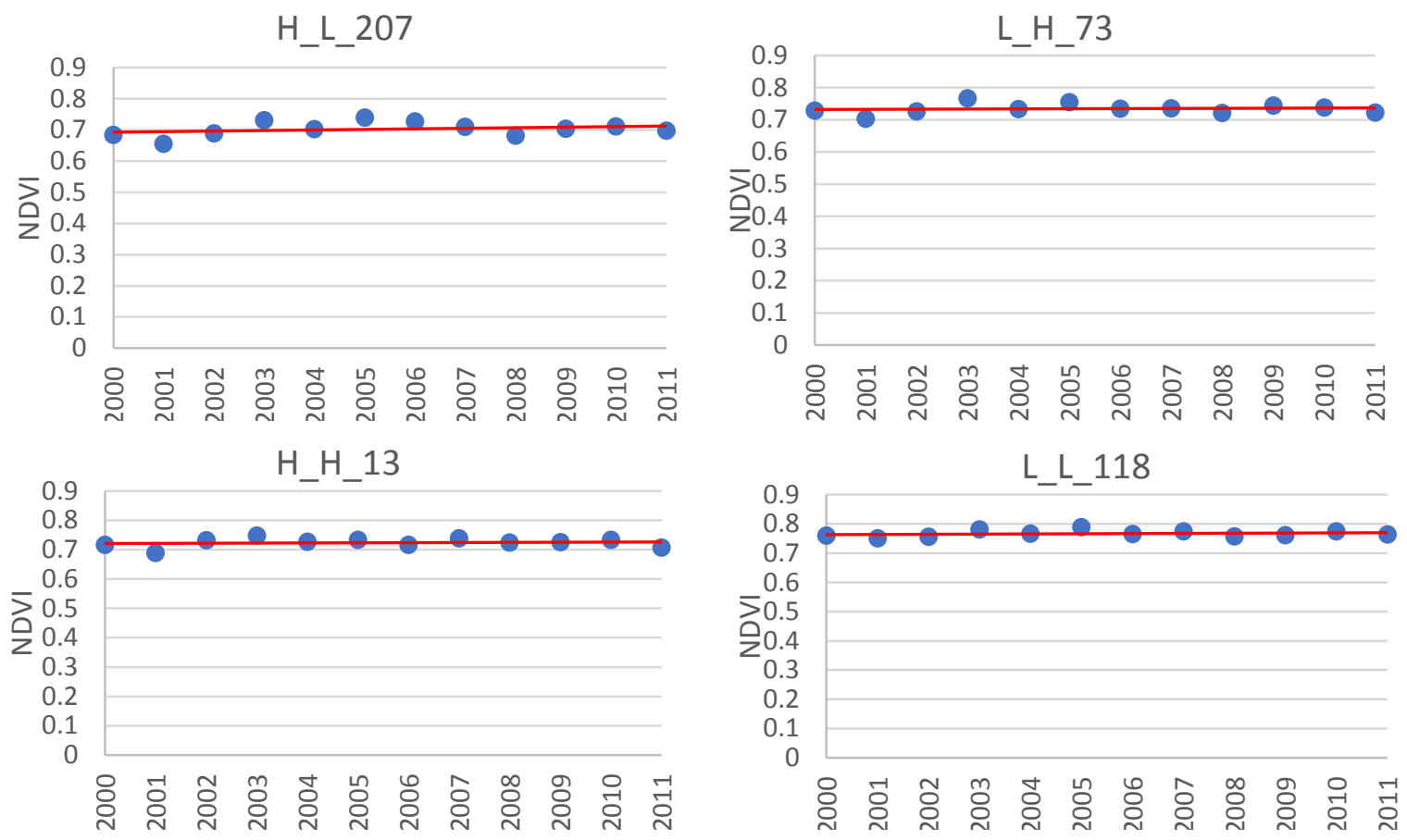

Figure 6.5. Examples of H_H, L_L, H_L and L_H sample landscapes with no significant MODIS mid-summer NDVI trend (spatial average of NDVI across each sample landscape, $\mathrm{p}>$ $0.05)$.
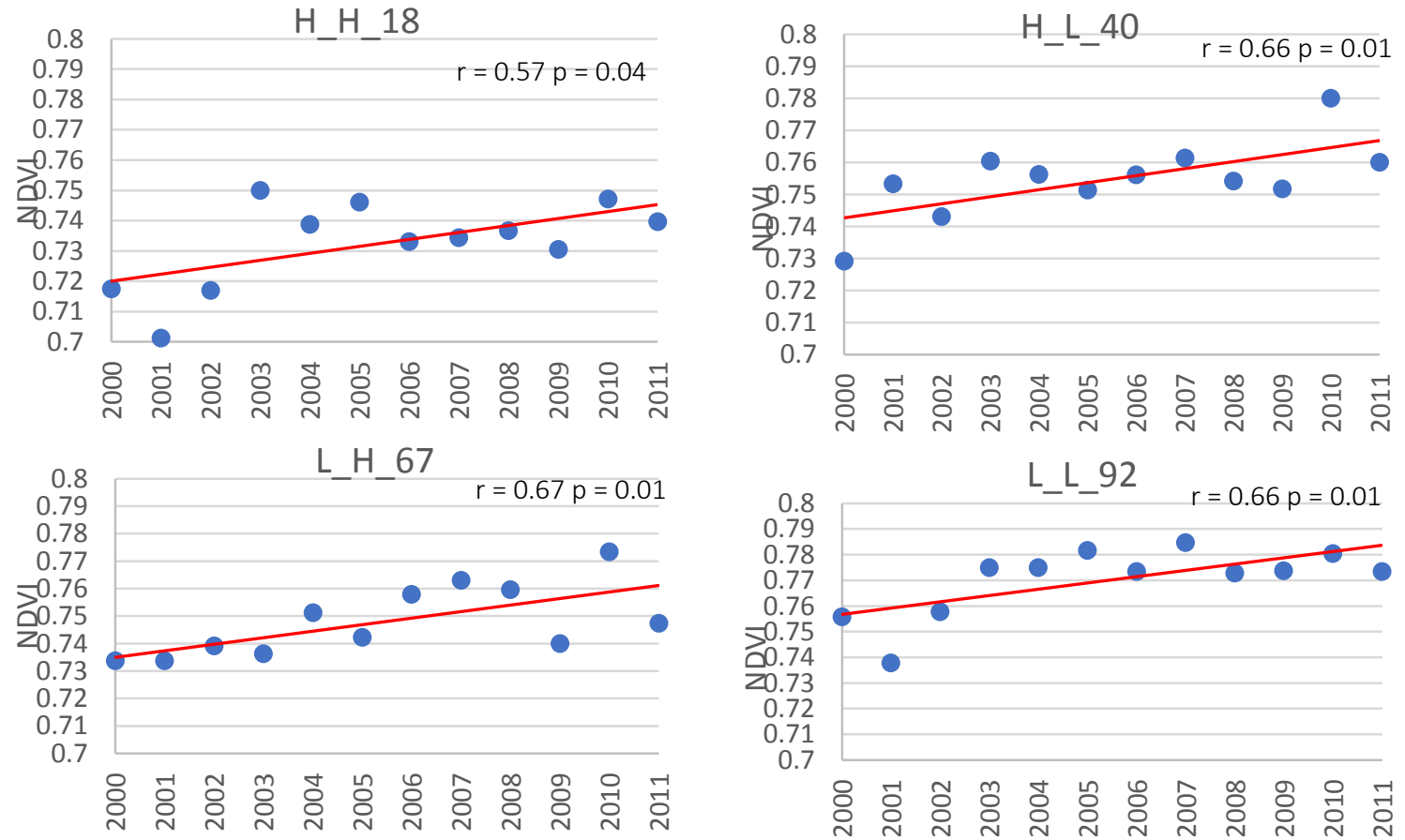

Figure 6.6. Examples of H_H, L_L, H_L and L_H sample landscapes with a significant positive MODIS mid-summer NDVI trend (spatial average of NDVI across each sample landscape, $\mathrm{p} \leq$ $0.05)$. 
Analyzing the magnitude and direction of trends with respect to landscape heterogeneity and without considering trend significance showed that the majority of all landscapes had positive trends (Figure 6.7). More L_L landscapes showed decreasing NDVI, but as few relationships were significant, this pattern cannot be conclusively stated to be indicative of landscape heterogeneity effects.

\subsection{Pixel level analysis}

The TS slope for the July average of MODIS NDVI was calculated for 2000-2011 at the pixel level within each landscape. Figure 6.7 shows pixels with a positive TS slope and an increasing trend in vegetation productivity and/or biomass displayed in green, while pixels presented in red had a negative TS slope, indicating a decreasing vegetation trend. Note that all positive or negative slope values are shown regardless of statistical significance. Pixels presented in white display a TS slope of 0.00 and indicate areas with no change in vegetation productivity.

The significance of these TS slopes $(\mathrm{p} \leq 0.05)$ was tested using the CMK test; Figure 6.8 shows the areas with significant positive and negative trends using the same colour scheme as for Figure 6.8. Comparing the TS slope and CMK significance maps showed that, although many pixels demonstrated positive or negative TS slope, most of these slopes were not of statistical significance. Over all sample landscape pixels (93 landscapes $\times$ (12 to 16$)$ pixels/landscape $=$ 1,116-1,488 total pixels), at least $62 \%$ of the pixels demonstrated no significant trend. In 69 landscapes $0-5 \%$ of pixels demonstrated significant negative trends, while in 18 landscapes 5$10 \%$ of pixels demonstrated significant negative trends. Of these 18 landscapes, only two were H_H, while seven were L_L. In six other landscapes, four of which were L_L, more than $10 \%$ of all pixels were negatively trended. In addition, 31, 23 and 39 sample landscapes had 0-5\%, 5$10 \%$, and more than $10 \%$ of pixels, respectively, with significant positive trends, with the highest percentage being 66\% for L_H_80. Of the 39 sample landscapes with more than $10 \%$ of pixels 
with significant positive trends, $16(41 \%)$ were H_H, while only five $(13 \%)$ were L_L. Table 6.2. gives the proportion of significant negative and positive pixels with regards to the landscape heterogeneity groupings. It indicates that, although the proportions of pixels in landscapes with significant positive or negative trends were generally small, the area of landscapes showing significant trends may be related to landscape heterogeneity. Larger areas of low heterogeneity landscapes showed significant negative NDVI trends while larger areas of high heterogeneity landscapes showed significant positive trends. It also showed that the proportion of positively trended pixels for all landscape heterogeneity groupings was much larger compared to the proportion of negatively trended pixels.

The CMK significance image was compared with the AAFC annual crop inventory map (2012) and high-resolution satellite imagery to determine the land cover classes associated with significant positive and negative trending pixels. Most of the positive trends occurred in the cropped portion of the sample landscapes, while most of the negative trends were associated with wooded and urban areas. This demonstrates the potential impacts of agricultural practices in increasing crop yield and productivity over time, while showing that removal of natural (woody) vegetation and creation of new structures or bare surfaces have taken place in the study landscapes in the period 2000-2011. Figure 6.10 displays examples of significant positive and negative trends in inter-annual mid-summer MODIS NDVI. 


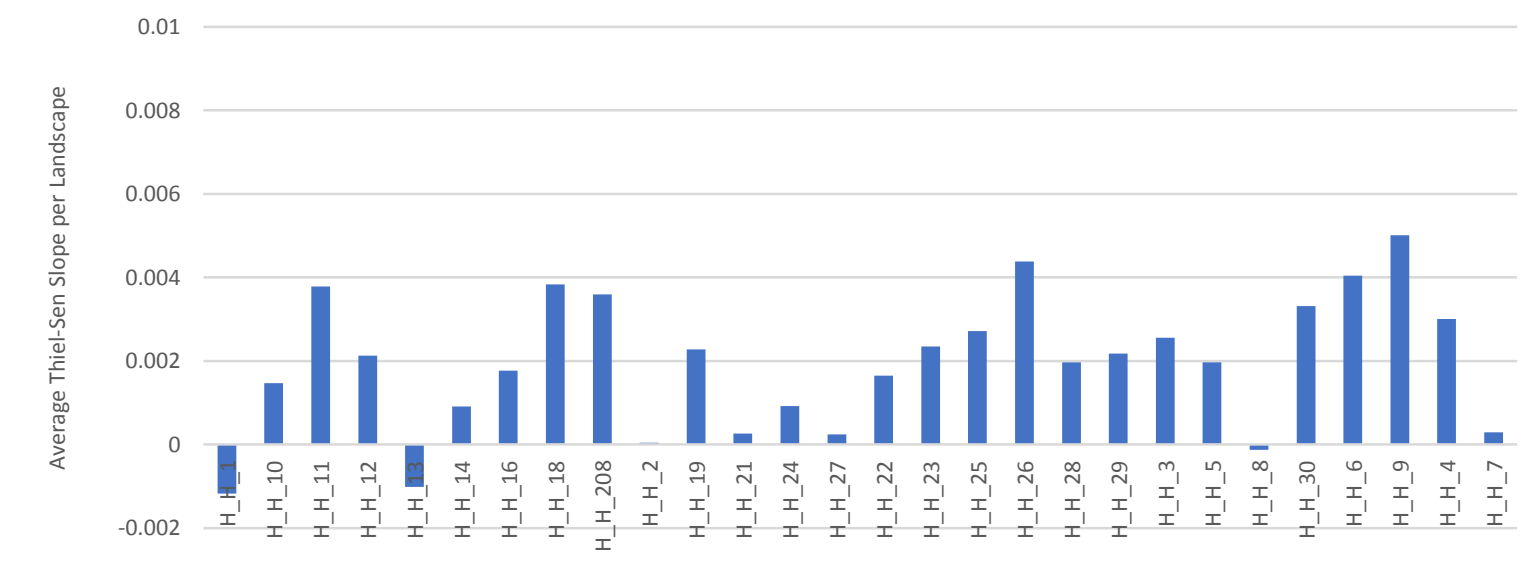

b

TS Slopes for H_L and L_H Landscapes

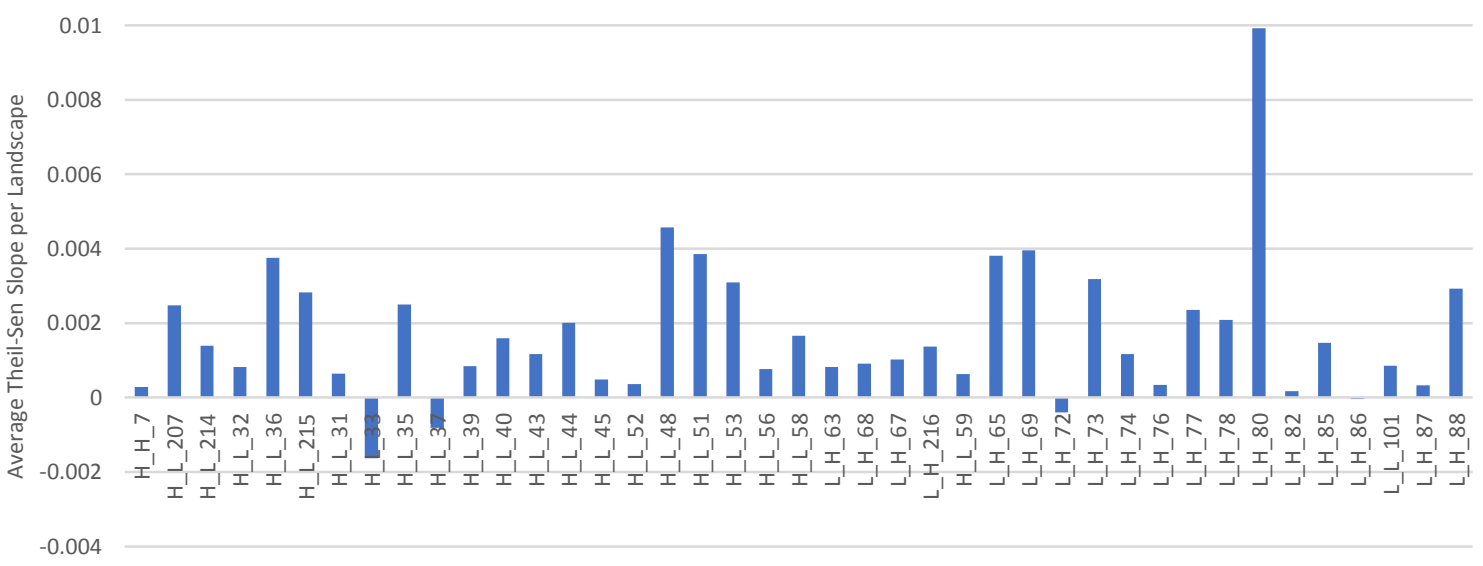

C

TS Slope for L_L Landscapes

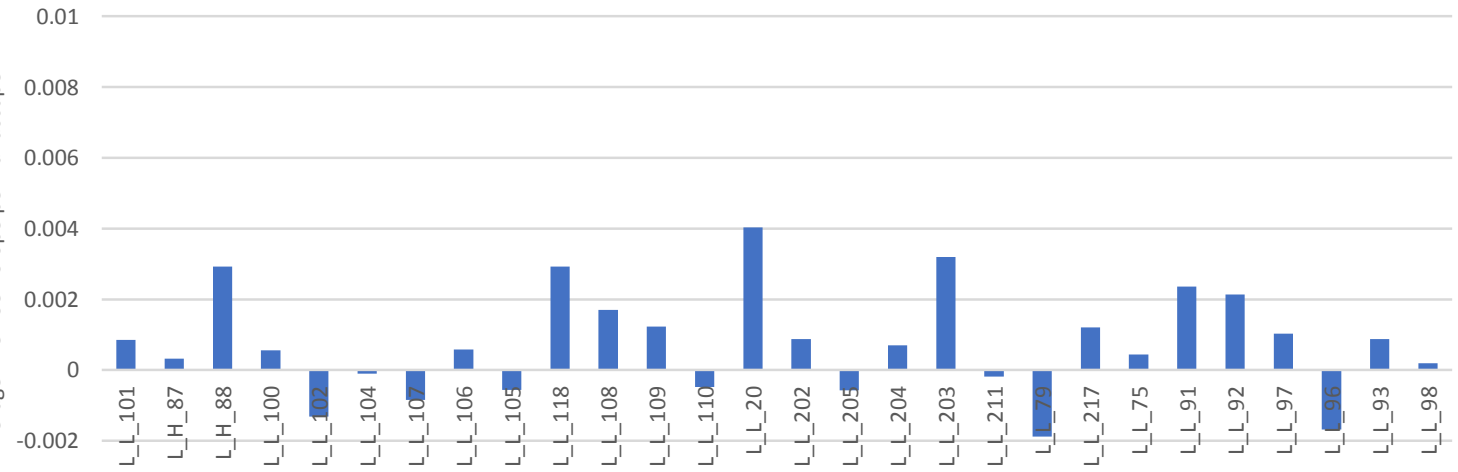

$-0.004$

Figure 6.7. Comparing MODIS NDVI average of landscape TS trend slope (irrespective of significance level) for 2000-2011 in: a) H_H, b) H_L and L_H and c) L_L sample landscapes. 


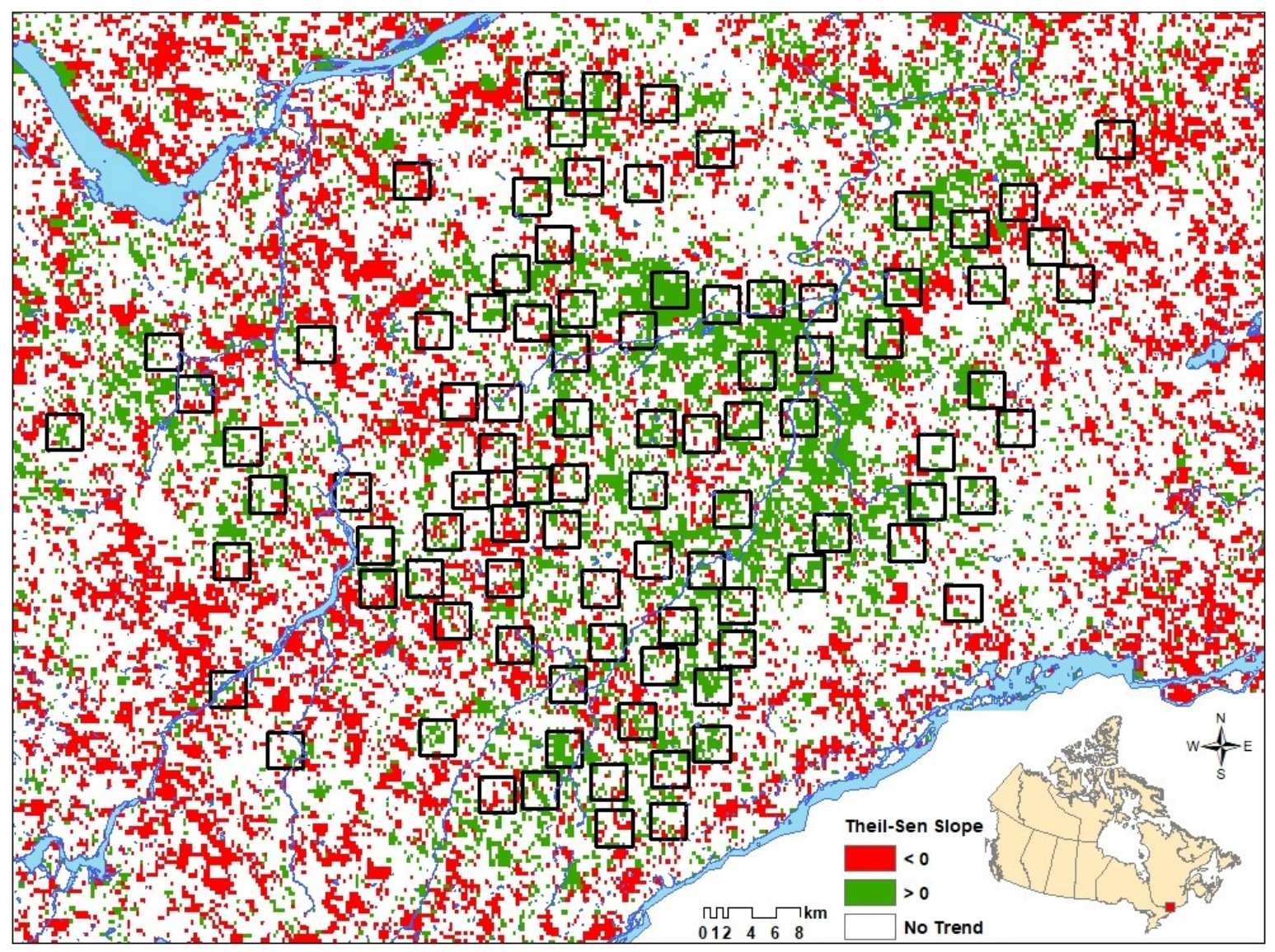

Figure 6.8. Theil-Sen slope for 2000-2011 derived from average July MODIS NDVI.

Table 6.2. The proportion of pixels in landscapes with significant positive and negative trends by landscape heterogeneity category.

\begin{tabular}{|c|c|c|c|c|c|c|}
\hline & \multicolumn{3}{|c|}{ Negative } & \multicolumn{3}{c|}{ Positive } \\
\hline & $\begin{array}{c}\text { Minimum } \\
(\%)\end{array}$ & $\begin{array}{c}\text { Maximum } \\
(\%)\end{array}$ & $\begin{array}{c}\text { Mean } \\
(\%)\end{array}$ & $\begin{array}{c}\text { Minimum } \\
(\%)\end{array}$ & $\begin{array}{c}\text { Maximum } \\
(\%)\end{array}$ & $\begin{array}{c}\text { Mean } \\
(\%)\end{array}$ \\
\hline H_H & 0 & 7.7 & 2.4 & 0 & 33.7 & 11.5 \\
\hline H_L and L_H & 0 & 22.0 & 3.6 & 1.7 & 66.0 & 13.0 \\
\hline L_L & 0 & 17.2 & 5.6 & 0 & 23.2 & 7.5 \\
\hline
\end{tabular}




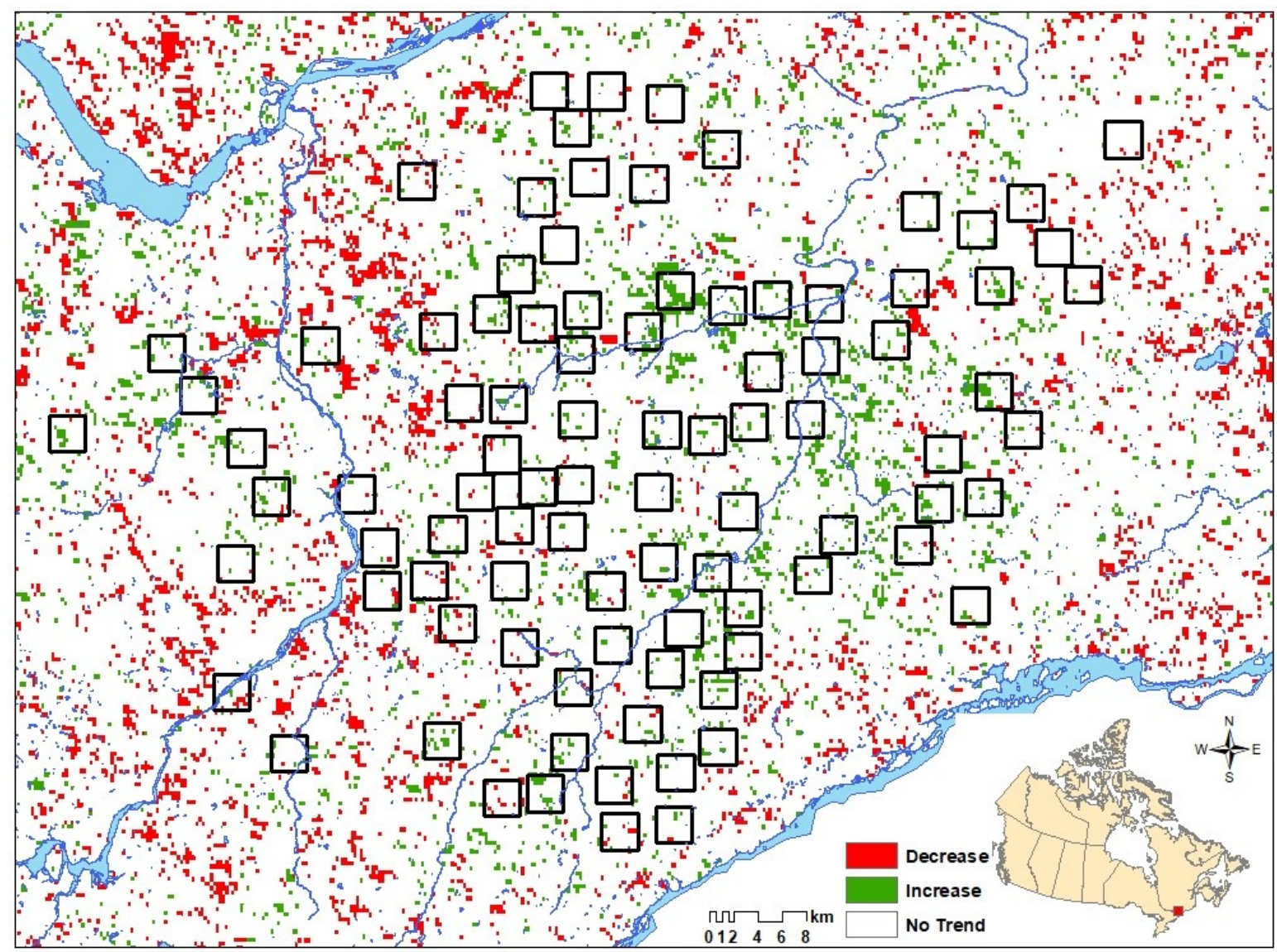

Figure 6.9. Mann-Kendall significance of TS slope for 2000-2011 derived from weekly MODIS NDVI. Red and green represent significant negative and positive trends, respectively $(\mathrm{p} \leq$ $0.05)$.
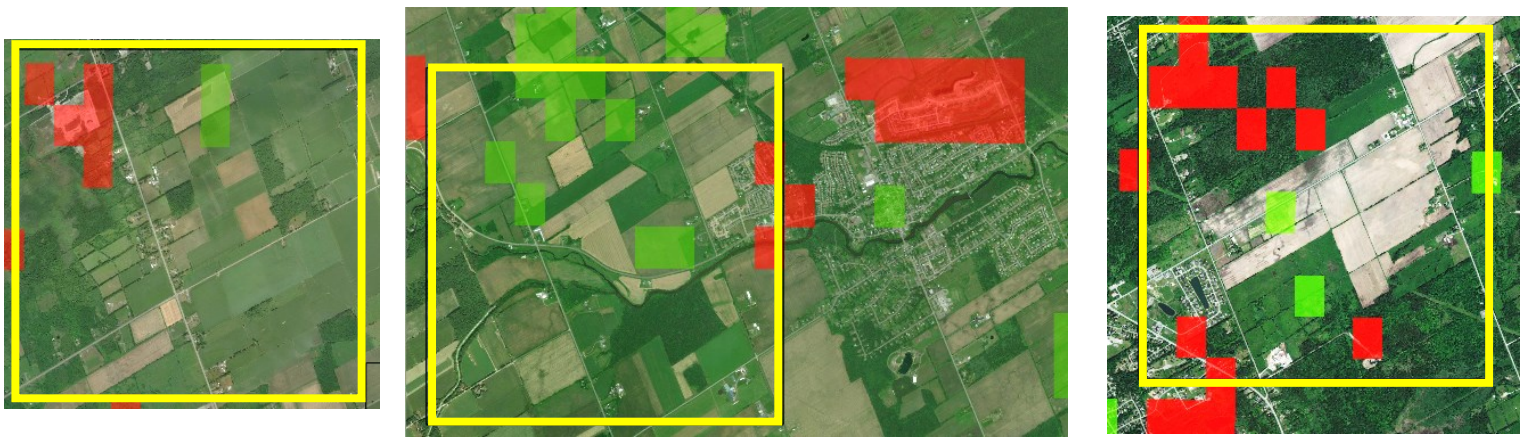

Figure 6.10. Examples of significant positive (green) and negative (red) inter-annual midsummer MODIS NDVI trends and their association with land cover. The $3 \times 3 \mathrm{~km}$ landscape bounds are shown as yellow squares. 


\subsubsection{Landsat}

\subsection{Landscape heterogeneity analysis}

As for MODIS, average mid-summer Landsat NDVI for most of the sample landscapes did not show any trends during 1985-2011. Figure 6.11 shows examples for H_H, L_L, H_L and L_H landscapes. However, 19 landscapes demonstrated a significant increase of between 0.15 and 0.2 in average NDVI (Figure 6.12 shows examples). These increases were much greater than those found with MODIS (Figure 6.6), as the time series was more than twice as long. For the period 2000-2011, Figure 6.12 shows that increases in NDVI were on the same order as those for MODIS, being about 0.03-0.04. The landscapes with significantly increasing NDVI were distributed as follows: H_H (3), H_L (3), L_H (5), and L_L (8); i.e., 16 of the 19 landscapes with significant positive trends had either low compositional and/or low configurational heterogeneity. Of these 19 landscapes and the eight MODIS landscapes with significantly increasing NDVI, four were in common: (H_H_23, H_L_53, H_L_40 and L_L_109)

Similar to MODIS, the end points of the time series did not appear to be distinctly different from data points for proximal years. To test that, the first two observations, as well as the end points of time series for those landscapes with significant increasing trend in average NDVI were removed and the $\mathrm{r}$ - and $\mathrm{p}$-values were re-calculated. In all cases, the significance level remained at $\mathrm{p} \leq$ 0.05 . 

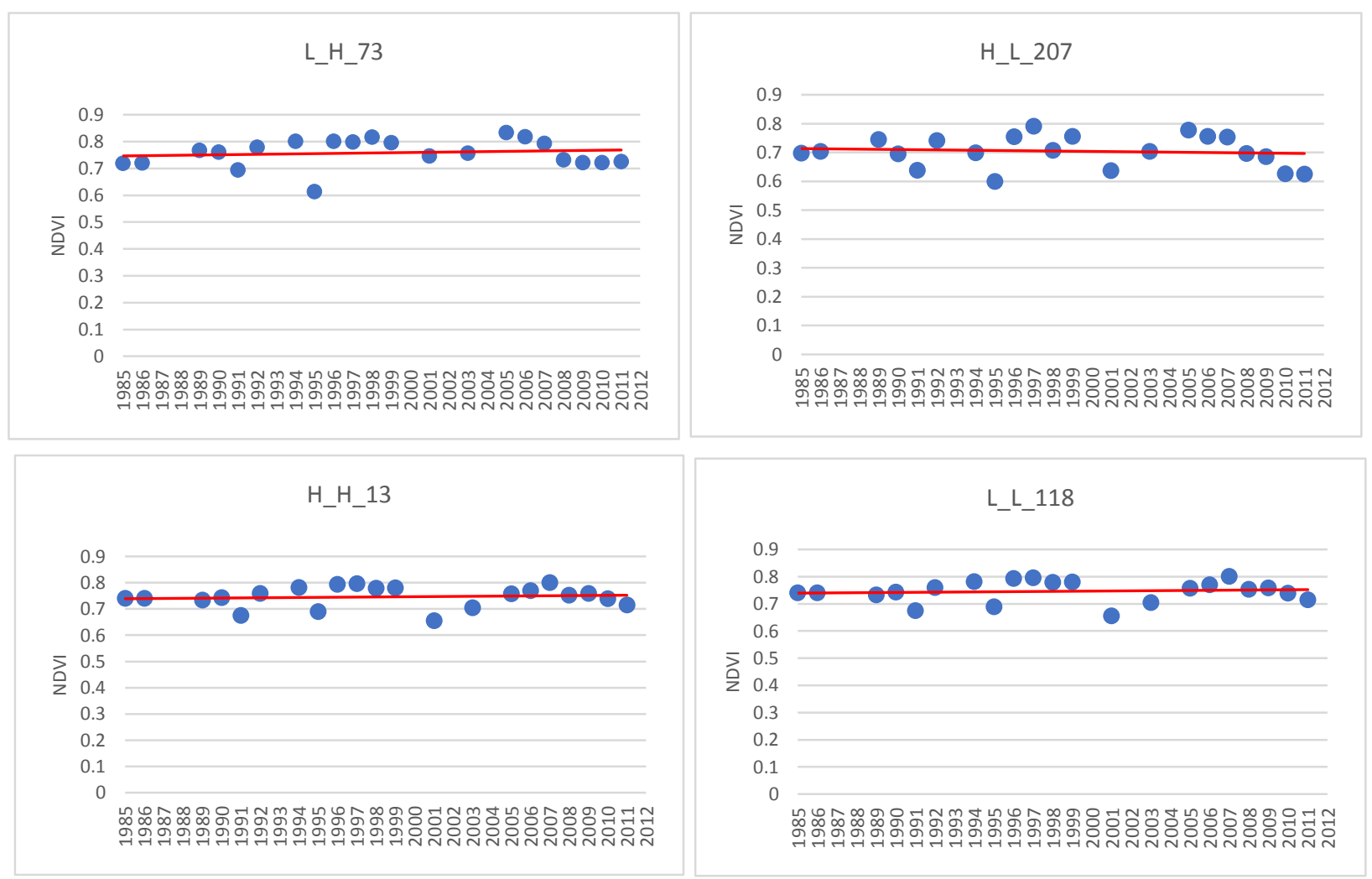

Figure 6.11. Examples of H_H, L_L, H_L and L_H sample landscapes with no significant Landsat mid-summer NDVI trend (spatial average of NDVI across each sample landscape, $\mathrm{p}>$ $0.05)$.

Analysis of the spatial average of Landsat NDVI for each sample landscape demonstrated a break in the time series and a shift in the magnitude and/or direction of the slope of the trend line in different sub-periods. This trend was especially evident between 1985-2000 and 20002011 for some sample landscapes. Of all 93 sample landscapes, 56 demonstrated a change in the direction of trend slope from positive to negative and 17 experienced a significant decrease in the slope magnitude for 2000-2011 compared to 1985-2000 (example presented in Figure 6.13). Hence, these two time periods were selected to compare the early versus late trends in NDVI at both landscape and pixel levels. In addition, as mentioned before, the 2000-2011 period was selected to match the available MODIS dataset and enable comparison between trends derived from each of them. 

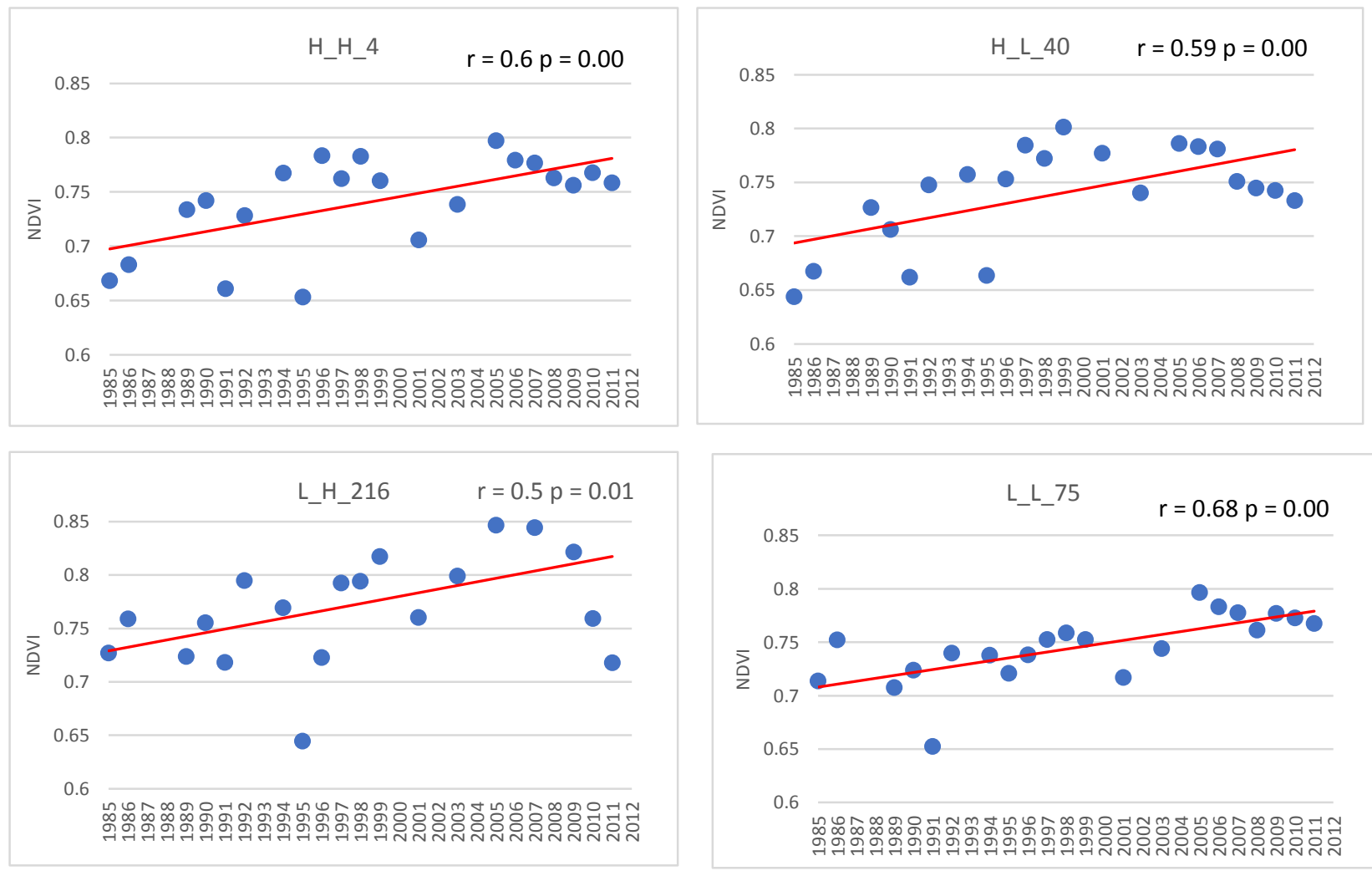

Figure 6.12. Examples of H_H, L_L, H_L and L_H sample landscapes with significant positive Landsat mid-summer NDVI trend (spatial average of NDVI across each sample landscape, $\mathrm{p} \leq$ $0.05)$.

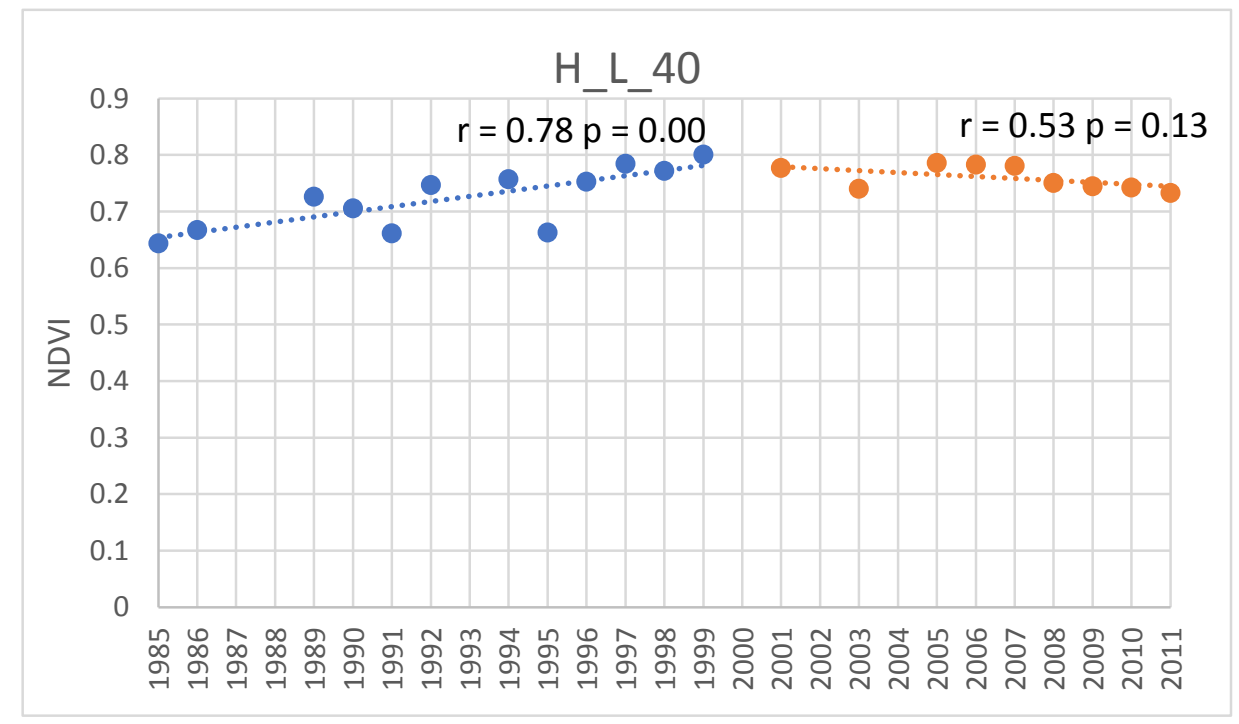

Figure 6.13. Example of temporal break in the trends of average mid-summer Landsat NDVI (for landscape H_L_40). 
Despite the very low landscape-averaged TS slopes for all datasets, the magnitude and direction of TS slopes for Landsat NDVI (without considering their significance) for the periods 1985-2000 and 2000-2011 demonstrated some patterns based on composition and configurational heterogeneity. The landscape average TS slope for 1985-2000 was positive for all landscapes. However, for the later period TS slope was often negative, and the proportions of landscapes with negative slopes was much greater for those with higher 2011-2012 heterogeneity. For H_H landscapes 26 of 28 landscapes showed negative TS slopes for 2000-2011, while for H_L and L_H landscapes 28 of 38 were negative and for L_L landscapes only 10 of 26 were negative (Figure 6.14a-c). These results indicate that all landscapes, no matter what their 2011-2012 compositional and configurational heterogeneity, demonstrated increasing NDVI in the early period. However, in the later period, higher proportions of landscapes with high compositional and/or configurational heterogeneity showed negative trends in vegetation productivity, probably as a result of removal of hedge rows and other natural vegetation. Overall, landscape mid-summer vegetation productivity had been increasing in all landscapes before about 2000, but declined after that in many landscapes that had higher 2011-12 heterogeneity. In contrast, the majority of L_L landscapes that had lower crop diversity and larger field sizes experienced an increasing trend in vegetation, probably due to more intensive cropping practices. Continued monitoring of these trends with longer time series may confirm if they are valid and statistically significant.

\subsection{Pixel Level Analysis}

In TS-CMK analysis of individual pixels within each landscape, as for the MODIS analysis, most mid-summer Landsat NDVI pixels (52.3\%) showed no significant trend (Figures 6.15 and 6.16). However, many more landscapes had larger proportions of positively trending pixels than for the MODIS analysis. 


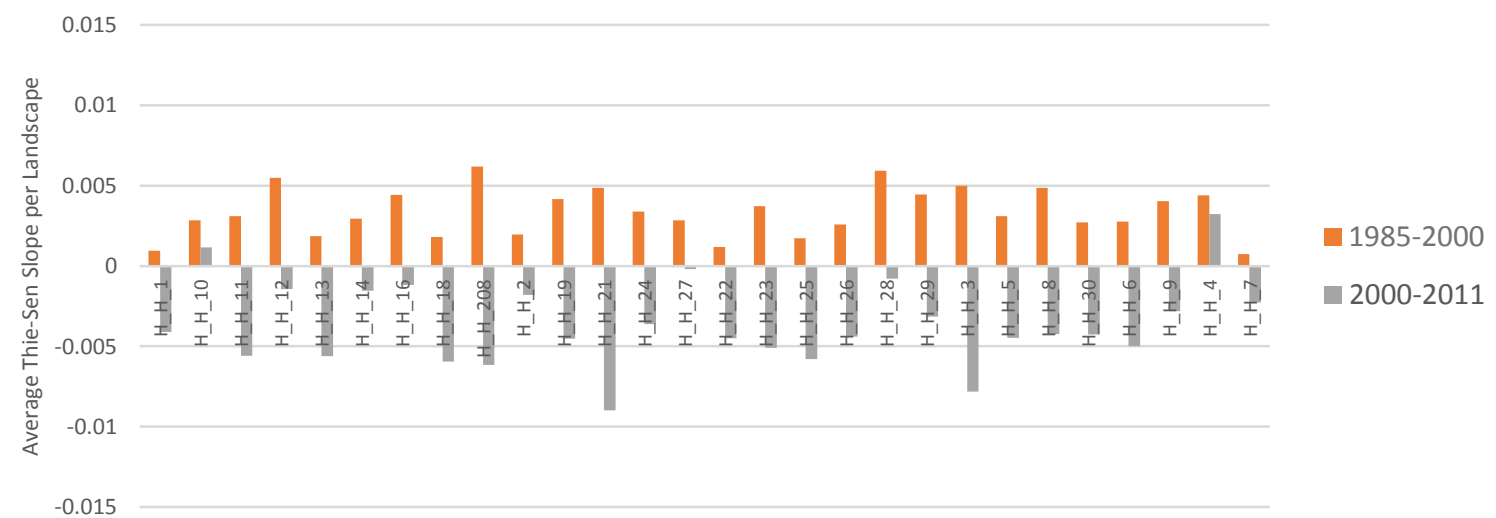

b TS slope for H_L and L_H landscapes

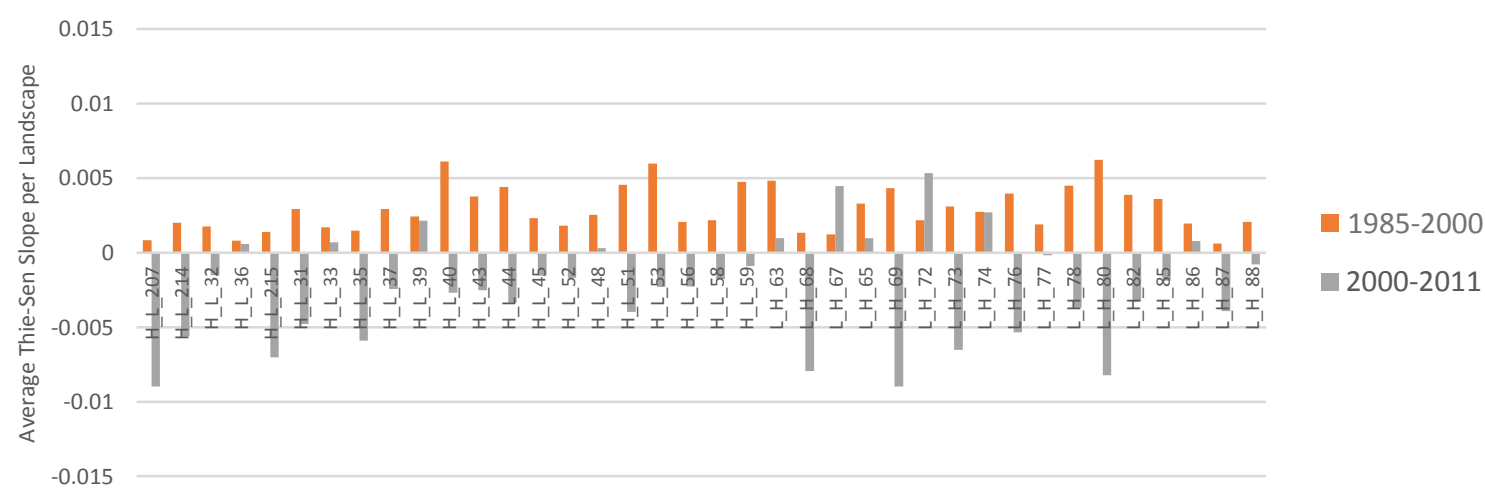

c

TS slope for L_L landscapes

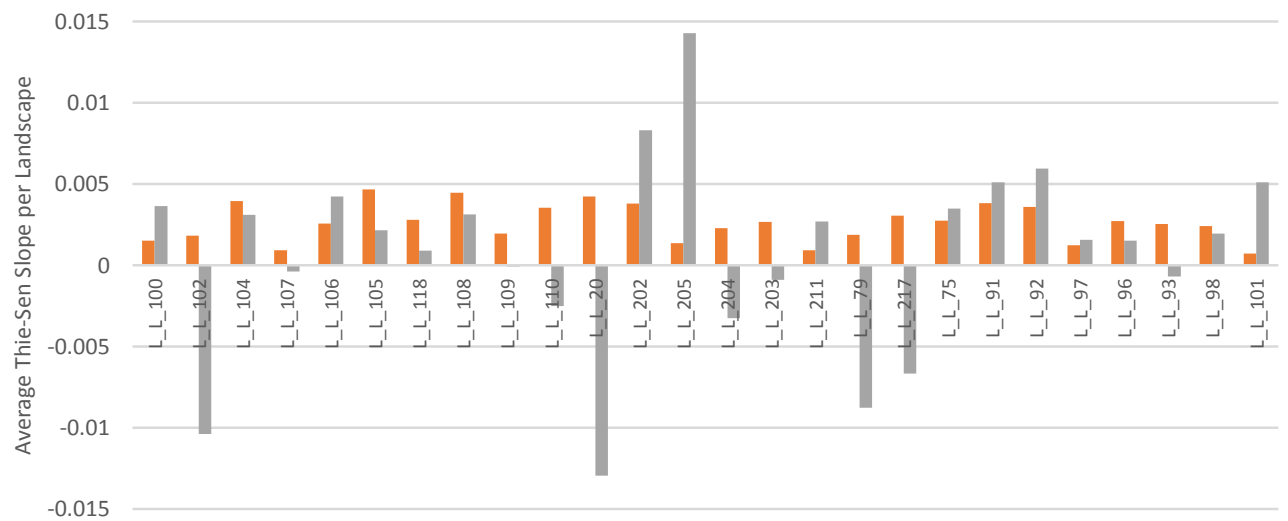

1985-2000

- 2000-2011

Figure 6.14. Comparing mid-summer Landsat NDVI TS trend slope for 1985-2000 and 20002011 in: a) H_H, b) H_L and L_H and c) L_L sample landscapes. 
The percent of pixels in each landscape with significantly positive trends was as follows: less than $10 \%$ (3 landscapes), $10-20 \%$ (25 landscapes), $20-30 \%$ (36 landscapes), and more than $30 \%$ (the remaining 29 landscapes). The highest percentage of pixels in a landscape with a significantly positive trend was $45 \%\left(\mathrm{H}_{-} \mathrm{H}_{-} 3\right)$. As for MODIS NDVI, fewer pixels showed significant negative trends. Eighty-four landscapes had less than 5\% of their pixels with significant negative trends while nine landscapes had 5-8\% negatively trending pixels.

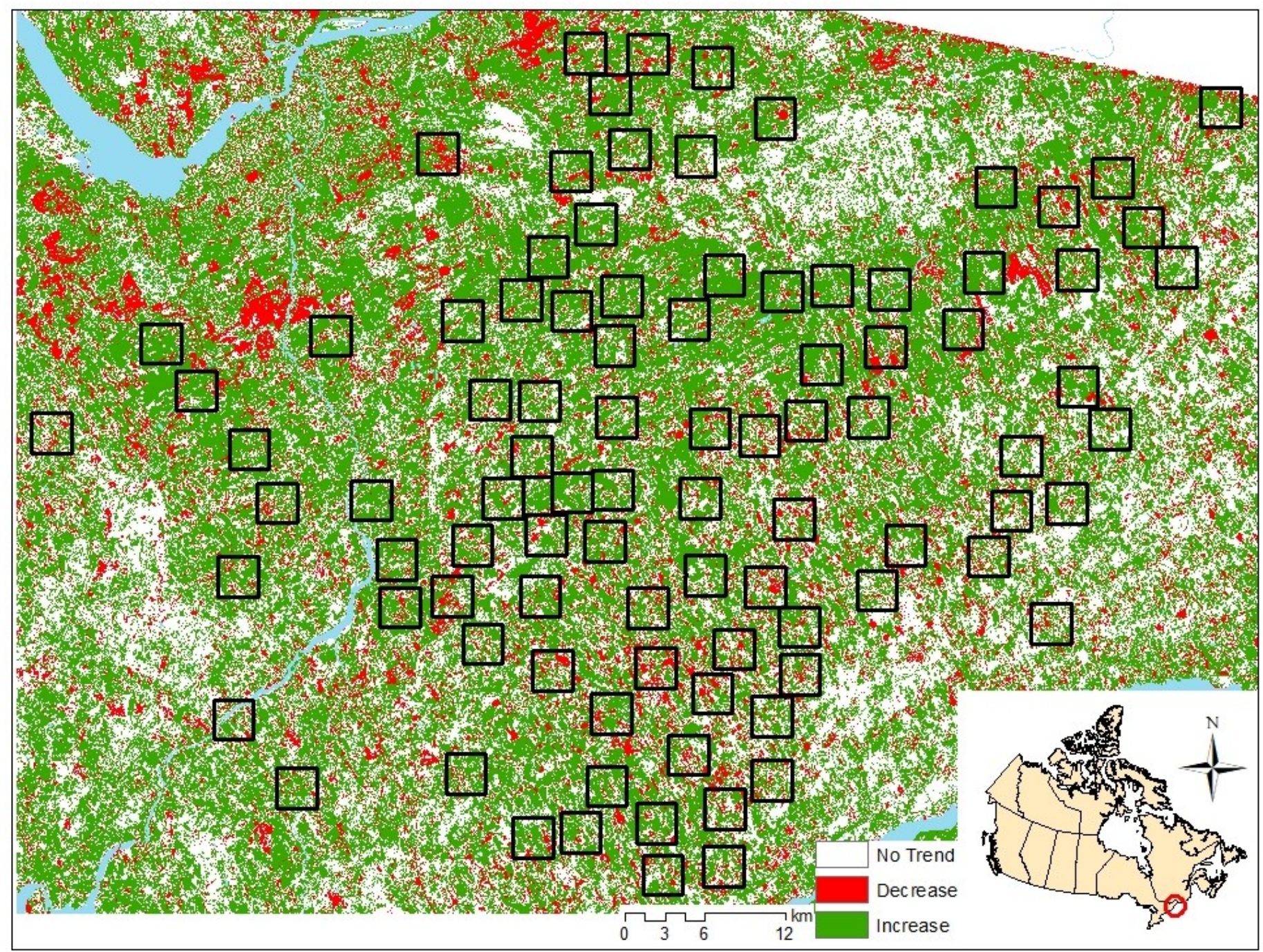

Figure 6.15. Theil-Sen slope for 1985-2011 derived from mid-summer Landsat NDVI. 


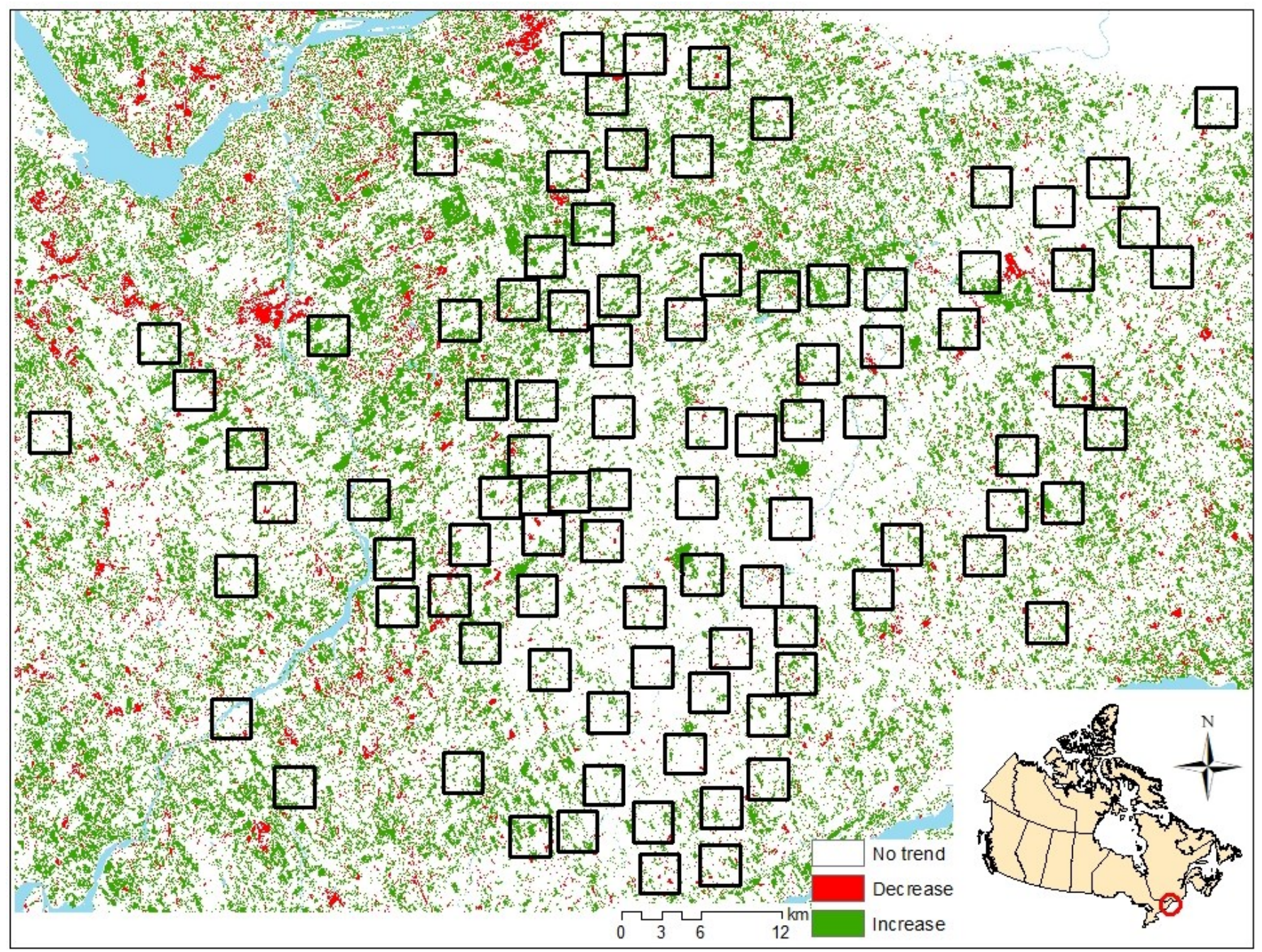

Figure 6.16. Mann-Kendall significance of slope for 1985-2011 derived from mid-summer Landsat NDVI. Red represents a significant negative trend; green represents a significant positive trend $(\mathrm{p} \leq 0.05)$.

Comparing the pixel level TS slope and CMK significance for 1985-2000 and 2000-2011 revealed that the majority of pixels in the earlier period displayed a significant positive trend while most pixels in the later period displayed no trend (Figure 6.18 and Figure 6.19). The number of pixels displaying a significant negative slope was small in both periods but greater in the later period than in the earlier period. This indicates that vegetation biomass and productivity generally increased during the earlier period and did not change or declined during the later period. 
For 1985-2000, the proportion of pixels in a given landscape showing significant positive trends were as follows: $<20 \%$ (7 landscapes); 20-29\% (26 landscapes); 30-40\% (41 landscapes); $>40 \%$ (19 landscapes). The highest percentage of positively trended pixels in a landscape was $57.5 \%$. In 2000-2011, however, only 17 landscapes had more than $10 \%$ of pixels showing a significant positive trend. The highest positive trend was $33.5 \%$. No clear pattern was found in the proportion of pixels with positive trends in 1985-2000 and the 2011-2012 landscape heterogeneity groupings. However, for 2000-2011, the top six landscapes with the highest proportion of positively trending pixels were $\mathrm{L}_{-} \mathrm{L}$. This was in agreement with the landscape level analysis, which demonstrated that $42 \%$ of landscapes with a significant increasing trend were L_L. Figure 6.17 visually compares the proportion of pixels with positive trends in each period.
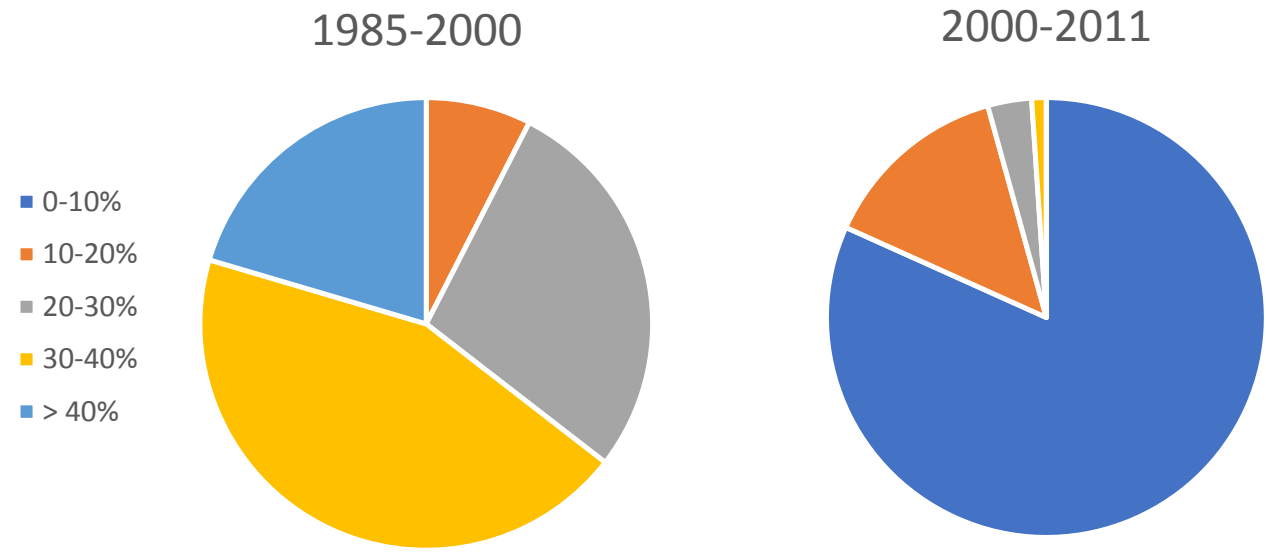

Figure 6.17. Proportion of pixels with positive mid-summer Landsat NDVI trends in all sample landscapes during 1985-2000 compared to 2000-2011.

Table 6.3 shows the proportion of positively trended pixels in sample landscapes with regards to landscape heterogeneity groupings. It indicated that the maximum, minimum and average of positively trended pixels in all landscape heterogeneity categories were higher in 1985-2000 
while the proportion of positively trended pixels decreased in 2000-2011. However, this decrease was less in L_L landscapes compared to $\mathrm{H}_{-} \mathrm{H}$ and $\mathrm{H}_{-} \mathrm{L}$ and L_H landscapes.

Table 6.3. Proportion of positively trended pixels in each landscape heterogeneity grouping.

\begin{tabular}{|c|c|c|c|c|c|c|}
\hline & \multicolumn{3}{|c|}{$1985-2000$} & \multicolumn{3}{c|}{$2000-2011$} \\
\hline & $\begin{array}{c}\text { Minimum } \\
(\%)\end{array}$ & $\begin{array}{c}\text { Maximum } \\
(\%)\end{array}$ & $\begin{array}{c}\text { Mean } \\
(\%)\end{array}$ & $\begin{array}{c}\text { Minimum } \\
(\%)\end{array}$ & $\begin{array}{c}\text { Maximum } \\
(\%)\end{array}$ & $\begin{array}{c}\text { Mean } \\
(\%)\end{array}$ \\
\hline H_H & 20 & 54.7 & 34.8 & 2.1 & 12.9 & 6.1 \\
\hline H_L and L_H & 12.7 & 57.6 & 32.5 & 1.1 & 13.2 & 5.8 \\
\hline L_L & 15.7 & 48 & 31.1 & 0.6 & 28.4 & 10.4 \\
\hline
\end{tabular}

During 1985-2000, all landscapes had less than 5\% of their pixels with a significant negative trend. In 2000-2011, 26 sample landscapes had less than 1\% negative pixels, 11 of which were L_L. Four landscapes had more than 5\% negatively trended pixels with the highest proportion in a landscape being 9.1\% (L_L_102). The proportion of negatively trended pixels in sample landscapes with regards to landscape heterogeneity groupings is presented in Table 6.3. For H_H, HL and L_H landscapes, a higher proportion of negatively trended pixels was observed in 20002011 compared to 1985-2000. In contrast, for L_L landscapes a slight decrease in the percentage of negatively trended pixels was observed 2000-2011 compared to 1985-2000 and the average was lowest compared to other heterogeneity groupings.

Table 6.4. Proportion of negatively trended pixels in each landscape heterogeneity grouping.

\begin{tabular}{|c|c|c|c|c|c|c|}
\hline & \multicolumn{3}{|c|}{$1985-2000$} & \multicolumn{3}{c|}{$2000-2011$} \\
\hline & $\begin{array}{c}\text { Minimum } \\
(\%)\end{array}$ & $\begin{array}{c}\text { Maximum } \\
(\%)\end{array}$ & $\begin{array}{c}\text { Mean } \\
(\%)\end{array}$ & $\begin{array}{c}\text { Minimum } \\
(\%)\end{array}$ & $\begin{array}{c}\text { Maximum } \\
(\%)\end{array}$ & $\begin{array}{c}\text { Mean } \\
(\%)\end{array}$ \\
\hline H_H & 0.2 & 3.7 & 1.4 & 0.5 & 7 & 1.9 \\
\hline H_L and L_H & 0.5 & 5.2 & 1.7 & 0.2 & 5.8 & 2.2 \\
\hline L_L & 0.4 & 4.8 & 2.0 & 0.1 & 9.1 & 1.7 \\
\hline
\end{tabular}

The percentage of pixels with no significant trend was $41.2 \%$ and $71.8 \%$ for all landscapes in 1985-2000 and 2000-2011, respectively. 
The above analysis indicates that H_H landscapes had higher proportions of pixels with significant positive trends and lower proportions of pixels with significant negative trends in both periods compared to H_L, L_H and L_L landscapes. This shows that productivity increases were more widespread across landscapes with more crop types (compositional) and smaller field size (configurational), particularly during the $1985-2000$ period.

The proportion of positive and negative pixels with regard to the landscape heterogeneity groupings was not in agreement between MODIS and Landsat for 2000-2011, since MODIS demonstrated a higher proportion of positive trending pixels in $\mathrm{H}_{-} \mathrm{H}, \mathrm{H} \_\mathrm{L}$ and $\mathrm{L}_{-} \mathrm{H}$ landscapes and higher proportion of negative trending pixels in L_L landscapes while the opposite was the case for Landsat. This could be due to more spatial detail provided by Landsat's higher spatial resolution, while the large pixels MODIS generalized changes in many areas.

Investigation of the spatial distribution of the positive and negative TS slopes showed that, as for MODIS, these trends were mostly due to inter-annual variability in vegetation productivity in the cropped portion of the landscapes and were associated with increasing crop productivity. While most of the natural and semi-natural areas generally demonstrated no trends during both periods, many significant negative trends within the sample landscapes, especially in the later period, were deemed to be associated with land conversion and destruction of natural and seminatural lands especially wooded patches (Figure 6.20). In addition, some individual fields also demonstrated significant negative trends (Figure 6.21). The higher spatial resolution of Landsat enabled detection of the spatial distribution of significant trends at a much higher detail. For instance, examples of significant negative trends were evident at field edges, demonstrating the destruction of linear woody features and hedgerows (Figure 6.22). 


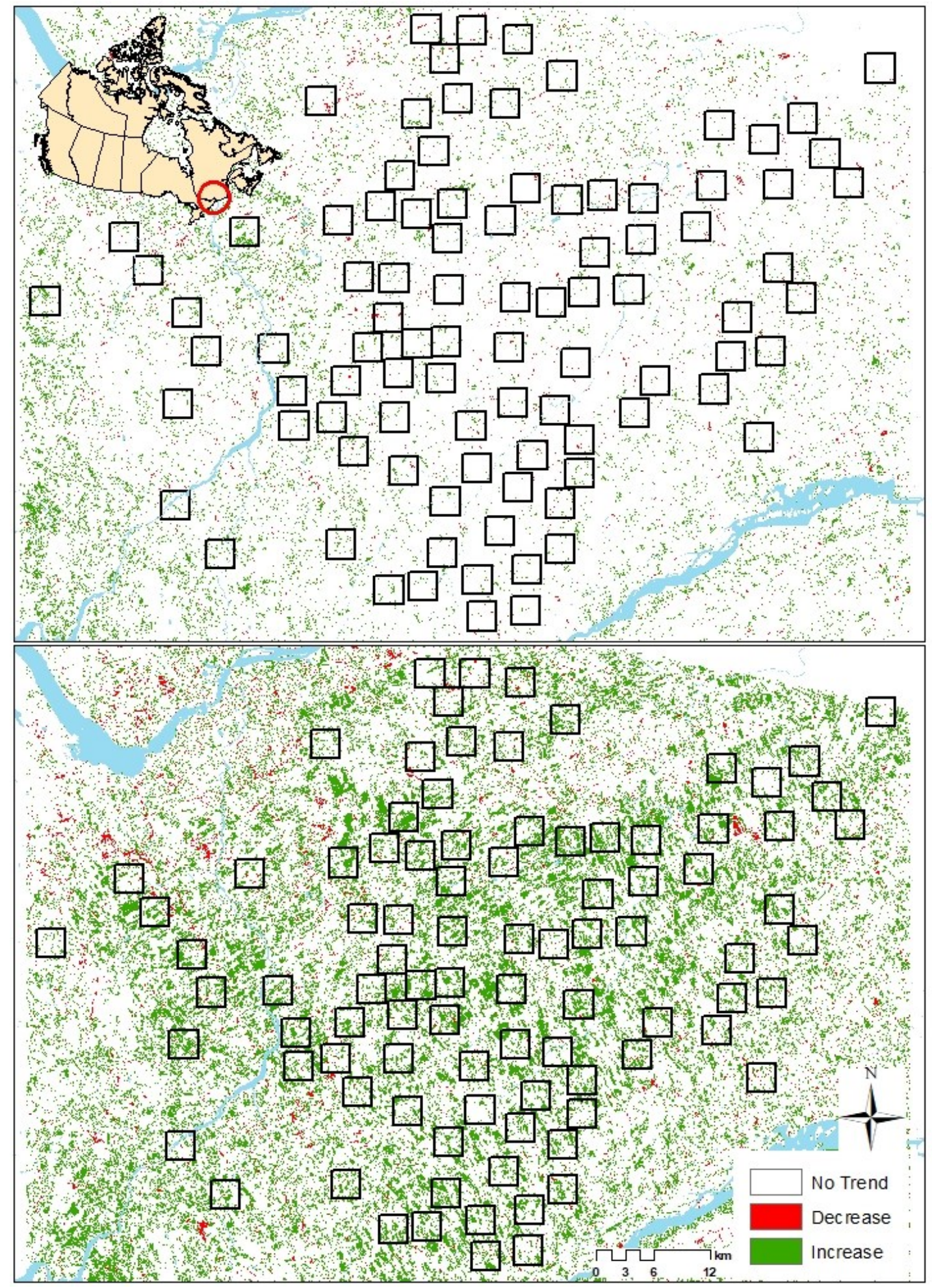

Figure 6.18. Direction of significant $(\mathrm{p} \leq 0.05)$ Mann-Kendall) trend slopes for mid-summer Landsat NDVI: 1985-2000 (bottom) and 2000-2011 (top). 

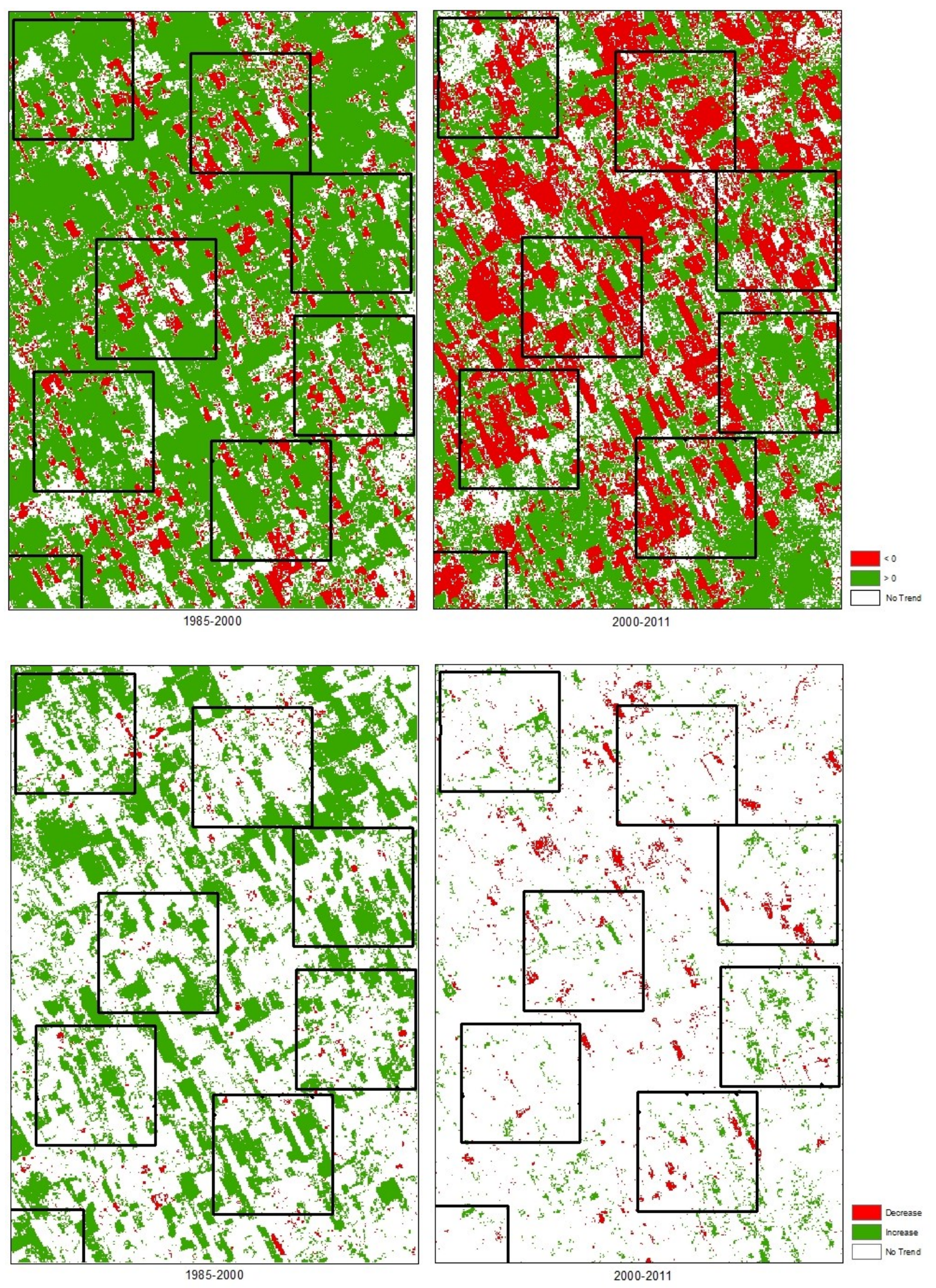

Figure 6.19. Comparing mid-summer Landsat NDVI trends for 1985-2000 and 2000-2011 in example landscapes. (top) Theil-Sen slope for all pixels. Red represents negative slope and green represents positive slope, and (bottom) Mann-Kendall significance. Red represents a significant negative trend and green represents a significant positive trend $(\mathrm{P} \leq 0.05)$. 

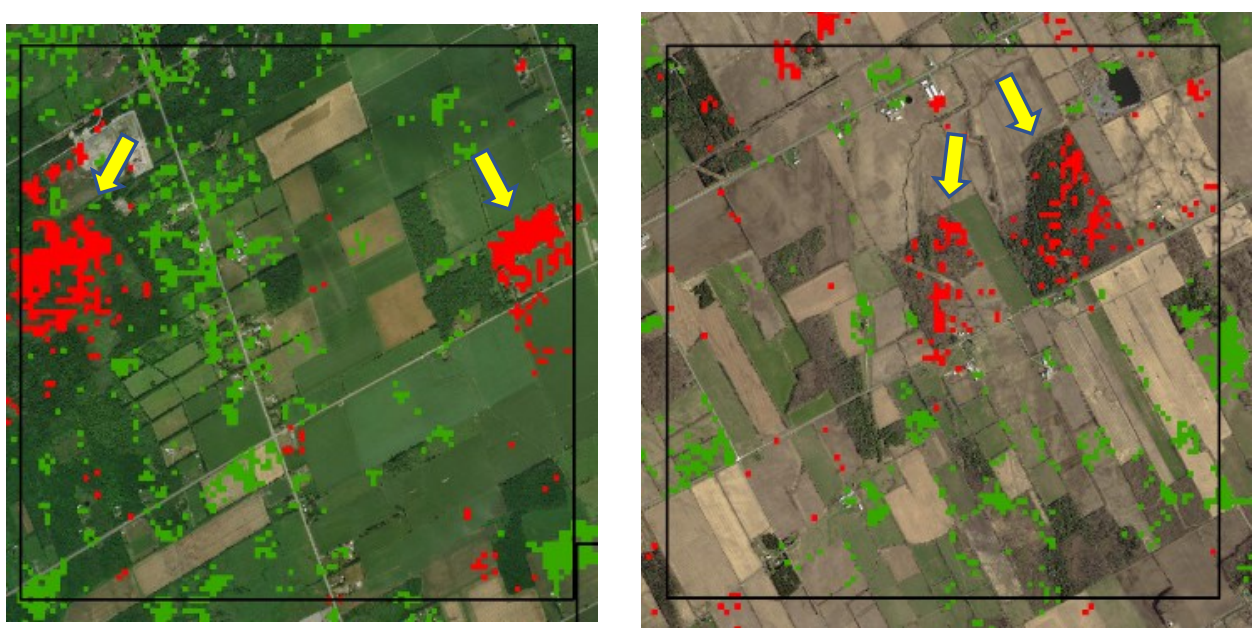

Figure 6.20. Examples of CMK significant negative trends in mid-summer Landsat NDVI for 2000-2011 associated with natural and semi-natural patches (yellow arrows).
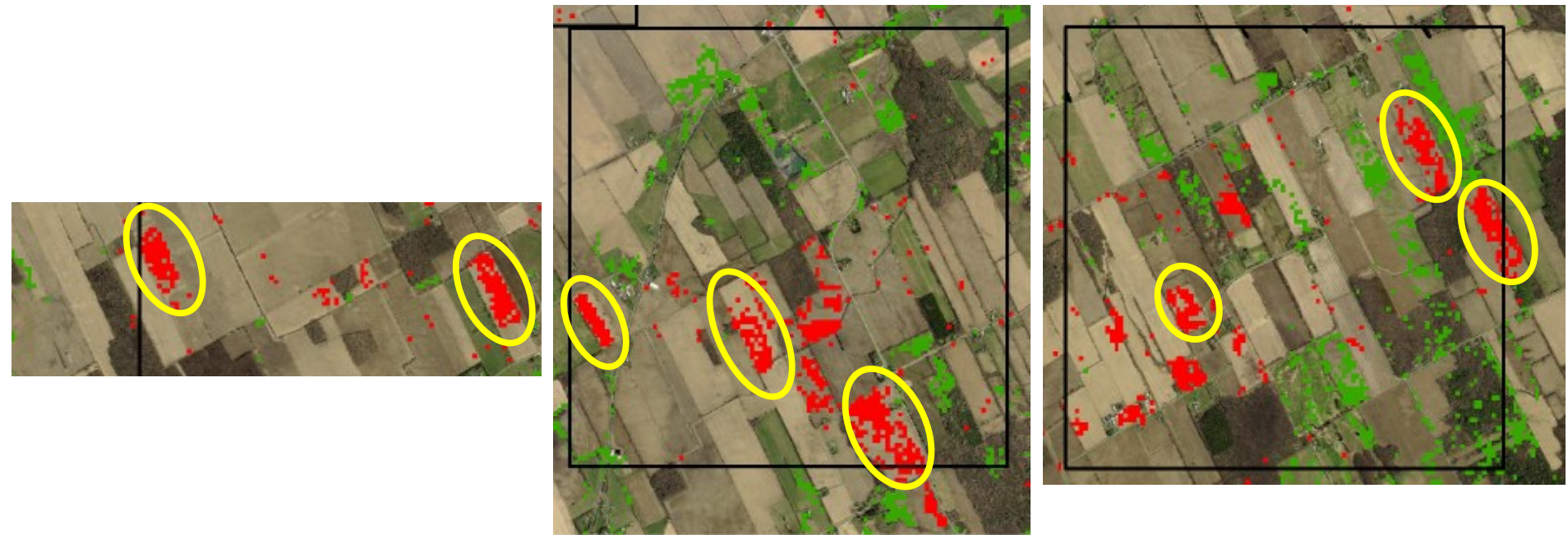

Figure 6.21. Examples of CMK significant negative trends in mid-summer Landsat NDVI for 2000-2011 associated with individual fields (yellow circles).
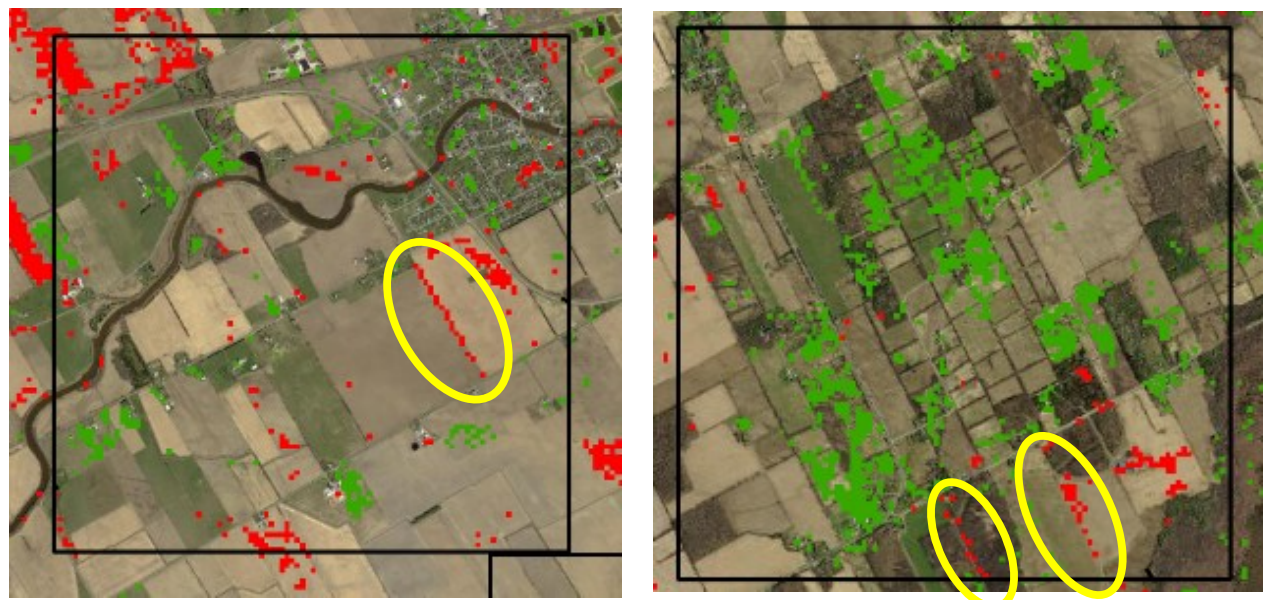

Figure 6.22. Examples of CMK significant negative trends in mid-summer Landsat NDVI for 2000-2011 associated with individual field edges (yellow circles). 


\subsubsection{Comparing MODIS and Landsat NDVI trend analyses}

In order to evaluate the effect of spatial resolution in portraying temporal trends, the average of MODIS weekly composite NDVI time series in July were compared to single date mid-summer Landsat NDVI time series for 2000-2011.

The output of MODIS NDVI trend analysis (map of pixels with significant positive and negative trends) was resampled to match the $30 \mathrm{~m}$ Landsat pixels and the maps were then crosstabulated to calculate the numbers of coincident pixels of both datasets that demonstrated significant negative or positive trends, or no significant trend. While $61.5 \%$ of all pixels for MODIS and $70.8 \%$ of all pixels for Landsat demonstrated no significant trend, the cross tabulation matrix (Table 6.4) demonstrated that $92 \%$ of MODIS pixels with no trend were in agreement with those of Landsat and $88.4 \%$ of Landsat pixels with no trend were in agreement with those of MODIS. However, only $3.1 \%$ of MODIS significant negative pixels and $6.3 \%$ of MODIS significant positive pixels were in agreement with the coincident Landsat pixels, while $6.3 \%$ of Landsat significant negative pixels and $8 \%$ of Landsat significant positive pixels were in agreement with the coincident MODIS pixels. While MODIS and Landsat did not concur well with regards to the proportion of positively and negatively trended pixels at the individual pixel level, the general spatial distribution of these pixels coincided well in many areas. Landsat generally identified more pixels with significant (positive or negative) trends and provided more spatial detail. Figures 6.23 and Figure 6.24 show examples of areas with spatially compatible significant trends in both datasets as circles, while areas with significant trends identified by only one dataset are indicated by arrows. In addition, these figures suggest that Landsat pixels with significant trends are more spatially precise since they mostly fell within the boundaries of a given field, while MODIS pixels fell outside the boundaries of fields in areas where change had not occurred. 
Table 6.5. Cross-tabulation of numbers of pixels identified as having significant trends or no trend in MODIS and Landsat mid-summer NDVI time series.

\begin{tabular}{|r|c|r|r|r|r|r|}
\hline \multicolumn{2}{|c|}{} & \multicolumn{6}{|c|}{ MODIS } \\
\cline { 3 - 7 } \multicolumn{2}{|c|}{} & Negative & Positive & No trend & \multicolumn{1}{c|}{ Sum } & \% Agreement \\
\hline \multirow{4}{*}{ Landsat } & Negative & $\mathbf{1 1 9 0}$ & 2300 & 34346 & 37836 & 3.1 \\
\cline { 2 - 7 } & Positive & 1440 & $\mathbf{4 4 4 5}$ & 64402 & 70287 & 6.3 \\
\cline { 2 - 7 } & No trend & 16294 & 48537 & $\mathbf{7 4 9 8 4 9}$ & 814680 & 92.0 \\
\cline { 2 - 7 } & Sum & 18924 & 55282 & 848597 & $\mathbf{9 2 2 8 0 3}$ & \\
\cline { 2 - 7 } & \% Agreement & 6.3 & 8.0 & 88.4 & & \\
\hline
\end{tabular}
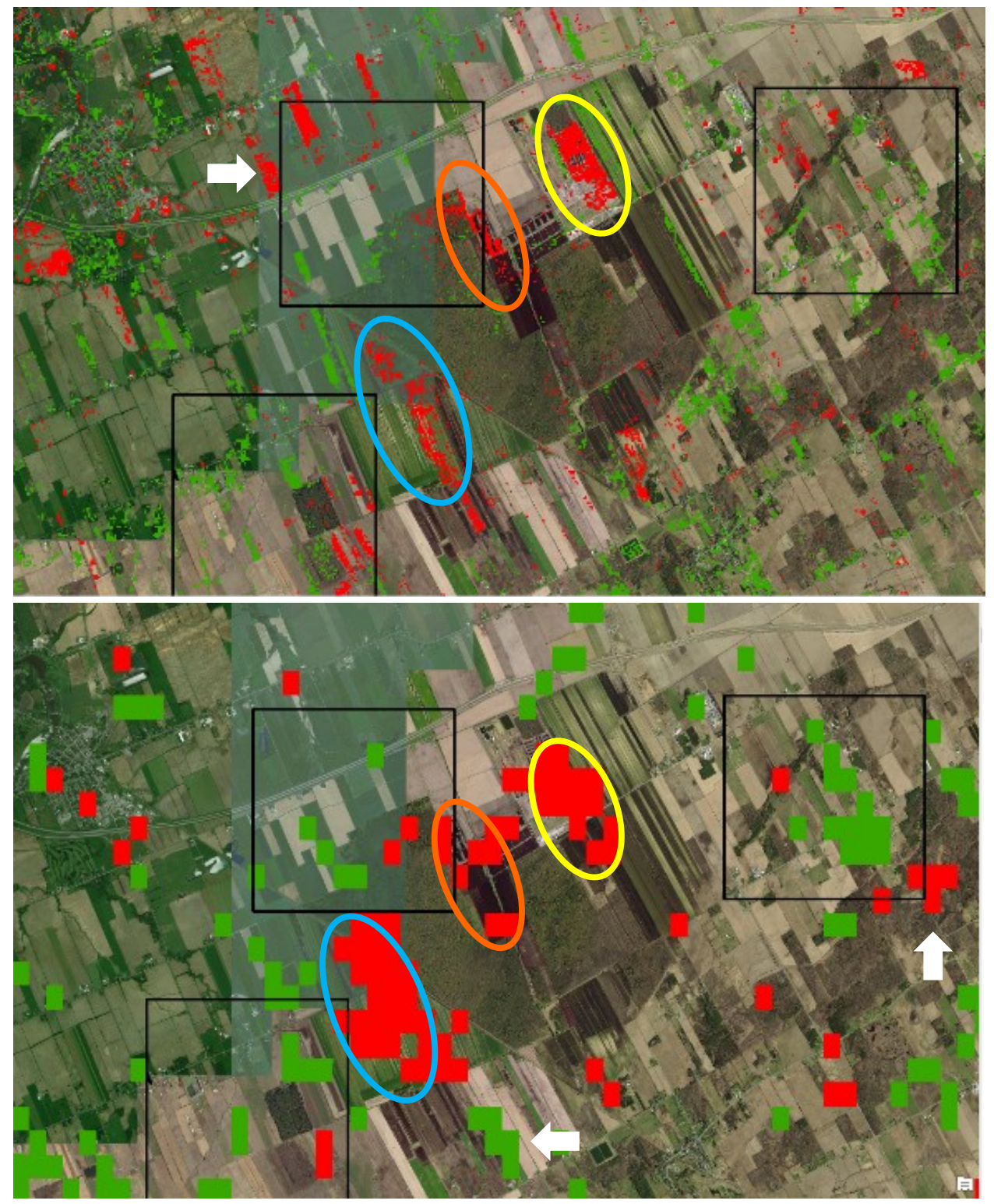

Figure 6.23. Comparing Landsat (top) and MODIS (bottom) spatial patterns of significant trends in mid-summer NDVI. Red = negative and green $=$ positive trends. Ellipses with the same colours show the same location on both maps with spatially overlapping significant trends. Arrows show instances of significant positive or negative trends identified by only one dataset. 

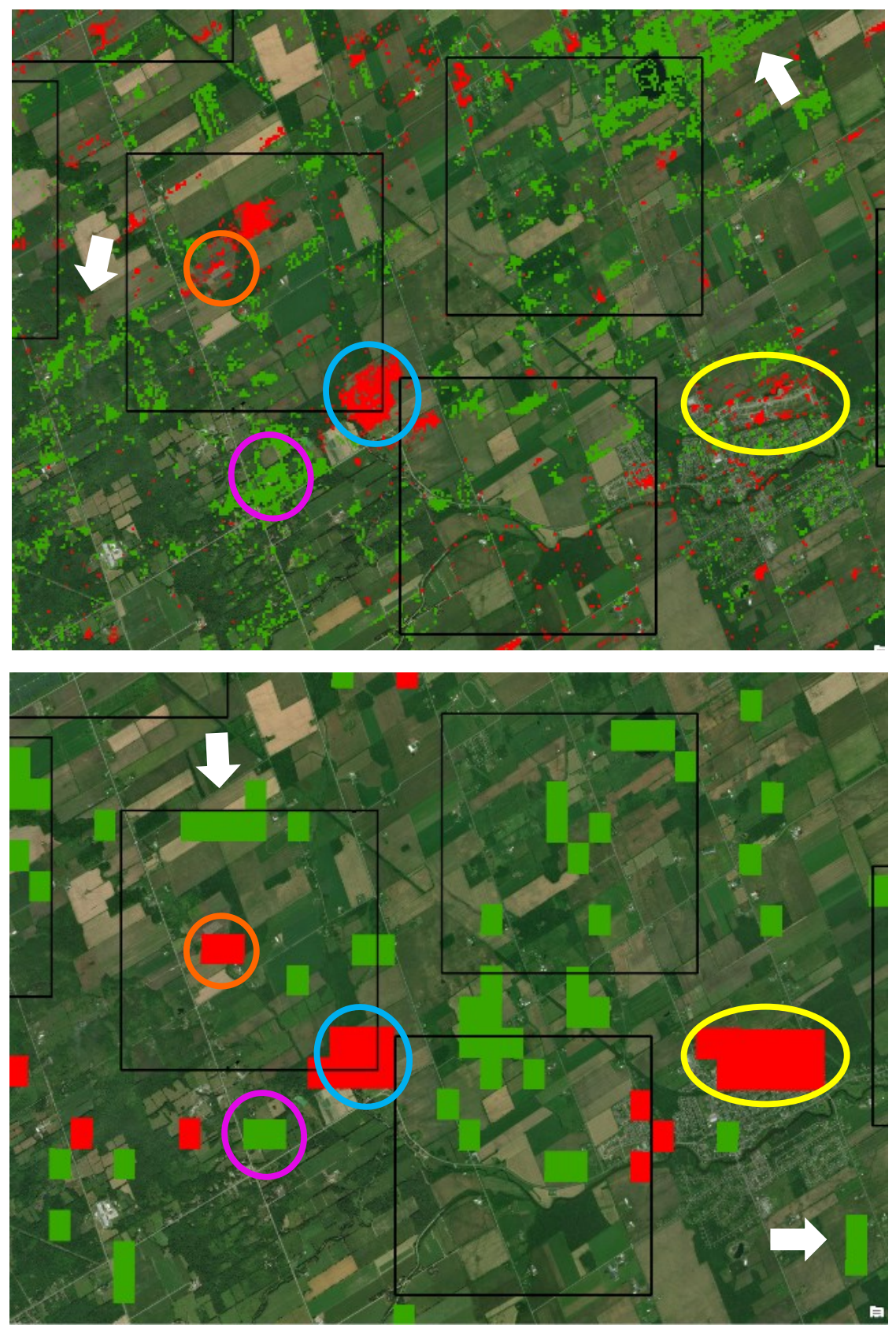

Figure 6. 24. Comparing Landsat (top) and MODIS (bottom) patterns of CMK significant trends in mid-summer NDVI and examples of the spatial distribution of significant negative and positive pixels in part of the study area. Red = negative and green = positive trends. Ellipses/circles with the same colours show locations with spatially overlapping significant trends. Arrows show instances of significant positive or negative trends identified by only one dataset. 


\subsubsection{Modelling biodiversity and inter-annual landscape dynamics}

\subsubsection{Relationships between biodiversity and the proportion of landscape pixels with positive and negative TS slope}

The relationships between the percentage of all pixels in a given landscape with a negative or positive TS slope (without regard for slope significance) or no TS slope (TS slope $=0.00$ ) and 2011-2012 plant, beetle, bird and butterfly gamma diversity were tested independently. Explanatory variables were extracted from the July average of MODIS NDVI and Mean EOS NDVI (the variable that showed the strongest temporal trend) for the period 2000-2011 and from mid-summer Landsat NDVI for the periods 1985-2011, 1985-2000, and 2000-2011. No significant relationships were found for MODIS NDVI and Mean EOS NDVI. For Landsat, 20112012 plant diversity was negatively related to the percentage of 2000-2011 negative NDVI TS slopes $(r=0.49, p=0.00$ and positively related to the percentage of 2000-2011 positive NDVI TS slopes $(\mathrm{r}=0.36, \mathrm{p}=0.00)$. For Landsat in the earlier period $(1985-2000), 2011-2012$ plant diversity was positively related to the percentage of pixels with no trend $(r=0.35, p=0.00)$. These results are presented in Figure 6.25.
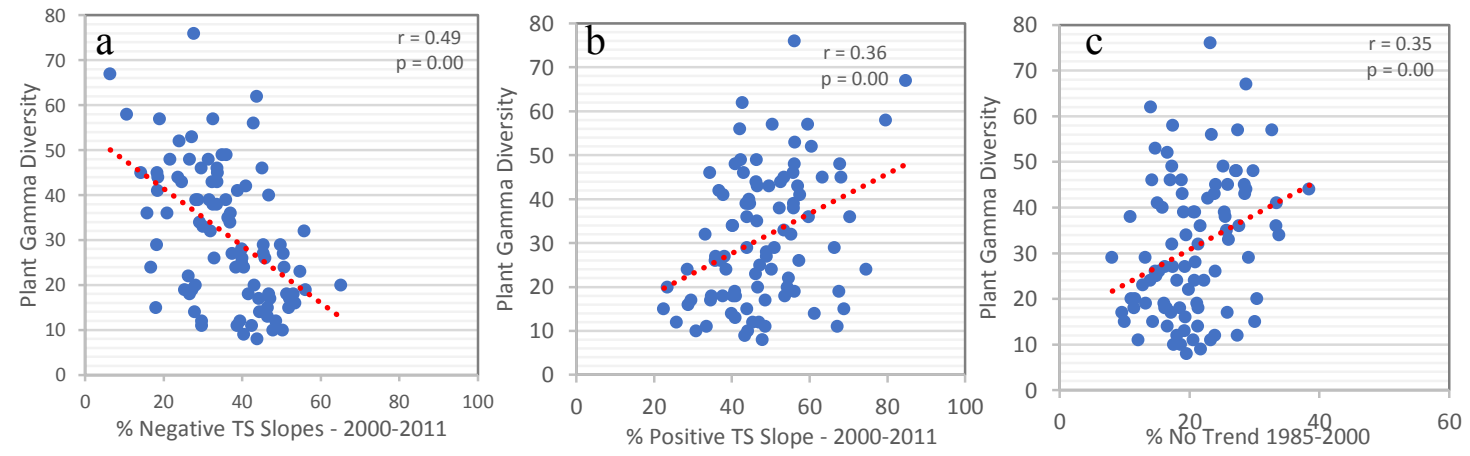

Figure 6.25. The relationships between plant gamma diversity and the percentage of pixels in each landscape with negative (a) and positive (b) TS slopes as well as the percentage of pixels with no trend (TS slope $=0.00)(\mathrm{c})$ for given time periods. TS slopes were derived from Landsat mid-summer NDVI time series. 
Beetle gamma diversity showed a positive relationship with the percentage of pixels with no TS trends in the later period $(r=0.38, p=0.00$; Figure 6.26). No other significant relationships were found for beetles, butterflies and birds.

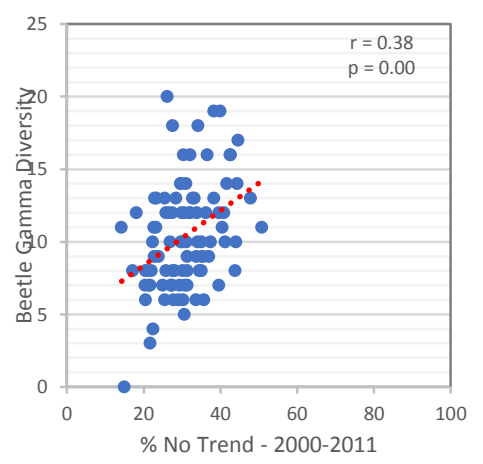

Figure 6.26. The relation between beetle gamma diversity and the percentage of pixels with no TS slope $($ TS slope $=0.00)$ in the period 2000-2011. TS slopes were derived from Landsat NDVI time series.

Similar tests to those above were conducted using the percentage of pixels with CMK significant negative and positive trends, as well as no trend. Again, MODIS NDVI did not produce any significant relationships. For Landsat, the only significant relationships were between 20002011 trends and plant diversity of 2011-2012, which was positively related to the percentage of pixels with a significant positive trend and no trend $(\mathrm{r}=0.44 \mathrm{p}=0.00$ and $\mathrm{r}=0.34 \mathrm{p}=0.00$, respectively; Figure 6.27). In Figure 6.27, three landscapes are distinctly separate from the other data points at the upper end of the models, having more than $20 \%$ of their pixels with significant positive NDVI trends (left graph) and less than $80 \%$ of their pixels showing no trend for 20002011 (right graph). These landscapes were L_L_75, one of 19 sample landscapes with a significant increasing trend at the landscape level, and L_L_202 and L_L_205, both showing no significant trend. The effect of these three sample landscapes on the relationship with plant gamma diversity was tested by removing them from the data set and recalculating the correlation. The new bi-variate models had lower $\mathrm{r}$-values but were still significant (for $\%$ positive trends $\mathrm{r}=$ 
$0.33, \mathrm{p}=0.00$ and for $\%$ no trend $\mathrm{r}=0.34, \mathrm{p}=0.00$ ), hence these sample landscapes were not deemed to affect the relationship of $\%$ positive trends and $\%$ no trends and were retained in the models.

Although no evidence of a lag effect was found in the relationships of pre-2011 Landsat variables and 2011-2012 wild plant diversity (Chapter 5), the inter-annual variability of landscapes at the pixel level may indicate evidence of a time lagged response. Given that most of the significant positive trends (i.e. significant increases in vegetation productivity) occurred in the earlier period and far fewer instances of pixels with significant positive trends occurred in the later period, the significant relationship of wild plant diversity with the proportion of pixels with significant positive trends might be an indicator of time lagged response of plant diversity to an increase in landscape productivity.
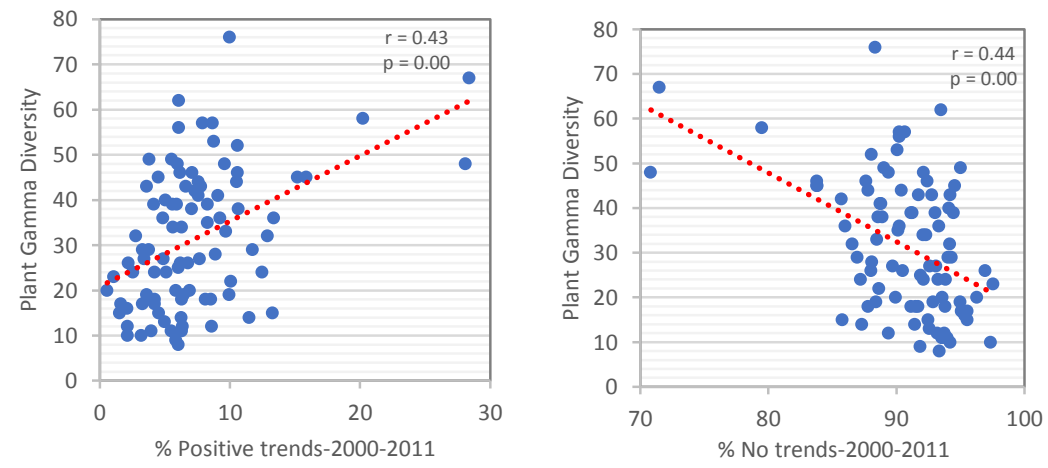

Figure 6.27. The relation between plant gamma diversity and the percentage of CMK significant positive Landsat inter-annual NDVI pixels and no trends in the period 2000-2011.

\subsubsection{Relationships between biodiversity and landscape vegetation dynamics}

The inter-annual variability of NDVI at the landscape level (measured by the coefficient of variation $(\mathrm{CV})$ ) was calculated for MODIS and Landsat time series as the temporal $\mathrm{CV}$ of the spatial mean of NDVI and the temporal CV of spatial CV of NDVI, and the relationship between these measures and biodiversity was tested. No relationship was found between biodiversity and 
MODIS-derived temporal CV of landscape Mean and spatial CV of NDVI. Significant negative relationships were found between 2011-2012 plant and beetle gamma diversity and Landsatderived temporal CV of mean landscape NDVI for 1985-2011 $(\mathrm{r}=0.52$ and $\mathrm{r}=0.45$, respectively; $\mathrm{p}=0.00$ for both; Figure 6.28). Plant and beetle gamma diversity also demonstrated a significant negative relationship with Landsat-derived temporal CV of mean landscape NDVI for 1985-2000 $(r=0.47$ and $r=0.30$, respectively; $p=0.00$ for both; Figure 6.29) and for 2000-2011 $(r=0.34$ and 0.35 , respectively and $p=0.00$ for both; Figure 6.30 ). This indicated that the variability in average landscape productivity through time is negatively related to plant and beetle diversity and landscapes with less temporal variability supported more wild plants and beetles species. This supports the result of the previous landscape level trend analysis of this chapter, where both plants and beetles demonstrated significant positive trends with the proportion of pixels with no trends. In addition, plants demonstrated a significant negative relation with NDVI spatial CV (Chapter 4) and in Chapter 5 both beetles and plants demonstrated strong negative relations with NDVI spatial CV. Combined, these results indicate that more spatially and temporally stable landscapes (e.g. with fewer disturbances, interventions, or crop changes over space and time) can support more beetle and plant species. The relationships for both plants and beetles were stronger in 19852011 compared to the early and late periods independently. This could be due to the longer period and more sample points compared to the sub-periods. No significant relationships between gamma diversity and the temporal CV of landscape CV for MODIS-derived or Landsat-derived NDVI were noted $(\mathrm{p}>0.05)$. 

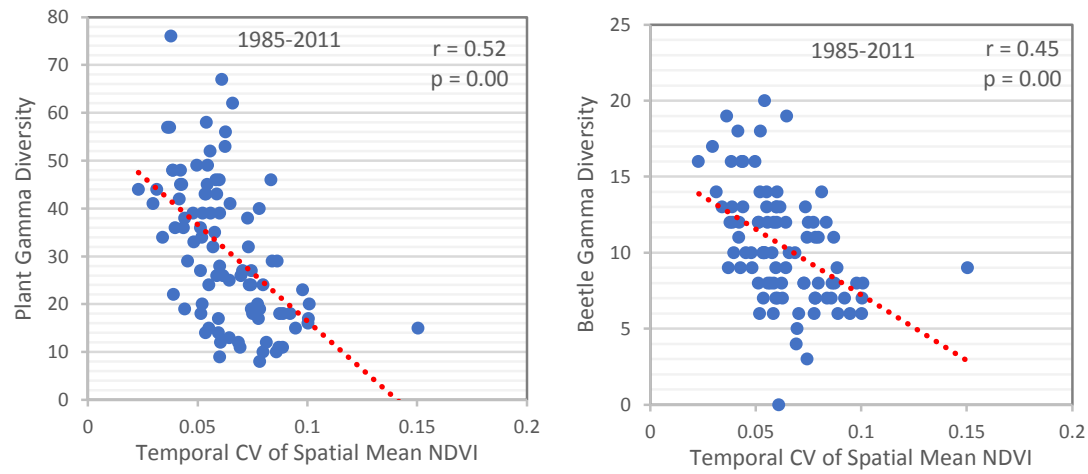

Figure 6.28. The relationship between Landsat-derived temporal CV of mean landscape NDVI for 1985-2011 and plant and beetle gamma diversity of 2011-2012.
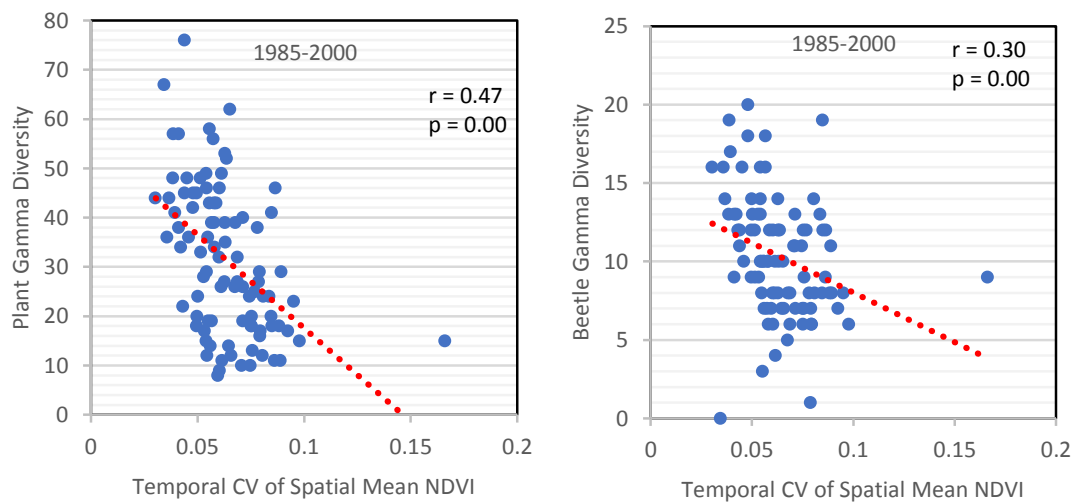

Figure 6.29. The relationship between Landsat-derived temporal CV of mean landscape NDVI for 1985-2000 and plant and beetle gamma diversity of 2011-2012.
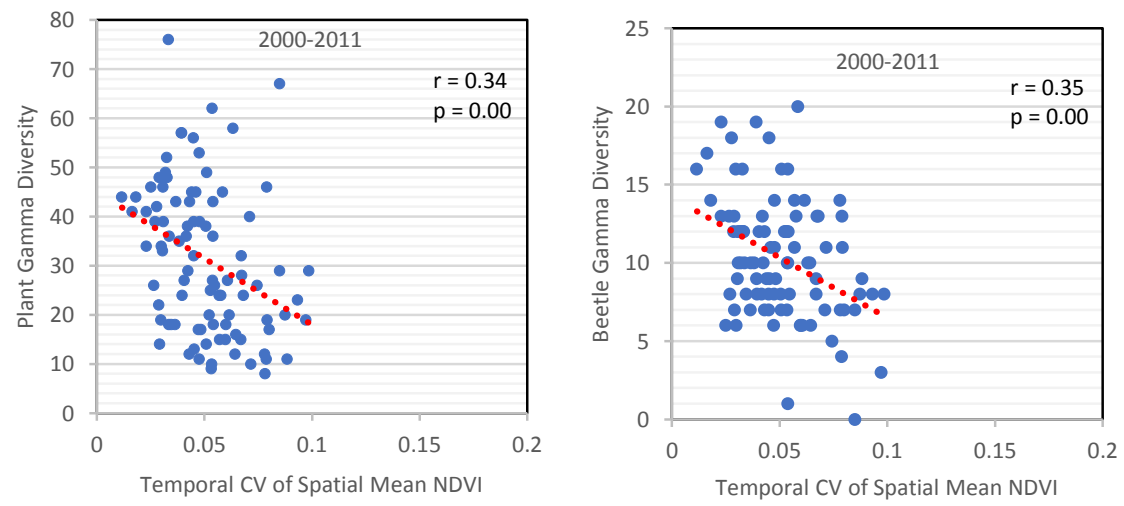

Figure 6.30. The relationship between Landsat-derived temporal CV of Mean landscape NDVI for 2000-2011 and plant and beetle gamma diversity of 2011-2012. 
Plants were the only taxon that demonstrated a significant relationship of gamma diversity with both types of temporal variables (Temporal CV of mean landscape NDVI, and the percentage of significant positively or negatively trended pixels in each sample landscape) and this occurred for the 2000-2011 Landsat NDVI time series. Plant gamma diversity of 2011-2012 was hence used in a SWR model against these three 2000-2011 temporal variables to test the relative importance of each variable in the model. The correlation matrix for these temporal explanatory variables (Table 6.6) shows that they were not highly correlated so multicollinearity was not an issue.

Table 6.6. Correlation matrix for the temporal explanatory variables.

\begin{tabular}{lrrr}
\hline & $T \_C V \_N D V I$ & \%Negative & \%Positive \\
\hline T_CV_NDVI & 1.00 & & \\
\%Negative & -0.11 & 1.00 & \\
\%Positive & -0.19 & -0.31 & 1.00 \\
\hline
\end{tabular}

The results of SWR model demonstrated a strong significant relationship $(\mathrm{r}=0.52, \mathrm{p}=$ 0.00). The percentage of significant positively trended NDVI pixels was the first variable entered $(\mathrm{r}=0.39)$, demonstrating a positive relationship with plant gamma diversity, while the temporal CV of mean landscape NDVI was the second variable entered $(r=-0.27)$, demonstrating a significant negative relationship. The results of SWR modeling were in agreement with those of bivariate modeling in showing the significant effect of percentage of significant positive trend in 2000-2011 and the temporal variability of NDVI period across landscapes in 2000-2011 on 20112012 plant gamma diversity.

\subsection{Discussion}

\subsubsection{Interpretation of main findings and relations to previous work}

The main objective of this study was to investigate land transformation and temporal changes in vegetation productivity in agricultural landscapes of eastern Ontario and their relationships with 
local farmland biodiversity measured in 2011-2012. The inter-annual landscape trends in vegetation productivity were derived from MODIS and Landsat time series and their relationships with local biodiversity were tested.

The inter-annual trends of MODIS-derived vegetation phenology and Landsat-derived TCT components were investigated independently and compared with the historic climate data such as average of July temperature, total July precipitation and the first date at which no snow on the ground was reported. The inter-annual variability in start of season, peak of season and end of season, as well as TCG and TCW were found to be related to climate and extreme seasonal weather, however, for most variables the temporal variability was not deemed to be statistically significant. Using the regional average of climate variables based on ground measurements was deemed to be appropriate for the scope of this research, since the climate of the study region did not vary significantly. Alternatively, for more detailed comparison of climate in highly variable regions climate data of each station could be used to study an area within a close proximity to each station. Using climate data derived from climate satellites such as Gravity Recovery and Climate Experiment (GRACE) is suggested for larger extent research.

For comparison purposes, July average MODIS (7-day composite) and single date July Landsat NDVI time series of the same period (2000-2011) were generated. The acquisition weeks for both data types were very close, rendering this analysis an evaluation of spatial resolution effects on trend detection and biodiversity modeling. The results showed that $61.53 \%$ of all pixels for MODIS and $70.83 \%$ of all pixels for Landsat did not experience significant increasing or decreasing trends in NDVI during this time period. When averaged to the landscape level, only 8 and 19 landscapes showed a significant positive trend in the MODIS and Landsat data, respectively, and the rest did not demonstrate a significant trend. Comparing and cross tabulating MODIS and Landsat revealed that both datasets were in general agreement in identifying $81.25 \%$ 
of pixels with no trends. While cross-tabulating MODIS and Landsat did not demonstrate a strong agreement in the proportion of significant positive and negative pixel identified by both datasets, the position and spatial distribution of some pixels with significant negative or positive trends concurred in both datasets.

Most of the pixels with significant negative trends were associated with deforestation and destruction of natural patches, as well as development of new buildings, whereas most of the pixels with significant positive trends were associated with agricultural lands. This might indicate the utilization of agricultural practices which led to increased productivity of crops or changes in the crop composition by replacing crops with lower mid-summer NDVI (such as alfalfa and winter wheat) with crops with higher mid-summer NDVI (such as corn and soybean) (Wardlow and Egbert, 2005).

The higher spatial resolution of Landsat provided more details in the spatial distribution of significant trends across the landscapes and it demonstrated higher spatial precision. This concurs with Rogers et al. (2018) who found coarser spatial resolution AVHRR and MODIS time series to be poorer than Landsat time series in portraying temporal changes and detecting early signs of tree mortality in boreal forests of North America.

In addition, since Landsat time-series were available for a longer period of time (19852011), inter-annual changes in the landscapes during this period as well as the sub periods 19852000 and 2000-2011 were analyzed. The choice of these two sub-periods was based on the observed change in the magnitude and direction of the trends slopes in most sample landscapes and for comparison purposes of MODIS and Landsat in the 2000-2011 period. It was noted that in 1985-2000 many pixels demonstrated significant positive trends while in 2000-2011 some pixels demonstrated significant negative trends. The analysis of sub periods helped to identify the period at which most of the land use transformation and decrease in vegetation biomass and 
productivity had occurred. However, multiple periods could be selected based on the observed short term (2-4 years) sub-trends to take into account the effect of crop rotation and other short term climate variations or landscape changes. In addition, arbitrary time intervals could be selected to test the effect of time series length on the trends, to identify different sub-trends with different (and significant) slopes, and to test the sensitivity of trends to the time intervals between images. For example, Czerwinski et al. (2014) divided a 20-year time series of Landsat 5 images into 1-3, 4-5 and 6-7 year intervals to analyze time series extent effects on detection of forest changes. They found that the 1-3 and a 4-5 year intervals produced similar change results, while the 6-7 year interval did not detect the same changes and suggested very little changes had occurred. They concluded that the results of the 1-3 year interval was likely the most reliable, with the 4-5 year interval being comparable to it.

The analysis of temporal changes based on landscape heterogeneity revealed that for landscape averaged NDVI all H_H, H_L, L_H and L_L landscapes demonstrated positive trends during 1985-2000. For the 2000-2011 period a disagreement between MODIS and Landsat was evident for most of the $\mathrm{H}_{-} \mathrm{H}, \mathrm{H} \mathrm{B}_{\mathrm{L}} \mathrm{L}$ and $\mathrm{L}_{-} \mathrm{H}$ landscapes as they demonstrated a positive trend with MODIS and a negative trend with Landsat. For L_L landscapes, 61.5\% and 69.2\% demonstrated a positive trend with Landsat and MODIS, respectively. Increased NDVI in low heterogeneity landscapes with low crop diversity and larger mean field size could be due to implementing intensive agricultural practices which lead to increased crop productivity or a transition to more productive crop types with greater green vegetation density.

The relationship of farmland biodiversity and MODIS and Landsat based inter-annual landscape trends were tested in this study. While MODIS-based inter-annual trends did not demonstrate a significant relationship with species diversity, significant negative relationships were found between plant and beetle gamma diversity and NDVI temporal variability (coefficient 
of variation) in the Landsat time series. In addition, plants demonstrated a negative relationship with the percentage of all pixels with negative TS slope in each sample landscape and a positive relationship with the percentage of pixels with positive TS slope in each sample landscape. Birds and butterflies did not show significant relations with the inter-annual variability of landscapes captured by either the MODIS or Landsat datasets.

The negative impacts of land use and land use change change on biodiversity in different environments have been studied in other contexts. For example, De Baan et al. (2013) found generally negative impacts of change in different land use types on biodiversity in various global regions. Jetz et al. (2007) estimated the projected impacts of climate change and land use change on global avian diversity and found that 400 species are projected to suffer by the year 2050 . These species are predominantly narrow-ranged and endemic to the tropics, where the projected range contractions are mainly driven by anthropogenic land conversion. In addition, the relationship between agricultural land use change and farmland biodiversity has been previously investigated. Examples were introduced in 6.1 (Senapathi et al., 2015; Reidsma et al., 2006; Jiang et al., 2002) that found negative impacts of land use change. However, remote sensing-based time series were not utilized in those studies and long-term temporal changes in landscape productivity derived from remote sensing data have not been largely used to model the response of local biodiversity. Further investigation using different spatial and temporal resolution remote sensing data is required to improve the methods and techniques and compare the results to those found in this study.

\subsubsection{Limitations and recommendations for future research}

Limitations in the remote sensing data of this study included the coarse spatial resolution of MODIS data as described in Chapter 4.7.2, the inconsistent availability of cloud free mid-summer Landsat data, which resulted in gap in the Landsat time series as described in Chapter 5.7.2. 
Different fusion techniques are recommended for future research to take advantage of MODIS high temporal resolution and Landsat high spatial resolution and to overcome the limitations of both datasets in portraying temporal landscape trends. As examples, the spatial and temporal adaptive reflectance fusion model (STARFM) was used by Schmidt et al. (2012) to combine MODIS and Landsat imagery to detect sudden change events in an Australian Savana, Walker et al. (2012) produced synthetic imagery of MODIS and Landsat time series to detect changes in dryland vegetation phenology in Arizona, and Hilker et al. (2009) blended MODIS and Landsat datasets to study coniferous forest phenology in central British Colombia.

Another limitation of this study was the selected biodiversity indicator (gamma diversity) and landscape metric (NDVI as an indicator of landscape productivity) for assessing the impacts of land cover change on biodiversity. In a study by Hanley et al. (2012), six indicators of bird diversity (Curlew density, Lapwing density, Skylark density, Song thrush density, Linnet density, Total density, Total richness) were modeled against different farm types with various land use (mainly used for grazing and sheep, dairy and beef cattle production) and different management practices and policy scenarios. The found that generalization of the impact of land use on biodiversity could be misleading and use of umbrella species or biodiversity indicators such as total richness can miss important species compositional effects. Other biodiversity indicators could be used in future studies to investigate the impacts of land cover change not only on the total number of species, but also on the potential shift in the species populations' composition. Use of long-term biodiversity surveys such as Breeding Bird Survey (BBS) program is recommended to monitor the changes in species total richness as well as composition over time in relation with the land cover change. In addition, comparing farmland biodiversity with biodiversity of adjacent natural and semi-natural lands is recommended to assess the changes in species richness and composition due to land conversion to agriculture. 


\subsection{Conclusion}

This study demonstrated the effect of temporal variation in vegetation productivity on farmland biodiversity. It compared the potential of MODIS and Landsat datasets in detecting the significant changes in a predominantly agricultural area of eastern Ontario and compared the performance of both datasets in detecting temporal changes. The amount the significant negative and positive trends demonstrated a general agreement between MODIS and Landsat time series, while and the spatial distribution of significant positive and negative trends was in agreement in some areas. Both datasets displayed no significant trends for the majority of pixels in all sample landscapes.

The percentage of positive, negative and no trend mid-summer Landsat NDVI pixels in each sample landscape was significantly related to plant and beetle gamma diversity while birds and butterflies did not demonstrate any significant relationships. None of the taxa showed a significant relationship with MODIS-based significant positive or negative trends in mid-summer NDVI.

Plant and beetle diversity demonstrated a significant relationship with the percentage of pixels with negative trends in the later period (2000-2011). Given that the percentage of pixels with negative trends were generally higher in the early period (1985-2000) than that of the later period, this significant relationship may indicate a time lagged response of plants and beetles to the negative changes in the landscape productivity in the early period. Most of the significant negative trends were spatially associated with natural and semi-natural patches and in some cases removal of field edges and hedge rows. Protecting these land covers in agricultural landscapes is essential for long-term support of farmland biodiversity. Besides this study, the effects of interannual changes in remote sensing-based landscape vegetation indices on local biodiversity have 
not been largely studied. More research is required to identify the effects of negative changes on biodiversity in different environments and develop new landscape management practices to provide long-term support for local species. 


\section{Chapter 7: Synthesis and major findings}

\section{1. Introduction}

The overall goal of this thesis was to investigate the response of farmland biodiversity to spatial and temporal variations in the landscape using continuous metrics of spectral heterogeneity derived from time series of high temporal and low spatial resolution imagery such as MODIS and moderate spatial and low temporal resolution imagery such as Landsat. This thesis is the first comprehensive study of the effects of landscape spatial and temporal variations on multiple taxa in a mixed agricultural region. The main research questions and objectives were presented in Chapter 1 and the background, literature and previous research on this topic were reviewed in Chapter 2. The research was implemented using data from $93,1 \times 1 \mathrm{~km}$ and $3 \times 3 \mathrm{~km}$ sample landscapes in a predominantly agricultural area in eastern Ontario as presented in detail in Chapter 3. Biodiversity data for seven taxa had been collected in 2011 and 2012 in the central $1 \times 1 \mathrm{~km}$ of these landscapes prior to this research. The overall goal of this thesis was based on the spectral heterogeneity hypothesis (Rocchini et al., 2010) and was addressed in the three empirical studies of Chapters 4, 5 and 6. In Chapter 4, seasonal vegetation phenology metrics were calculated for each year from 2000 to 2012 using MODIS NDVI time series. The spatial mean and variability of these metrics for the $1 \times 1 \mathrm{~km}$ and $3 \times 3 \mathrm{~km}$ landscapes were modeled against biodiversity of each corresponding landscape. In Chapter 5, mid-growing season Landsat time series from 1985 to 2011 were used to calculate spectral metrics of vegetation such as vegetation indices and Tasseled Cap brightness, vegetation, and moisture components. The spatial mean and variability of those metrics were extracted for the $1 \times 1 \mathrm{~km}$ and $3 \times 3 \mathrm{~km}$ sample landscapes and modeled against the local biodiversity. Finally, in Chapter 6, the inter-annual variability of the MODISderived phenological variables of Chapter 4 and the Landsat-derived mid-growing season variables of Chapter 5, as well as NDVI for equivalent July Landsat and MODIS datasets, were 
calculated. Trend analysis for these variables was performed and significant increasing and decreasing trends were identified and analyzed. Trend metrics were then modeled against local biodiversity to investigate the effects of antecedent inter-annual temporal variability of vegetation and landscape dynamics on local biodiversity measured in 2011 and 2012. The effect of spatial resolution of remote sensing data in detecting vegetation trends and biodiversity response was also investigated. This chapter summarizes and synthesizes the findings of this thesis and their relations to the literature.

\section{2. Major findings and their relation to the literature}

\subsubsection{Spectral heterogeneity hypothesis}

The spectral heterogeneity hypothesis was developed based on the ecological hypothesis that higher biodiversity is associated with more spatially heterogeneous landscapes and landscape spatial heterogeneity can be captured by continuous spectral metrics derived from satellite remote sensing (Lausch et al. 2016). Different image-based spectral metrics such as image texture variables (Duro et al., 2014; St. Louis et al., 2014), Principal Components (Rugani and Rocchini, 2017), and image-based spectral variability as a proxy of natural processes such as soil characteristics (Lausch et al., 2016) have been used as indicators of biodiversity to support the spectral heterogeneity hypothesis.

In this thesis, the spectral heterogeneity hypothesis was supported throughout Chapter 4. The spatial mean and variability $(\mathrm{CV})$ of MODIS-derived vegetation phenological metrics, especially metrics of vegetation greenness and senescence at the start and end of the growing season and their corresponding day of year, were identified as good indicators of landscape spectral heterogeneity and were found to be significantly related to local farmland biodiversity, especially for wild plants and beetles in $3 \times 3 \mathrm{~km}$ sample landscapes. In particular, the most 
common significant relationship over the years of MODIS data tested demonstrated that earlier start of season and higher vegetation productivity at the start of season is associated with higher plant diversity, and higher average of NDVI over the growing season is associated with higher beetle diversity.

The spectral heterogeneity hypothesis was also supported throughout Chapter 5. The landscape mean and spatial variability $(\mathrm{CV})$ of mid-growing season Landsat-derived spectral indices were found to be good indicators of spectral heterogeneity within and between landscapes. NDVI was used as a proxy of vegetation productivity, TCB as an indicator of soil exposure, TCG as an indicator of landscape green vegetation and TCW as an indicator of soil and vegetation moisture content. In particular, plant diversity demonstrated a significant negative relationship with TCB and all other taxa were significantly related to TCW in many years (beetles and birds negative, butterflies positive).

\subsubsection{Effects of landscape temporal dynamics of biodiversity}

This thesis investigated whether there was a time lagged response of biodiversity to the MODIS phenology and Landsat mid-growing season metrics by comparing the strength of the relationship between 2011 and 2012 biodiversity and the spectral metrics of the same years as opposed to those for previous years. No statistically significant difference in the strength of past and present models was evident for birds, beetles and butterflies although potential patterns of lag effect in the response of plants to MODIS-derived (around year 2007) and Landsat-derived (around year 1997) spectral metrics were noted.

In addition to spectral variability over the spatial dimensions of the landscapes, it was hypothesized that long-term vegetation dynamics, i.e. inter-annual temporal heterogeneity within and between landscapes, is related to local biodiversity. This hypothesis was supported in Chapter

6. The main objective of this chapter was to detect and analyze temporal landscape trends using 
mid-summer inter-annual MODIS and Landsat time series and to model characteristics of detected trends against local farmland biodiversity. Inter-annual variation of the MODIS and Landsat variables were deemed to be related to climate. Both data types demonstrated comparable results by showing no significant trends for the majority of pixels in all sample landscapes. However, the spatial distribution of pixels within landscapes with significant positive and negative trends detected by MODIS and Landsat only coincided in some areas. The July average MODIS NDVI time series was temporally consistent, while Landsat, with higher spatial resolution, was capable of portraying more spatial details and spatial precision of the trends.

Temporal trends were demonstrated to be related to landscape compositional heterogeneity (characterized by crop diversity) and configurational heterogeneity (characterized by mean field size). According to Statistics Canada, Census of Agriculture (2016), in Ontario the total number of smaller farms (under 4 ha) declined from 16,654 in 1996 to 13,193 in 2016, while the total number of larger farms (1425 ha and above) increased from 4,801 in 1996 to 9,089 in 2016. The increase in farm size was associated with removal of field edges and amalgamation of smaller fields into larger monoculture fields. This was coupled with a shift in crop composition from crops with lower mid-summer NDVI such as winter wheat, hay and alfalfa to those with higher biomass and higher mid-summer NDVI such as corn and soybean. According to Statistics Canada, Census of Agriculture (2016), the total area of tame or seeded pasture (hay, alfalfa and other perennial species) in Ontario decreased from 348,348 ha in 1996 to 208,076 ha in 2016, while the total number of soybean and corn farms increased by 400 and 800, respectively. In addition, the total area of summer fallow lands decreased from 19,624 ha in 1996 to 6,428 ha in 2016 . These temporal changes in landscapes composition and configuration were deemed to be reflected in the MODIS and Landsat time series, especially in the pixel level analysis. 
Beetles and plants were found to be significantly related to the landscape inter-annual temporal changes derived from Landsat. Plant gamma diversity was negatively related to the proportion of landscape pixels with negative vegetation trends and positively related to the proportion of landscape pixels with positive vegetation trends and no trends, while beetle diversity was positively related to the proportion of pixels with no trends. MODIS-derived temporal trends did not demonstrate significant relationships. In addition, plant and beetle gamma diversity demonstrated significant negative relationships with the temporal variability of vegetation productivity represented by the temporal CV of NDVI across the sample landscapes, i.e higher temporal variability on NDVI was associated with lower plant and beetle diversity. The effect of landscape temporal heterogeneity derived from remote sensing data on biodiversity has not been largely studied. The results of this study were promising and further investigation is recommended to study the effect of remote sensing derived temporal heterogeneity on different taxa in different ecosystems.

\subsubsection{Taxa sensitivity to temporal landscape dynamics and possible causes}

Among the seven taxa that were initially evaluated in this research, wild plants, beetles, butterflies and birds were selected for further modeling using random forest regression and SWR. Among these, beetles and wild plants showed the strongest relationships with MODIS-based and Landsatbased spectral metrics at both $1 \times 1 \mathrm{~km}$ and $3 \times 3 \mathrm{~km}$ landscape extents. In addition, as mentioned, plants and beetles were more sensitive than other taxa to the inter-annual variability of vegetation in the landscapes.

The inter-annual variability in vegetation phenology and productivity as well as the amount of bare soil and the landscape moisture content was deemed to be affected by climate factors such as temperature, precipitation and the timing of snow melt. Plant and beetle diversity were strongly related to the MODIS-derived variables of start of season, which were shown to be related to the 
time of snow melt. In addition, MODIS-derived $3 \times 3 \mathrm{~km}$ models for plants were much weaker in 2012 compared to models for the previous years, which may demonstrate the effect of low precipitation $(26 \mathrm{~mm})$ and high average July temperature $\left(22.8^{\circ} \mathrm{C}\right)$ in that year compared to the 28-year climate average. The negative relationship of plant diversity and Landsat-derived landscape brightness was also deemed to be affected by climate, since this relationship was mostly evident in the years with low July precipitation. The negative relationship of beetle diversity with Landsat-derived landscape wetness was associated with low precipitation, since this relationship was noted mostly in years with low July precipitation (except 1995 and 2007).

In addition, farming practices were considered to be an important factor affecting farmland diversity. According to Statistics Canada, Census of Agriculture (2016), the total area of farmland in Ontario that used herbicide increased from 2,000,000 ha in 1996 to 2,500,000 ha in 2016, while the average size of farms that reported herbicide use increased from 148 to 243 during this period. The total area of farmland that used insecticide also increased drastically from 370,000 ha in 1996 to $1,000,000$ ha in 2016 , while the average size of farms that reported insecticide use increased from 33 ha to 92 ha. An increase in tillage practices was also reported from 2,500,000 ha in 1996 to $3,000,000$ ha in Ontario. These practices, and increased farm size, are expected to negatively affect farmland biodiversity. In a study by Martin et al. (2018) using the same sample landscapes and biodiversity field data as this research, the negative effect of farming practices including tillage and use of herbicides on farmland biodiversity was highly significant. While plant diversity is essential for the functioning and productivity of farmlands by maintaining pollinator diversity, pest control and preserving local plant communities against invasive species (Freemark et al., 2001; Albrecht et al., 2007; Carvalheiro et al., 2011; Herzog et al., 2013), wild plants are often perceived as "weeds" by farmers. In addition, while beetle species can be natural predators of invertebrate crop pests in farmlands (Harwood et al., 2009), they are affected by pesticides, 
leading to an increase in pest numbers and causing damage to crops. Agricultural policies need to be developed and implemented to improve the awareness and understanding of farmers regarding the benefits of wild plants and natural predators such as beetles in agro-ecosystems, to encourage adoption of farming practices to preserve wild plants and beetles, and to support retention or installation of natural and semi-natural farm edges and hedgerows.

\subsubsection{Comparing to previous research}

The analysis of effects of landscape spatial and temporal variation on biodiversity using remote sensing data has not been studied, hence little literature was found to directly compare with the results of this thesis. Although the effects of natural habitat loss and fragmentation on biodiversity have been, and remain an area of interest for ecologists and natural scientists, the effects of temporal variation in vegetation productivity in highly variable landscapes such as agricultural regions on biodiversity has remained unknown. The negative effect of temporal variation in agricultural landscapes, especially a decrease in vegetation productivity, on wild plant diversity was found for the first time in this thesis.

Prior to this work, Duro et al. (2014) used the same sample landscapes and biodiversity data with Landsat-derived predictors of the productivity of cropped vegetation and the spatial variability in productivity, as well as discrete variables of composition and configuration of land cover in modeling of biodiversity. The results demonstrated significant relationships between mid-summer Mean NDVI and plant gamma diversity, and mid-summer CV of local Geary's C for NDVI of crop vegetation (as representative of variability between adjacent NDVI values) and bird gamma diversity. However, Duro et al. (2014) only modeled biodiversity data of 2011 against spectral metrics derived from mid-summer Landsat 5 data for the same year, while this study modeled biodiversity data of 2011 and 2012 against spectral metrics derived from MODIS and Landsat for those years and past years. This study concurred with Duro et al. (2014) by 
demonstrating significant relationships of spectral metrics with farmland biodiversity and added to that study by testing different spectral metrics at multiple spatial scales and for years prior to 2011-2012 and evaluating their relationships with the 2011-2012 biodiversity data.

Fahrig et al. (2015) also used the same sample landscapes and biodiversity data and modeled the Percent Crop, Log Mean Field Size and Crop Shannon Diversity Index as explanatory variables against biodiversity. The results demonstrated a significant negative relationship between Log Mean Field Size and plant and butterfly gamma diversity and between Percent Crop and beetle beta diversity. As mentioned in 7.4, field size in Ontario demonstrated an increase from 1996 to present. While this increase in the field size is a temporal process, Fahrig et al. (2015) only studied the spatial variation of mean field size between different sample landscapes for the combined years of biodiversity data collection, 2011 and 2012. Log Mean Field Size and Percent Crop both represent agricultural intensification. Although the biodiversity relationship with metrics of agricultural intensification was not directly tested in this study, temporal variations in vegetation productivity and the percentage of the area with significant positive and negative changes in NDVI were detected as proxies of agricultural intensification and negatively related to gamma diversity of plants. In addition, temporal variations in the sample landscapes were considered to be related to landscape compositional and configurational heterogeneity, with more significant negative trends noted in landscapes with low compositional and low configurational heterogeneity, i.e. more intensively used landscapes.

The strength of the models in Duro et al. (2014) and Fahrig et al. (2015) were comparable with those of this thesis, while some of the models of this study, especially MODIS-derived models for plants, were stronger. In addition, more significant models were achieved in this thesis when explanatory variables of individual multiple years were modeled against biodiversity. As mentioned, Duro et al. (2014) and Fahrig et al. (2015) were conducted using single year data, with 
spatial variations between landscapes inferred to be representative of potential temporal landscape variations. They did not incorporate analysis of actual temporal landscape dynamics and their relations with biodiversity while this thesis demonstrated significant negative relationships of inter-annual temporal variation of landscapes (vegetation NDVI and TCB) with plant gamma diversity. This indicates the importance of landscape temporal dynamics for local biodiversity, especially in highly dynamic landscapes such as farmlands.

In addition, Duro et al. (2014) and Fahrig et al. (2015) focussed on the direction of the significant relationships between their selected landscape variables and the biodiversity variables. Given that those studies were conducted at single dates, a consistent direction of the relationships was obtained, while the direction of the relationships obtained in this research were inconsistent in some cases (especially for Landsat-derived variables in Chapter 5). This inconsistent direction of the relations was one of the challenges of this research for intrepratation of the results. It could be due to inter-annual variation in climate and landscape change or the interaction between the explanatory variables which can cause a shift in the direction of the relationships. Additional alternative statistical methods which take into account the interactions between explanatory variables are suggested to overcome this limitation.

\section{3. Alternative approaches and recommendations for further research in biodiversity assessment using remote sensing.}

Alpha, beta and gamma diversity metrics were initially tested in this study. Gamma and beta diversity showed about equal potential in exploratory bivariate models of biodiversity using remote sensing data. Gamma diversity was selected for further in-depth modelling as it represents overall diversity of a landscape and is more directly linked to landscape variables extracted from imagery than beta diversity, which represents species turnover between sites in a landscape. Gamma diversity was shown in this research to be related to the remote sensing based landscape 
metrics, however, such indicators of landscape species richness might generalize the effect of spatial and temporal changes in the landscape on biodiversity and miss the effects on species composition (examples presented in Chapter 6.6.2). Alternatively, different indicators of biodiversity such as taxonomic, structural and functional diversity have been used to measure different aspects of biodiversity (Lausch et al., 2016, Schneider et al., 2017). Such indicators should be used in the future studies to investigate the impacts of spatial and temporal landscape change, not only on the total number of species in a given taxon, but also on the potential shift in the species composition in response to changes in vegetation phenology and interannual productivity.

The potential of different types of earth observation (EO) data for providing large-area, long-term, standardized, continuous, and economically feasible information for quantifying and assessing global biodiversity of different animal and plant species has largely been reviewed and emphasized (Turner et al, 2003; Nagendra et al., 2001, Pettorelli et al., 2016; Lausch et al., 2016). This thesis supported the potential of optical multispectral EO data for indirect assessment of the relationships between spatial and temporal agricultural landscape dynamics and biodiversity. Various other sensor types and methods have been proposed and developed for direct and indirect measurement of biodiversity, including more recent airborne and spaceborne sensors such as thermal, laser, radar and hyperspectral (Examples Schneider et al., 2017; Rocchini et al., 2017). While direct detection of animal species by means of remote sensing is limited to specific data types such as thermal and to very high spatial resolution imagery, plant diversity, including functional and structural diversity could be estimated using plant spectral traits and their spatial and temporal variations.

While the biodiversity data of this research is one of the best datasets collected nationally and worldwide, it was a single year dataset (46 sample landscapes surveyed in 2011 and 47 sample 
landscapes surveyed in 2012). Lack of multi-temporal biodiversity field data was one of the main limitations of this study as elaborated in Chapter 4.7.2. Biodiversity field surveys are generally challenging and collecting long-term field data could remain as a main limitation for similar research. Biodiversity surveys are restricted by several factors such as a short survey season, and climate factors such as wind, cloud, temperature and late springs (late snowmelt and low temperatures), which can affect the survival of some species in landscapes or affect the abundance and richness of species that are identified on a given day. National and continental biodiversity datasets such as the long-term archive of North America Breeding Bird Survey (BBS), as recommended in Chapter 4.7.2, could be used for similar research to avoid the temporal limitations of field surveys.

Another main challenge of this thesis was the spatial limitation of the MODIS data as described in Chapter 4.7.2. While the high temporal resolution of MODIS, coupled with weekly compositing of MODIS images was capable of capturing variability in vegetation phenology in dynamic landscapes such as farmlands, the increase in the statistical significance of $3 \times 3 \mathrm{~km}$ models compared to $1 \times 1 \mathrm{~km}$ models demonstrated that MODIS was more appropriate for larger extent analysis including regional and continental scales. For Landsat, the limited availability of cloud free mid-summer period (July-August) images as described in Chapter 5.7.2, as well as absence of Landsat 5 data for 2012 remained a significant limitation of this thesis. Different fusion techniques as described in 6.6.2 are suggested for future research to combine the high temporal resolution of MODIS with the high spatial resolution of Landsat time series. Combining MODIS and Landsat dataset would also enable using the best MODIS-derived phenological variable(s) and Landsat-derived variable(s) and creating mixed models for individual years, as well as interannual models. In addition, recently launched optical sensors such as Sentinel $2 \mathrm{a}$ and b (launched 2015 and 2017, respectively) with spatial resolutions of 10,20 and $60 \mathrm{~m}$, and a sensor revisit time 
of five days from a two-satellite constellation are recommended for future work. Combined with other sensor types such as the Radarsat Constellation of three satellites (expected launch February, 2019), will improve both the spatial and temporal resolution as well as provide different vegetation biochemical and biophysical information from the optical and microwave portions of the spectrum. Time series of these data types, once they become long enough for temporal trend analysis should improve on the methods and results of this research.

Overall, this research has shown that remote sensing is promising in portraying and quantifying landscape temporal changes in relation to local biodiversity. In addition, advancement in the technology and development of new sensors with new spectral ranges can overcome the limitations of currently available data. Further research is recommended to model biodiversity with regards to landscape dynamics and temporal variations in different environments and climate regimes.

\section{4. Implications of this research for policy and monitoring of biodiversity}

In the past few decades, the primary objective of Canadian agricultural policy has shifted from increasing output and promotion of income stability in the agriculture sector to agricultural research focusing on sustainable ways to increase productivity and limit environmental impacts (Agri-Environmental Report Series, 2016). To fulfill this objective, Agriculture and Agri-food Canada sets a series of agri-environmental indicators every year to measure the environmental performance of agriculture and the agri-food sector for soil, water, air quality and farmland management and incorporates the results in analysis of overall environmental performance. These indicators are assessed over time and areas showing improvements are highlighted, while regions which require policy interventions are identified. According to the Agri-Environmental Report 
Series (2016), the Wildlife Habitat on Farmland Index, as an indicator of biodiversity, demonstrated a decline from a "moderate' state in 1986 to a "poor" state in 2011.

Increasing landscape heterogeneity is suggested by this thesis, as well as other resources as cited in Chapter 1 and 2, to improve the capacity of farmlands for wildlife species. Long term monitoring of landscape heterogeneity using ground measurments or discrete land cover maps created by digitizing high resolution aerial photos could be costly, and time and labour intensive. Measuring landscape heterogeneity with remote sensing derived metrics of vegetation productivity and landscape conditions, as used in this research, is a time and cost-effective alternative, especially for multi-temporal and inter-annual analysis. The methods that were utilized in this research could be automated and could be used operationally for spatial and temporal monitoring of landscape heterogeneity and its relationships with local biodiversity. More research is recommended to improve the processing time and to adapt the the methods to different satellite data and various ecosystems and climates.

The results of this thesis and similar research in this field could be used to support the premise that spatial and temporsl landscape heterogeneity does affect biodiversity and this can be implemented by policy analysts and governments at local, provincial and federal levels to support farmland biodiversity. Demonstration and justification to farmers of the value of preservation or installation of natural and seminatural patches, woody field edges and hedgerows to decrease field sizes and improve connectivity and habitat, as well as the value of increasing crop diversity and avoiding monocultures are suggested as means to increase landscape heterogeneity and reinforce such agricultural policies. 


\section{References:}

Albrecht Matthias, Peter Duelli, Christine Müller, David Kleijn, and Bernhard Schmid, 2007, The Swiss agri-environment scheme enhances pollinator diversity and plant reproductive success in nearby intensively managed farmland, Journal of Applied Ecology, 44: 813-822

Anderson, D. R., 2007, Model based inference in the life sciences: A primer on evidence (1st edition). Springer (2008).

Anyamba A. and Tucker C.J., 2005, Analysis of Sahelian vegetation dynamics using MOAAAVHRR NDVI data from 1981-2003, Journal of Arid Environments, 63: 596-614

Arnett R.H. and Thomas M.C., 2000, American Beetles, Volume I: Archostemata, Myxophaga, Adephaga, Polyphaga: Staphyliniformia, CRC Press LLC, Boca Raton, FL.

Asner Gregory and Martin Robert, 2016, Spectranomics: Emerging science and conservation opportunities at the interface of biodiversity and remote sensing, Global Ecology and Conservation, 8: 212-219

Atzberger, C., \& Eilers, P. H., 2011a, Evaluating the effectiveness of smoothing algorithms in the absence of ground reference measurements, International Journal of Remote Sensing, 32(13): 3689-3709

Atkinson Peter, Jeganathan C. Dash Jadu and Atzberger Clement, 2012, Inter-comparison of four models for smoothing satellite sensor time-series data to estimate vegetation phenology, Remote Sensing of Environment, 123: 400-417

August, P.V., 1983, The role of habitat complexity and heterogeneity in structuring tropical mammal communities. Ecology 64(6): 1495-1507

Azzali, S., \& Menenti, M., 2000, Mapping vegetation-soil - climate complexes in southern Africa using temporal Fourier transform of NOAA-AVHRR NDVI data. International Journal of Remote Sensing, 21: 975-996

Baig Mohammad, Zhang Lifu, Shuai Tong and Tong Qinxi, 2014, Derivation of tasseled cap transformation based on Landsat 8 at-satellite reflectance, Remote Sensing Letters, 5(5): 432431

Bannari Abdu, Aslahi H. and Taillet Phipil, 2002, Transformed difference vegetation index (TDVI) for vegetation comer mapping, IEEE Geoscience and Remote Sensing Symposium, Vol 5

Bar-Massada Avi, Wood Eric M., Pidgeon Anna M. and Radeloff Volker C., 2011, Complex effects of scale on the relationships of landscape pattern versus avian species richness and community structure in a woodland savanna mosaic, Ecography, 35: 393-411

Beck Pieter S.A., Atzberger Clement, Høgda Kjell Arild, Johansen Bernt, Skidmore Andrew K., 2006, Improved monitoring of vegetation dynamics at very high latitudes: A new method using MODIS NDVI, Remote Sensing of Environment, 100: 321-334 
Bennett Andrew, Radford James and Haslem Angie, 2006, Properties of land mosaics:

Implications for nature conservation in agricultural environments, Biological Conservation, 133 (2): 250-264

Bennett, Joseph, R. Maloney, T. Steeves, P. Seddon, and H.P. Possingham, 2017, Spending limited resources on de-extinction could lead to net biodiversity loss, Nature Ecology and Evolution, 1: 1-4

Benton Tim, Vickery Juliet A. and Wilson Jeremy D., 2003, Farmland biodiversity: Is habitat heterogeneity the key?, Trends in Ecology and Evolution, 18 (4): 182-188

Bertrand Colette, Burel Francoise and Baudry Jacques, 2016, Spatial and temporal heterogeneity of the crop mosaic influence carabid beetles in agricultural landscapes, Landscape Ecology, 31: 451-466

Boyd D. and G. Foody, 2011, An overview of recent remote sensing and GIS based research in ecological informatics, Ecological Informatics, 6: 25-36

Breiman Leo, 2001, Random Forest, 45(1): 5-32

Brose, Ulrich, 2001, Relative importance of isolation, area and habitat heterogeneity for vascular plant species richness of temporary wetlands in east-German farmland, Ecography, 24: $722-730$

Breusch, T. S. and Pagan, A. R., 1979, A Simple Test for Heteroskedasticity and Random Coefficient Variation, Econometrica. 47 (5): 1287-1294

Buckland S.T, Magurran A.E, Green R.E and Fewster R.M, 2005, Monitoring change in biodiversity through composite indices, Philosophical Transaction of the Royal Society, 360: 243-254

Bunnell Fred and Huggard David, 1999, Biodiversity across spatial and temporal scales: problems and opportunities, Forest Ecology and Management, 115: 113-126

Burel Franciose, Butet Alain, Delettre Yannick and Millan de la Pena Norma, 2004, Differential response of selected taxa to landscape context and agricultural intensification, Landscape and Urban Planning, 67: 195-204

Burnham, K. P. and Anderson, D. R (2002) Model selection and multimodel inference: a practical information-theoretic approach. 2nd ed. New York, Springer-Verlag

Cracknell Arthur, 2001, The exciting and totally unanticipated success of the AVHRR in applications for which it was never intended, Advances in space Research, 28(1): 233-240

Carvalheiro Luı'sa Gigante, Ruan Veldtman, Awraris Getachew Shenkute, Gebreamlak Bezabih Tesfay, Christian Walter Werner Pirk, John Sydney Donaldson and Susan Wendy Nicolson, 2011, Natural and within-farmland biodiversity enhances crop productivity, Ecology Letters, 14: 251-259

Champagne, Catherin, Agriculture and Agri-food Canada, Personal communication, 2017 
Chen, J., Jönsson, P., Tamura, M., Gu, Z., Matsushita, B., \& Eklundh, L., 2004, A simple method for reconstructing a high-quality NDVI time-series data set based on the SavitzkyGolay filter, Remote Sensing of Environment, 91(3): 332-344

Chen Youhua and Shen Tsung, 2017, A general framework for predicting delayed responses of ecological communities to habitat loss, Scientific Reports, 7: 998

Chen Yaoliang, Song Xiaodong, Wang Shusen, Huang Jingfeng, Mansaray Lamin R., 2016, Impacts of spatial heterogeneity on crop area mapping in Canada using MODIS data, ISPRS Journal of Photogrammetry and Remote Sensing, 119: 451-461

Chust G., Pretus J., Ducrot D., Bedos A. and Deharveng L., 2003, Response of soil fauna to Landscape heterogeneity: Detecting optimal scales for biodiversity modeling, Conservation Biology, 17(6): 1721-1723

Committee on the Status of Endangered Wildlife in Canada (COSEWIC), 2004, https://www.newswire.ca/

Cohen Warren and Goward Samuel, 2004, Landsat's role in Ecological applications of remote sensing, BioScience, 54(6): 535-545

Cord Anna, Klein Doris, Mora Franz and Dech Stefan, 2014, Comparing the suitability of classified land cover data and remote sensing variables for modeling distribution patterns of plants, Ecological Modeling, 272: 129-140

Concepcion Elena. D., Diaz Mario and Baquero Rocio A., 2008, Effects of landscape complexity on the ecological effectiveness of agri-environment schemes, Landscape Ecology, 23: $135-148$

Costanza Jennifer. K., Moody Aaron and Peet Robert, 2011, Multi-scale environmental heterogeneity as a predictor of plant species richness, Landscape Ecology, 26: 851-864

Cottingham Kathryn, Brown Bryan and Lennon Jay, 2001, Biodiversity may regulate the temporal variability of ecological systems, 4: 72-85

Crist Eric, 1985, A TM Tasseled Cap Equivalent Transformation for Reflectance Factor Data, Remote Sensing of environment, 17: 301-306

Cutler D. Richard, Edward Thomas C., Beard Karen, Cutler Adele, Hess, Kyle, Gibson Jacob and Lawler Joshua, 2007, Random forests for classification in ecology, Ecology,88 (11): 2783 2792

Culman S. W. et al., 2010, Biodiversity is associated with indicators of soil ecosystem functions over a landscape gradient of agricultural intensification, Landscape Ecology, 25: 1333-1348

Cunningham Ross B., Lindenmayer David B., Crane Mason, Michael Damian R., Barton Philip S., Gibbons Philip, Okada Sachiko, Ikin Karen and Stein John A. R., 2014, The law of diminishing returns: woodland birds respond to native vegetation cover at multiple spatial scales and over time, Biodiversity Research, 20: 59-71 
Cushman, S.A., McGarigal, K. and Neel, M.C., 2008, Parsimony in landscape metrics: strength, universality, and consistency. Ecological Indicaators, 8, 691-703

Czerwinski Chris, King Doug and Mitchell Scott, 2014, Mapping Forest growth and decline in a temperature mixed forest using temporal trend analysis of Landsat imagery, 1978-2010, Remote Sensing of Environment, 141: 188-200

Davidson Andrew and Csillag Ferenc, 2003, A comparison of three approaches for predicting C4 species cover of northern mixed grass prarie, Remote Sensing of Environment, 86: 70-82

Davidson Andrew, Csillag Ferenc and Wilmshurt J., 2007, Diversity-productivity relations at a northern prairie site: An investigation using spectral data, Community Ecology, 8(1): 87-102

Davis C.L. Hoffman M.T. and Roberts W., 2017, Long-term trends in vegetation phenology and productivity over Namaqualand using the GIMMS AVHRR NDVI3g data from 1982 to 2011, South African Journal of Botany, 111: 76-85

De Beurs Kirsten and Henebry Geoffrey, 2010, Spatio-Temporal statistical methods for modelling land surface phenology, Phenological Research, Chapter 9, Springer:177-208

De Baan Laura, Alkemade Rob and Koellner Thomas, 2013, Land use impacts on biodiversity in LCA: a global approach, International Journal of Life Cycle Assessment, 18: 1216-1230

De Simon Serena, Sigura Maurizia and Boscutti Francesco, 2017, Patterns of Biodiversity and Habitat sensitivity in agricultural landscapes, Journal of Environmental Planning and Management, 60 (7): 1173-1192

DeVries Philip and Walla Thomas, 1999, Species diversity in spatial and temporal dimensions of fruit-feeding butterflies from two Ecuadorian rainforests, Biolodical Journal of the Linnean Society, 68: 333-353

Diamond JM., 1989, Overview of recent extinctions, Western D, Pearl MC, editors. Conservation for the Twenty-First Century. Oxford: Oxford University Press: 37-41

Donald, Paul, Sanderson Fiona, Burfield Ian and van Bommel Frans, 2006, Further evidence of continent-wide impacts of agricultural intensification on European farmland birds, 1990-2000, Agriculture, Ecosystems and Environment, 116 (3)L 189-196

Dong Taifeng, Liu Jiangui, Shang Jiali, Qian Budong, Huffman Ted, Zhang Yinsuo, Champagne Catherine and Daneshfar Bahram, 2016, Assessing the Impact of Climate Variability on Cropland Productivity in the Canadian Prairies Using Time Series MODIS FAPAR, Remote Sensing, 8: 281

Doraiswamy P. C. Hatfield J.L, Jackson T.J., Akhmedov B., Prueger J., Stern A., 2004, Crop condition and yield simulations using Landsat and MODIS, Remote Sensing of Environment, 92: $548-559$

Dornelas Maria, Magurran Anne E., Buckland Stephen T., Chao Anne, Chazdon Robin L., Colwell Robert K., Curtis Tom, Gaston Kevin J., Gotelli Nicholas J., Kosnik Matthew 
A., McGill Brian, McCune Jenny L., Morlon Hélène, Mumby Peter J., Øvreås Lise, Studeny Angelika, and Vellend Mark, 2013, Quantifying temporal change in biodiversity: challenges and opportunities, Proceeding Biological Science, 280(1750): 20121931

Debinski D.M. Kindscher K. and Jakubauskas M. E., 1999, A remote sensing and GIS-based model of habitats and biodiversity in the Greater Yellowstone Ecosystem, International Journal of Remote Sensing, 20 (17): 3281-3291

Dubovyk Olena, Landmann Tobias, Erasmus Barend F.N., Tewes Andreas, Schellberg Jürgen, 2015, Monitoring vegetation dynamics with medium resolution MODIS-EVI time series at subregional scale in southern Africa, International Journal of Applied Earth Observation and Geoinformation, 38: 175-183

Duelli Peter and Obrist Martin, 2003, Biodiversity indicators: the choice of values and measures, Agriculture, Ecosystems and Environment, 98: 87-98

Duflot Remi, Aviron Stephan, Ernoult Aud, Lenore Fahrig, Burel Francoise, 2015, Reconsidering the role of "semi-natural habitat" in agricultural landscape biodiversity: a case study, Ecological Research, 30: 75-83

Duflot Remi, Ernoult Aud, Aviron Stephan, Lenore Fahrig, Burel Francoise, 2017, relative effects of landscape composition and configuration on multi-habitat gamma diversity in agricultural landscapes, Agriculture, Ecosystem and Environment, 241: 62-69

Dullinger Stefan et al., 2012, Extinction debt of high-mountain plants under twenty-first-century climate change, Nature Climate Change, 2: 619-622

Duro Denis, Coops Nicholas and Wulder Michael, 2007, Development of a large area biodiversity monitoring system driven by remote sensing, Progress in Physical Geography, 31(3): $235-260$

Duro Denis, Franklin Steven ad Dube Monique, 2012, A comparison of pixel-based and objectbased image analysis with selected machine learning algorithms for the classification of agricultural landscapes using SPOT-5 HRG imagery, Remote Sensing of Environment, 118: $259-272$

Duro Denis, Girard Jude, King Douglas, Fahrig Lenore, Mitchell Scott, Lindsay Kathryn and Tischendorf Lutz, 2014, Predicting species diversity in agricultural environments using Landsat TM imagery, Remote Sensing of Environment, 144: 214-225

Dungan J. L., Perry N.J, Dale M.R.T., Legendre P., Citron S., Fortin M.J, Jakomulska A., Miriti M. and Rosenberg M.S, 2002, A balanced view of scale in spatial statistical analysis, Ecography, 25: 625-640

Dubovyk Olena, Landmann Tobias, Erasmus Barend and Schellberg Jurgen, 2015, Monitoring vegetation dynamics with medium resolution MODIS-EVI time series at sub-regional scale in southern Africa, International Journal of Applied Earth Observation and Geoinformation, 38:175-183 
Eckert Sandra, Hüsler Fabia, Liniger Hanspeter, Hodel Elias, 2015, Trend analysis of MODIS NDVI time series for detecting land degradation and regeneration in Mongolia, Journal of Arid Environment, 113: 16-28

Eilers, P. H., 2003, A perfect smoother, Analytical Chemistry, 75(14): 3631-3636

Essl Franz, Dullinger Stefan, Rabitsch Wolfgang, Hulme Philip E., Pys Petr, Wilson John R.U., and Richardson David M., 2015, Delayed biodiversity change: no time to waste, Trends in Ecology and Evolution, 30(7)

Fang Xiuqin, Zhu Qiuan, Ren Liliang, Chen Huai, Wang Kai, Peng Changhui, 2018, Largescale detection of vegetation dynamics and their potential drivers using MODIS images and BFAST: A case study in Quebec, Canada, Remote Sensing of Environment, 206: 391-402

Fahrig Lenore, 2003, Effects of Habitat Fragmentation on Biodiversity, Annual Review of Ecology, Evolution and Systematics, 34:487-515

Farhig Lenore, Baudry Jacques, Brotons Lluís, Burel Françoise G., Crist Thomas O., Fuller Robert J., Sirami Clelia, Siriwardena Gavin M., Martin Jean- Louis, 2011, Functional landscape heterogeneity and animal biodiversity in agricultural landscapes, Ecological Letters, 14:101-112

Fahrig, Lenore, 2013, Rethinking patch size and isolation effects: the habitat amount hypothesis, Journal of Biogeography, 40: 1649-1663.

Fahrig, Lenore, Girard Jude, Duro Dennis, Pasher Jon, Smith Adam, Javorek S., King Doug, Freemark Lindsay Kathryn, Mitchell Scot and Tischendorf Lutz, 2015. Farmlands with smaller crop fields have higher within-field biodiversity. Agriculture, Ecosystems, and Environment. 200: $219-234$

Feng Quanlong, Gong Jianhua, Liu Jiantao and Yi Li, 2015, Monitoring Cropland Dynamics of the Yellow River Delta based on Multi-Temporal Landsat Imagery over 1986 to 2015, Sustainability, 7: 14834-14858

Fensholt Rasmus, Rasmussen Kjeld, Nielsen Thomas Theis and Mbow Cheikh, 2009, Evaluation of earth observation based long term vegetation trends - Intercomparing NDVI time series trend analysis consistency of Sahel from AVHRR GIMMS, Terra MODIS and SPOT VGT data, Remote Sensing of Environment, 113: 1886-1898

Fensholt Rasmus and Proud Simon, 2012, Evaluation of Earth Observation based global longterm vegetation trends-Comparing GIMMS and MODIS global NDVI time series, Remote Sensing of Environment, 119: 131-147

Filippi-Codaccioni Ondine, Devictor Vincent, Bas Yves and Julliard Romian, 2010, Toward more concern for specialization and less for species diversity in conserving farmland biodiversity, Biological Conservation, 143: 1493-1500

Findlay Scott and Bourdages Josee, 1999, Response time of wetland biodiversity to road construction on adjacent lands, Conservation Biology, 14 (1) 86-94 
Fisher Jermy and Mustard John, 2007, Cross-scalar satellite phenology from ground, Landsat and MODIS data, Remote Sensing of Environment, 109: 261-273

Fissette Thierry, Rollin Patrick, Campbell Leander, Davidson Andrew and Daneshfar Bahram, 2012, AAFC annual crop inventory, status and challenges, Agricultural and Agri-food Canada Publication

Flick, Tatiana, Feagan Sean and Fahrig Lenore, 2012, Effects of landscape structure on butterfly species richness and abundance in agricultural landscapes in eastern Ontario,

Canada. Agriculture, Ecosystems and Environment. 156: 123-133

Foody Giles. M. 2002, Status of land cover classification accuracy assessment, Remote Sensing of Environment, 80(1): 185-201

Foody Giles. M. 2010, Assessing the accuracy of land cover change with imperfect ground reference data, Remote Sensing of Environment, 114: 2271-2285

Forman Richard and Deblinger Robert, 2001, The ecological road-effect zone of Massachusetts suburban highways, Conservation biology, 14(1): 36-46

Franklin, Jerry, 1988, Structural and functional diversity in temperate forests, Pages 166-175 in E. 0. Wilson, editor. Biodiversity. National Academy Press, Washington, D.C

Franklin Steven E., 2009. Remote Sensing for Biodiversity and Wildlife Management: Synthesis and Applications. McGraw-Hill, Whitby Ont.

Freemark Kathryn, Boutin Celine, Keddy Cathy, 2002, Importance of farmland habitats for conservation of plant species, Conservation Biology, 16(2): 399-412

Fuhlendorf Samuel, Harrell Wade, Engle David, Hamilton Robert, Davis Craig and Leslie David, 2006, Should heterogeneity be the basis for conservation? Grassland bird response to fire and grazing, 16 (5) 1706-1716

Galbraith, H., 1988, Effects of agriculture on the breeding ecology of lapwings Vanellus vanellus. Journal of Applied Ecology, 25: 487-503

Gallardo Belinda, Gascon Stephanie, Quintana Xavier, and Comin Francisco, 2011, How to choose a biodiversity indicator - Redundancy and complementarity of biodiversity metrics in a freshwater ecosystem, Ecological Indicators, 11(5): 1177-1184

Gamez-Virues S., Perovic D.J, Gossner M.M., Borschig C., Bluthgen N., de Jong H., et al., 2015, Landscape simplification filters species traits and drives biotic homogenization, Nature Communications, 6: 8568

Genuer Robin, Poggi Jean and Tuleau Christine, 2010, Variable Selection using Random forest, Patter Recognition Letters, 31(14): 2225-2236

Ghermandi Andrea, Ding Helen and Nunes Paulo, 2013, The social dimension of biodiversity policy in the European Union: Valuing the benefits to vulnerable communities, Environmental Science and Policy, 33: 169-208 
Ghimire B., 2010, Contextual land-cover classification: incorporating spatial dependence in land-cover classification models using random forests and the Getis statistic, Remote Sensing Letters, 1(1):45-54

Gillanders Steve N., Coops Nicholas C., Wulder Michael, A., Gergel Sarah E. and Nelson Trisalyn, 2008, Multitemporal remote sensing of landscape dynamics and pattern change: describing natural and anthropogenic trends, Progress in Physical Geography, 32(5): 503-528

Gillespie Thomas, Foody Giles M and Rocchini Duccio, 2008, Measuring and modelling biodiversity from space, Progress in Physical Geograph, 32: 203-221

Gislason Pall Oskar, Benediktsson Jon and Sveinsson Johannes, 2006, Random Forests for land cover classification, Pattern Recognition Letters 27: 294-300

Gómez Cristina, 2011, Characterizing the state and processes of change in a dynamic forest environment using hierarchical spatio-temporal segmentation, Remote Sensing of Environment, 115: $1665-1679$

Gómez Cristina, White Joanne and Wulder Mitchael, 2014, Historical forest biomass dynamics modeled with Landsat spectral trajectories, ISPRS Journal od Photogrammetry and Remote Sensing, 93: 14-28

Gotelli Nicholas and Colwell Roert, Quantifying biodiversity: Procedures and pitfalls in the measurement and comparison of species richness, Ecology Letters, 4: 379-391

Gottschalk, T.K., Huettmann, F. and Ehler, M., 2005, Thirty years of analyzing and modeling avian habitat relationships using satellite imagery: a review, International Journal of Remote Sensing, 26: 2631-56

Gromping Ulrike, 2009, Variable Importance Assessment in Regression: Linear Regression versus Random Forest, The American Statistician, 63(4): 308-319

Guay Kevin C., Beck Pieter S. A., Berner Logan T., Goetz Scott J., Baccini Alessandro, and Buermann Wolfgang, 2014, Vegetation productivity patterns at high northern latitudes: a multisensor satellite data assessment, Global Change Biology, 20: 3147-3158

Guralnick, R.P., Hill, A.W., Lane, M., 2007, Towards a collaborative global infrastructure for biodiversity assessment. Ecology Letters, 8: 663-672.

Guyot Claire, Arlettaz Raphae, Korner Pius, Jacot Alain, 2017, Temporal and Spatial Scales Matter: Circannual Habitat Selection by Bird Communities in Vineyards, Plos One, 12(2): 0170176.

Hahs AK, McDonnell MJ, McCarthy MA, Vesk PA, Corlett RT, Norton BA, et al., 2009, A global synthesis of plant extinction rates in urban areas. Ecology Letters, 12:1165-1173

Haila, Y., 2002, A conceptual genealogy of fragmentation research: from island biogeography to landscape ecology. Ecological Applications, 12, 321-334.

Hald, A.B., 1999, Weed vegetation (wild flora) of long established organic versus conventional cereal fields in Denmark. Annals of Applied Biology 134, 307-314 
Hanley Nick, Acs Szvetlana, Dallimer Martin, Gaston Kevin J., Graves Anil, Morris Joe, Armsworth Paul R., 2012, Farm-scale ecological and economic impacts of agricultural change in the uplands, Land Use Policy, 29: 587-597

Hansen, Brigitte, Fjelsted Hugo and Kristensn Steen, 2001, Approaches to assess the environmental impact of organic farming with particular regard to Denmark. Agricultural Ecosystems and Environments 83, 11-26

Hao Pengyu, Wang Li, Zhan Yulin, Niu Zheng and Wu Mingquan, 2016, Using historic NDVI time-series to classify crops at $30 \mathrm{~m}$ spatial resolution: A case on southern Kansas, IGRASS Proceeding, 6316-6319

Harwood James D., Phillips Sarah W., Lello Joanne, Sunderland Keith D., Glen David M., Bruford Michael W., Harper Georgina L., Symondson William O.C., 2009, Invertebrate biodiversity affects predator fitness and hence potential to control pests in crops, Biological Control, 51: 499-506

Harrell, F. E. Jr., Lee, K. L., Califf, R. M., Pryor, D. B., Rosati, R. A., 1984, Regression modelling strategies for improved prognostic prediction, Stat Med, 3 (2): 143-52

Hatfield Jerry and Prueger John, 2010, Value of Using Different Vegetative Indices to Quantify Agricultural Crop Characteristics at Different Growth Stages under Varying Management Practices, Remote Sensing, 2: 562-578

Hebblewhite Mark, Merrill Evelyn and McDermid Greg, 2008, A multi-scale test of the forage maturation hypothesis in a partially migratory ungulate population, Ecological Monograph, 78(2): 141-166

Haberl Helmut, Schulz Niels B., Christoph Plutzar, Karl Heinz Erb, Fridolin Krausmann, Wolfgang Loibl, Dietmar Moser, Norbert Sauberer, Helga Weisz, Harald G. Zechmeister, Peter Zulka, 2004, Human appropriation of net primary production and species diversity in agricultural landscapes, Agriculture, Ecosystems and Environment, 102: 213-218

Herzog Felix and Jeanneret Philippe, 2013, Measuring farmland biodiversity, Solutions, 4(4)

Herrando Sergi, Anton Marc, Sardà-Palomera Francesc, Bota Gerard, Gregory Richard D. and Brotons Lluís, 2014, Indicators of the impact of land use changes using large-scale bird surveys: Land abandonment in a Mediterranean region, Ecological Indicators, 45: 235-244

Higgins Mark A, Asner Gregory P., Perez Eneas, Elespuru Nydia, Tuomisto Hanna, Ruokolainen Kalle and Alonso Alfonso, 2012, Use of Landsat and SRTM Data to Detect BroadScale Biodiversity Patterns in Northwestern Amazonia, Remote Sensing, 4: 2401-2418

Hilker Thomas, Wulder Michael, Coops Nicholas, Seitz Nicole, White Joanne, Gao Feng, Masek Jeffrey, Stenhouse Gordon, 2009, Generation of dense time series synthetic Landsat data through data blending with MODIS using a spatial and temporal adaptive reflectance fusion model, Remote Sensing of Environment, 113: 1988-1999

Hilker Thomas, Wulder Michael, Coops Nicholas, Linke Julia, McDermid Greg, Masek Jeffrey, Gao Feng, White Joanne, 2009, A new data fusion model for high spatial and temporal 
resolution mapping of forest distribution based on Landsat and MODIS, Remote Sensing of Environment, 113, 1613-1627

Hirosawa Yuji, Marsh Stuart and Kilman Douglas, 1996, Application of Standardized principal component analysis of land cover characterization using multi temporal AVHRR data, Remote Sensing of Environment, 38(3): 267-281

Hoaglin, D., Mosteller, F., and Tukey, J., 2000, Understanding Robust and Exploratory Data Analysis (285-294). New York: John Wiley.

Holben, B. N., 1986, Characteristics of maximum-value composite images from temporal AVHRR data, International Journal of Remote Sensing, 7(11): 1417-1434.

Huang Chengquan, Goward Samuel N., Masek Jeffrey G., Thomas Nancy, Zhu Zhiliang and Vogelmann James E., 2010, An automated approach for reconstructing recent forest disturbance history using dense Landsat time series stacks, Remote Sensing of Environment, 114: 183-198

Huete A.R., 1988, A soil-adjusted vegetation index (SAVI), Remote Sensing of Environment, 25(3): 295-309

Hurlbert Allen and Haskell John, 2003, The effect of energy and seasonality on avian species richness and community composition, The American Naturalist, 161(1): 83-97

Huttich Christian, Herold Martin, Stdohbach Ben and Dech Stefan, 2011, Integrating in-situ, Landsat, and MODIS data for mapping in Southern African savannas: experiences of LCCSbased land-cover mapping in the Kalahari in Namibia, Environment Monitoring Assesment, 176: $531-547$

Hylander Kristoffer and Johan Ehrlén, 2013, The mechanisms causing extinction debts, Trends in Ecology and Evolution, 28(6): 341-346

Ichii K., Kawabata A. and Yamaguchi Y., 2002, Global correlation analysis for NDVI and climate variables and NDVI trends: 1982-1990, International Journal of remote Sensing, 23 (18): $3873-3878$

Jakubauskas ME, Legates DR and Kastens JH, 2001, Harmonic analysis of time-series AVHRR NDVI data. Photogrammetry Engineering and Remote Sensing 67:461-470

Javorek S. K., Antonowitsch R., Callaghan C., Grant M., and Weins T., 2006, Changes to wildlife habitat on agricultural lands in Canada 1981-2001, Canadian Journal of Soil Science, 87: $225-233$

Jensen J. R, 1996, Introductory Digital Image Processing, Prentic Hall

Jönsson P. and Eklundh L., 2002, Seasonality extraction by function fitting to time-series of satellite sensor data. IEEE Geoscience and Remote Sensing 40:1824-1831

Kauth R.J. and Thomas G.S., 1976, The Tasseled Cap: A Graphic Description of the SpectralTemporal Development of Agricultural Crops as Seen by LANDSAT, Symposium on Machine Processing of Remotely Sensed Data, Paper 159 
Jetz Walter, Wilcove David S., and Dobson Andrew P., 2007, Projected Impacts of Climate and Land-Use Change on the Global Diversity of Birds, Plos Biology, 5(6): e157

Jiang Yuan, Kang Muyi, Gao Qingzhu, He Lihuan, Xiong Min, Jia Zhibin And Jin Zhengping, 2003, Impact of land use on plant biodiversity and measures for biodiversity conservation in the Loess Plateau in China - a case study in a hilly-gully region of the Northern Loess Plateau, Biodiversity and Conservation, 12: 2121-2133

Jones H. G. and Vaughan R. A., 2010, Remote Sensing of Vegetation, Oxford University Press Ju Junchange and Masek Jeffrey, 2016, The vegetation greenness trend in Canada and US Alaska from 1984-2012 Landsat data, Remote Sensing of Environment, 146: 1:16

Kaennel Dobbertin, M., 1998, Indicators for forest biodiversity in Europe: Proposal for terms and definitions. BEAR Technical Report No. 4.

Karlson Marten, Mortberg Ulla and Balfors Berit, 2014, Road ecology in environmental impact assessment, Environmental Impact Assessment Review, 48: 10-19

Katayama Naoki, Amano Tatsuya, Naoe Shoji, Yamakita Takehisa, Komatsu Isamu, Takagawa Shin-ichi, Sato Naoto, Ueta Mutsuyuki, Miyashita Tadashi, 2014, Landscape heterogeneitybiodiversity relationship: effect of range size, Plos One, 9(3): e93359

Kennedy Robert, Yang Zhiqiang and Cohen Warren, 2010, Detecting trends in forest distrivbution and recovery using yearly Landsat time-series: LandTrendr-Temporal segmentation algorithms, Remote Sensing of Environment, 114: 2897-2910

Kleijn D., Kohler F., Baldi A., Batary P., Concepcion E. D., Clough Y., Diaz M., Gabriel D., Holzschuh A., Knop E., Kovacs A., Marshall E. J. P., Tscharntke T. and Verhulst J., 2009, On the relationship between farmland biodiversity and land-use intensity in Europe, Proceedings of the Royal Society, 276: 903-909

Kleijn David, Rundlo Maj, Scheper Jeroen, Smith Henrik G. and Tscharntke Teja, 2011, Does conservation on farmland contribute to halting the biodiversity decline? Trends in Ecology and Evolution, 26 (9): 474-481

Korner C., 1996, Scaling from species to vegetation: the usefulness of functional groups. In: Schulze E-D, Mooney HA, editors. Biodiversity and Ecosystem Function. New York: SpringerVerlag, 117-40.

Krauss Jochen, Bommarco Riccardo, Moisès Guardiola, Risto K Heikkinen,4 Aveliina Helm,5 Mikko Kuussaari, Regina Lindborg, Erik Öckinger, Meelis Pärtel, Joan Pino,3 Juha Pöyry, Katja M Raatikainen, Anu Sang, Constantí Stefanescu, Tiit Teder, Martin Zobel, and Ingolf Steffan-Dewenter, 2010, Habitat fragmentation causes immediate and timedelayed biodiversity loss at different trophic levels, Ecology Letters, 13(5): 597-605

Kuenzer Claudia. Ottinger Marco, Wegmann, Guo Huadong, Wang Changlin, Zhang Jianzhong, Dech Stefan and Wikelski Martin, 2014, Review Article: Earth observation satellite sensors for biodiversity monitoring: potentials and bottlenecks, International Journal of Remote Sensing, 35(18): 6599-6647 
Laspanora Rosa and Masini Nicola, 2012, Improving satellite Quickbird-based identification of landscape archaeological features through Tasseled Cap Transformation and PCA.

Latifovic Rasim, Fytas Kostas, Chen Jing and Paraszszak Jacek, 2005, Assessing land cover change resulting from large surface mining development, International Journal of Applied Earth Observation and Geoinformation, 7: 29-48

Latifovic Rasim and Pouliot Darren, 2014, Monitoring cumulative long-term vegetation changes over the Athabasca oil sand region, IEEE Journal of Selected Topics in Appied Earth Observation and Remote Sensing, 1939-1404

Lausch Angela, Bannehr L., Beckmanna M., Boehm C., Feilhauerd H., Hacker J.M., Heurich M., Jungg A., Klenke R., Neumann C., Pausek M., Rocchini D., Schaepman M.E., Schmidtlein S., K.Schulzo, Selsam P., Settelep J., Skidmore A.K., Cord A.F., 2016, Linking Earth Observation and taxonomic, structural and functional biodiversity: Local to ecosystem perspectives, Ecological Indicators, 70: 317-339

Lee Myung and Martin James, 2017, Avian species and functional diversity in agricultural landscapes: Does landscape heterogeneity matter? Plos One, 10: 1371

Liao Chenhua, Feng Zhiming, Li Peng, Zhang Jinghua, 2015, Monitoring the spatio-temporal dynamics of swidden agriculture and fallow vegetation recovery using Landsat imagery in northern Laos, Journal of Geographical Sciences, 25(10): 1218-1234

Liaw Andy and Wiener Matthew, 2002, Classification and Regression by randomForest, 2(3): $18-22$

Lieth, H. 1974. Purposes of a Phenology Book. In: Lieth, H. (ed.) Phenology and Seasonality Modeling, Springer-Verlag, New York, NY.

Lindenmayer David B., Likens Gene E., Andersen Alan, Bowman David, Bull Michael, Burns Emma, Dickman Chris R., Hoffmann Ary A., Keith David A., Liddell Michael J., Lowe Andrew J., Metcalfe Daniel J., Phinn Stuart R., Russell-Smith Jeremy, Thurgate Nikki and Wardle Glenda M., 2012, Value of long-term ecological studies, Austral Ecology 37: 745-757

Liu Qingsheng, Liu Gaohuan, Huang Chong and Xie Chuanjie, 2015, Comparison of tasseled cap transformations based on the selective bands of Landsat 8 OLI TOA reflectance images, International Journal of Remote Sensing, 36(2): 417-441

Lovejoy, T.E., 1980, in: Barney, G.O. (Ed.), The Global 2000 Report to the President the Technical Report, vol. 2. Penguin, 327-332

Lunetta, R. S., Knight, J. F., Ediriwickrema, J., Lyon, J. G., \& Worthy, L. D., 2006, Land-cover change detection using multi-temporal MODIS NDVI data. Remote Sensing of Environment, 105(2): 142-154.

MacArthur R. H. and Wilson E. O., 1967, The theory of island biogeography, Princeton University Press, Princeton, NJ

Magurran Anne E., Baillie Stephen R., Buckland Stephen T., Dick Jan McP., Elston David A., E. Marian Scott, Smith Rognvald I., Somerfield Paul J. and Watt Allan D., 2010, Long-term 
datasets in biodiversity research and monitoring: assessing change in ecological communities through time, Trends in Ecology and Evolution 25: 574-582

Martınez-Casasnovas Jos'e A., Mart'in-Montero Almudena, and Casterad M. Auxiliadora, 2005, Mapping multi-year cropping patterns in small irrigation districts from time-series analysis of Landsat TM images, European Journal of Agronomy, 23: 159-169

Maselli Fabio, 2004, Monitoring forest conditions in a protected Mediterranean coastal area by the analysis of multiyear NDVI data, Remote Sensing of Environment, 89: 423-433

Mattison Elizabeth and Norris Ken, 2005, Bridging the gap between agricultural policy land use and biodiversity, Trends in Ecology and Evolution, 20(11): 610-616

McCloy K.R. and Lucht W., 2004, Comparative evaluation of seasonal patterns in long time series of satellite image data and simulations of a global vegetation model, IEEE Transactions on Geoscience and Remote Sensing, 42(1): 140-153

McInnes William S., Smith Brent, and McDermid Gregory J., 2015, Discriminating Native and Nonnative Grasses in the Dry Mixedgrass Prairie with MODIS NDVI Time Series, Journal of Selected Topics in Applied Earth Observations and Remote Sensing, 8(4): 1395-1403

Millard Koreen and Richardson Murray, 2015, On the importance of training data sample selection in random forest image classification: A case study in peatland ecosystem mapping, Remote Sensing, 7: 8489-8515

Mišurec Jan, Kopacková Veronika, Lhotáková Zuzana, Campbell Petya and Albrechtová Jana, 2016, Detection of Spatio-Temporal Changes of Norway Spruce Forest Stands in Ore Mountains Using Landsat Time Series and Airborne Hyperspectral Imagery, Remote Sensing, 8: 92

Mkhabelaa M.S., Bullock P., Raj S., Wang S., Yang Y., 2011, Crop yield forecasting on the Canadian Prairies using MODIS NDVI data, Agricultural and Forest Meteorology, 151: 385393

Monck-Whipp Liv, Martin Amanda E., Francis Charles M., Fahrig Lenore, 2018, Farmland heterogeneity benefits bats in agricultural landscapes, Agriculture, Ecosystems and Environment, 253: 131-139

Morelli Federico, Leszek Jerzak, Piotr Tryjanowski, 2014, Birds as useful indicators of high nature value (HNV) farmland in Central Italy, Ecological indicators, 38: 236-242

Moreno Claudia, and Halffter Gonzalo, 2001, Spatial and temporal analysis of $\alpha, \beta$ and $\gamma$ diversities of bats in a fragmented landscape, Biodiversity and Conservation 10: 367-382

Moser B.W., Pipas M.J., Witmer G.W. and Engman R.M., 2002, Small mammal use of hybrid popular plantation relative to stand age, Northwest Science, 76: 158-165

Nagendra Harini, 2001, Review article: Using remote sensing to assess biodiversity, International Journal of Remote Sensing, 22 (12): 2377-2400 
Nagendra Harini, Lucas Richard, Honradoc Joao Pradinho, Rob H.G. Jongman, Tarantino Cristina, Adamo Maria and Mairota Paola, 2013, Remote sensing for conservation monitoring: Assessing protected areas, habitat extent, habitat condition, species diversity, and threats, Ecological Indicators, 33: 45-59

Neeti N. and Eastman J. R., 2011, A Contextual Mann-Kendall Approach for the Assessment of Trend Significance in Image Time Series, Transactions in GIS, 15(5): 599-611

Nelson A. Oberthur T. and Cook S., 2007, Multi-scale correlations between topography and vegetation in a hillside catchment of Honduras, International Journal of Geographical Information Science, 21 (2): 145-174

Norris K., (2008) Agriculture and biodiversity conservation: opportunity knocks, Conservation Letters, Vol. 1, No. 1, pp. 2-11

Nguyen Thanh Son, Bui Xuan Thanh and Chau Thi Da, 2016, Monitoring Mangrove Forest Changes from Multi-temporal Landsat Data in Can Gio Biosphere Reserve, Vietnam, Wetland, 36: $565-576$

Nichol Janet E., Wong Man, Corlett Richard and Nichol Douglas, 2010, Assessing avian habitat fragmentation in urban areas of Hong Kong ta high spatial resolution using spectral unmixing, Landscape and Urban Planning, 95: 54-60

Nielsen Scott, Haughland Diane, Bayne Erin and Schick Jim, 2009, Capacity of large-scale, long-term biodiversity monitoring programs to detect trends in species prevalence, biodiversity Conservation, 18: 2961-2978

Niti Mishra, Cres Kelley, Neeti Neeti, Meyer Thoralf and Young Kenneth, 2015, MODIS derived vegetation greenness trends in African Savanna: Deconstructing and localizing the role of changing moisture availability, fire regime and anthropogenic impact, Remote Sensing of Environment, 169: 192-204

Nitze Ingmar, Barrett Brian and Cawkwell Fiona, 2014, Temporal optimization of image acquisition for land cover classification with Random Forest and MODIS time-series, International Journal of Applied Earth Observation and Geoinformation, 34: 136-146

Noss Reed, 1990, Indicators of monitoring biodiversity, A hierarchical approach, Conservation Biology, 4(4): 355-364

Oliver Tom, Roy David, Hill Jane, Brereton Tom and Thomas Chris, 2010, Heterogeneous landscapes promote population stability, Ecology Letters, 13: 473-484

Ostman, O. Ekbom Barbara, Bengtsson Janne and Weibull Ann-Christin, 2001, Landscape complexity and farming practice influence the condition of polyphagous carabid beetles. Ecology Application, Vol. 11, pp. 480-488

Oteros Jose, Garcia-Mozo Hermania, Botey Roser, Mstre Antonio and Galan Carmen, 2015, Variation in cereal crop phenology in Spain over the last twenty six years (1986-2012), Climate Change, 130: 545-558 
Palmer, M. W., 2000, Opportunities for long-term ecological research at the Tall grass Prairie Preserve, Oklahoma. In: Lajtha, K., Vanderbilt, K. (Eds.), Cooperation in Long Term Ecological Research in Central and Eastern Europe: Proceedings of ILTER Regional Workshop, Budapest, Hungary, 22-25 June, 1999, 123-128.

Pan Zhuokun, Huang Jingfeng, Zhou Qingbo, Wang Limin, Cheng Yongxiang, Zhang Hankui, Blackburn George Alan, Yan Jing, Liu Jianhong, 2015, Mapping crop phenology using NDVI time series derived from HJ-1 A/B data, International Journal of Applied Earth Observation and Geoinformation, 24: 188-197

Panda Sudhanshu, Ames Daniel and Panigrahi Suranjan, 2010, Application of Vegetation Indices for Agricultural Crop Yield Prediction Using Neural Network Techniques, Remote Sensing, 2: 673-696

Park Taejin, Ganguly Sangram, Tømmervik Hans, Euskirchen Eugénie S, Høgda Kjell-Arild, Karlsen Stein Rune, Brovkin Victor, Nemani Ramakrishna R and Myneni Ranga B, 2016, Changes in growing season duration and productivity of northern vegetation inferred from longterm remote sensing data, Environmental Research Letters, 11: 084001

Pasher Jon. King Doug and Lindsay Kathryn, 2007, Modeling and mapping potential hooded warbler (wilsonia citrina) habitat using remotely sensed imagery, Remote Sensing of Environment, 107: 471-483

Pasher, Jon, Mitchell Scott, King Doug, Fahrig Lenore, Smith Adam, and Lindsay Kathryn, 2013, Optimizing landscape selection for estimating relative effects of landscape variables on ecological responses, Landscape Ecology. 28(3): 371-383

Parmentier, Benoit., 2014, Characterization of Land Transitions Patterns from Multivariate Time Series Using Seasonal Trend Analysis and Principal Component Analysis. Remote Sensing. 6. 12639-12665. 10.3390/rs61212639

Pasquarella Valerie J., Holden Christopher E., Kaufman Les and Woodcock Curtis E., 2016, From imagery to ecology: leveraging time series of all available Landsat observations to map and monitor ecosystem state and dynamics, Remote Sensing of Ecology and Conservation, doi: $10.1002 /$ rse 2.24

Pearson D.1., Knisley C.B., Duran D.P. Kazilek C.J., 20015, A field guide to the tiger beetles of the United States and Canada: identification, natural history and distribution, Oxford Univessity Prexx, Sofia, pp. 400

Perry Eileen M., Morse-McNabb Elizabeth M., Nuttall James G., O’Leary Garry J., and Clark Robert, 2014, Managing Wheat from Space: Linking MODIS NDVI and Crop Models for Predicting Australian Dryland Wheat Biomass, Journal of Selected Topics In Applied Earth Observations And Remote Sensing, 7(No): 3724-3731

Peters Jan, Baets Bernard, Verhoest Niko, Samson Roeland, Degroeve, Becker Piet and Huybrechts Willy, 2007, Random forests as a tool for ecohydrological distribution modelling, Ecological Modelling, 207: 304-318 
Pérez-Vega Azucena, Mas Jean-François and Ligmann-Zielinska Arika, 2012, Comparing two approaches to land use/cover change modeling and their implications for the assessment of biodiversity loss in a deciduous tropical forest, Environmental Modelling and Software, 29: 1123

Petrou Zisis, Manakos Ioannis and Stathaki Tania, 2015, Remote sensing for biodiversity monitoring: a review of methods for biodiversity indicator extraction and assessment of progress towards international targets, Biodiversity Conservation, 24: 2333-2363

Pettorelli Nathalie, Vik Jon, Mysterud Atle, Gaillard Jean, Tucker Compton, Steneth Nils, 2005, Using the satellite-derived NDVI to assess ecological responses to environmental change, Trends in Ecology and Evolution, 20: 503-510

Pettorelli Nathalie, Safi Kamran and Turner Woody, 2014, Satellite remote sensing, biodiversity research and conservation of the future, Philosophical Transitions of the Royal Society

Pielou E.C., 1966, The Measurement of diversity in different types of biological collections, Journal of Theoritical Biology, 13: 131-144

Piles M., Chaparro D., Entekhabi D., Konings A.G., Jagdhuber T. and Camps G., 2017, Remote sensing of vegetation dynamics in agro-ecosystems using SMAP vegetation optical depth and optical vegetation indices, IGARSS Proceeding, 4376-4349

Poschlod P., Bakker J.P., Kahmen S., 2005, Changing land use and its impact on biodiversity, Basic and Applied Ecology, 6: 93-98

Potapov Peter, Hansen Matthew C., Stehman Stephen V., Loveland Thomas R. and Pittman Kyle, 2008, Combining MODIS and Landsat imagery to estimate and map boreal forest cover loss, Remote Sensing of Environment, 112: 3708-3719

Pouliot Darren, Latifovic Rasim, Fernandes Richard and Olthof Ian, 2011, Evaluation of compositing period and AVHRR and MERIS combination for improvement of spring phenology detection in deciduous forest, Remote Sensing of Environment, 115: 158-166

Pouliot Darren, Latifovic Rasim and Olthof Ian, 2009, Trends in vegetation NDVI from $1 \mathrm{~km}$ AVHRR data over Canada for the period 1985-2006, International Journal of Remote Sensing, 30 (1): $149-168$

Power Ryan, Coops Nicholas, Morgan Jessica, Wulder Michael, Nelson Trisalyn, Drever Charles and Cumming Steven, 2012, A remote Sensing Approach to biodiversity assessment and regionalization of the Canadian boreal forest, Progress in Physical Geography, 37(1): 36-62

Qi Yue, Fang Shibo and Wang Runyuan, 2017, Agricultural drought monitoring in Shanxi by using temperature vegetation dryness index, $15^{\text {th }}$ International Conference on Environmental Science and Technology, 00632

Ray, T. W., 1994, “A FAQ on Vegetation in Remote Sensing.” Accessed March 23, 2014. http://www. yale.edu/ceo/Documentation/rsvegfaq.html. 
Reddy Sudhaker, Jha C.S and Dadhwal V.K., 2016, Assessment and monitoring of ling term forest cover changes (1920-2013) in Western Ghats biodiversity hotspot, Journal of Earth System and Science, 125 (1): 103-114

Reed Bradley C., Brown Jesslyn F, VanderZee Darrel, Loveland Thomas R., Merchant James W. and Ohlen Donald, 1994, Measuring phenological variability from satellite imagery, Journal of Vegetation Science, 5: 703-714

Reed Bradley, 2006, Trend Analysis of time-series phenology of North America derived from satellite data, GIScience \& Remote Sensing, 43(1): 24-38

Rees W. G., 2013, Physical Principles of Remote Sensing, Cambridge University Press

Reidsma Pytrik, Tekelenburg Tonnie, van den Berg Maurits, Alkemade Rob, 2006, Impacts of land-use change on biodiversity: An assessment of agricultural biodiversity in the European Union, Agriculture, Ecosystems and Environment 114: 86-102

Rocchini Duccio, Balkenhol Niko, Cartercd Gregory A., Foody Giles M., Gillespie Thomas W., He Kate S., Kark Salit, Levini Noam, Lucas Kelly, Luoto Miska, Nagendra Harini, Oldeland Jens, Ricottao Carlo, Southworth Jane, Neteler Markus, 2010, Remotely sensed spectral heterogeneity as a proxy of species diversity: Recent advances and open challenges, Ecological Information, 5: 318-329

Rocchini Duccio, Stefanoni Jose and He Kate, 2015, Advancing species diversity estimate by remotely sensed proxies: A conceptual review, Ecological Information, 25: 22-28

Rocchini Duccio, Boyd Doreen, Feret Jean, Fooyd Giles M., He Kate, Lausch Angela, Nagendra Harini, Wegmann Martin and Pettorelli Nathalie, 2016, Satellite remote sensing to monitor species diversity: potential and pitfalls, Remote Sensing in Ecological Conservation, doi: $10.1002 /$ rse2.9

Roder Achim, Udelhoven Thomas, Hill Joachim, Tsiourlis Georgios, 2008, Trend Analysis of Landsat TM and ETM+ imagery to monitor grazing impact in a rangeland ecosystem in North Greece, Remote sensing of Environment, 112: 2863-2875

Rodriguez V.F., Abarca-Hernandez A., Ghimirie B., Chica-Olmo M., Atkinson P.M., and Jeganathan C., 2011, Incorporating Spatial Variability Measures in Land-cover Classification using Random Forest, Procedia Environmental Sciences 3: 44-49

Rodriguez V.F., Chica-Olmo M., Abarca-Hernandez A., Atkinson P.M., Jeganathan C., 2012, Random Forest classification of Mediterranean land cover using multi-seasonal imagery and multi-seasonal texture, Remote Sensing of Environment, 121:93-107

Rodriguez V.F., Ghimirie B., Rogan J., Chica-Olmo M., Rigol-Sanchez J.P., 2012, An assessment of the effectiveness of a random forest classifier for land-cover classification, ISPRS Journal of Photogrammetry and Remote Sensing, 67: 93-104

Rogers Brendan M., Solvik Kylen, Hogg Edward H., Ju Junchang, Masek Jeffrey G., Michaelian Michael, Berner Logan T., Goetz Scott J., 2018, Detecting early warning signals of 
tree mortality in boreal North America using multiscale satellite data, Global Change Biology, 24: $2284-2304$

Rondeaux Genevieve, Steven Micheal and Baret Fredric, 1996, Optimization of soil-adjusted vegetation indices, Remote Sensing of Environment, 55(2): 95-107

Rose Robert A., Byler Dirck, Eastman J. Ron, Fleishman Erica, Geller Gary, Goetz Scott, Guild Liane, Hamilton Healy, Hansen Matt, Headley Rachel, Hewson Jennifer, Ned Horning, Beth A. Kaplin, Nadine Laporte, Allison Leidner, Peter Leimgruber, Jeffrey Morisette, John Musinsky, Lilian Pintea, Ana Prados, Volker C. Radeloff, Mary Rowen, Sassan Saatchi, Steve Schill, Karyn Tabor, Turner Woody, Vodacek Anthony, Vogelmann James, Martin Wegmann, Wilkie David, and Wilson Cara, 2014, Ten ways remote sensing can contribute to conservation, Conservation Biology, 29 (2) 350-359

Rugani Benedetto and Rocchini Duccio, 2017, Boosting the use of spectral heterogeneity in the impact assessment of agricultural land use on biodiversity, Journal of Cleaner Produciton, 140: 516-524

Rydberg, N.T. and Milberg, P. (2000) A survey of weeds in organic farming in Sweden. Biol. Agric. Hortic. 18, 175-185

Sakamoto Toshihiro, Yokozawa Masayuki, Toritani Hitoshi, Shibayama Michio, Ishitsuka Naoki, Ohno Hiroyuki, 2005, A crop phenology detection method using time-series MODIS data, Remote Sensing of Environemtn, 96, 399-374

Sales Lilian Patricia, Matthew Warrington Hayward, Ludimilla Zambaldi, Marcelo Passamani, Fabiano Rodrigues de Melo, Rafael Loyola, Time-lags in primate occupancy: a study case using dynamic models, 2015, Brazilian Journal of Nature Conservation, 13: 139-144

Senapathi Deepa, Carvalheiro Luisa G., Biesmeijer Jacobus C., Dodson Cassie-Ann, Evans Rebecca. L., McKerchar Megan, Morton R. Daniel, Moss Ellen D., Stuart P. M. Roberts, Kunin William E. and Potts Simon G., 2015, The impact of over 80 years of land cover changes on bee and wasp pollinator communities in England, Proceeding Royal Society, 282: 20150294

Schlagel, J.D., \& Newton, C. M., 1996, A GIS-based statistical method to analyze spatial change, Photogrammetric Engineering and Remote Sensing, 62(7): 839-844

Schmidt Micheal, Udelhoven Thomas, Roder Achim and Gill Tony, Long term data fusion for a dense time series analysis with MODIS and Landsat imagery in an Australian Savana, 2012, Journal of Applied Remote Sensing, 6(1): 063512

Shao Yang, Lunetta Ross S., Wheeler Brandon, Iiames John S., Campbell James B., 2016, An evaluation of time-series smoothing algorithms for land-cover classifications using MODISNDVI multi-temporal data, Remote Sensing of Environment, 174: 258-265

Sheeren D., Bonthoux S. and Balent G., 2014, Modeling bird communities using unclassified remote sensing imagery: Effects of the spatial resolution and data period, Ecological Indicators, 43: $69-82$

Simpson E. H., 1949, Measurement of diversity, Nature 163:688 
Sing Ashbindu, 1989, Review Article Digital change detection techniques using remotelysensed data, International Journal of Remote Sensing, 10(6): 989-1003

Silva A., Freitas L. and Pires P., 2014, A Fournier Index upgrade as a new approach for quantitative phenological studies in plant communities, Tropical Ecology, 55(1): 137-142

Skakun Sergii, Franch Belen, Vermote Eric, Roger Jean-Claude, Becker-Reshef Inbal, Justice Christopher, Kussul Nataliia, 2017, Early season large-area winter crop mapping using MODIS NDVI data, growing degree days information and a Gaussian mixture model, Remote Sensing of Environment, 195: 244-258

Smart Simon, Bunce Robert, Firbank Lex and Coward Paul, 2002, Do field boundaries act as refugia for grassland plant species diversity in intensively managed agricultural landscapes in Britain? Agriculture, Ecosystems and Environment, 91: 73-87

Strayer, D., 1.S. Ghtzenstein, e.G. Jones, 1. Kolasa, G.E. Likens, M.J. McDonnell, G.G. Parker, and S.T.A. Pickett, 1986, Long-term ecological studies: an illustrated account of their design, operatIOn, and importance to ecology. Occasional Publication of the Institute of Ecosystem Studies, Number 2, Millbrook, New York, 2: 1-38.

Steckel Julian, Westphal Catrin, Marcell Peters, Ballach Michaela, Rothenqoehrer Christoph, Erasmi Stefan, Scherber Chrisoph, Tscharntkle Teja and Steffan Ingolf, 2014, Landscape composition and configuration differently affect trap-nesting bees, wasps and their antagonists, Biological Conservation, 172: 56-64

Stefanov W. L. and Netzband M., 2005, Assessment of ASTER land cover and MODIS NDVI data at multiple scales for ecological characterization of an arid urban center, Remote Sensing of Environment, 99: 31-43

Steven Michael, Malthus Timothy, Baret Fredric, Xu Hui and Chopping Mark, 2003, Intercalibration of vegetation indices from different sensor systems, Remote Sensing of Environment, 88: 412-422

Stockli R. and Vidale P., 2004, European plant phenology and climate as seen in a 20-year AVHRR land-surface parameter dataset, International Journal of Remote Sensing, 25(17): 3303-3330

St-Louis, Ve'ronique, Pidgeon, Anna M., Radeloff, V. C., Hawbaker, T. J., \& Clayton, Murray K., 2006, High-resolution image texture as a predictor of bird species richness, Remote Sensing of Environment, 105(4): 299-312

St-Louis Ve'ronique, Pidgeon Anna M., Kuemmerle Tobias, Sonnenschein Ruth, Radeloff Volker C., Clayton Murray K., Locke Brian A., Bash Dallas and Hostert Patrick, 2014, Modelling avian biodiversity using raw, unclassified satellite imagery, Philosophical Translation of Royal Society B, 369: 20130197

Sulla-Menashe Damien, Friedl Mark and Woodcock Curtis, 2016, Sources of bias and variability in long-term Landsat time series over Canadian boreal forests, Remote Sensing of Environment, 177: 206-219 
Sunderland, K. and Samu, F., 2000, Effects of agricultural diversification on the abundance, distribution, and pest control potential of spiders: a review. Entomologia experimentalis et Applicata, 95: 1-13

Swift M.J., Izac A. M. van Noordwijk, 2004, Biodiversity and ecosystem services in agricultural landscapes - are we asking the right questions? Agriculture, Ecosystems and Environment, Vol. 104, pp. 113-134

Symondson W.O., Sunderland K.D. and Greenstone M.H., 2002, Can generalist predators be effective biocontrol agents?, Annual Review of Entomology, 47: 561-594

Tateishi R, Ebata M., 2004, Analysis of phenological change patterns using 1982-2000 Advanced Very High Resolution Radiometer (AVHRR) data. International Journal of Remote Sensing 25: 2287-2300

Teyssèdre A. Couvet D., 2007, Expected impact of agriculture expansion on the world avifauna, $C$. R. Biologies, Vol. 330, pp. 247-254

Tews, J., Brose U., Grimm V., Tielborger K., Wichmann M.C., Schwager M., and Jeltsch F., 2004, Animal species diversity driven by habitat heterogeneity/diversity: The importance of keystone structures. Journal of Biogeography 31: 79-92.

Thompson Jeffery A. and Paull David J., 2017, Assessing spatial and temporal patterns in land surface phenology for the Australian Alps (2000-2014), Remote Sensing of Environment, 199: $1-13$

Tilman David, May Robert, Lehman Clarence and Nowak Martin, 1994, Habitat destruction and the extinction debt, International Journal of Science, 371: 65-66

Tomaselli V., Adamo M., Veronico G., Sciandrello S., Tarantino C., Dimopoulos P., Medagli P., Nagendra H., and Blonda P., 2017, Definition and application of expert knowledge on vegetation pattern, phenology, and seasonality for habitat mapping, as exemplified in a Mediterranean coastal site, Plant Biosystems - An International Journal Dealing with all Aspects of Plant Biology, 151 (5): 887-899

Tottrup Christian and Rasmussen Michael Schultz, 2004, Mapping long-term changes in savannah crop productivity in Senegal through trend analysis of time series of remote sensing data, Agriculture, Ecosystems and Environment, 103: 545-560

Tucker, C. J., 1979, Red and photographic infrared linear combinations for monitoring vegetation. Remote Sensing of Environment, 8: 127-150.

Turner Woody, Spector Sacha, Gardiner Ned, Fladeland Matthew, Sterling Eleanor and Steininger Marc, 2003, Remote sensing for biodiversity science and conservation, Trends in Ecology and Evolution, 18(6): 306-314

Turner Woody, Rondinini C., Pettorelli N., Mora B., Leidner A.K., Szantoi Z., Buchanan G., Dech S., Dwyer J., Herold M., Koh L.P., Leimgruber P., Taubenboeck H., Wegmann M. Wikelskim M., Woodcock C., 2015, Free and open-access satellite data are key to biodiversity conservation, Biological Conservation, 182: 173-176 
Tuanmu Mao, Viña N., Bearer A., Xu S., Ouyang W., Zhang Z., \& Liu J., 2010, Mapping understory vegetation using phenological characteristics derived from remotely sensed data. Remote Sensing of Environment, 114: 1833-1844

Tuanmu Mao and Jetz Walter, 2015, A global, remote sensing-based characterization of terrestrial habitat heterogeneity for biodiversity and ecosystem modelling, Global Ecology and Biogeography, 24: 1329-1339

Tuomisto H., 2010, A diversity of beta diversities: straightening up a concept gone awry. Part 1. Defining beta diversity as a function of alpha and gamma diversity, Ecography 33: 2-22

Vasseur, C., Joannon, A., Aviron, S., Burel, F., Meynard, J.-M. \& Baudry, J., 2013, The cropping systems mosaic: how does the hidden heterogeneity of agricultural landscapes drive arthropod populations? Agriculture, Ecosystems \& Environment, 166, 3-14

Veech J.A. Summerville K.S., Crist T.O., Gering J.C, 2002, The additive partitioning of species diversity: recent revival of an old idea, Oikos, 93 (1): 3-9

Verbesselt Jan, Hyndman, Rob, Newnham Glenn and Culvenor Darius, 2010, Detecting trend and seasonal changes in satellite image time series, Remote Sensing of Environment, 114: 106115

Vieira Matheus Alves, Formaggio Antonio Roberto, Rennó Camilo Daleles, Atzberger Clement, Aguiar Daniel Alves, Mello Marcio Pupin, 2012, Object Based Image Analysis and Data Mining applied to a remotely sensed Landsat time-series to map sugarcane over large areas, Remote Sensing of Environment, 123: 553-562

Viña Andrés, Tuanmu Mao-Ning, Xu Weihua, Li Yu, Qi Jiaguo, Ouyang Zhiyun, Liu Jianguo, 2012, Relationship between floristic similarity and vegetated land surface phenology: Implications for the synoptic monitoring of species diversity at broad geographic regions, Remote Sensing of Environment, 121: 488-496

Viña Andrés, Liu Wei, Zhou Shiqiang, Huang Jinyan and Liu Jianguo, 2016, Land surface phenology as an indicator of biodiversity pattern, Ecological Indicators, 64: 281-288

Vogelmann James E., Xian George, Collin Homer, Brian Tolk, 2012, Monitoring gradual ecosystem change using Landsat time series analyses: Case studies in selected forest and rangeland ecosystems, Remote Sensing of Environment, 122: 92-105

Walker J.J., de Beurs K.M., Wynne R.H. and Gao, F., 2012, Evaluation of Landsat and MODIS data fusion products for analysis of dryland forest phenology, Remote Sensing of Environment, 117: $381-393$

Wang Li, Suhong Liu, Lizhao Wang, Xiaojia Liang, 2013, Spatial Distribution of Crofton Weeds in Southwest China with MODIS NDVI of Long Time Series, Geoinfomatics, 10: 278285

Wang Li, Shi C., Diao C., Ji W., and Yin D., 2016, A survey of methods incorporating spatial information in image classification and spectral unmixing, International Journal of Remote Sensing, 37(16): 3870-3910 
Wang Siyuan, Yang Bojuan, Yang Qichun, Lu Linlin, Wang Xiaoyue, Peng Yaoyao, 2016, Temporal trends and spatial variability of vegetation phenology over the Northern hemisphere during 1982-2012, Plos One, 0157134

Wagner Helen, Wildi Otto and Ewald Lkaus, 2000, Additive partitioning of plant species diversity in an agricultural mosaic landscape, Landscape Ecology, 15: 219-227

Eardlo Brian and Egbert Stephen, 2005, State-level crop mapping in the US Central Great Plains agroecosystem using MODIS 250-meter NDVI data, Global Priorities in Land Remote Sensing

Warren Steven D., Holbrook Scott W., Dale Debra A., Whelan Nathaniel L., Elyn Martin, Grimm Wolfgang, and Jentsch Anke, 2007, Biodiversity and the heterogeneous disturbance regim on military training lands, Restoration Ecology, 15 (4): 606-612

Warren Steven D., Alt Martin, Olson Keith D., Irl Severin D.H., Steinbauer Manuel J., Jentsch Anke, 2014, The relationship between the spectral diversity of satellite imagery, habitat heterogeneity, and plant species richness, Ecological Informatics, 24: 160-168

White Michael A., KiRSTENM . D E Beursw, Kamel D Idanz, Davi D W. Inouy, Andrew D. R I Chardson, Olaf P. Jensenk, John O’ Keefe, Gong Zhang, Ramakrishna R. Nemaniw W, Willem J. D. Va N Leeuwenzz, Jess Lyn F. Brown, Allard D E Wit, Mi Chael Schaepman, Xioamao Link, Michael Dettinger, Amey S. Baileyw W W, John Kimball, Mark D. Schwartz, Denni S D. Baldocchi, John T. Leek and Wi Lli Am K. Lauenroth, 2009, Intercomparison, interpretation, and assessment of spring phenology in North America estimated from remote sensing for 1982-2006, Global Change Biology, 15: 2335-2359

Whittaker, R.H., 1960, Vegetation of the Siskiyou Mountains, Oregon, and California. Ecological Monographs, 30: 279-338.

Wilson Scott, Mitchell Gregory W., Pasher Jon, McGovern Mark, Hudson Marie-Anne R., Fahrig Lenore, 2017, Influence of crop type, heterogeneity and woody structure on avian biodiversity in agricultural landscapes, Ecological Indicators, 83: 218-226

Xie Y., et al., 2008, Remote sensing imagery in vegetation mapping: a review, Journal of Plant Ecology, 1 (1): 9-23

Yang Wentao, Ming Wang, and Peijun Shi, 2013, Using MODIS NDVI Time Series to Identify Geographic Patterns of Landslides in Vegetated Regions, Geoscience and Remote Sensing Letters, 10(4) :707-710

Yeates, G.W., Bardgett R>D., Cook R., Hobbs P., Bowling P. and Potter F.,1997, Faunal and microbial diversity in three Welsh grassland soils under conventional and organic management regimes. Journal of Applied Ecology, 34: 453-470

Youhua Chen and Tsung-Jen Shen, 2017, A general framework for predicting delayed responses of ecological communities to habitat loss, Scientific Reports, 7, article number 998 (2017) 
Zamora Jorge, Verdu Jose' R., Eduardo Galante, 2007, Species richness in Mediterranean agroecosystems: Spatial and temporal analysis for biodiversity conservation, Biological Conservation 134: 113-121

Zeng Linglin, Wardlow Brian D., Wang Rui, Shane Jie, Tadesse Tsegaye, Hayes Michael J. and Li Deren, 2016, A hybrid approach for detecting corn and soybean phenology with time-series MODIS data, Remote Sensing of Environment, 181: 237-250

Zhao Jianjun, Zhang Hongyan, Zhang Zhengxiang, Guo Xiaoyi, Li Xuedong and Chen Chun, 2015, Spatial and Temporal Changes in Vegetation Phenology at Middle and High Latitudes of the Northern Hemisphere over the Past Three Decades, Remote Sensing, 7: 10973-10995

Zhu Zhe, 2017, Change detection using Landsat time series: A review of frequencies, preprocessing, algorithms, and applications, ISPRS Journal of Photogrammetry and Remote Sensing, 130: 370-384

Zongyao Sha, Zhong Jialin, Bai Yongfei, Tan Xicheng and Li Jonathan, 2016, Spatio-temporal patterns of satellite-derived grassland vegetation phenology from 1998 to 2012 in Inner Mongolia, China, Journal of Arid Land, 8(3): 462-477 
Appendices Appendix I. List of species identified in the 2011 and 2012 field surveys as presented in
Fahrig et al. (2015).

\section{Birds}

\begin{tabular}{|c|c|}
\hline Common name & Scientific name \\
\hline Alder flycatcher & Empidonax alnorum \\
\hline American crow & Corvus brachyrhynchos \\
\hline American goldfinch & Spinus tristis \\
\hline American robin & Turdus migratorius \\
\hline Baltimore oriole & Icterus galbula \\
\hline Bank swallow & Riparia riparia \\
\hline Barn swallow & Hirundo rustica \\
\hline Black-capped chickadee & Poecile atricapillus \\
\hline Blue jay & Cyanocitta cristata \\
\hline Bobolink & Dolichonyx oryzivorus \\
\hline Brown thrasher & Toxostoma rufum \\
\hline Brown-headed cowbird & Molothrus ater \\
\hline Cedar waxwing & Bombycilla cedrorum \\
\hline Chipping sparrow & Spizella passerina \\
\hline Common grackle & Quiscalus quiscula \\
\hline Common raven & Corvus corax \\
\hline Common yellowthroat & Geothlypis trichas \\
\hline Cooper's hawk & Accipiter cooperii \\
\hline Eastern kingbird & Tyrannus tyrannus \\
\hline Eastern meadowlark & Sturnella magna \\
\hline Eastern phoebe & Sayornis phoebe \\
\hline Eastern wood-pewee & Contopus virens \\
\hline European starling & Sturnus vulgaris \\
\hline Great crested flycatcher & Myiarchus crinitus \\
\hline Grasshopper sparrow & Ammodramus savannarum \\
\hline Gray catbird & Dumetella carolinensis \\
\hline Gray partridge & Perdix perdix \\
\hline Hairy woodpecker & Picoides villosus \\
\hline Horned lark & Eremophila alpestris \\
\hline Indigo bunting & Passerina cyanea \\
\hline Killdeer & Charadrius vociferus \\
\hline Mourning dove & Zenaida macroura \\
\hline Northern cardinal & Cardinalis cardinalis \\
\hline Northern flicker & Colaptes auratus \\
\hline Pine siskin & Spinus pinus \\
\hline Red-eyed vireo & Vireo olivaceus \\
\hline Red-winged blackbird & Agelaius phoeniceus \\
\hline Ring-billed gull & Larus delawarensis \\
\hline Rock pigeon & Columba livia \\
\hline Rose-breasted grosbeak & Pheucticus ludovicianus \\
\hline Savannah sparrow & Passerculus sandwichensis \\
\hline Short-eared owl & Asio flammeus \\
\hline Song sparrow & Melospiza melodia \\
\hline Spotted sandpiper & Actitis macularius \\
\hline Swamp sparrow & Melospiza georgiana \\
\hline Tree swallow & Tachycineta bicolor \\
\hline Upland sandpiper & Bartramia longicauda \\
\hline Vesper sparrow & Pooecetes gramineus \\
\hline Warbling vireo & Vireo gilvus \\
\hline White-breasted nuthatch & Sitta carolinensis \\
\hline Wilson's snipe & Gallinago delicate \\
\hline Yellow warbler & Setophaga petechia \\
\hline
\end{tabular}


Plants

\begin{tabular}{|c|c|}
\hline Common name & Scientific name \\
\hline Alfalfa & Medicago sativa \\
\hline Alsike clover & Trifolium hybridum \\
\hline Annual blue grass & Роа аппиа \\
\hline Annual fleabane/daisy fleabane & Erigeron annuus \\
\hline Annual sow-thistle & Sonchus oleraceus \\
\hline Aster spp. & Asteraceae spp. \\
\hline Avens spp. & Avens spp. \\
\hline Awlfruit sedge & Carex stipata \\
\hline Barnyard grass & Echinochola crusgalli \\
\hline Bird rape & Brassica campestris \\
\hline Bird's foot trefoil & Lotus corniculatus \\
\hline Bittersweet nightshade & Solanum dulcamara \\
\hline Black medic & Medicago lupulina \\
\hline Bladder campion & Silene vulgaris \\
\hline Blue-eyed grass & Sisyrinchium spp. \\
\hline Bluegrass sp. & Poa spp. \\
\hline Boneset & Eupatorium spp. \\
\hline Brassicaceae spp. & Brassicaceae spp. \\
\hline Bristly foxtail & Setaria verticillata \\
\hline Brown-eyed susan & Rudbeckia hirta \\
\hline Bull thistle & Cirsium vulgare \\
\hline Canada anemone & Anemone canadensis \\
\hline Canada fleabane & Erigeron canadensis \\
\hline Canada goldenrod & Solidago canadensis \\
\hline Canada thistle & Cirsium arvense \\
\hline Chickweed & Stellaria media \\
\hline Chicory & Cichorium intybus \\
\hline Cleavers & Galium aparine \\
\hline Common Burdock & Arctium minus \\
\hline Common milkweed & Asclepias syriaca \\
\hline Common mullein & Verbascum thapsus \\
\hline Common peppergrass & Lepidium densiflorum \\
\hline Common yellow oxalis & Oxalis stricta \\
\hline Corn spurry & Spergula arvensis \\
\hline Crabgrass spp. & Digitaria spp. \\
\hline Creeping buttercup & Ranunculus repens \\
\hline Creeping charlie & Nepeta hederacea \\
\hline Curled dock & Rumex crispus \\
\hline Dandelion & Taraxacum officinale \\
\hline Eastern black nightshade & Solanum ptycanthum \\
\hline Elecampane & Inula helenium \\
\hline Epilobium spp. & Epilobium spp. \\
\hline Evening primrose & Oenothera biennis \\
\hline Fall dandelion/fall hawkbit & Leontodon autumnalis \\
\hline Fall panicum & Panicum dichotomiflorum \\
\hline Fern spp. & Fern spp. \\
\hline Field bindweed & Convolvulus arvensis \\
\hline Field horsetail & Equisetum arvense \\
\hline Galium spp. & Galium spp. \\
\hline Giant foxtail & Setaria faberii \\
\hline Goat's beard & Tragopogon dubius \\
\hline Grass-leaved stitchwort & Stellaria graminea \\
\hline Green foxtail & Setaria viridis \\
\hline Green pigweed & Amaranthus powellii \\
\hline Hairy galinsoga & Galinsoga ciliata \\
\hline Heal-all/selfheal & Prunella vulgaris \\
\hline Inland rush & Juncus interior \\
\hline Jewelweed/spotted touch-me-not & Impatiens capensis \\
\hline Kentucky bluegrass & Poa pratensis \\
\hline Lady's thumb & Polygonum persicaria \\
\hline Lamb's quarters & Chenopodium album \\
\hline Large crab grass & Digitaria sanguinalis \\
\hline
\end{tabular}


Plants (continued)

\begin{tabular}{|c|c|}
\hline Common name & Scientific name \\
\hline Late/giant goldenrod & Solidago gigantea \\
\hline Leafy spurge & Euphorbia esula \\
\hline Lobelia spp. & Lobelia spp. \\
\hline Low cudweed & Gnaphalium uliginosum \\
\hline Marsh bedstraw & Galium palustre \\
\hline Marshpepper smartweed & Polygonum hydropiper \\
\hline Meadow goat's beard & Tragopogon pratensis \\
\hline Mentha spp. & Mentha spp. \\
\hline Mouse-ear chickweed & Cerastium vulgatum \\
\hline Mouse-eared hawkweed & Hieracium pilosella \\
\hline Mugwort & Artemisia vulgaris \\
\hline Narrow-leaved goldendrod & Euthamia graminifolia \\
\hline New England aster & Aster novae-angliae \\
\hline New York aster & Symphyotrichum novi-belgii \\
\hline Night-flowering catchfly & Silene noctiflora \\
\hline Nodding thistle & Carduus natans \\
\hline Northern marsh yellow cress & Rorippa palustris \\
\hline Oak-leaved goosefoot & Chenopodium glaucum \\
\hline Orchard grass & Dactylis glomerata \\
\hline Ox-eye daisy & Chrysanthemum leucanthemum \\
\hline Panicled aster & Symphyotrichum lanceolatum \\
\hline Perennial rye grass & Lolium perenne \\
\hline Perennial sow-thistle & Sonchus arvensis \\
\hline Philadelphia fleabane & Erigeron philadelphicus \\
\hline Pigweed spp. & Amaranthaceae \\
\hline Pineappleweed & Matricaria matricarioides \\
\hline Plantain & Plantago major \\
\hline Poison ivy & Rhus radicans \\
\hline Polygonum spp. & Polygonum spp. \\
\hline Prickly lettuce & Lactuca scariola \\
\hline Proso millet & Panicum milliacium \\
\hline Prostrate knotweed & Polygonum aviculare \\
\hline Purple loosestrife & Lythrum salcaria \\
\hline Purslane & Portulaca oleracea \\
\hline Purslane speedwell & Veronica perlgrina \\
\hline Quack grass & Agropyron repens \\
\hline Ragweed & Ambrosia artemisiifolia \\
\hline Raspberry & Rubus idaeus \\
\hline Red clover & Trifolium pratense \\
\hline Red fescue & Festuca rubra \\
\hline Redroot pigweed & Amaranthus retroflexus \\
\hline Redtop & Agrostis gigantea \\
\hline Reed canary grass & Phalaris arundinaceae \\
\hline Rough bedstraw & Galium asprellum \\
\hline Rough cinquefoil & Potentilla norvegica \\
\hline Rough fleabane & Erigeron strigosus \\
\hline Rough-stemmed goldenrod & Solidago rugosa \\
\hline Rubia peregrina & Rubia peregrina \\
\hline Sedge spp. & Carex spp. \\
\hline Sensitive fern & Onoclea sensibilis \\
\hline Shepherd's purse & Capsella bursa-pastoris \\
\hline Silvery cinquefoil & Potentilla argentea \\
\hline Slender rush & Juncus tenuis \\
\hline Small sundrops & Oenothera perennis \\
\hline Smartweed spp. & Polygonaceae spp. \\
\hline Smooth bedstraw/wild madder & Galium mollugo \\
\hline Smooth brome & Bromus inermis \\
\hline Smooth crab grass & Digitaria ischaemum \\
\hline Smoothish hawkweed & Hieracium floribundum \\
\hline Soft rush & Juncus effusus \\
\hline Spikerush spp. & Eleocharis spp. \\
\hline Spiny annual sow-thistle & Sonchus asper \\
\hline
\end{tabular}


Plants (continued)

\begin{tabular}{|c|c|}
\hline Common name & Scientific name \\
\hline Spotted joe-pye weed & Eupatorium maculatum \\
\hline Spreading atriplex & Atriplex patula \\
\hline St. john's-wort & Hypericum perforatum \\
\hline Stiff gentian/agueweed & Gentianella quinquefolia \\
\hline Stinging nettle & Urtica dioica \\
\hline Stinking mayweed & Anthemis cotula \\
\hline Stinkweed/field penny-cress & Thlaspi arvense \\
\hline Strawberry & Fragaria spp. \\
\hline Sulphur cinquefoil & Potentilla recta \\
\hline Swamp milkweed & Asclepias incarnata \\
\hline Sweet clover spp. & Melilotus spp. \\
\hline Tall buttercup & Ranunculus acris \\
\hline Tansy & Tanacetum vulgar \\
\hline Three-seeded mercury & Acalypha rhombides \\
\hline Thyme-leaved speedwell & Veronica serpylliufolia \\
\hline Thyme-leaved spurge & Euphorbia serpyllifolia \\
\hline Timothy & Phleum pratense \\
\hline Toadflax/butter-and-eggs & Linaria vulgaris \\
\hline Trifolium spp. & Trifolium spp. \\
\hline Tufted vetch/purple vetch & Vicia cracca \\
\hline Velvetleaf & Abutilon theophrasti \\
\hline Virginia creeper & Parthenocissus quinquefolia \\
\hline Vitis spp. & Vitis spp. \\
\hline Water horehound & Lycopus americanus \\
\hline White campion/white cockle & Silene latifolia \\
\hline White clover & Trifolium repens \\
\hline White sweet clover & Melilotus officinalis alba \\
\hline Wild buckwheat & Polygonum convolvulus \\
\hline Wild carrot/queen anne's lace & Daucus carota \\
\hline Wild cucumber & Echinocystis lobata \\
\hline Wild lettuce & Lactuca virosa \\
\hline Wild mint/field mint & Mentha arvensis \\
\hline Wild mustard & Sinapis arvensis \\
\hline Wild parsnip & Pastinaca sativa \\
\hline Wild radish & Raphanus raphanistrum \\
\hline Witch grass & Panicum capillare \\
\hline Wormseed mustard & Erysimum cheiranthoides \\
\hline Yarrow & Achillea millefolium \\
\hline Yellow avens & Geum aleppicum \\
\hline Yellow foxtail & Setaria glauca \\
\hline Yellow hawkweed & Hieracium caespitosum \\
\hline Yellow nut sedge & Cyperus esculentus \\
\hline Yellow rocket & Barbarea vulgaris \\
\hline Yellow sweet clover & Melilotus officinalis \\
\hline
\end{tabular}

\section{Butterflies}

\begin{tabular}{ll}
\hline \hline Common name & Scientific name \\
\hline American Lady & Vanessa virginiensis \\
Black Swallowtail & Papilio polyxenes \\
Blue spp. & Polyommatinae spp. \\
Bronze copper & Lycaena hyllus \\
Cabbage white & Pieris rapae \\
Clouded sulphur & Colias philodice \\
Common branded skipper & Hesperia comma laurentina \\
Common ringlet & Coenonympha tullia \\
Common wood-nymph & Cercyonis pegala \\
Eastern tailed blue & Everes comyntas \\
European skipper & Thymelicus lineola \\
Eyed brown & Satyrodes eurydice \\
Great spangled fritillary & Speyeria cybele
\end{tabular}


Lady spp.

Least skipper

Meadow fritillary

Monarch

Mustard white

Northern crescent

Orange sulphur

Painted lady

Pearl crescent

Pecks skipper

Pink-edged sulphur

Question mark

Red admiral

Skipper spp.

Spring azure

Viceroy

White admiral

White spp.
Vanessa spp.

Ancyloxypha numitor

Boloria bellona

Danaus plexippus

Pieris oleracea

Phyciodes cocyta

Colias eurytheme

Vanessa cardui

Phyciodes tharos

Polites peckius

Colias interior

Polygonia interrogationis

Vanessa atalanta

Hesperiidae spp.

Celastrina ladon

Limenitis archippus

Limenitis arthemis

Pieridae spp.

Bees

Scientific name

Agapostemon spp.

Andrena asteris

Andrena chromotricha

Andrena commoda

Andrena hitrcincta

Andrena nasonii

Andrena spp.

Andrena wilkella

Apis mellifera

Augochlora spp.

Augochlorella spp.

Bombus bimaculatus

Bombus borealis

Bombus fervidus

Bombus grisecollis

Bombus impatiens

Bombus rufocinctus

Bombus sandersoni

Bombus ternarius

Bombus terricola

Bombus vagans

Chelostoma rapunculi

Chelostoma spp.

Coelioxys spp.

Diadasia spp.

Dialictus spp.

Evylaeus spp.

Halictus confusus

Halictus ligatus

Halictus rubicundus

Halictus spp.

Hoplitis spp.

Hylaeus affinis

Hylaeus annulatus

Hylaeus modestus

Lasioglossum cinctipes

Lasioglossum coriaceum

Lasioglossum leucozonium

Lasioglossum spp.

Lasioglossum truncatum

Lasioglossum zonulum

Megachile brevis 


\section{Bees (continued)}

Scientific name

Megachile latimanus

Megachile spp.

Melissodes desponsa

Melissodes druriella

Melissodes illata

Melissodes spp.

Melissodes subillata

Melissodes trinodis

Peponapis pruinosa

Sphecodes cressonii

\section{Syrphids}

Scientific name

Allograpta obliqua

Eristalis arbustorum

Eristalis dimidiata

Eristalis tenax

Eupeodes americanus

Eupeodes americanus/pomus

Helophilus fasciatus

Lejops lineatus

Melanostoma mellinum

Paragus spp.

Parhelophilus laetus

Platycheirus hyperboreus

Platycheirus quadratus

Sphaerophoria abbreviata/asymmetrica/philanthus

Sphaerophoria brevipilosa

Sphaerophoria contigua

Sphaerophoria philanthus

Syritta pipiens

Syrphus rectus

Syrphus ribesii

Syrphus vitripennis/rectus

Toxomerus geminatus

Toxomerus marginatus

\section{Carabids}

Scientific name

Agonum cupripenne

Agonum gratiosum

Agonum melanarium

Agonum muelleri

Agonum placidum

Agonum thoreyi

Amara aenea

Amara angustatoides

Amara apricaria

Amara lunicollis

Amara otiosa

Amara pallipes

Amara patruelis

Amara rubrica

Anisodactylus rusticus

Anisodactylus sanctaecrucis

Bembidion frontale

Bembidion inaequale

Bembidion mimus

Bembidion obtusa 


\section{Carabids (continued)}

Scientific name

Bembidion obtusum

Bembidion quadrimaculatum

Biemus discus

Bradycellus nigriceps

Carabus granulatus

Carabus nemoralis

Chlaenius pusillus

Chlaenius sericeus

Chlaenius tricolor

Cicindela punctualata

Cicindela sexguttata

Clivina fossor

Diplocheila obtusa

Dyschirius montanus

Elaphropus incurvus

Harpalus affinis

Harpalus caliginosus

Harpalus erraticus

Harpalus erythropus

Harpalus herbivagus

Harpalus pensylvanicus

Harpalus pusillus

Harpalus rubripes

Harpalus rufipes

Notiobia terminata

Notiophilus semistriatus

Paratychus proximus

Patrobus longicornis

Poecilus chalcites

Poecilus lepidus

Poecilus lucublandus

Pterostichus caudicalis

Pterostichus commutabilis

Pterostichus melanarius

Pterostichus patruelis

Stenolophus comma

Xestonotus lugubris

\section{Spiders}

Scientific name

Agelenopsis potteri

Agelenopsis spp.

Agyneta fabra

Agyneta spp.

Agyneta unimaculata

Allomengea dentisetis

Araneidae spp.

Arctosa emertoni

Arctosa spp.

Argenna obesa

Bathyphantes concolor

Bathyphantes pallidus

Castianeira descripta

Clubiona abbotii

Clubiona johnsoni

Coriarachne spp.

Dictyna foliacea

Erigone atra

Euryopis funebris

Glenognatha spp.

Gnaphosa parvula 


\section{Spider (continued)}

Scientific name

Gnaphosa spp.

Grammonota gentilis

Halorates plumosus

Islandiana longisetosa

Linyphiidae spp.

Lycosidae spp.

Micaria pulicaria

Microlinyphia pusilla

Misumenops spp.

Neoantistea agilis

Neoantistea spp.

Ozyptila georgiana

Pachygnatha spp.

Pachygnatha tristriata

Pachygnatha xanthostoma

Pardosa distincta

Pardosa fuscula

Pardosa milvina

Castianeira longipalpa

Pardosa modica

Pardosa moesta

Pardosa saxatilis

Pardosa spp.

Philodromus cespitum

Pirata aspirans

Pirata insularis

Pirata minutus

Pirata sedentarius

Pirata spp.

Pirata zelotes

Salticidae spp.

Steatoda americana

Talavera minuta

Tennesseellum formicum

Tetragnatha extensa

Tetragnathidae spp.

Thomisidae spp.

Tibellus oblongus

Trochosa ruricola

Trochosa spp.

Walckenaeria fallax

Walckenaeria spp.

Walckenaeria spiralis

Xysticus discursans

Xysticus ferox

Xysticus fervidus

Zelotes fratris

Zelotes spp. 


\section{Appendix II. Remote sensing data pre-processing. MODIS}

\section{Downloading MODIS Data}

The MOD09GQ Surface Reflectance (Daily higher-order gridded level-2 (L2G) Global 250m), the MOD09GA Surface Reflectance (Daily L2G Global $1 \mathrm{~km}$ and 500m) the MOD10A1 Snow Cover (Daily level-3 (L3) Global 500m Grid) data products, each archived as tiles in HDF-EOS or Hierarchical Data Format (*.hdf) are required for the full operational implementation of CALMS as input. The CALMS downloads MOD09GA and MOD09GQ data via http from the USGS Earth Resources Observation and Science (EROS) Data Center archives, and MOD10A1data via ftp from the US National Snow and Ice Data Center.

\section{Science Dataset Extraction}

The MODIS Science Datasets (SDS) required to implement CALMS over any 7-day period are extracted, each in turn, from the HDF-EOS tiles downloaded from that period. To do this, CALMS uses the MODIS Reprojection Tool (MRT), a tool provided free of charge by the USGS. CALMS uses the MRT to read MODIS Level-2G HDF-EOS data files, select the subset of SDS to be extracted, and to extract the SDS and save them in the GeoTIFF file format.

\section{Checking for and Filling Missing Data}

CALMS then checks the data to determine if there are any missing HDF-EOS tiles for a given 7day compositing period. If no data are missing, it continues data processing. However, if data are missing, CALMS either reports the missing data and exits or fills the data gaps and continues with data processing (repair mode). Filling gaps involves identifying the GeoTIFF files expected for an n-day, n-tile composite (usually 7- day, 12-tiled) and identifying if any of the expected tiles are missing, and finally using the available tiles to fill gaps in the data (missing hdf files for a 
given day are gap-filled using the hdf files extracted for the previous or subsequent day, Davidson, 2015).

\section{Daily Quality Control, Cloud and Snow Mosaicking}

CALMS then takes all of the individual MOD10A1 SDS for the 7-day compositing period that were extracted in the previous step and mosaics them to create seven daily quality control, cloud and snow composites. The pixel screening criteria for quality control and cloud and snow composites are listed below:
Cloud state $=$ clear
Bitmap Value $==0$
Cloud shadow $=$ no
Bitmap Value $==0$
Aerosol quantity $=$ not high
Bitmap Value $<>3$
Cirrus quantity $=$ not high
Bitmap Value $<>3$
Sensor zenith between 0 and 35 degrees
Bitmap Value $>=0$ and $<=3500$

\section{Weekly Maximum-NDVI Compositing (All Quality)}

CALMS extracts the MOD09 SDS for the 7-day compositing period and creates a weekly "All quality" Maximum-NDVI composite and its associated 'day-of-week of Maximum-NDVI' composite. Furthermore, the Max-NDVI compositor eliminates those pixels of poor quality from the compositing process. Such pixels are identified as those whose reflectances in the R and NIR bands have been retrieved under partially or fully cloudy conditions, contain cloud shadow, high quantities of aerosols or cirrus clouds, and those whose observations were collected at sensor zenith angles greater than 35 degrees.

\section{Day-Of-Week Quality Control, Cloud and Snow Compositing}

CALMS then assigns quality control, cloud and snow information to the "All-Quality" MaxNDVI product. To do this, it cross-references the day-of-week Max-NDVI composite and each daily quality control, cloud and snow mosaic to create a single day-of-week quality control, cloud and snow composite. The pixel values in each of these daily composites correspond to the quality 
control, cloud cover and snow fraction information for each Max-NDVI pixel in the "All-quality" Maximum-NDVI product.

\section{Weekly Maximum-NDVI Compositing (Best Quality)}

In the next step, the weekly "All quality" Maximum-NDVI composite and its associated quality control, cloud and snow mosaic are cross-referenced by CALMS to create a weekly "Best quality" Maximum-NDVI composite for snow-free surfaces. To implement this step, pixel values in the “All-Quality" product retrieved under poor atmospheric conditions and over snow-covered surfaces are removed. In addition, negative Max-NDVI values in the "All-Quality" product, occurring where $\mathrm{R}$ reflectance is larger than NIR reflectance, are considered non-vegetated and re-set to a value of 0 . This process creates a higher-quality ("Best Quality") Max-NDVI product whose values span a biomass (NDVI) gradient ranging from 0 (no/low biomass) to 1 (high biomass).

\section{Historical (Best Quality) Mean (Baseline) NDVI Calculation}

In order to calculate weekly anomalies (departures from normal conditions), CALMS uses the AAFC historical archive of 7-day "Best Quality" weekly NDVI observations to create mean ("baseline" or "normal") weekly NDVI composites. It uses the selected files to create two data products. In the first product, each pixel value corresponds to the mean historical "Best-quality" Max-NDVI value for a given week, as calculated from all previous years in the MODIS historical record (i.e. does not include data from the current year). In the second product, each pixel value corresponds to the number (count) of valid "Best-quality" Max-NDVI values used to calculate the mean weekly values for that pixel. 


\section{Weekly (Best Quality) Difference from Mean (Anomaly) NDVI Calculation}

To compare and provide an insight into weekly departures from normal growing conditions, CALMS calculates the difference between each weekly "All quality" Maximum-NDVI composite and its associated historical 7-day "Best Quality" Maximum-NDVI baseline (mean), i.e. "anomalies". These differences indicate whether the current weekly Max-NDVI is normal, or above or below normal for that particular week. Anomalies are calculated by subtracting the historical Max-NDVI baseline dataset from the weekly “All quality” Maximum-NDVI composite and range between -1 and 1 . Anomaly values greater than 0 indicate locations which are greener than the historic mean for that week, while values less than 0 indicate locations which are less green than the historic mean for that week.

\section{Subsetting (Clipping)}

CALMS clips the various output products produced during its processing to any geographical extent of interest. CALMS data products are generally clipped to the Canadian national extent and to the extent of Canada's agricultural regions.

\section{Landsat}

LEDAPS inputs water vapour, ozone, geopotential height (height of a pressure surface above mean sea level), aerosol optical thickness, and a digital elevation model along with the Landsat data and uses the Second Simulation of a Satellite Signal in the Solar Spectrum (6S) radiative transfer model (Vermote et al., 1997) to generate top of atmosphere (TOA) reflectance, surface reflectance, and clouds, cloud shadows, adjacent clouds, land, and water masks. The result is delivered as the Landsat Surface Reflectance data product. TOA reflectance data are generated by applying calibration equations to Landsat digital numbers using per-pixel solar illumination angles derived from band 4 (closest to centre of the focal plane). The surface reflectance data 
products are derived from TOA data using the $6 \mathrm{~S}$ atmospheric correction model. Landsat surface reflectance outputs for Landsat 5 TM include Surface Reflectance data files (Bands 1-5, 7), Surface Reflectance quality files, Surface Reflectance and Level-1 metadata file (.xml) and Quality Assessment bands. Quality Assessment bands include: 1) the sr_cloud_qa band, which identifies pixels containing dark dense vegetation, cloud, cloud shadow, snow, land/water and adjacent cloud, 2) the sr_atmos_opacity band, which estimates the atmospheric contamination in each pixel, 3) the pixel_qa band, which is an alternative quality assessment algorithm for clouds, cloud shadow, snow, ice and water and is likely to present a more accurate result than the LEDAPS sr_cloud_qa band (LEDAPS Product Guide, USGS, 2018), and 4) the radsat_qa band, which indicates any bands that were saturated during data capture yielding unusable data. The Landsat surface reflectance data product is generated with $30 \mathrm{~m}$ nominal pixel sample size on a Universal Transverse Mercator (UTM) projection and the default file format is GeoTIFF. 
Appendix III. Correlation matrices of MODIS 2011 and 2012 explanatory variables for 3 $\times 3 \mathbf{~ k m}$ sample landscapes. 


\begin{tabular}{|c|c|c|c|c|c|c|c|c|c|c|c|c|c|c|c|c|c|c|c|c|c|c|}
\hline 2011 & 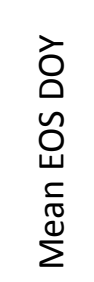 & 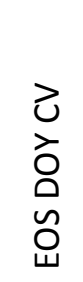 & 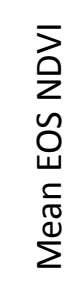 & 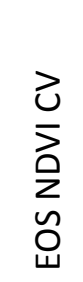 & 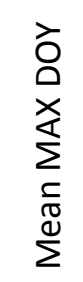 & 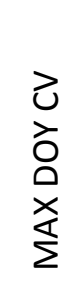 & 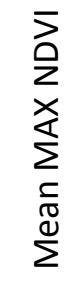 & 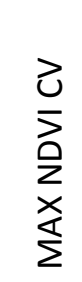 & 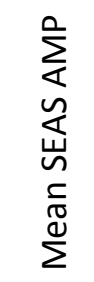 & 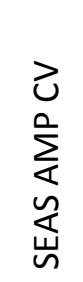 & 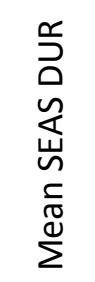 & 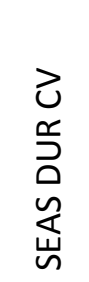 & 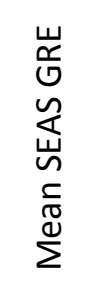 & 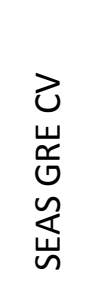 & 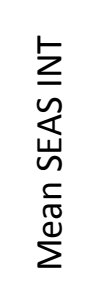 & 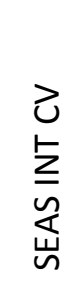 & 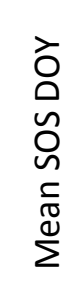 & 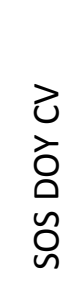 & 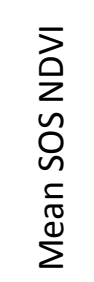 & 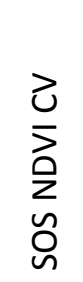 & 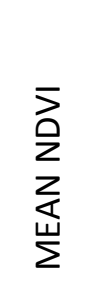 & $\begin{array}{l}\sum \\
\text { ¿ } \\
\text { 己 }\end{array}$ \\
\hline MEAN EOS DOY & 1 & & & & & & & & & & & & & & & & & & & & & \\
\hline EOS DOY CV & 0.32 & 1 & & & & & & & & & & & & & & & & & & & & \\
\hline MEAN EOS NDVI & 0.54 & -0.26 & 1 & & & & & & & & & & & & & & & & & & & \\
\hline EOS NDVI CV & 0.07 & 0.69 & -0.48 & 1 & & & & & & & & & & & & & & & & & & \\
\hline MEAN MAX DOY & 0.75 & 0.58 & 0.09 & 0.38 & 1 & & & & & & & & & & & & & & & & & \\
\hline MAX DOY CV & 0.57 & 0.59 & 0.23 & 0.26 & 0.53 & 1 & & & & & & & & & & & & & & & & \\
\hline MEAN MAX NDVI & -0.43 & -0.47 & 0.34 & -0.43 & -0.51 & -0.36 & 1 & & & & & & & & & & & & & & & \\
\hline MAX NDVI CV & 0.06 & 0.21 & -0.22 & 0.43 & 0.10 & 0.29 & -0.40 & 1 & & & & & & & & & & & & & & \\
\hline MEAN SEAS AMP & -0.33 & 0.23 & -0.66 & 0.37 & 0.17 & -0.21 & 0.19 & -0.07 & 1 & & & & & & & & & & & & & \\
\hline SEAS AMP CV & 0.14 & 0.34 & -0.15 & 0.45 & 0.16 & 0.19 & -0.47 & 0.38 & -0.21 & 1 & & & & & & & & & & & & \\
\hline MEAN SEAS DUR & 0.23 & -0.40 & 0.81 & -0.54 & -0.31 & 0.09 & 0.16 & -0.07 & -0.72 & -0.02 & 1 & & & & & & & & & & & \\
\hline SEAS DUR CV & -0.08 & 0.56 & -0.67 & 0.72 & 0.37 & 0.05 & -0.30 & 0.18 & 0.65 & 0.46 & -0.83 & 1 & & & & & & & & & & \\
\hline MEAN SEAS GRE & -0.19 & 0.10 & -0.34 & 0.18 & 0.19 & -0.18 & 0.41 & -0.18 & 0.71 & -0.42 & -0.69 & 0.39 & 1 & & & & & & & & & \\
\hline SEAS GRE CV & 0.14 & 0.11 & 0.12 & 0.17 & -0.06 & 0.25 & -0.37 & 0.48 & -0.54 & 0.87 & 0.35 & 0.02 & -0.67 & 1 & & & & & & & & \\
\hline MEAN SEAS INT & 0.01 & -0.54 & 0.84 & -0.64 & -0.46 & -0.09 & 0.62 & -0.26 & -0.64 & -0.24 & 0.77 & -0.79 & -0.35 & 0.10 & 1 & & & & & & & \\
\hline SEAS INT CV & -0.05 & 0.43 & -0.51 & 0.72 & 0.27 & 0.23 & -0.26 & 0.56 & 0.46 & 0.32 & -0.59 & 0.74 & 0.29 & 0.06 & -0.59 & 1 & & & & & & \\
\hline MEAN SOS DOY & 0.16 & 0.54 & -0.61 & 0.57 & 0.65 & 0.13 & -0.33 & 0.10 & 0.70 & 0.08 & -0.72 & 0.71 & 0.62 & -0.30 & -0.78 & 0.58 & 1 & & & & & \\
\hline SOS DOY CV & -0.10 & 0.41 & -0.61 & 0.65 & 0.29 & 0.18 & -0.26 & 0.28 & 0.56 & 0.49 & -0.73 & 0.72 & 0.31 & 0.11 & -0.70 & 0.69 & 0.70 & 1 & & & & \\
\hline MEAN SOS NDVI & -0.02 & -0.53 & 0.81 & -0.62 & -0.50 & -0.08 & 0.54 & -0.22 & -0.72 & -0.15 & 0.74 & -0.77 & -0.49 & 0.20 & 0.78 & -0.58 & -0.72 & -0.67 & 1 & & & \\
\hline SOS NDVI CV & 0.05 & 0.40 & -0.33 & 0.75 & 0.28 & 0.29 & -0.25 & 0.35 & 0.28 & 0.45 & -0.40 & 0.67 & 0.15 & 0.19 & -0.43 & 0.70 & 0.43 & 0.75 & -0.42 & 1 & & \\
\hline Mean NDVI & -0.01 & -0.50 & 0.81 & -0.60 & -0.38 & -0.14 & 0.78 & -0.36 & -0.39 & -0.36 & 0.70 & -0.69 & -0.07 & -0.09 & 0.85 & -0.53 & -0.71 & -0.63 & 0.74 & -0.40 & 1 & \\
\hline CV NDVI & 0.03 & 0.39 & -0.38 & 0.70 & 0.21 & 0.36 & -0.40 & 0.77 & 0.17 & 0.34 & -0.32 & 0.45 & 0.03 & 0.28 & -0.46 & 0.74 & 0.34 & 0.54 & -0.43 & 0.66 & -0.50 & 1 \\
\hline
\end{tabular}




\begin{tabular}{|c|c|c|c|c|c|c|c|c|c|c|c|c|c|c|c|c|c|c|c|c|c|c|}
\hline 2012 & 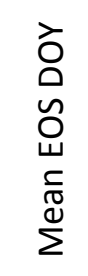 & 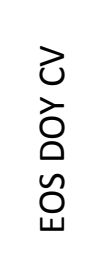 & 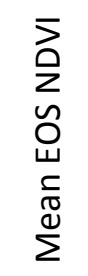 & $\begin{array}{l}己 \\
\text { 己 } \\
\text { 之 } \\
\text { ○ิ }\end{array}$ & 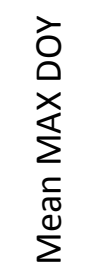 & 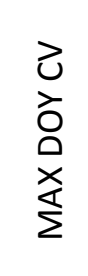 & 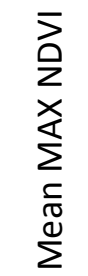 & 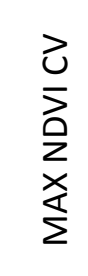 & 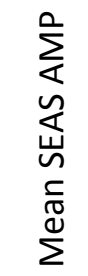 & 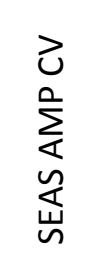 & 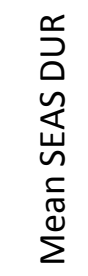 & 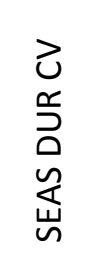 & 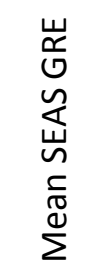 & 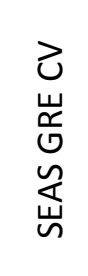 & 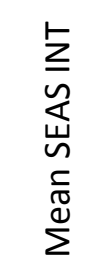 & 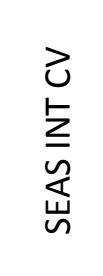 & 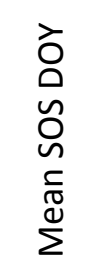 & $\begin{array}{l}\text { 己े } \\
\text { ठ̀ } \\
\text { ஸे } \\
\text { ○ิ }\end{array}$ & 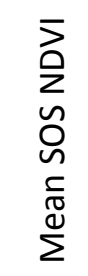 & $\begin{array}{l}\text { 己 } \\
\sum_{2} \\
\sum_{n} \\
\text { O }\end{array}$ & 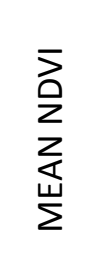 & $\begin{array}{l}\text { 方 } \\
\text { 己 }\end{array}$ \\
\hline MEAN EOS DOY & 1 & & & & & & & & & & & & & & & & & & & & & \\
\hline EOS DOY CV & 0.34 & 1 & & & & & & & & & & & & & & & & & & & & \\
\hline MEAN EOS NDVI & 0.77 & -0.10 & 1 & & & & & & & & & & & & & & & & & & & \\
\hline EOS NDVI CV & -0.40 & 0.33 & -0.61 & 1 & & & & & & & & & & & & & & & & & & \\
\hline MEAN MAX DOY & 0.77 & 0.59 & 0.30 & -0.15 & 1 & & & & & & & & & & & & & & & & & \\
\hline MAX DOY CV & 0.66 & 0.70 & 0.29 & 0.01 & 0.70 & 1 & & & & & & & & & & & & & & & & \\
\hline MEAN MAX NDVI & -0.21 & -0.58 & 0.30 & -0.38 & -0.26 & -0.48 & 1 & & & & & & & & & & & & & & & \\
\hline MAX NDVI CV & -0.07 & 0.06 & -0.13 & 0.38 & -0.14 & 0.10 & -0.23 & 1 & & & & & & & & & & & & & & \\
\hline MEAN SEAS AMP & -0.66 & -0.22 & -0.69 & 0.31 & -0.14 & -0.50 & 0.37 & -0.09 & 1 & & & & & & & & & & & & & \\
\hline SEAS AMP CV & 0.28 & 0.38 & 0.14 & 0.15 & 0.12 & 0.40 & -0.45 & 0.31 & -0.50 & 1 & & & & & & & & & & & & \\
\hline MEAN SEAS DUR & 0.71 & 0.05 & 0.86 & -0.47 & 0.14 & 0.40 & -0.10 & 0.02 & -0.84 & 0.32 & 1 & & & & & & & & & & & \\
\hline SEAS DUR CV & -0.18 & 0.57 & -0.55 & 0.45 & 0.39 & 0.30 & -0.23 & 0.02 & 0.46 & 0.25 & -0.61 & 1 & & & & & & & & & & \\
\hline MEAN SEAS GRE & -0.55 & -0.36 & -0.52 & 0.19 & -0.13 & -0.57 & 0.49 & -0.13 & 0.76 & -0.59 & -0.73 & 0.26 & 1 & & & & & & & & & \\
\hline SEAS GRE CV & 0.39 & 0.33 & 0.31 & 0.00 & 0.10 & 0.46 & -0.43 & 0.32 & -0.67 & 0.76 & 0.52 & 0.05 & -0.74 & 1 & & & & & & & & \\
\hline MEAN SEAS INT & 0.48 & -0.33 & 0.72 & -0.61 & -0.07 & 0.02 & 0.50 & -0.11 & -0.61 & 0.03 & 0.81 & -0.69 & -0.43 & 0.21 & 1 & & & & & & & \\
\hline SEAS INT CV & -0.40 & 0.05 & -0.51 & 0.81 & -0.13 & -0.03 & -0.09 & 0.50 & 0.41 & -0.05 & -0.49 & 0.43 & 0.32 & -0.15 & -0.47 & 1 & & & & & & \\
\hline MEAN SOS DOY & -0.28 & 0.24 & -0.65 & 0.35 & 0.38 & -0.02 & -0.02 & -0.09 & 0.71 & -0.24 & -0.76 & 0.77 & 0.71 & -0.43 & -0.78 & 0.40 & 1 & & & & & \\
\hline SOS DOY CV & -0.08 & 0.45 & -0.43 & 0.39 & 0.40 & 0.36 & -0.19 & 0.06 & 0.38 & 0.30 & -0.51 & 0.94 & 0.21 & 0.14 & -0.58 & 0.49 & 0.69 & 1 & & & & \\
\hline MEAN SOS NDVI & 0.53 & -0.17 & 0.71 & -0.57 & -0.03 & 0.19 & 0.31 & -0.07 & -0.77 & 0.19 & 0.73 & -0.62 & -0.65 & 0.39 & 0.76 & -0.48 & -0.74 & -0.51 & 1 & & & \\
\hline SOS NDVI CV & -0.39 & 0.00 & -0.50 & 0.69 & -0.17 & -0.11 & -0.14 & 0.27 & 0.38 & 0.16 & -0.48 & 0.46 & 0.29 & 0.01 & -0.48 & 0.78 & 0.37 & 0.54 & -0.48 & 1 & & \\
\hline Mean NDVI & -0.13 & 0.01 & -0.23 & 0.08 & -0.08 & -0.17 & -0.20 & 0.10 & 0.05 & 0.12 & -0.12 & 0.10 & 0.02 & 0.05 & -0.22 & -0.02 & 0.06 & 0.02 & -0.20 & 0.00 & 1 & \\
\hline CV NDVI & -0.03 & -0.10 & -0.02 & -0.01 & 0.00 & 0.02 & 0.10 & 0.16 & 0.12 & -0.11 & -0.10 & 0.05 & 0.12 & -0.12 & -0.02 & 0.16 & 0.10 & 0.11 & -0.05 & 0.06 & -0.36 & 1 \\
\hline
\end{tabular}


Appendix IV: RFR mean square residuals and percent variance accounted for: MODIS and Landsat data, $1 \times 1 \mathrm{~km}$ and $3 \times 3 \mathrm{~km}$ sample landscapes for each taxon and each year independently. 


\begin{tabular}{|c|c|c|c|c|c|c|c|c|c|c|c|c|c|c|}
\hline \multicolumn{2}{|c|}{$1 \mathrm{~km}-\mathrm{MODIS}$} & 2000 & 2001 & 2002 & 2003 & 2004 & 2005 & 2006 & 2007 & 2008 & 2009 & 2010 & 2011 & 2012 \\
\hline \multicolumn{15}{|c|}{ Plants } \\
\hline \multirow{3}{*}{ MSR } & Mean & 246.29 & 250.54 & 247.77 & 241.30 & 234.67 & 250.69 & 261.45 & 259.64 & 280.28 & 8.53 & 221.98 & 239.97 & 219.78 \\
\hline & $\max$ & 252.41 & 255.22 & 253.89 & 246.90 & 240.04 & 256.51 & 266.94 & 265.42 & 287.48 & 8.74 & 225.96 & 246.99 & 225.74 \\
\hline & $\min$ & 239.77 & 244.52 & 241.12 & 235.87 & 229.33 & 245.37 & 257.04 & 254.50 & 273.79 & 8.32 & 217.62 & 233.26 & 215.28 \\
\hline \multirow{3}{*}{$\% \operatorname{Var}$} & Mean & -7.79 & -9.64 & -8.43 & -5.60 & -2.70 & -9.71 & -14.42 & -13.63 & -22.66 & 2.38 & 2.85 & -5.02 & 3.82 \\
\hline & $\max$ & -4.93 & -7.01 & -5.52 & -3.22 & -0.36 & -7.38 & -12.49 & -11.38 & -19.82 & 4.85 & 4.76 & -2.08 & 5.79 \\
\hline & $\min$ & -10.47 & -11.69 & -11.11 & -8.05 & -5.05 & -12.26 & -16.82 & -16.16 & -25.81 & 0.06 & 1.11 & -8.09 & 1.21 \\
\hline \multicolumn{15}{|c|}{ Beetles } \\
\hline \multirow{3}{*}{ MSR } & Mean & 14.79 & 13.43 & 15.95 & 15.12 & 16.51 & 15.74 & 14.28 & 14.62 & 12.83 & 8.49 & 13.06 & 14.64 & 16.63 \\
\hline & $\max$ & 15.16 & 13.74 & 16.42 & 15.43 & 16.85 & 16.11 & 14.71 & 14.97 & 13.10 & 8.67 & 13.44 & 14.93 & 17.19 \\
\hline & $\min$ & 14.50 & 13.13 & 15.61 & 14.76 & 16.18 & 15.36 & 13.93 & 14.31 & 12.51 & 8.28 & 12.78 & 14.27 & 16.27 \\
\hline \multirow{3}{*}{$\% \operatorname{Var}$} & Mean & -7.62 & 2.27 & -16.08 & -9.98 & -20.13 & -14.51 & -3.87 & -6.39 & 6.63 & -27.32 & 4.97 & -6.49 & -20.98 \\
\hline & $\max$ & -5.46 & 4.48 & -13.58 & -7.4 & -17.74 & -11.75 & -1.35 & -4.14 & 8.98 & -24.13 & 7.02 & -3.83 & -18.37 \\
\hline & $\min$ & -10.32 & 0.02 & -19.44 & -12.28 & -22.61 & -17.21 & -7 & -8.91 & 4.72 & -30.07 & 2.2 & -8.66 & -25.07 \\
\hline \multicolumn{15}{|c|}{ Birds } \\
\hline \multirow{3}{*}{ MSR } & Mean & 8.10 & 7.71 & 7.61 & 8.35 & 7.66 & 7.90 & 8.33 & 8.29 & 7.77 & 0.00 & 7.34 & 7.58 & 7.00 \\
\hline & $\max$ & 8.27 & 7.88 & 7.79 & 8.56 & 7.83 & 8.08 & 8.51 & 8.48 & 7.94 & 0.00 & 7.55 & 7.79 & 7.17 \\
\hline & $\min$ & 7.92 & 7.50 & 7.43 & 8.15 & 7.40 & 7.71 & 8.16 & 8.12 & 7.57 & 0.00 & 7.08 & 7.44 & 6.81 \\
\hline \multirow{3}{*}{$\% \operatorname{Var}$} & Mean & -21.53 & -15.63 & -14.11 & -25.23 & -14.89 & -18.50 & -24.94 & -24.27 & -16.52 & -10.52 & -10.13 & -13.68 & -5.05 \\
\hline & $\max$ & -18.83 & -12.44 & -11.4 & -22.21 & -10.94 & -15.57 & -22.46 & -21.74 & -13.49 & -7.62 & -6.21 & -11.63 & -2.1 \\
\hline & $\min$ & -24.05 & -18.16 & -16.79 & -28.35 & -17.45 & -21.22 & -27.57 & -27.25 & -19.04 & -13.46 & -13.3 & -16.84 & -7.47 \\
\hline \multicolumn{15}{|c|}{ Butterflies } \\
\hline \multirow{3}{*}{ MSR } & Mean & 9.39 & 10.48 & 9.50 & 10.21 & 9.80 & 9.88 & 10.60 & 9.70 & 10.64 & 0.00 & 10.15 & 9.23 & 9.78 \\
\hline & $\max$ & 9.59 & 10.73 & 9.80 & 10.43 & 10.09 & 10.15 & 10.81 & 9.93 & 10.99 & 0.00 & 10.42 & 9.47 & 10.05 \\
\hline & $\min$ & 9.15 & 10.27 & 9.31 & 9.92 & 9.59 & 9.64 & 10.27 & 9.45 & 10.33 & 0.00 & 9.95 & 9.05 & 9.51 \\
\hline \multirow{3}{*}{$\%$ Var } & Mean & -7.45 & -19.94 & -8.66 & -16.76 & -12.10 & -13.05 & -21.32 & -11.02 & -21.72 & 79.61 & -16.13 & -5.65 & -11.83 \\
\hline & $\max$ & -4.68 & -17.53 & -6.56 & -13.49 & -9.72 & -10.33 & -17.52 & -8.11 & -18.18 & 80.48 & -13.8 & -3.48 & -8.8 \\
\hline & $\min$ & -9.75 & -22.8 & -12.1 & -19.32 & -15.45 & -16.16 & -23.65 & -13.63 & -25.71 & 78.43 & -19.21 & -8.32 & -15.01 \\
\hline
\end{tabular}




\begin{tabular}{|c|c|c|c|c|c|c|c|c|c|c|c|c|c|c|}
\hline \multicolumn{2}{|c|}{$3 \mathrm{~km}$-MODIS } & 2000 & 2001 & 2002 & 2003 & 2004 & 2005 & 2006 & 2007 & 2008 & 2009 & 2010 & 2011 & 2012 \\
\hline \multicolumn{15}{|c|}{ Plants } \\
\hline \multirow{3}{*}{ MSR } & Mean & 180.74 & 188.48 & 167.76 & 170.72 & 186.97 & 188.91 & 201.26 & 174.46 & 166.10 & 216.27 & 172.13 & 186.96 & 178.22 \\
\hline & $\max$ & 184.56 & 193.16 & 173.05 & 176.42 & 193.43 & 193.77 & 207.80 & 178.72 & 169.98 & 221.41 & 176.50 & 191.37 & 184.21 \\
\hline & $\min$ & 177.11 & 180.85 & 162.97 & 165.59 & 183.36 & 184.81 & 196.18 & 170.84 & 162.40 & 211.99 & 168.25 & 181.37 & 174.60 \\
\hline \multirow{3}{*}{$\%$ Var } & Mean & 20.90 & 17.51 & 26.58 & 25.29 & 18.17 & 17.33 & 11.92 & 23.65 & 27.31 & 5.35 & 24.67 & 18.18 & 22.00 \\
\hline & $\max$ & 22.49 & 20.86 & 28.68 & 27.53 & 19.76 & 19.12 & 14.14 & 25.24 & 28.93 & 7.23 & 26.37 & 20.63 & 23.59 \\
\hline & $\min$ & 19.23 & 15.47 & 24.27 & 22.79 & 15.35 & 15.2 & 9.06 & 21.78 & 25.61 & 3.1 & 22.76 & 16.25 & 19.38 \\
\hline \multicolumn{15}{|c|}{ Beetles } \\
\hline \multirow{3}{*}{ MSR } & Mean & 13.66 & 13.46 & 14.45 & 13.56 & 14.82 & 13.06 & 12.53 & 13.96 & 13.31 & 12.56 & 13.55 & 12.35 & 13.31 \\
\hline & $\max$ & 13.96 & 13.86 & 14.81 & 13.94 & 15.19 & 13.38 & 12.86 & 14.25 & 13.62 & 12.83 & 13.84 & 12.67 & 13.70 \\
\hline & $\min$ & 13.41 & 13.18 & 14.09 & 13.20 & 14.51 & 12.78 & 12.19 & 13.73 & 13.00 & 12.15 & 13.23 & 12.09 & 12.99 \\
\hline \multirow{3}{*}{$\%$ Var } & Mean & 0.64 & 2.08 & -5.12 & 1.33 & -7.82 & 4.96 & 8.87 & -1.54 & 3.14 & 8.62 & 1.43 & 10.16 & 3.14 \\
\hline & $\max$ & 2.43 & 4.08 & -2.51 & 3.97 & -5.56 & 7.02 & 11.33 & 0.1 & 5.42 & 11.58 & 3.72 & 12.06 & 5.48 \\
\hline & $\min$ & -1.59 & -0.84 & -7.71 & -1.43 & -10.51 & 2.67 & 6.45 & -3.66 & 0.9 & 6.68 & -0.72 & 7.85 & 0.29 \\
\hline \multicolumn{15}{|c|}{ Birds } \\
\hline \multirow{3}{*}{ MSR } & Mean & 6.99 & 7.56 & 7.86 & 7.54 & 6.83 & 6.70 & 7.02 & 6.85 & 6.50 & 7.83 & 6.71 & 7.03 & 7.31 \\
\hline & $\max$ & 7.20 & 7.77 & 8.06 & 7.72 & 6.97 & 6.86 & 7.22 & 6.99 & 6.65 & 7.97 & 6.86 & 7.20 & 7.50 \\
\hline & $\min$ & 6.81 & 7.42 & 7.73 & 7.39 & 6.64 & 6.60 & 6.84 & 6.74 & 6.38 & 7.64 & 6.49 & 6.87 & 7.17 \\
\hline \multirow{3}{*}{$\% \operatorname{Var}$} & Mean & -4.82 & -13.36 & -13.02 & -17.91 & -2.37 & -0.54 & -5.32 & -2.74 & 2.44 & -17.41 & -0.62 & -5.40 & -9.61 \\
\hline & $\max$ & -2.13 & -11.27 & -10.81 & -15.97 & 0.42 & 1.05 & -2.66 & -1.07 & 4.3 & -14.52 & 2.63 & -2.98 & -7.6 \\
\hline & $\min$ & -7.98 & -16.53 & -15.75 & -20.81 & -4.51 & -2.88 & -8.35 & -4.89 & 0.27 & -19.48 & -2.94 & -8.02 & -12.49 \\
\hline \multicolumn{15}{|c|}{ Butterflies } \\
\hline \multirow{3}{*}{ MSR } & Mean & 7.99 & 8.99 & 8.91 & 9.81 & 7.21 & 8.91 & 8.43 & 9.69 & 10.13 & 9.96 & 8.81 & 9.38 & 8.97 \\
\hline & $\max$ & 8.19 & 9.47 & 9.11 & 10.04 & 7.36 & 9.16 & 8.65 & 9.90 & 10.45 & 10.22 & 9.21 & 9.67 & 9.18 \\
\hline & $\min$ & 7.72 & 8.73 & 8.70 & 9.59 & 7.00 & 8.66 & 8.18 & 9.42 & 9.87 & 9.63 & 8.58 & 9.08 & 8.74 \\
\hline \multirow{3}{*}{$\%$ Var } & Mean & 8.65 & -2.87 & -1.95 & -12.28 & 17.54 & -1.88 & 3.56 & -10.88 & -15.91 & -13.89 & -0.79 & -7.29 & -2.60 \\
\hline & $\max$ & 11.66 & 0.16 & 0.47 & -9.76 & 19.92 & 0.87 & 6.47 & -7.72 & -12.89 & -10.19 & 1.81 & -3.87 & 0.02 \\
\hline & $\min$ & 6.29 & -8.35 & -4.28 & -14.82 & 15.85 & -4.84 & 0.99 & -13.24 & -19.54 & -16.93 & -5.38 & -10.57 & -5.03 \\
\hline
\end{tabular}




\begin{tabular}{|c|c|c|c|c|c|c|c|c|c|c|c|c|c|c|c|c|c|}
\hline \multicolumn{2}{|c|}{$1 \mathrm{~km}$-Landsat } & 1985 & 1987 & 1994 & 1995 & 1996 & 1997 & 1998 & 2001 & 2003 & 2005 & 2006 & 2007 & 2008 & 2009 & 2010 & 2011 \\
\hline \multicolumn{18}{|c|}{ Plants } \\
\hline \multirow[b]{3}{*}{ MSR } & Mean & 248.16 & 204.92 & 238.54 & 223.68 & 242.33 & 234.64 & 253.50 & 235.30 & 270.06 & 222.66 & 279.27 & 250.01 & 238.50 & 252.66 & 258.60 & 239.36 \\
\hline & $\max$ & 255.34 & 210.73 & 243.45 & 228.98 & 248.20 & 240.22 & 260.02 & 240.12 & 274.75 & 227.45 & 285.94 & 255.82 & 243.48 & 257.15 & 264.63 & 246.03 \\
\hline & $\min$ & 243.52 & 199.56 & 234.26 & 218.85 & 236.94 & 229.72 & 246.80 & 231.31 & 264.75 & 218.10 & 272.85 & 241.67 & 234.12 & 247.63 & 253.08 & 233.25 \\
\hline \multirow{3}{*}{$\begin{array}{c}\% \\
\text { Var }\end{array}$} & Mean & -8.60 & 10.32 & -4.39 & 2.11 & -6.05 & -2.69 & -10.94 & -2.97 & -18.19 & 2.56 & -22.22 & -9.41 & -4.37 & -10.57 & -13.17 & -4.75 \\
\hline & $\max$ & -6.57 & 12.66 & -2.52 & 4.22 & -3.69 & -0.53 & -8.01 & -1.23 & -15.86 & 4.55 & -19.41 & -5.76 & -2.46 & -8.37 & -10.76 & -2.08 \\
\hline & $\min$ & -11.75 & 7.78 & -6.54 & -0.21 & -8.62 & -5.13 & -13.80 & -5.09 & -20.24 & 0.46 & -25.14 & -11.96 & -6.56 & -12.54 & -15.81 & -7.67 \\
\hline \multicolumn{18}{|c|}{ Beetles } \\
\hline \multirow[b]{3}{*}{ MSR } & Mean & 16.52 & 13.84 & 13.41 & 14.49 & 15.18 & 15.80 & 13.60 & 14.53 & 15.37 & 15.64 & 15.03 & 14.55 & 13.85 & 13.59 & 17.25 & 16.13 \\
\hline & $\max$ & 16.89 & 14.16 & 13.71 & 14.81 & 15.49 & 16.13 & 13.91 & 14.87 & 15.76 & 15.98 & 15.36 & 14.85 & 14.29 & 13.92 & 17.57 & 16.39 \\
\hline & $\min$ & 16.08 & 13.47 & 13.05 & 14.24 & 14.86 & 15.46 & 13.30 & 14.16 & 14.95 & 15.34 & 14.73 & 14.20 & 13.50 & 13.30 & 16.90 & 15.84 \\
\hline \multirow{3}{*}{$\begin{array}{c}\% \\
\text { Var }\end{array}$} & Mean & -20.21 & -0.68 & 2.43 & -5.41 & -10.42 & -14.99 & 1.09 & -5.75 & -11.80 & -13.82 & -9.34 & -5.86 & -0.76 & 1.15 & -25.52 & -17.36 \\
\hline & $\max$ & -16.98 & 2.01 & 5.06 & -3.63 & -8.13 & -12.47 & 3.23 & -3.01 & -8.77 & -11.61 & -7.17 & -3.31 & 1.76 & 3.21 & -22.98 & -15.22 \\
\hline & $\min$ & -22.91 & -3.02 & 0.24 & -7.75 & -12.71 & -17.38 & -1.17 & -8.22 & -14.65 & -16.27 & -11.75 & -8.05 & -3.98 & -1.24 & -27.83 & -19.21 \\
\hline \multicolumn{18}{|c|}{ Birds } \\
\hline \multirow[b]{3}{*}{ MSR } & Mean & 6.49 & 6.49 & 7.10 & 7.62 & 8.16 & 7.27 & 7.62 & 7.01 & 7.36 & 7.13 & 7.40 & 7.07 & 7.82 & 6.88 & 6.72 & 7.52 \\
\hline & $\max$ & 6.68 & 6.68 & 7.26 & 7.76 & 8.34 & 7.46 & 7.77 & 7.21 & 7.54 & 7.28 & 7.57 & 7.25 & 7.98 & 7.04 & 6.84 & 7.74 \\
\hline & $\min$ & 6.32 & 6.35 & 6.95 & 7.50 & 7.98 & 7.13 & 7.49 & 6.79 & 7.19 & 6.97 & 7.21 & 6.91 & 7.68 & 6.67 & 6.53 & 7.36 \\
\hline \multirow{3}{*}{$\begin{array}{c}\% \\
\text { Var }\end{array}$} & Mean & 2.71 & 2.72 & -6.41 & -14.21 & -22.37 & -9.06 & -14.22 & -5.12 & -10.47 & -6.87 & -10.95 & -6.07 & -17.36 & -3.27 & -0.75 & -12.85 \\
\hline & $\max$ & 5.28 & 4.75 & -4.29 & -12.46 & -19.74 & -6.91 & -12.34 & -1.85 & -7.91 & -4.52 & -8.14 & -3.63 & -15.23 & -0.07 & 2.11 & -10.40 \\
\hline & $\min$ & -0.13 & -0.22 & -8.92 & -16.36 & -25.14 & -11.87 & -16.49 & -8.10 & -13.06 & -9.18 & -13.58 & -8.72 & -19.68 & -5.60 & -2.58 & -16.02 \\
\hline \multicolumn{18}{|c|}{ Butterflies } \\
\hline \multirow[b]{3}{*}{ MSR } & Mean & 8.76 & 9.34 & 9.31 & 9.74 & 9.72 & 9.37 & 10.17 & 9.55 & 9.46 & 9.44 & 9.83 & 10.00 & 9.65 & 10.18 & 9.33 & 9.60 \\
\hline & $\max$ & 9.02 & 9.59 & 9.52 & 9.92 & 9.87 & 9.64 & 10.38 & 9.80 & 9.69 & 9.66 & 10.07 & 10.22 & 9.82 & 10.44 & 9.56 & 9.84 \\
\hline & $\min$ & 8.48 & 9.15 & 9.11 & 9.53 & 9.54 & 9.17 & 9.94 & 9.21 & 9.29 & 9.23 & 9.62 & 9.79 & 9.48 & 9.94 & 9.16 & 9.37 \\
\hline \multirow{3}{*}{$\begin{array}{c}\% \\
\text { Var }\end{array}$} & Mean & -0.18 & -6.87 & -6.46 & -11.46 & -11.12 & -7.14 & -16.40 & -9.21 & -8.28 & -8.02 & -12.45 & -14.40 & -10.44 & -16.48 & -6.74 & -9.82 \\
\hline & $\max$ & 3.00 & -4.73 & -4.23 & -9.00 & -9.14 & -4.93 & -13.67 & -5.41 & -6.31 & -5.58 & -10.01 & -12.05 & -8.45 & -13.95 & -4.75 & -7.14 \\
\hline & $\min$ & -3.17 & -9.76 & -8.92 & -13.45 & -12.96 & -10.30 & -18.76 & -12.09 & -10.82 & -10.49 & -15.20 & -16.87 & -12.33 & -19.38 & -9.42 & -12.62 \\
\hline
\end{tabular}




\begin{tabular}{|c|c|c|c|c|c|c|c|c|c|c|c|c|c|c|c|c|c|}
\hline \multicolumn{2}{|c|}{$3 \mathrm{~km}$-Landsat } & 1985 & 1987 & 1994 & 1995 & 1996 & 1997 & 1998 & 2001 & 2003 & 2005 & 2006 & 2007 & 2008 & 2009 & 2010 & 2011 \\
\hline \multicolumn{18}{|c|}{ Plants } \\
\hline \multirow[b]{3}{*}{ MSR } & Mean & 243.43 & 259.86 & 214.63 & 234.83 & 250.49 & 238.60 & 252.17 & 269.33 & 232.44 & 245.49 & 256.12 & 229.10 & 237.66 & 224.10 & 248.75 & 248.21 \\
\hline & $\max$ & 248.16 & 265.53 & 220.19 & 239.11 & 255.98 & 243.86 & 258.84 & 276.66 & 237.76 & 251.21 & 261.33 & 233.62 & 242.78 & 229.15 & 253.14 & 254.22 \\
\hline & $\min$ & 236.34 & 254.92 & 209.62 & 229.63 & 244.70 & 234.64 & 245.63 & 263.98 & 226.87 & 239.15 & 250.93 & 223.93 & 232.72 & 218.85 & 242.54 & 243.68 \\
\hline \multirow{3}{*}{$\begin{array}{c}\% \\
\text { Var }\end{array}$} & Mean & -6.53 & -13.73 & 6.07 & -2.77 & -9.62 & -4.42 & -10.36 & -17.87 & -1.73 & -7.44 & -12.09 & -0.26 & -4.01 & 1.92 & -8.86 & -8.62 \\
\hline & $\max$ & -3.43 & -11.56 & 8.26 & -0.50 & -7.09 & -2.69 & -7.50 & -15.53 & 0.71 & -4.66 & -9.82 & 2.00 & -1.85 & 4.22 & -6.14 & -6.64 \\
\hline & $\min$ & -8.61 & -16.20 & 3.64 & -4.65 & -12.03 & -6.72 & -13.28 & -21.08 & -4.05 & -9.94 & -14.37 & -2.24 & -6.25 & -0.28 & -10.78 & -11.26 \\
\hline \multicolumn{18}{|c|}{ Beetles } \\
\hline \multirow[b]{3}{*}{ MSR } & Mean & 14.34 & 14.32 & 13.91 & 13.09 & 13.41 & 13.15 & 13.42 & 13.56 & 14.97 & 12.55 & 16.15 & 13.44 & 13.69 & 15.02 & 16.75 & 14.90 \\
\hline & $\max$ & 14.62 & 14.61 & 14.19 & 13.36 & 13.80 & 13.42 & 13.70 & 13.86 & 15.35 & 12.89 & 16.48 & 13.88 & 13.94 & 15.36 & 17.03 & 15.22 \\
\hline & $\min$ & 14.02 & 13.92 & 13.61 & 12.74 & 13.00 & 12.88 & 13.14 & 13.21 & 14.64 & 12.25 & 15.82 & 13.19 & 13.45 & 14.66 & 16.29 & 14.63 \\
\hline \multirow{3}{*}{$\begin{array}{c}\% \\
\text { Var }\end{array}$} & Mean & -4.33 & -4.17 & -1.19 & 4.75 & 2.47 & 4.32 & 2.37 & 1.35 & -8.88 & 8.69 & -17.47 & 2.19 & 0.43 & -9.28 & -21.85 & -8.43 \\
\hline & $\max$ & -1.97 & -1.25 & 0.96 & 7.31 & 5.38 & 6.30 & 4.41 & 3.90 & -6.52 & 10.84 & -15.07 & 4.06 & 2.15 & -6.65 & -18.51 & -6.44 \\
\hline & $\min$ & -6.37 & -6.32 & -3.25 & 2.83 & -0.42 & 2.33 & 0.36 & -0.83 & -11.66 & 6.20 & -19.87 & -0.96 & -1.40 & -11.72 & -23.93 & -10.72 \\
\hline \multicolumn{18}{|c|}{ Birds } \\
\hline \multirow[b]{3}{*}{ MSR } & Mean & 6.80 & 7.29 & 8.12 & 7.46 & 7.11 & 7.50 & 7.85 & 8.76 & 6.87 & 9.01 & 8.02 & 7.13 & 7.31 & 7.32 & 7.22 & 7.27 \\
\hline & $\max$ & 6.92 & 7.48 & 8.32 & 7.67 & 7.30 & 7.74 & 8.03 & 8.95 & 7.04 & 9.26 & 8.17 & 7.30 & 7.47 & 7.49 & 7.37 & 7.42 \\
\hline & $\min$ & 6.66 & 7.16 & 7.89 & 7.27 & 6.93 & 7.35 & 7.69 & 8.60 & 6.73 & 8.86 & 7.85 & 7.01 & 7.07 & 7.16 & 7.02 & 7.09 \\
\hline \multirow{3}{*}{$\begin{array}{c}\% \\
\text { Var }\end{array}$} & Mean & -2.04 & -9.30 & -21.74 & -11.84 & -6.60 & -12.54 & -17.80 & -31.43 & -3.09 & -35.14 & -20.24 & -6.91 & -9.70 & -9.72 & -8.23 & -9.01 \\
\hline & $\max$ & 0.08 & -7.44 & -18.37 & -8.97 & -3.97 & -10.25 & -15.37 & -28.94 & -0.99 & -32.96 & -17.73 & -5.14 & -6.11 & -7.34 & -5.30 & -6.35 \\
\hline & $\min$ & -3.84 & -12.15 & -24.77 & -15.06 & -9.48 & -16.06 & -20.49 & -34.21 & -5.57 & -38.93 & -22.54 & -9.46 & -12.06 & -12.34 & -10.51 & -11.24 \\
\hline \multicolumn{18}{|c|}{ Butterflies } \\
\hline \multirow[b]{3}{*}{ MSR } & Mean & 9.63 & 10.25 & 8.34 & 7.80 & 10.67 & 9.65 & 9.26 & 10.47 & 9.54 & 10.23 & 10.06 & 9.15 & 9.58 & 10.76 & 10.09 & 10.06 \\
\hline & $\max$ & 9.83 & 10.44 & 8.54 & 8.02 & 10.90 & 9.86 & 9.49 & 10.73 & 9.82 & 10.51 & 10.40 & 9.35 & 9.82 & 11.06 & 10.33 & 10.24 \\
\hline & $\min$ & 9.44 & 10.02 & 8.17 & 7.59 & 10.49 & 9.44 & 9.05 & 10.25 & 9.35 & 10.02 & 9.79 & 8.94 & 9.44 & 10.55 & 9.80 & 9.81 \\
\hline \multirow{3}{*}{$\begin{array}{c}\% \\
\text { Var }\end{array}$} & Mean & -10.21 & -17.30 & 4.58 & 10.73 & -22.11 & -10.42 & -5.91 & -19.74 & -9.11 & -17.08 & -15.06 & -4.66 & -9.57 & -23.05 & -15.40 & -15.08 \\
\hline & $\max$ & -8.03 & -14.61 & 6.53 & 13.22 & -20.06 & -7.97 & -3.49 & -17.28 & -6.97 & -14.68 & -12.02 & -2.32 & -8.04 & -20.67 & -12.16 & -12.26 \\
\hline & $\min$ & -12.43 & -19.46 & 2.29 & 8.26 & -24.72 & -12.85 & -8.52 & -22.76 & -12.40 & -20.24 & -19.00 & -6.94 & -12.39 & -26.58 & -18.19 & -17.17 \\
\hline
\end{tabular}


Appendix V. Example of the output of stepwise regression models.

MODIS (2000)-1 × 1 km-Beetles

Fina1 Mode1 Output

\begin{tabular}{|c|c|c|c|}
\hline \multicolumn{4}{|c|}{ Model Summary } \\
\hline $\mathrm{R}$ & 0.210 & RMSE & 3.665 \\
\hline R-Squared & 0.044 & Coef. Var & 35.245 \\
\hline Adj. R-Squared & 0.033 & MSE & 13.430 \\
\hline Pred R-Squared & 0.007 & MAE & 2.872 \\
\hline
\end{tabular}

RMSE: Root Mean Square Error

MSE: Mean Square Error

MAE: Mean Absolute Error

ANOVA

\begin{tabular}{|c|c|c|c|c|c|c|}
\hline & $\begin{array}{l}\text { Sum of } \\
\text { Squares }\end{array}$ & DF & Mean & square & $\mathrm{F}$ & sig. \\
\hline $\begin{array}{l}\text { Regression } \\
\text { Residual } \\
\text { Total }\end{array}$ & $\begin{array}{r}56.141 \\
1222.139 \\
1278.280\end{array}$ & $\begin{array}{r}1 \\
91 \\
92\end{array}$ & & $\begin{array}{l}56.141 \\
13.430\end{array}$ & 4.18 & 0.0438 \\
\hline
\end{tabular}

Parameter Estimates

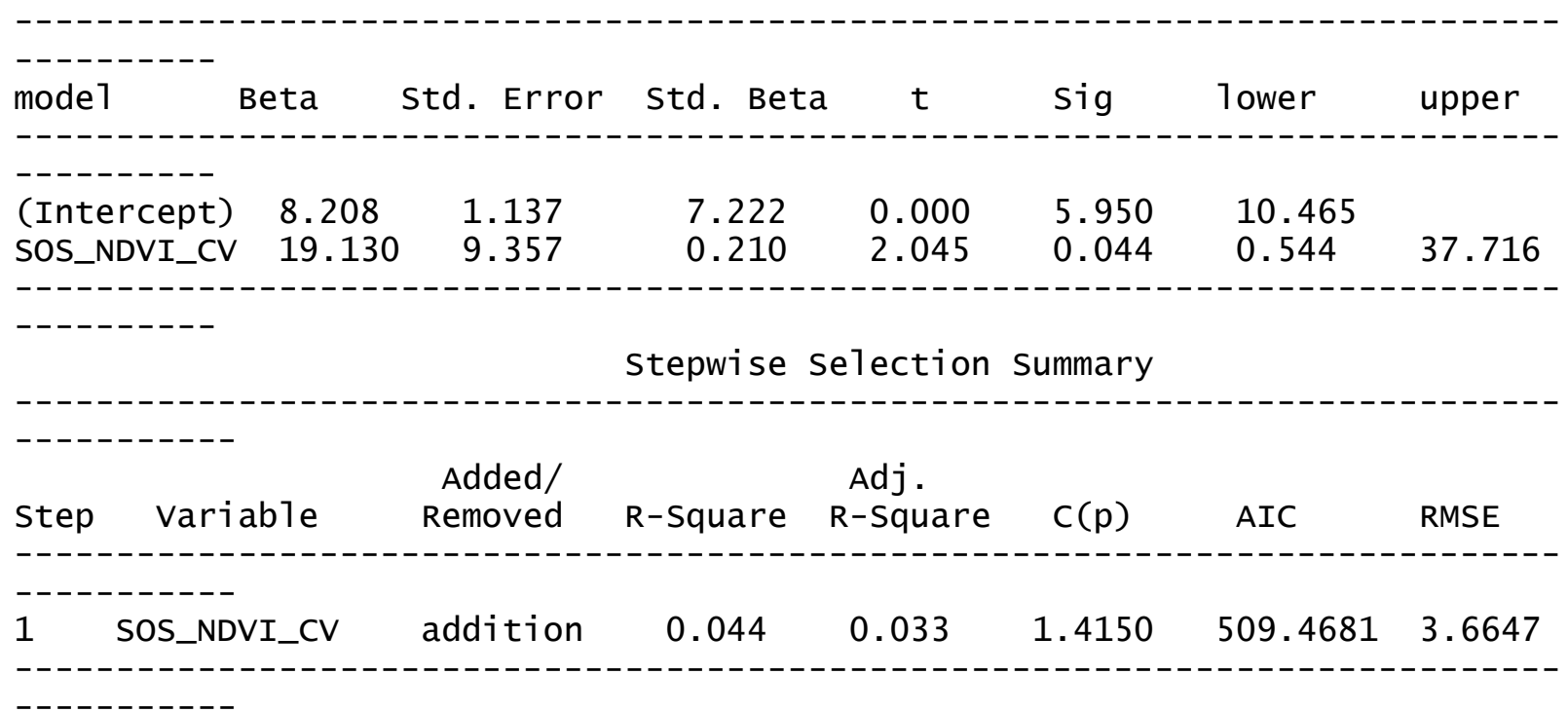

